\title{
THE ROLE OF FOLATE IN NON-ALCOHOLIC FATTY LIVER DISEASE
}

By Victoria Sid

A thesis submitted to the Faculty of Graduate Studies of the University of Manitoba in partial fulfillment of the requirement of the degree of

DOCTOR OF PHILOSOPHY

Department of Physiology and Pathophysiology

Rady Faculty of Health Sciences

University of Manitoba

Winnipeg, Manitoba, Canada

Copyright @ 2018 by Victoria Sid 


\section{ABSTRACT}

Non-alcoholic fatty liver disease (NAFLD) is a spectrum of chronic liver diseases that are characterized by steatosis (hepatic lipid accumulation), inflammation, fibrosis, and liver injury. Chronic consumption of high-fat diets is a major cause of obesity and NAFLD. Because NAFLD is a multifaceted disorder with many underlying metabolic abnormalities, currently, there is no pharmacological agent that is therapeutically approved for the treatment of NAFLD. Folate is a water-soluble B vitamin that has been demonstrated to have antioxidant functions and lipidlowering effects. The general objective of my research was to investigate the role of folate in the context of high-fat diet-induced NAFLD.

A low level of folate in the circulation is often observed in patients with obesity. A low level of endogenous folates in rodents perturbs folate-dependent one-carbon metabolism, and may be associated with development of metabolic diseases such as NAFLD. The first part of my research focused on identifying the underlying mechanisms that are responsible for regulating folate status during high-fat diet feeding. Our results have shown that high-fat diet feeding reduces folate levels in the liver and serum by attenuating expression of hepatic folate transporters in mice. Such effect is mediated through inhibition of nuclear respiratory factor-1 (NRF-1), an important transcriptional regulator of folate transporters.

Hepatic lipid and glucose metabolism is frequently perturbed in patients with NAFLD. Similarly, high-fat diet feeding was demonstrated to significantly elevate lipid and glucose levels in the liver of rodents. The second part of my research aimed to investigate the effects of folic acid supplementation on hepatic metabolic regulation in high-fat diet fed mice. Our results have demonstrated that folic acid supplementation effectively lowers hepatic cholesterol and glucose levels in high-fat diet fed mice through activation of AMPK, a master regulator of whole body 
energy balance and metabolic homeostasis. Activation of AMPK by folic acid was mediated through an elevation of its allosteric activator AMP and phosphorylation of its upstream kinase, namely, liver kinase B1 (LKB1) in the liver.

Hepatic inflammation is an important mediator of NAFLD pathogenesis. The third part of my research aimed to investigate the regulation of hepatic inflammation by folic acid supplementation in high-fat diet fed mice. Our results have shown that folic acid supplementation attenuated the inflammatory response induced by high-fat diet consumption by reducing the expression of inflammatory cytokines (IL-6, TNF- $\alpha$ ) in mouse liver. The antiinflammatory effect of folic acid was mediated via inhibition of NF- $\mathrm{BB}$, a key transcriptional regulator of pro-inflammatory genes. This was associated with decreased deposition of hepatic lipids and inflammatory foci in mice.

Taken together, our results have demonstrated that chronic consumption of high-fat diets leads to development of metabolic abnormalities and disrupts folate homeostasis, which results in low folate status in the body. Folic acid supplementation can restore hepatic metabolism and reduce inflammation in high-fat diet fed mice. This suggests that folic acid may have therapeutic implications for the management of NAFLD. 


\section{ACKNOWLEDGMENTS}

First and foremost, I would like to thank my supervisor, Dr. Karmin O, for giving me the opportunity to pursue graduate studies in her laboratory. She has not only been an incredible mentor, but also an inspiring role model to learn from. I admire her hard work and dedication to research, as well as her patience and willingness to demonstrate in the lab. Everything I learned from her has shaped me as a better researcher and helped me grow as a person. I will always be grateful for all the support she has given me during my time as a graduate student.

I would also like to thank the members of my advisory committee, Dr. Grant Pierce, Dr. James House and Dr. Chris Siow, for sharing their expertise and research advice during committee meetings. They have greatly challenged me on an intellectual level, which helped me to improve my knowledge and research. I really appreciate their time and support through the past few years.

I would like to acknowledge my past and present lab mates Sun-young Hwang, Lindsei Sarna, Nan Wu, Pengqi Wang, Caitlin Sarna, Sirini Amarakoon, Yvette Shang and Susara Madduma Hewage, for their encouragement and assistance. I would like to extend special thanks to Nan Wu and Lindsei Sarna, for their kind mentorship during my early years of research training.

I would like to thank my parents, Andy and Hing Sid, as well as my beloved grandmother and sister, Linda Sid. Pursuing graduate studies would have not been possible without their unconditional love and support.

Lastly, I would like to acknowledge the research studentship from The Natural Sciences and Engineering Research Council (NSERC) and St. Boniface Hospital Research Foundation during the course of my graduate studies. 


\section{PUBLICATIONS}

\section{Research articles:}

1. Sid V, Siow YL, Shang Y, Woo CW, O K. High-fat diet consumption reduces hepatic folate transporter expression via nuclear respiratory factor-1. J Mol Med (Berl). 2018; 96(11):12031213.

2. Sid V, Shang Y, Siow YL, Madduma Hewage S, House JD, O K. Folic acid supplementation attenuates chronic hepatic inflammation in high-fat diet fed mice. Lipids. 2018; 53 (7):709716.

3. Sid V, Siow, YL, O K. Role of folate in non-alcoholic fatty liver disease (NAFLD). Can J Physiol Pharmacol. 2018; 95(10):1141-1148.

4. Sarna LK, Sid V, Wang P, Siow YL, House JD, O K. Tyrosol attenuates high-fat dietinduced hepatic oxidative stress: potential involvement of cystathionine beta-synthase and cystathionine gamma-lyase. Lipids. 2016; 51(5):583-90.

5. Sid V, Wu N, Sarna LK, Siow YL, House JD, O K. Folic acid supplementation during highfat diet feeding restores AMPK activation via an AMP-LKB1-dependent mechanism. Am J Physiol Regul Integr Comp Physiol. 2015; 309(10):R1215-25.

\section{Abstracts published:}

1. Sid V, Shang Y, Madduma Hewage S, Siow YL, O K. Folic Acid Improves Lipid Metabolism and Reduces Hepatic Inflammation in Diet-induced Obesity and Non-alcoholic Fatty Liver Disease. Atherosclerosis Supplements. 2018; 32:136.

2. Madduma Hewage S, Sid V, Prashar S, Shang Y, O K, Siow YL. Impact of Lingonberry Supplementation on High-fat Diet-induced Metabolic Syndrome and Hyperlipidemia. Atherosclerosis Supplements. 2018; 32:65.

3. Sid V, Shang Y, Siow YL, O K. Dietary regulation of inflammatory response in nonalcoholic fatty liver disease (NAFLD). Canadian Journal of Cardiology. 2017; 33(10):S2728.

4. Sid V, Shang Y, Siow YL, O K. Regulation of Hepatic Inflammation by Folic Acid in Nonalcoholic Fatty Liver Disease (NAFLD). FASEB J. 2017; 30:780.8.

5. Sid V, Wu N, Sarna LK, Shang Y, Siow YL, House JD, O K. Regulation of AMPK and hepatic metabolism by folic acid supplementation in non-alcoholic fatty liver disease. FASEB J. 2016; 30:870.3.

6. Sid V, Wu N, Sarna LK, Siow YL, O K. Regulation of AMP-activated protein kinase and cholesterol biosynthesis in non-alcoholic fatty liver disease. Canadian Journal of Cardiology. 2015; 31:S232-233

7. Sid V, Shang Y, Sarna LK, Wang P, Siow YL, O K. Supplementation of Folic Acid Attenuates Hepatic Inflammation in High Fat Diet Fed Mice. FASEB J. 2015; 29:884.45. 


\section{TABLE OF CONTENTS}

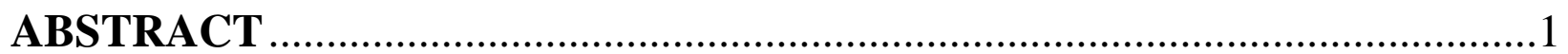

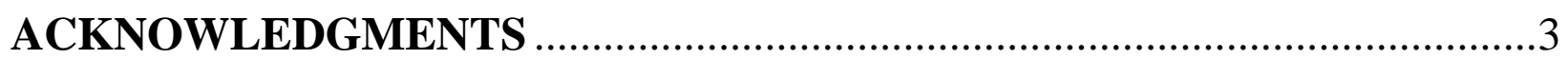

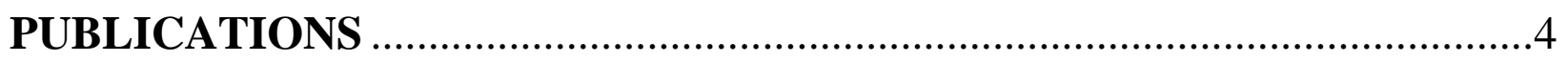

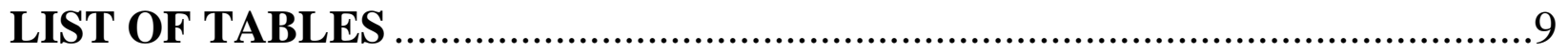

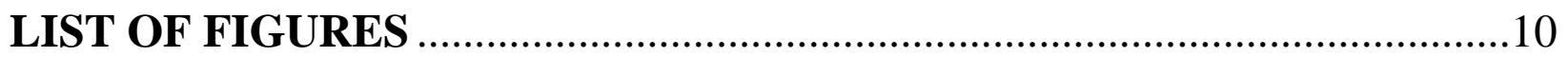

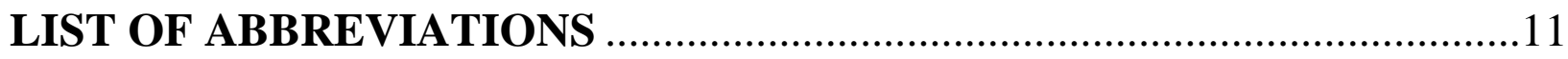

LIST OF PERMISSIONS FOR COPYRIGHTED MATERIAL .....................14

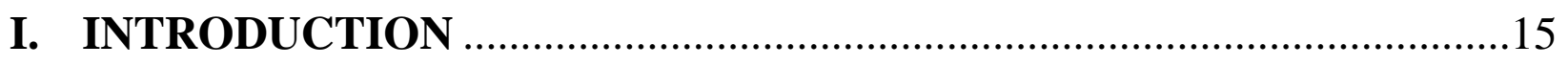

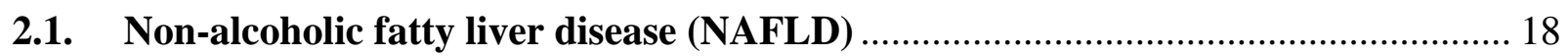

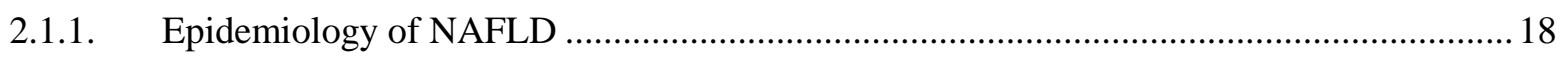

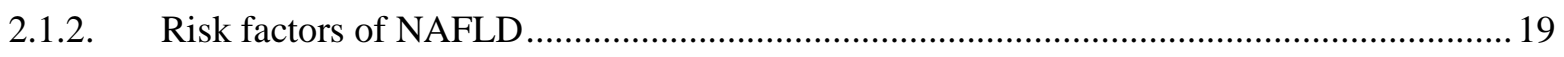

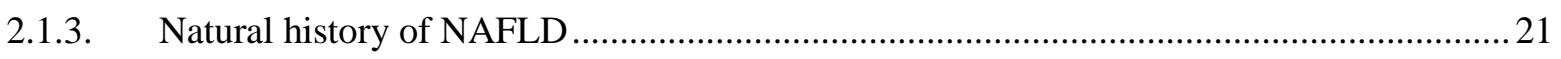

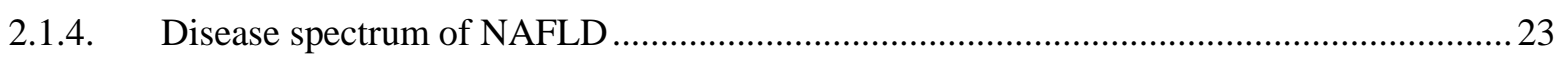

2.1.5. Pathogenesis of NAFLD ...................................................................................... 26

2.1.6. Animal and cell models for studying NAFLD ...............................................................2

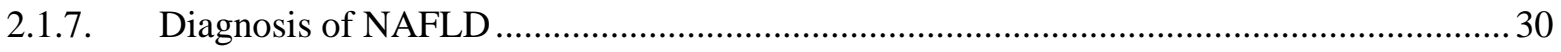

2.1.8. NAFLD management ............................................................................................ 31

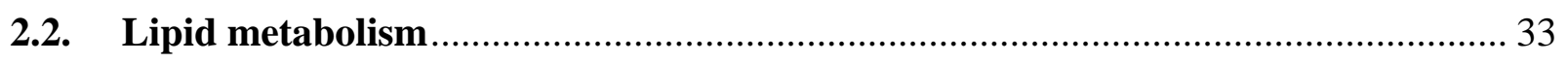

2.2.1. Absorption of dietary lipids and delivery to peripheral tissues....................................... 33

2.2.2. Triglyceride and cholesterol metabolism .................................................................... 33

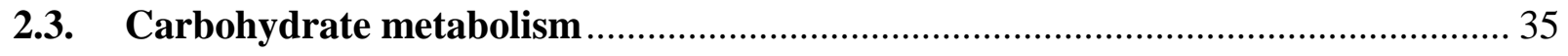

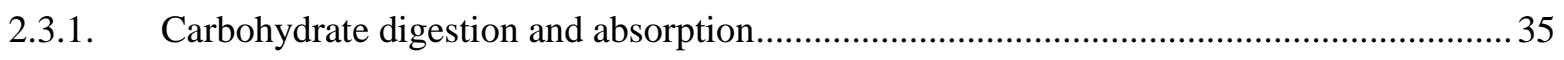

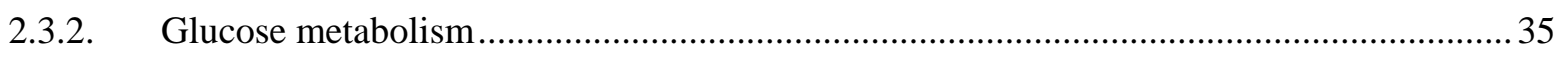

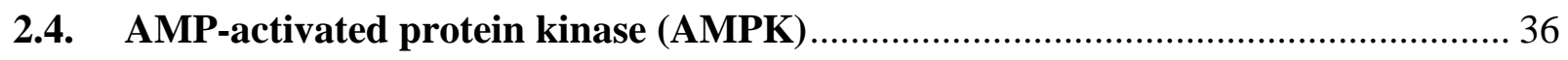

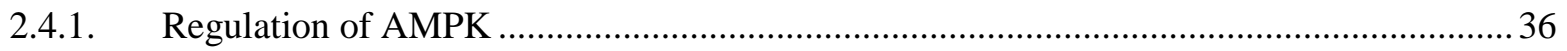

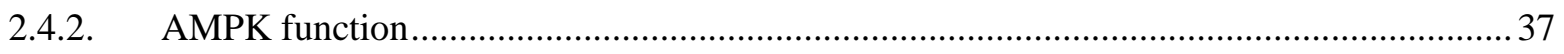

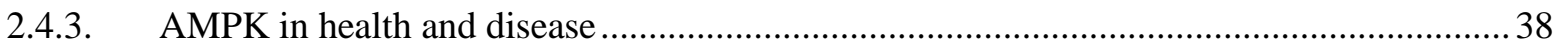

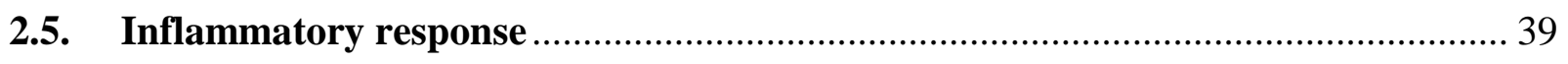

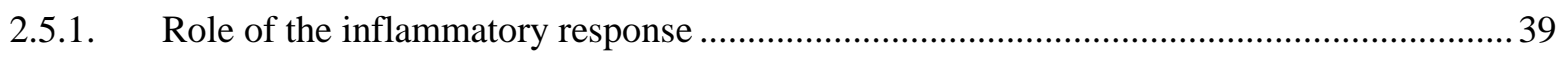




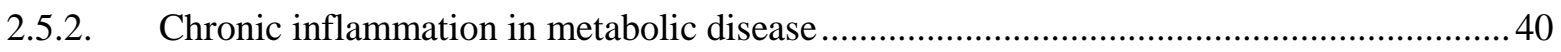

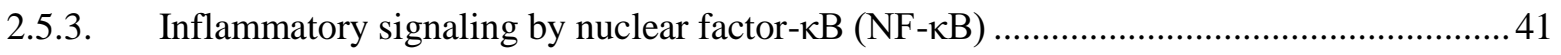

2.6. Role of vitamins in metabolic disease / NAFLD ................................................. 42

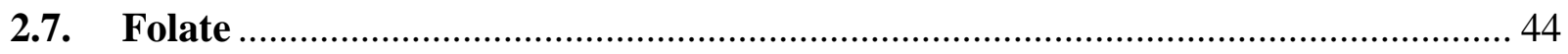

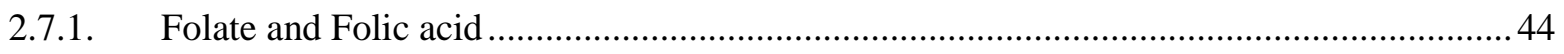

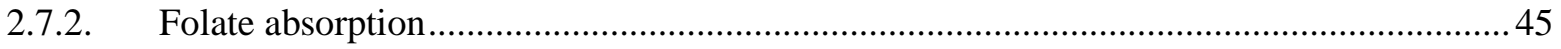

2.7.3. Folate metabolism and biological function ................................................................... 48

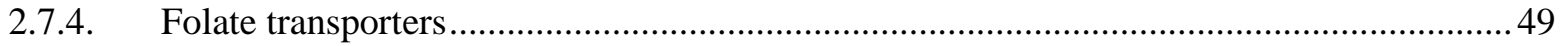

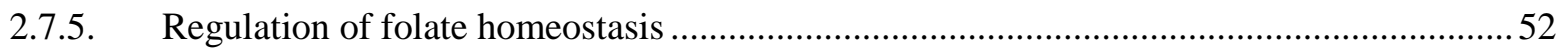

2.7.6. Implication of folate deficiency in disease .................................................................53

2.7.7. Role of folate in lipid and carbohydrate metabolism ....................................................5

2.7.8. Role of folate in oxidative stress and inflammation........................................................55

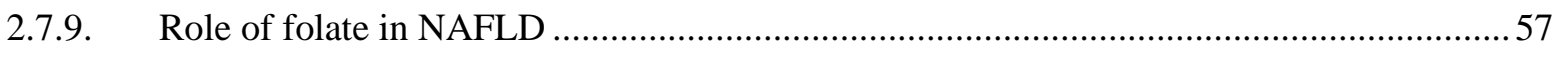

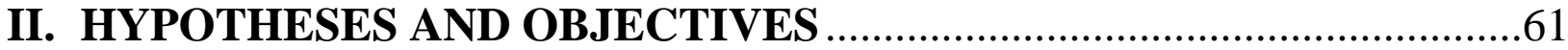

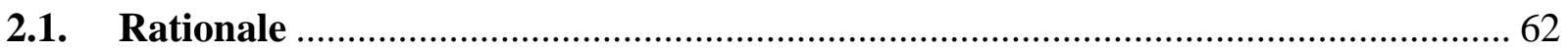

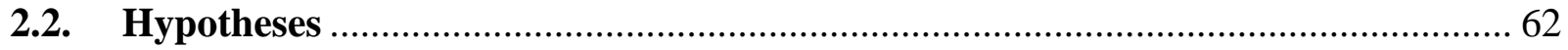

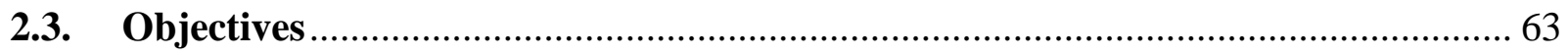

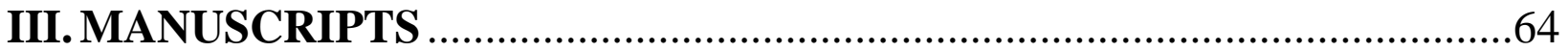

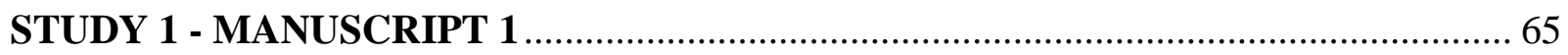

3.1. High-fat diet consumption reduces hepatic folate transporter expression via

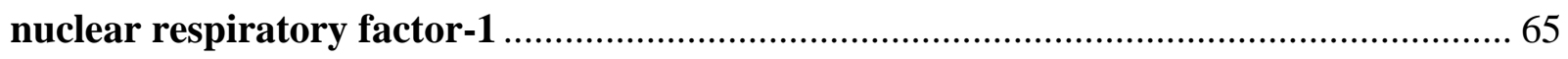

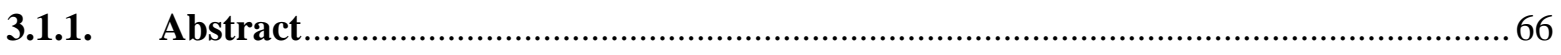

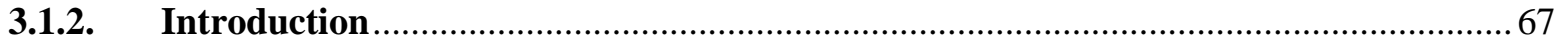

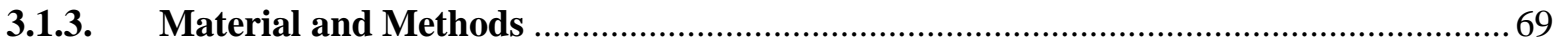

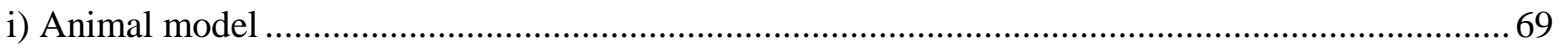

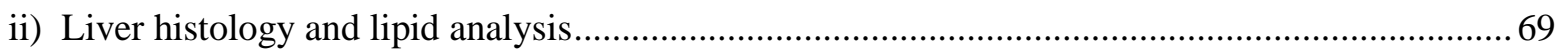

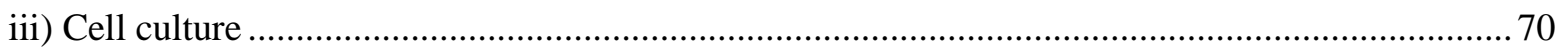

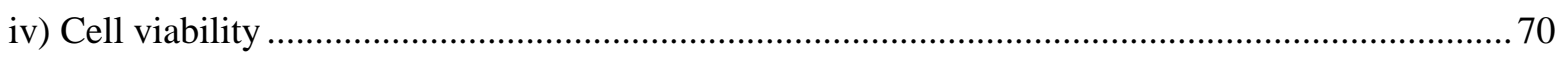

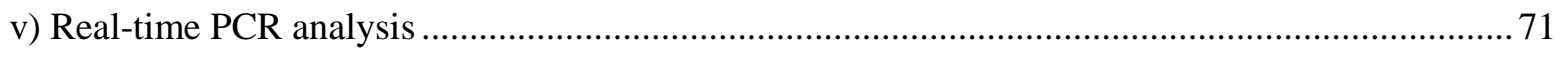

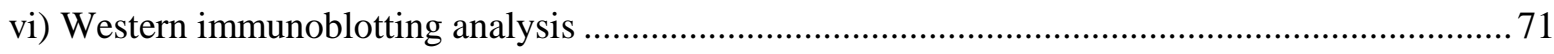

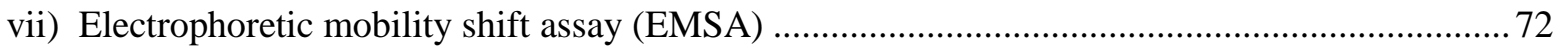

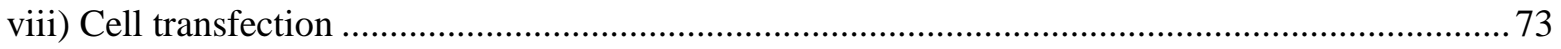




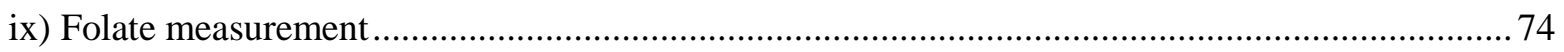

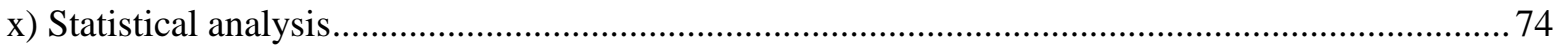

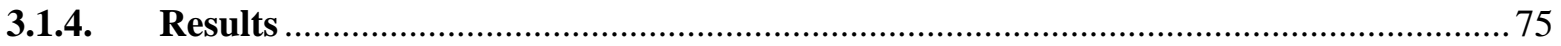

i) High-fat diet feeding reduced folate levels in the liver and serum ............................................. 75

ii) Decreased folate transporter expression in the liver of mice fed a high-fat diet and in fatty acid

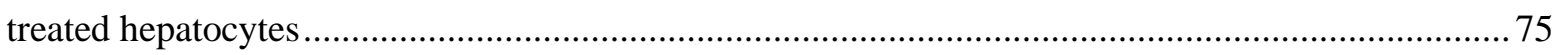

iii) Reduction of hepatic folate transporter expression was mediated by NRF-1 …........................ 76

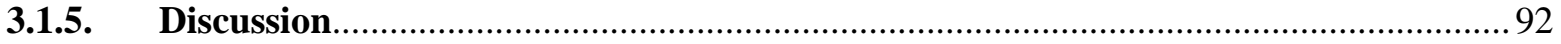

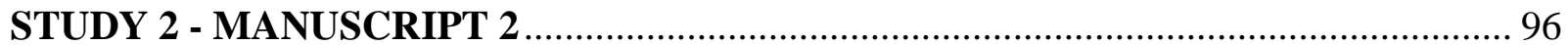

3.2. Folic acid supplementation during high-fat diet feeding restores AMP-activated protein kinase (AMPK) activation via an AMP-LKB1 dependent mechanism .............. 96

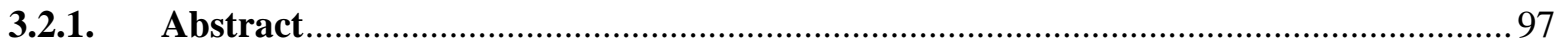

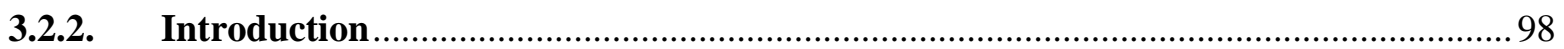

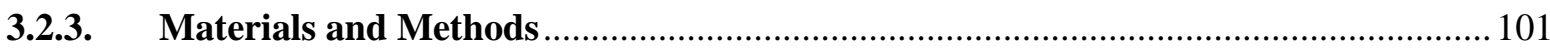

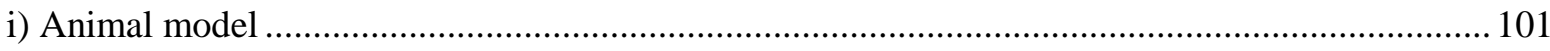

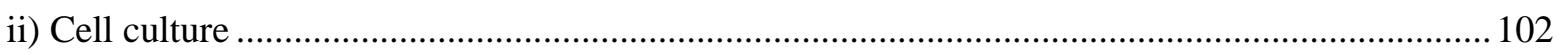

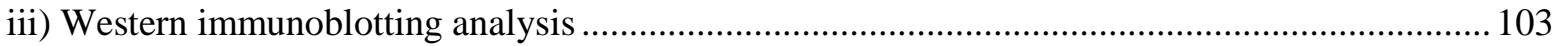

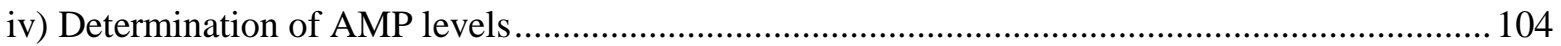

v) Measurement of cholesterol and HMG-CoA reductase activity ................................................ 104

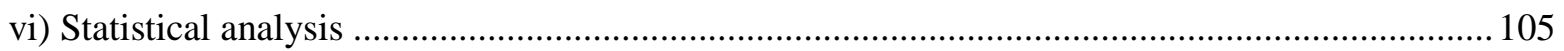

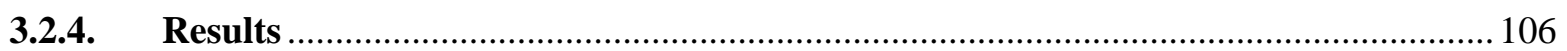

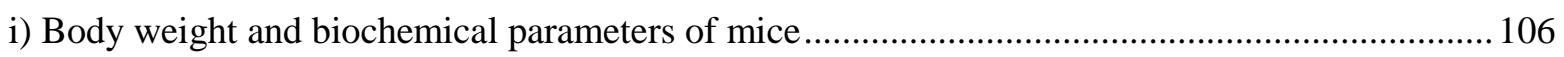

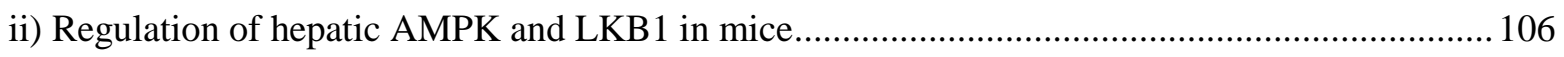

iii) Effect of fatty acid and folic acid on AMPK and LKB1 activation in hepatocytes .................... 107

iv) Role of AMP on AMPK activation in hepatocytes and liver tissue ......................................... 108

v) Effect of folic acid supplementation on hepatic cholesterol production..................................... 109

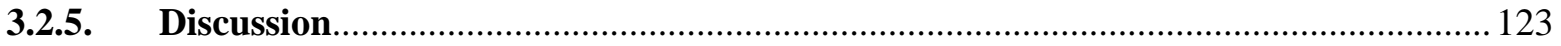

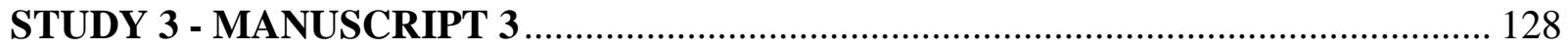

3.3. Folic acid supplementation attenuates chronic hepatic inflammation in high-fat

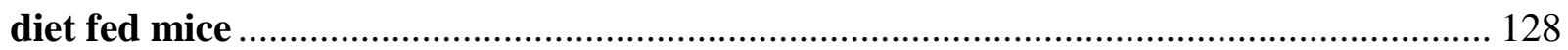

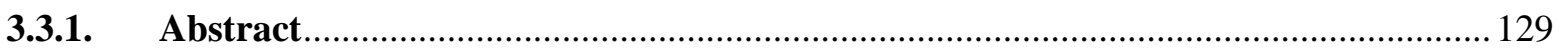

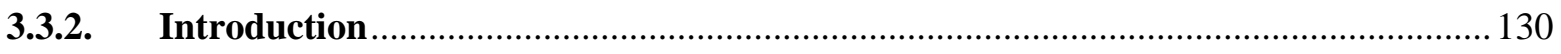

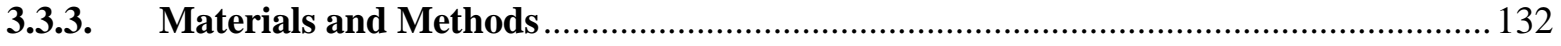




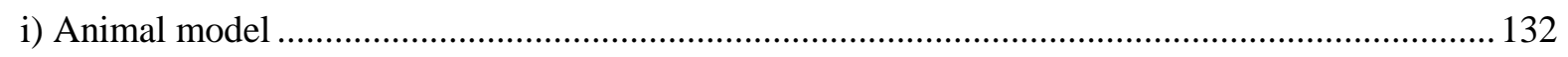

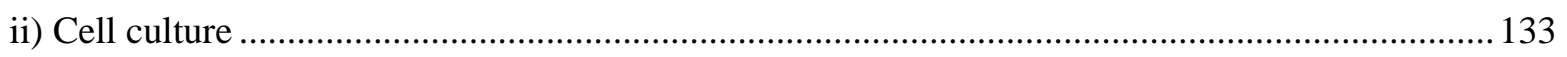

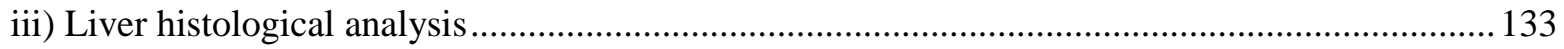

iv) Real-time polymerase chain reaction (PCR) analysis ........................................................... 134

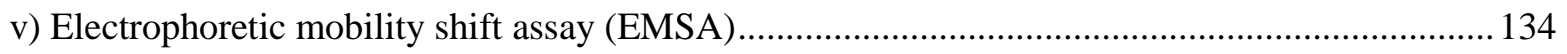

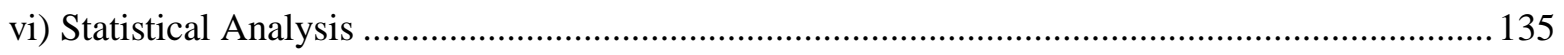

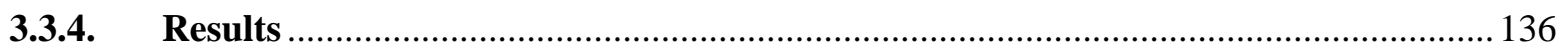

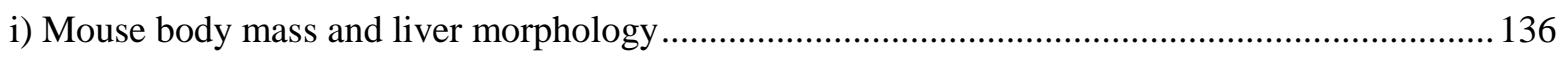

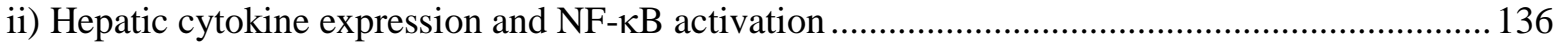

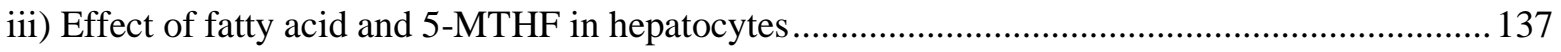

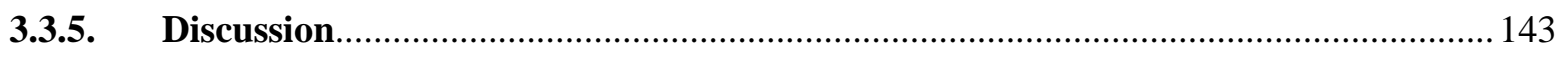

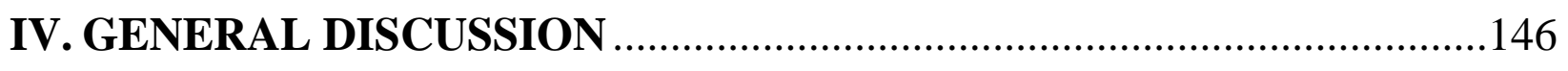

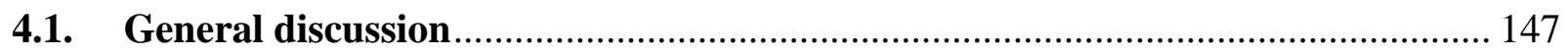

i. Study 1- High-fat diet consumption reduces hepatic folate transporter expression via

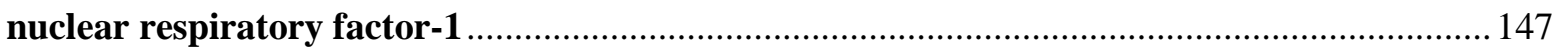

ii. Study 2- Folic acid supplementation during high-fat diet feeding restores AMPK activation via an AMP-LKB1-dependent mechanism ........................................................ 149

iii. Study 3- Folic acid supplementation attenuates chronic hepatic inflammation in high-

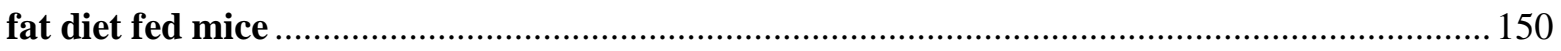

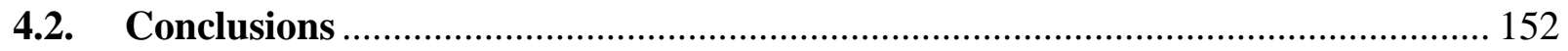

V. FUTURE PERSPECTIVES ...................................................... 153

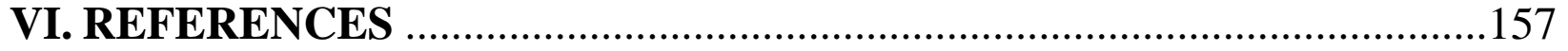

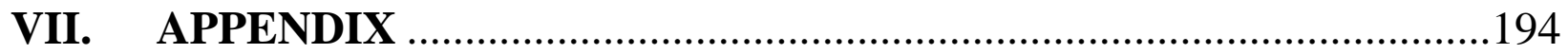

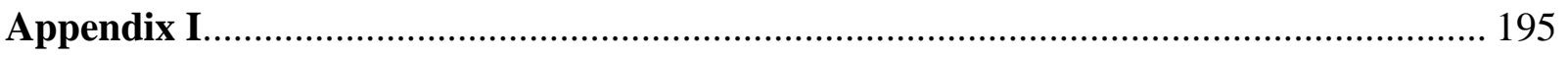

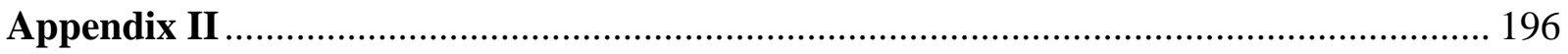

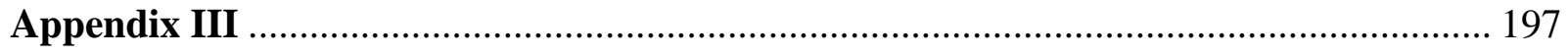




\section{LIST OF TABLES}

Table 1. Primer sequences of folate transporters and other folate metabolism genes..............................91

Table 2. Composition of purified diets: energy, macronutrient and folic acid content........................... 110

Table 3. Composition of purified diets: energy, macronutrient and other primary ingredients ................ 138 


\section{LIST OF FIGURES}

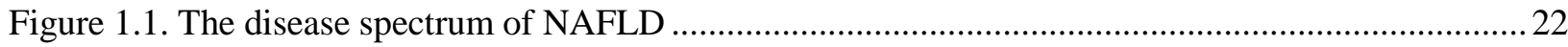

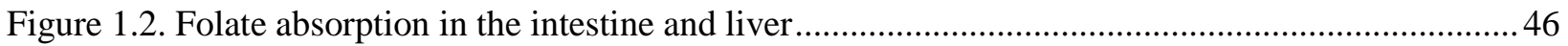

Figure 1.3. Compartmentalization of folate-dependent one-carbon metabolism ......................................50

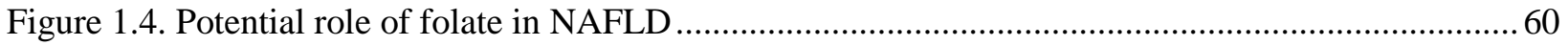

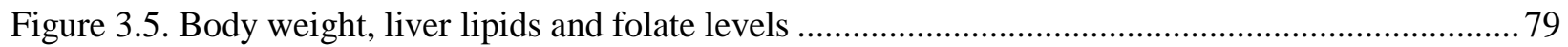

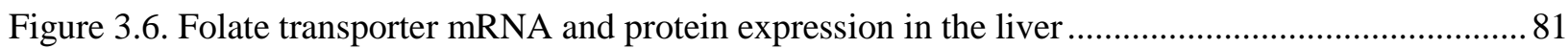

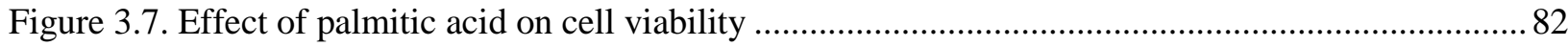

Figure 3.8. Folate transporter expression and folate concentration in HepG2 cells .............................. 84

Figure 3.9. Effect of high-fat diet on NRF-1/DNA binding activity in the liver ......................................8 86

Figure 3.10. Effect of NRF-1 siRNA transfection on folate transporter expression and folate concentration

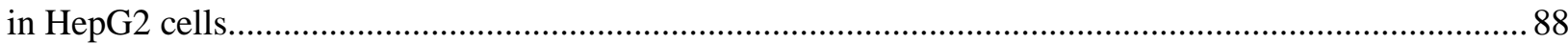

Figure 3.11. Effect of NRF-1 siRNA transfection on cell viability ....................................................89

Figure 3.12. Effect of palmitic acid on folate transporter expression in NRF-1 transfected cells ..............90

Figure 3.13. Body weight, blood glucose and liver lipids .............................................................. 112

Figure 3.14 AMPK and LKB1 phosphorylation in mouse liver ....................................................... 114

Figure 3.15. AMPK and LKB1 phosphorylation in HepG2 cells ..................................................... 118

Figure 3.16. Regulation of LKB1 by folic acid in HepG2 cells .......................................................... 119

Figure 3.17. AMPK phosphorylation in HepG2 cells and AMP levels in mouse liver .......................... 120

Figure 3.18. Determination of HMG-CoA reductase protein and enzyme activity in mouse liver .......... 121

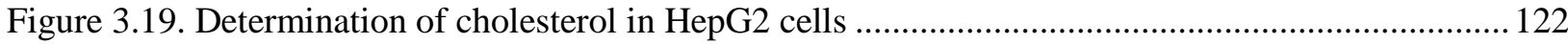

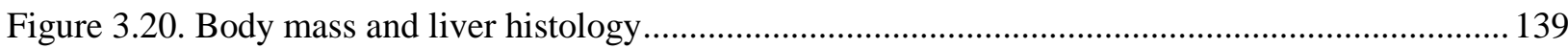

Figure 3.21. Effect of folic acid on inflammatory cytokine expression and NF- $\kappa B$ binding activity in

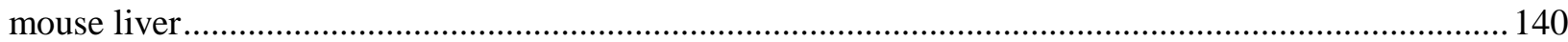

Figure 3.22. Effect of 5-MTHF on inflammatory cytokine expression and NF-kB binding activity in

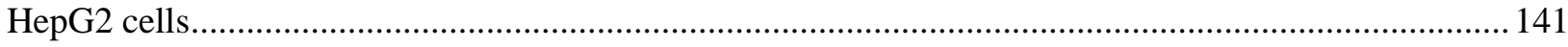

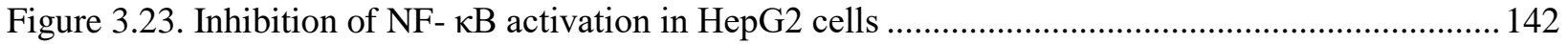




\section{LIST OF ABBREVIATIONS}

\begin{tabular}{|c|c|}
\hline $\mathrm{ACC}$ & acetyl-CoA carboxylase \\
\hline AP-1 & activator protein-1 \\
\hline ACAT & acyl-coenzyme A: cholesterol acyltransferase \\
\hline ALT & alanine aminotransferase \\
\hline AML-12 & alpha mouse liver-12 \\
\hline AICAR & 5-aminoimidazole-4-carboxamide ribonucleotide \\
\hline PDTC & ammonium pyrrolidinedithiocarbamate \\
\hline AMPK & AMP-activated protein kinase \\
\hline ApoC3 & apolipoprotein $\mathrm{C} 3$ \\
\hline ABCG2 & ATP binding cassette subfamily $\mathrm{G}$ member 2 \\
\hline BHMT & betaine hydroxymethyltransferase \\
\hline BMI & body mass index \\
\hline BSA & bovine serum albumin \\
\hline ChREBP & carbohydrate responsive element binding protein \\
\hline CaMKK2 & calcium/calmodulin-dependent protein kinase kinase 2 \\
\hline CT & computed tomography \\
\hline CBS & cystathionine- $\beta$-synthase \\
\hline CSE & cystathionine- $\gamma$-lyase \\
\hline dTMP & deoxythymidylate \\
\hline dUMP & deoxyuridylate \\
\hline DFEs & dietary folate equivalents \\
\hline MTT & 4,5-dimethylthiazol-2-Y1)-2,5-diphenyltetrazolium bromide \\
\hline DGAT & diglyceride acyltransferase \\
\hline $\mathrm{DHF}$ & dihydrofolate \\
\hline DHFR & dihydrofolate reductase \\
\hline DMEM & dulbecco modified eagle medium \\
\hline EMSA & electrophoretic mobility shift assay \\
\hline FAS & fatty acid synthase \\
\hline FATPs & fatty acid transport proteins \\
\hline FBS & fetal bovine serum \\
\hline Folr 1 or FR $\alpha$ & folate receptor alpha \\
\hline FR $\beta$ & folate receptor beta \\
\hline fmet-tRNA & formylmethionine-tRNA \\
\hline 10-formyl-THF & 10-formyl-tetrahydrofolate \\
\hline FTHFS & formyltetrahydrofolate synthetase \\
\hline FT & formyltransferase \\
\hline G-6-Pase & glucose-6-phosphatase \\
\hline GLUT 2 & glucose transporter type 2 \\
\hline GLUT 4 & glucose transporter type 4 \\
\hline $\mathrm{H} \& \mathrm{E}$ & hematoxylin and eosin \\
\hline $\mathrm{HCC}$ & hepatocellular carcinoma \\
\hline HDL & high-density lipoprotein \\
\hline HFD & high-fat diet \\
\hline HMG-CoA & 3-hydroxy-3-methylglutaryl coenzyme A \\
\hline
\end{tabular}




\begin{tabular}{|c|c|}
\hline IKK & inhibitor- $\kappa \mathrm{B}$ kinase \\
\hline $\mathrm{I} \kappa \mathrm{B}$ & inhibitor- $\mathrm{kB}$ \\
\hline IRFs & interferon regulatory factors \\
\hline IL-1 $\beta$ & interleukin-1 $\beta$ \\
\hline IL-6 & interleukin-6 \\
\hline KLF4 & kruppel-like factor 4 \\
\hline LKB1 & liver kinase B1 \\
\hline LDL & low-density lipoprotein \\
\hline MRI & magnetic resonance imaging \\
\hline MAT & methionine adenosyltransferase \\
\hline MCD & methionine-choline deficient \\
\hline MS & methionine synthase \\
\hline 5-MTHF & 5-methyltetrahydrofolate \\
\hline 5,10-methylene-THF & 5,10-methylenetetrahydrofolate \\
\hline MTHFC & methylenetetrahydrofolate cyclohydrolase \\
\hline MTHFD & methylenetetrahydrofolate dehydrogenase \\
\hline MRP3 & multidrug resistance-associated protein 3 \\
\hline MCP-1 & monocyte chemoattractant protein-1 \\
\hline NM & nicotinamide \\
\hline H89 & $\begin{array}{l}\mathrm{N} \text {-[2-(p-bromocinnamylamino)ethyl]-5-isoquinolinesulfonamide } \\
\text { dihydrochloride }\end{array}$ \\
\hline DMG & $N, N$-dimethylglycine \\
\hline NAFLD & non-alcoholic fatty liver disease \\
\hline NASH & non-alcoholic steatohepatitis \\
\hline NCD & normal control diet \\
\hline$N F-\kappa B$ & nuclear factor-kappa B \\
\hline NRF-1 & nuclear respiratory factor- 1 \\
\hline ANOVA & one-way analysis of variance \\
\hline PA or PAM & palmitic acid \\
\hline PNPLA3 & patatin-like phospholipase domain-containing 3 \\
\hline PKA & protein kinase $\mathrm{A}$ \\
\hline $\mathrm{PKC}$ & protein kinase $\mathrm{C}$ \\
\hline $\mathrm{PKC}-\zeta$ & protein kinase $\mathrm{C}$-zeta \\
\hline PEPCK & phosphoenolpyruvate carboxykinase \\
\hline $\mathrm{PC}$ & phosphatidylcholine \\
\hline PE & phosphatidylethanolamine \\
\hline PEMT & phosphatidylethanolamine methyltransferase \\
\hline PCR & polymerase chain reaction \\
\hline PP2A & protein phosphatase $2 \mathrm{~A}$ \\
\hline PP2C & protein phosphatase $2 \mathrm{C}$ \\
\hline PCFT & proton-coupled folate transporter \\
\hline ROS & reactive oxygen species \\
\hline RDA & recommended daily allowance \\
\hline $\mathrm{RFC}$ & reduced folate carrier \\
\hline RLU & relative luminescence units \\
\hline scrRNA & scrambled RNA \\
\hline
\end{tabular}


SHMT

siRNA

$\mathrm{Sp}$

SAM

SAH

SAHH

SREBP-1c

THF

TS

TLR4

TBST

TAK1

TNF- $\alpha$

5'UTR

USF

VLDL serine hydroxymethyltransferase

small interfering RNA

specificity protein

$s$-adenosylmethionine

$s$-adenosylhomocysteine

$s$-adenosylhomocysteine hydrolase

sterol regulatory element binding protein-1c

tetrahydrofolate

thymidylate synthase

toll-like receptor 4

tris-buffered saline with tween-20

transforming growth factor-beta-activating kinase

tumor necrosis factor-alpha

5' untranslated region

upstream stimulatory factor

very low-density lipoprotein 


\section{LIST OF PERMISSIONS FOR COPYRIGHTED MATERIAL}

Role of folate in nonalcoholic fatty liver disease

Sid V, Siow YL, O K.

Can J Physiol Pharmacol. 2017 Oct; 95(10):1141-1148

Copyright (C) 2017 Her Majesty the Queen in right of Canada, author retains copyright

Folic acid supplementation during high-fat diet feeding restores AMPK activation via an AMP-LKB1-dependent mechanism

Sid V, Wu N, Sarna LK, Siow YL, House JD, O K.

Am J Physiol Regul Integr Comp Physiol. 2015 Nov; 309(10):R1215-25

Copyright (C) 2015 the American Physiological Society

High-fat diet consumption reduces hepatic folate transporter expression via nuclear respiratory factor-1

Sid V, Siow YL, Shang Y, Woo CW, O K.

J Mol Med. 2018 Aug; 96(11):1203-1213

Copyright (C) Springer-Verlag GmbH Germany, part of Springer Nature 2018

Folic acid supplementation attenuates chronic hepatic inflammation in high-fat diet fed mice

Sid V, Shang Y, Siow YL, Madduma Hewage S, House JD, O K.

Lipids. 2018 Aug; 53 (7):709-716

Copyright (C) 2018 AOCS

Figure 1.1. The disease spectrum of NAFLD

Image from "Human Fatty Liver Disease: Old Questions and New Insights"

Cohen JC, Horton JD, Hobbs HH.

Science. 2011; 332(6037): 1519-1523.

Copyright (C) 2011 The American Association for the Advancement of Science 


\section{INTRODUCTION}


Non-alcoholic fatty liver disease (NAFLD) represents a broad spectrum of liver diseases that develop independently of excessive alcohol consumption. It ranges from steatosis (hepatic lipid accumulation) to advanced forms such as non-alcoholic steatohepatitis (NASH) and cirrhosis. Cirrhosis can ultimately advance to hepatocellular carcinoma (HCC) (also known as liver cancer) (Cohen et al. 2011). Patients with NAFLD are likely to develop obesity and its associated metabolic comorbidities such as hyperlipidemia, hyperglycemia, insulin resistance, and hypertension (Marchesini et al. 2001, Farrell et al. 2006). It is currently estimated that 83.1 million people in the world suffer from NAFLD (Estes et al. 2018). As the incidence of obesity and type 2 diabetes is expected to rise, NAFLD is becoming more of a health concern (Loomba et al. 2013). The pathophysiology of NAFLD is complex and incompletely understood; therefore, a number of genetic and dietary animal models have been developed to investigate NAFLD pathogenesis (Larter et al. 2008, Takahashi et al. 2012). Because NAFLD is a multifaceted disorder with many underlying metabolic abnormalities, currently, there is no pharmacological agent that is therapeutically approved for the treatment of this disease (Sid et al. 2017). Lifestyle modifications such as exercise and dietary restriction lead to weight loss, which is regarded as the most safe and effective strategy for improving NAFLD. Although weight loss is beneficial for patients with NAFLD, it is difficult to achieve and sustain (Chalasani et al. 2012, Ahmed 2015). Novel treatments strategies are urgently required for NAFLD management. Nutritional therapies are emerging as a promising route for prevention and treatment of metabolic disease (Veena et al. 2014).

Folate refers to a group of water-soluble B9 vitamins that serve as co-enzymatic substrates for one-carbon transfer reactions. Folate-dependent one-carbon metabolism is essential for nucleic acid biosynthesis, methylation reactions, and sulfur-containing amino acid metabolism (Tibbetts 
et al. 2010, Stover et al. 2011). Mammals lack the enzymatic capacity to synthesize folates; therefore, the intake of dietary folates is essential to meet their physiological requirements (Lucock 2000, Zhao et al. 2009). Dietary folates are often in the reduced and polyglutamated forms (Wright et al. 2007, Zhao et al. 2009). In contrast, folic acid is an oxidized monoglutamate with higher bioavailability than its natural counterpart (Iyer et al. 2009) and is the most stable form of folate that is often used for dietary supplementation and fortification (Wright et al. 2007, Zhao et al. 2009). General populations in North America have achieved adequate folate intakes since implementation of the mandatory folate fortification policy in 1998 (Bailey et al. 2010). However, a low level of serum folates has been reported in patients with obesity, type 2 diabetes and NAFLD. Dysregulation of folate-dependent one-carbon metabolism has been implicated in NAFLD-related comorbidities such as obesity, type 2 diabetes, and metabolic syndrome (Mahabir et al. 2008, da Silva et al. 2014, Nilsson et al. 2015, Xia et al. 2018). Therefore, folic acid supplementation may be beneficial in patients with metabolic diseases such as obesity and NAFLD. However, the hepatoprotective effect of folate in NAFLD is poorly understood. The overall objective of my research is to investigate the role of folate in the context of diet-induced NAFLD. 


\subsection{Non-alcoholic fatty liver disease (NAFLD)}

\subsubsection{Epidemiology of NAFLD}

Non-alcoholic fatty liver disease (NAFLD) is the leading cause of chronic liver disease that affects both adults and children worldwide (Hedley et al. 2004). It is estimated that 83.1 million people in the world suffer from NAFLD (Estes et al. 2018). High incidence rates of NAFLD are reported in South America (31\%), the Middle East (32\%), Asia (27\%), United States of America (24\%) and Europe (23\%). In contrast, NAFLD is less prevalent in Africa (14\%) (Younossi et al. 2016, Younossi et al. 2018). The rising incidence of NAFLD is attributed to the global increase of obesity and type 2 diabetes in recent years (Loomba et al. 2013). It is predicted that 100.9 million people will exhibit NAFLD by 2030 (Estes et al. 2018). While only 20 to 30\% of the general population is afflicted with NAFLD, up to 70 to $90 \%$ of patients with obesity and type 2 diabetes are affected by this disease (Loomba et al. 2013, Ahmed 2015). NAFLD patients are likely to develop obesity and its associated metabolic comorbidities such as hyperlipidemia, hyperglycemia, insulin resistance and hypertension (Marchesini et al. 1999). Moreover, approximately $60 \%$ of NAFLD patients develop non-alcoholic steatohepatitis (NASH), the advanced form of NAFLD (Younossi et al. 2016). While 10 to $29 \%$ of NASH patients develop cirrhosis within a 10 year period (Argo et al. 2009), up to 4 to $27 \%$ of patients with NASHrelated cirrhosis progress to hepatocellular carcinoma (HCC) (Starley et al. 2010). NASH is currently the second most common cause of liver transplantation (Wong et al. 2014, Wong et al. 2015). Although pathogenesis of NAFLD may lead to end-stage liver diseases such as cryptogenic cirrhosis, chronic liver failure and HCC (Angulo 2002), the primary cause of mortality among patients with NAFLD is cardiovascular disease (Rafiq et al. 2009). It was reported that the incidence of cardiovascular deaths is greater than liver-related mortality in 
NAFLD patients (Younossi et al. 2016). However, NAFLD-related liver mortality in patients is projected to increase due to the growing rates of obesity and diabetes (Estes et al. 2018).

\subsubsection{Risk factors of NAFLD}

i) Dietary and metabolic risk factors

NAFLD manifests when caloric intake exceeds the rate of energy expenditure, which leads to perturbations in lipid and glucose metabolism (Fabbrini et al. 2010, Birkenfeld et al. 2014). The majority of NAFLD patients exhibit comorbidities of the metabolic syndrome, such as hyperglycemia, hypertension, dyslipidemia, low levels of high density lipoprotein (HDL), and central obesity (also known as visceral adiposity) (Marchesini et al. 2001, Farrell et al. 2006, Anstee et al. 2013). Dietary composition significantly influences the development of NAFLD and its associated metabolic comorbidities (Wree et al. 2013). Increased consumption of dietary fats and carbohydrates induces obesity and insulin resistance, which are among the most significant risk factors of NAFLD (Angulo 2002, Softic et al. 2016). Patients with obesity typically exhibit a body mass index (BMI) greater than or equal to $30 \mathrm{~kg} / \mathrm{m}^{2}$. Obesity increases the prevalence of NAFLD, but fatty liver may also manifest in individuals whose BMI do not fall within the obese range (Younossi et al. 2012, Younossi et al. 2016). Although these individuals are classified as non-obese, they frequently exhibit central obesity. Central obesity, rather than increased BMI, is associated with insulin resistance (Farrell et al. 2006). Insulin resistance is an independent risk factor of NAFLD (Marchesini et al. 1999, Monetti et al. 2007). However, it is unclear whether insulin resistance is a cause or consequence of NAFLD (Cohen et al. 2011). Current evidence on the relationship between NAFLD and insulin resistance is contradictory. Previous studies have demonstrated that impaired insulin signaling promotes development of 
steatosis in patients (Bhargava et al. 2004, Semple et al. 2009). On the other hand, it was reported that steatosis may develop without insulin resistance (Monetti et al. 2007). This suggests that although insulin resistance can cause steatosis, steatosis does not always cause insulin resistance (Cohen et al. 2011).

ii) Genetic risk factors

Asides from metabolic risks, non-modifiable risk factors such as age, gender and ethnicity also influence the susceptibility to fatty liver disease. Recent studies indicate that NAFLD is frequently observed in the elderly population, as well as in males compared to females (Amarapurkar et al. 2007, Chen et al. 2008, Vernon et al. 2011). Men are more likely to accumulate abdominal fat, which may increase their risk towards NAFLD development (Schwimmer et al. 2005). Moreover, it was reported that Hispanics and Asians are more likely to develop fatty liver compared to individuals of African descent (Browning et al. 2004). Hispanic individuals often possess a genetic variant in the patatin-like phospholipase domain-containing 3 (PNPLA3) gene (Romeo et al. 2008, Birkenfeld et al. 2014). PNPLA3 (also known as adiponutrin) is a lipase that is abundantly expressed in adipocytes and hepatocytes (Kotronen et al. 2009). This enzyme is highly active during increased intracellular lipid storage (Sookoian et al. 2012). Patients that carry the genetic variant of PNPLA3 exhibit a two-fold increase in hepatic triglyceride content, which confers susceptibility towards NAFLD development (Cohen et al. 2011). NAFLD is also observed in healthy, lean subjects that exhibit one of the two common gene variants in the apolipoprotein C3 (ApoC3) gene (Jenkins et al. 2004, Petersen et al. 2010, Birkenfeld et al. 2014). The ApoC3 protein is a component of very low-density lipoproteins (VLDL) that controls lipid distribution to tissues through regulation of lipoprotein lipase. This gene variant does not directly cause steatosis, but predisposes lean individuals to 
hepatic insulin resistance and NAFLD (Petersen et al. 2010, Birkenfeld et al. 2014). In addition, mutations in other genes regulating lipid metabolism can also enhance the risk of NAFLD (Birkenfeld et al. 2014).

\subsubsection{Natural history of NAFLD}

NAFLD represents a broad spectrum of liver diseases that develop independently of alcohol consumption. NAFLD ranges from steatosis to NASH and cirrhosis (Fig 1.1) (Tiniakos et al. 2010, Cohen et al. 2011). The term NASH was coined by Ludwig et al. in 1980 to describe patients that exhibited fatty liver with necroinflammation in the absence of alcohol consumption (Ludwig et al. 1980). Simple steatosis or "fatty liver" is generally a benign condition but in some cases, it may progress to NASH (Day 2005, Cohen et al. 2011). It remains unclear why some patients solely display steatosis while in others, the disease advances to aggressive forms of NAFLD such as NASH and cirrhosis (Cohen et al. 2011). It was reported that insulin resistance and other features of the metabolic syndrome are independently associated with the advanced forms of NAFLD (Dixon et al. 2001). Cirrhosis can ultimately advance to hepatocellular carcinoma (HCC) (Farrell et al. 2006). There is currently no specific test or biomarkers available to predict the progression of NAFLD. Most NAFLD patients appear asymptomatic until cirrhosis develops (Hashimoto et al. 2013). Histological evaluation of a liver biopsy is the most accurate method to identify and distinguish the stages of NAFLD (Kleiner et al. 2005, Wieckowska et al. 2008). 


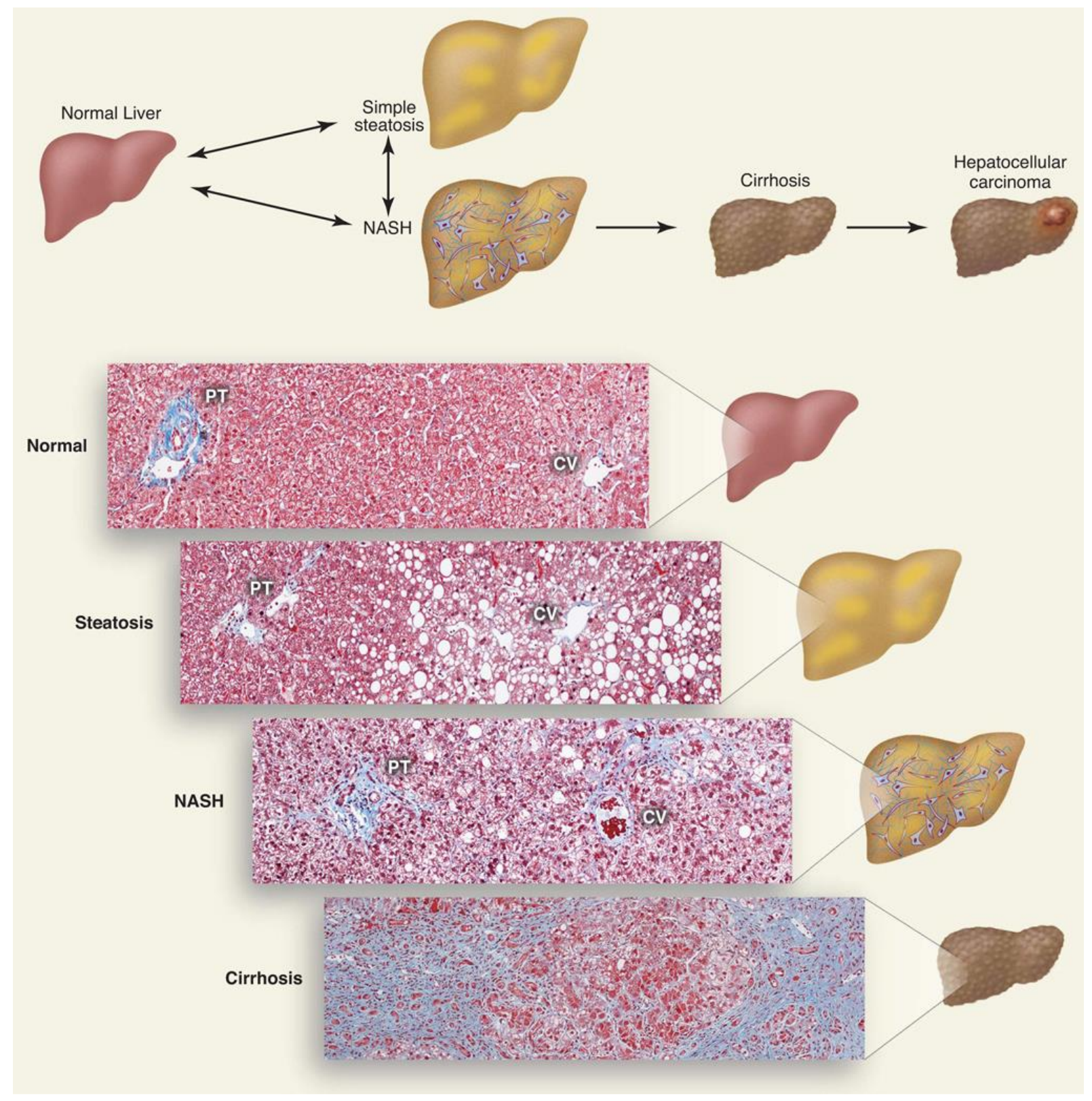

\section{Figure 1.1. The disease spectrum of NAFLD}

A schematic that illustrates the disease spectrum of NAFLD. The liver histological images represent each stage of NAFLD. PT, portal triad; CV, central vein. (Image based on Cohen et al. 2011. Science) 


\subsubsection{Disease spectrum of NAFLD}

\section{i) Steatosis}

Steatosis is the main histopathological feature of NAFLD. It is defined as intracytoplasmic deposition of lipids, predominantly as triglycerides, in greater than 5\% of hepatocytes (Brunt et al. 2010, Tiniakos et al. 2010). Steatosis is often presented as a mixture of large and small lipid vacuoles in liver tissue. These vacuoles are usually macrovesicular in which a single lipid droplet displaces the nucleus to the cell periphery (Tandra et al. 2011, Yeh et al. 2014). In contrast, microvesicular steatosis occurs when numerous tiny fat vesicles accumulate in the cytoplasm of hepatocytes. Predominant microvesicular steatosis is uncommon in NAFLD patients (Brunt et al. 2010, Tiniakos et al. 2010). However, the presence of microvesicular steatosis is significantly associated with histological features of NASH such as advanced fibrosis and hepatocyte injury. In contrast, macrovesicular steatosis can manifest independently of fibrosis or cirrhosis (Yeh et al. 2014). Steatosis develops when lipogenesis and fatty acid uptake exceed the rate of triglyceride export and fatty acid $\beta$-oxidation in the liver (Fabbrini et al. 2010, Cohen et al. 2011). Hepatic expression of genes involved in de novo lipogenesis are significantly elevated in patients with steatosis (Kohjima et al. 2007). In addition, the expression of fatty acid transport proteins (FATPs) is also upregulated in the liver of NAFLD patients (Westerbacka et al. 2007). Increased adipose tissue lipolysis accelerates fatty acid uptake by FATPs and contributes to the development of hepatic insulin resistance (Doege et al. 2008, Fabbrini et al. 2008). While the inhibitory effect of insulin on glucose production is disrupted, insulin's stimulatory effect on lipogenesis is maintained (Brown et al. 2008). In general, lipogenesis contributes to less than 5\% of hepatic triglyceride content in healthy individuals. However, $26.1 \%$ of triglycerides stored in the liver are derived from lipogenesis in patients with fatty liver. Such an increase in lipid 
biosynthesis may contribute to hepatic lipid accumulation in NAFLD patients (Donnelly et al. 2005, Lambert et al. 2014). Although mitochondrial oxidation of fatty acids and triglyceride export by very low-density lipoproteins (VLDL) are upregulated in patients with fatty liver, these processes cannot restore metabolic homeostasis in the liver (Sanyal et al. 2001, Adiels et al. 2006, Fabbrini et al. 2008). Steatosis is often self-limiting, but in some cases, it might advance to NASH, the progressive form of NAFLD (Cohen et al. 2011).

ii) Non-alcoholic steatohepatitis (NASH)

The minimum requirement for diagnosis of NASH includes the presence of histopathological lesions such as macrovesicular steatosis, lobular inflammation and ballooned hepatocytes (Tiniakos et al. 2010, Yeh et al. 2014). Hepatic inflammation is depicted by clusters of inflammatory cell aggregates which are composed of lymphocytes, eosinophils, leukocytes and kupffer cells (resident liver macrophages) (Kleiner et al. 2012). Patients with NASH often exhibit mild lobular inflammation, which is accompanied by inflammation around the hepatic portal tracts (portal inflammation). Varying degrees of portal inflammation in NASH correlates with insulin resistance and enhanced disease severity (Tiniakos et al. 2010). Hepatocyte ballooning is a form of hepatocellular injury that is characterized by cell swelling. This histopathological lesion is an essential feature in NAFLD patients that distinguishes NASH from steatosis (Yeh et al. 2014). Asides from hepatocyte ballooning, cellular injury is also present as apoptotic bodies and lytic necrosis (Brunt et al. 2010, Tiniakos et al. 2010). Although liver fibrosis is not an essential feature for NASH diagnosis, it is frequently detected in the liver of NASH patients (Tiniakos et al. 2010, Yeh et al. 2014). The presence of advanced hepatic fibrosis increases the risk for cirrhosis and liver complications such as hepatocellular carcinoma (HCC) and therefore, is a strong indicator of long-term mortality in NAFLD patients (Angulo et al. 
2007, Angulo et al. 2015). The mechanisms that drive the progression of steatosis to NASH are still incompletely understood (Haas et al. 2016). Increased concentrations of hepatic free fatty acids can induce lipotoxic cell injury (Alkhouri et al. 2009). Reactive oxygen species (ROS) generated from excessive fatty acid oxidation may contribute to hepatic oxidative injury and apoptosis (Farrell et al. 2006).

iii) Cirrhosis and hepatocellular carcinoma

While the progression of steatosis to NASH is reversible, NASH may irreversibly advance to cirrhosis in which hepatic tissue is replaced with collagenous fibrotic lesions (Cohen et al. 2011). Hepatic stellate cells are activated in response to liver injury and stimulate collagen production and deposition in the liver (Brunt et al. 2010, Cohen et al. 2011). The presence of liver cirrhosis is frequently accompanied by hepatocyte ballooning degeneration in NAFLD patients (Kessoku et al. 2014). NAFLD patients with liver cirrhosis are increasingly susceptible to the development of portal hypertension and hepatocellular carcinoma (HCC), a major cause of liver-related mortality (Starley et al. 2010, Kessoku et al. 2014). Obesity and diabetes are identified as major risk factors for HCC (Regimbeau et al. 2004). A previous study reported that $62 \%$ of cirrhotic patients developed liver-related complications including $\mathrm{HCC}$, while $33 \%$ of the patients died from liver failure (Hui et al. 2003). Moreover, hepatic cirrhosis independently increases the overall death risk in NAFLD patients by threefold (Adams et al. 2005). Due to increased prevalence for advanced stages of NAFLD, NASH-related cirrhosis is predicted to become the primary cause of liver transplantation in the near future (Wree et al. 2013). 


\subsubsection{Pathogenesis of NAFLD}

The pathogenesis of NAFLD is complex and not completely understood. The two-hit hypothesis was initially proposed to describe NAFLD pathogenesis. This theory suggests that perturbations in lipid metabolism leads to steatosis (first hit), which sensitizes the liver to secondary hits such as inflammation, oxidative stress, and cell injury (Day 2005). However, steatosis may not always precede inflammation. It was previously reported that inflammation could occur before lipid accumulation in NASH patients (Tiniakos et al. 2010). Furthermore, the two-hit theory does not account for many other metabolic changes that occur in NAFLD. This lead to the development of the multiple-parallel hit model, which suggests that several factors such as insulin resistance, lipotoxicity, oxidative stress, inflammation, gut-derived endotoxins, adipokines, or genetic factors may simultaneously induce NAFLD (Tilg et al. 2010). Hepatic lipotoxicity caused by an excessive flux of free fatty acids (particularly saturated fatty acids) or free cholesterol to the liver stimulates the generation of toxic lipid metabolites, which may contribute to hepatic oxidative stress and inflammation (Neuschwander-Tetri 2010). Oxidative damage is an important cause of hepatocyte injury and death (Jaeschke 2000). Increased $\beta$-oxidation of free fatty acids is a major source of ROS that triggers activation of inflammatory response in NAFLD (Satapati et al. 2015). In addition, inflammatory mediators derived from adipose tissue and intestine can stimulate liver damage and fibrosis (Tilg et al. 2010). Enhanced intestinal permeability to bacterial-derived products was shown to induce lipid accumulation and hepatic inflammation in NAFLD patients (Miele et al. 2009, Rahman et al. 2016). While genetic factors may not significantly contribute to NAFLD development, certain genetic variations may increase the susceptibility to NAFLD. Overall, multiple insults may occur in parallel rather than in 
consecutive order leading to the development of NAFLD. This suggests that the multiple-hit hypothesis is most accurate for describing the pathogenesis of NAFLD.

\subsubsection{Animal and cell models for studying NAFLD}

A number of nutritional and genetic-based animal models have been established to study the onset and pathogenesis of NAFLD (Hebbard et al. 2011, Takahashi et al. 2012). These animal models closely reflect the histopathology and pathophysiology of the disease (Larter et al. 2008, Takahashi et al. 2012). However, there is currently no single animal model that completely reflects the histopathology of the entire NAFLD spectrum (Hebbard et al. 2011). In addition, cell models have been developed to investigate the molecular mechanisms involved in the progression of the disease (Kanuri et al. 2013).

i) High-fat diet model

High-fat diet feeding in mice induces a metabolic phenotype that closely resembles that observed in NAFLD patients (Buettner et al. 2007, Takahashi et al. 2012). The C57Bl/6 mouse model is commonly used to study NAFLD because it is most susceptible to diet-induced obesity and displays significant body weight gain (Collins et al. 2004, Winzell et al. 2004). Diets enriched in $30-75 \%$ kcals of fat can promote development of steatosis and insulin resistance, as well as hyperglycemia and dyslipidemia (Buettner et al. 2007, Kanuri et al. 2013). Although body weight gain in rodents is observed after 2 weeks of high-fat diet feeding, the diet-induced phenotype is most apparent after 4 weeks or more (Buettner et al. 2007). A significant elevation in plasma glucose and insulin were detected in mice fed a high-fat diet for 4 weeks (Sato et al. 2010). Furthermore, mice developed steatosis after 4 to 8 weeks of high-fat diet feeding (Asgharpour et al. 2016). However, high-fat diet feeding for 35-50 weeks is necessary to induce 
NASH in mice (Ito et al. 2007). Chronic consumption of high-fat diets stimulates oxidative stress and inflammation in mouse liver, but does not cause severe degrees of fibrosis or liver injury as observed in the mice fed a methionine-choline deficient (MCD) diet (Omagari et al. 2008, Lanthier et al. 2011). However, fructose, cholesterol or cholate in the presence of a high-fat diet, exacerbates the features of NASH such as steatosis, inflammation, fibrosis and oxidative stress (Kanuri et al. 2013, Luo et al. 2016). Dietary fat composition is also an important factor that determines development of NAFLD. High-fat diets containing a high saturated fat content (coconut oil, lard, and tallow) effectively induces metabolic abnormalities and histological features of NAFLD compared to unsaturated fats (fish oils) (Buettner et al. 2007).

ii) Genetic animal models

The $o b / o b$ and $d b / d b$ mice, as well as $f a / f a$ zucker rats are the most common genetic models used to study NAFLD (Kanuri et al. 2013). Leptin signaling is impaired in these animals due to mutations in the leptin gene or receptor, therefore, they are obese and insulin resistant (Takahashi et al. 2012). The $o b / o b$ mice are leptin deficient and hyperphagic due to a point mutation in the leptin gene (Bray et al. 1979, Anstee et al. 2006). These mice develop obesity, hyperlipidemia and insulin resistance when consuming a normal chow diet (Mayer et al. 1951). A disadvantage of the $o b / o b$ mouse model is that it does not mimic the human condition. Rather than being leptin deficient, humans typically display normal or elevated serum leptin levels (Uygun et al. 2000, Chalasani et al. 2003). In contrast, the $d b / d b$ mice and $f a / f a$ rats are leptin resistant and display elevated leptin levels in the circulation (Godbole et al. 1978, Chen et al. 1996, Oana et al. 2005). These genetically modified animals exhibit steatosis, as well as hyperinsulinemia and hyperlipidemia (Bray et al. 1979, Chen et al. 1996, Wortham et al. 2008). However, they do not spontaneously develop steatohepatitis. Exposure to a low dose of endotoxins or consumption of a 
methionine-choline deficient (MCD) diet is required to induce steatohepatitis in these rodents (Yang et al. 1997, Brix et al. 2002, Wortham et al. 2008).

iii) Methionine-choline deficient (MCD) model

The MCD model is commonly used to study NASH. The MCD diet is enriched in sucrose and fat (40\% sucrose, 10\% fat), but lacks methionine and choline (Anstee et al. 2006, Hebbard et al. 2011). Methionine and choline are essential micronutrients that are required for triglyceride export from hepatocytes. Consumption of the MCD diet impairs hepatic $\beta$-oxidation, as well as attenuates production and secretion of VLDL. As a result, there is increased lipid deposition in the liver (Yao et al. 1990, Anstee et al. 2006). Mice fed a MCD diet develop severe liver damage, steatosis, inflammation and fibrosis within 8 to 10 weeks. These histopathological features resemble those observed in NASH patients (Takahashi et al. 2012). However, the metabolic phenotype of mice fed a MCD diet does not correlate with human NAFLD. MCD-fed mice experience significant weight loss, which is associated with low fasting blood glucose, as well as reductions in plasma glucose, insulin and cholesterol levels (Rinella et al. 2008, Hebbard et al. 2011).

iv) Cell models

Primary cell cultures and immortalized cell lines are used to investigate the mechanisms of NAFLD pathogenesis (Kanuri et al. 2013). Primary human hepatocytes, kupffer cells, stellate cells and sinusoidal epithelial cells are clinically relevant cell models of NAFLD (GomezLechon et al. 2004, Dambach et al. 2005). However, use of these human cell models for research is extremely difficult due to ethical issues and limited availability of liver samples. Primary rodent hepatocytes closely reflect the conditions in human hepatocytes, but establishing methods for isolation may be an experimental challenge (Kanuri et al. 2013). Immortalized human 
hepatocyte cell lines such as HepaRG or HepG2 are widely used to study NAFLD instead of primary hepatocytes because they are easier to grow and culture. Moreover, these immortalized cells typically display a stable phenotype that closely resembles that in primary hepatocytes (Chavez-Tapia et al. 2011, Kanuri et al. 2013). However, one disadvantage is that expression of certain enzymes and proteins may be different between immortalized and primary hepatocytes (Kanuri et al. 2013). Asides from immortalized human hepatocytes, RAW 264.7 macrophages and alpha mouse liver-12 (AML-12) cells are utilized as models of kupffer cells and murine hepatocytes respectively. These cells are commonly used in co-culture to investigate cell to cell interaction during the progression of NAFLD (Spruss et al. 2011, Kanuri et al. 2013).

\subsubsection{Diagnosis of NAFLD}

There are currently no reliable biomarkers or serological tests available for accurate diagnosis of NAFLD (Hadizadeh et al. 2017). Although NAFLD is a common cause of elevated serum alanine aminotransferase (ALT) levels, some patients with NAFLD display normal liver enzyme levels (Fracanzani et al. 2008). The minimal diagnostic criterion for NAFLD is the presence of steatosis that develops independently of any significant alcohol influence and is not caused by gene mutations or other secondary liver disorders such as hepatitis (Brunt et al. 2010). In recent years, imaging tests such as transabdominal ultrasound, non-contrast computed tomography (CT) scan and magnetic resonance imaging (MRI) have been commonly used to detect fatty liver (Ahmed 2015). These techniques are relatively safe and non-invasive but fail to identify and differentiate the stages of NAFLD that reflect progression of the disease (Chalasani et al. 2012). The stages of hepatic fibrosis can be assessed by hepatic elastography, which measures liver stiffness performed by ultrasound or MRI. However, this technique is rather expensive and not 
well-established (Afdhal 2012, Chalasani et al. 2012). Histological evaluation of a liver biopsy remains to be the gold standard for assessing steatosis, as well as for grading stages of hepatic inflammation and fibrosis (Kleiner et al. 2005). Despite that a liver biopsy is an invasive procedure and potentially harmful to patients, it is necessary for evaluation of histological lesions that distinguish steatosis from NASH and cirrhosis (Brunt et al. 2010). It should be noted though, that a liver biopsy cannot be considered as a screening tool for NAFLD in population studies because this procedure is highly invasive and suffers from sampling variability. Since histological lesions among all NAFLD patients are not evenly distributed throughout the liver, a liver biopsy may result in a large sampling error and inaccurate staging of the disease (Ratziu et al. 2005, Brunt et al. 2010).

\subsubsection{NAFLD management}

Exercise and dietary restriction are the most safe and effective strategies for NAFLD management (Thoma et al. 2012). These lifestyle modifications promote weight loss and can lead to improvement in liver function and steatosis in patients (Chalasani et al. 2012). Although minimal weight losses (3-5\%) in patients is sufficient to reduce hepatic lipid accumulation, greater than $7 \%$ weight reduction is required to resolve histological features associated with NASH (Glass et al. 2015, Hannah et al. 2016). Overall weight loss is beneficial for patients with NAFLD, but it is difficult to achieve and sustain (Ahmed 2015). Patients who receive bariatric surgery have profound weight loss, which is associated with improved liver histology and insulin sensitivity (Mechanick et al. 2009). However, bariatric surgery is not recommended for NAFLD treatment due to concerns regarding hepatic failure in cirrhotic patients with rapid weight loss (Grimm et al. 1992). The pathogenesis of NAFLD is incompletely understood and therefore, 
there are currently no therapeutic drugs approved for treatment of this disease. Several therapeutic agents that target certain metabolic risk factors have been proposed for treatment of NAFLD (Chalasani et al. 2012). Metformin, a first line anti-diabetic agent, reduces insulin resistance and liver ALT levels but has limited effects on alleviating inflammation and steatosis in NAFLD patients (Bugianesi et al. 2005). Thiazolidinediones such as pioglitazone and rosiglitazones are also insulin sensitizers and have been shown to effectively ameliorate liver function and histology in patients with NASH (Neuschwander-Tetri et al. 2003, Belfort et al. 2006). However, their long-term safety and efficacy for NAFLD treatment has not been established (Chalasani et al. 2012). Oxidative stress is a key mediator of cell injury during NASH (Seki et al. 2002). While treatment with antioxidants such as vitamin $\mathrm{E}$ have been shown to resolve steatosis, inflammation and hepatocyte ballooning, further investigation is required to determine the effectiveness of antioxidants to treat NAFLD (Sanyal et al. 2004). Moreover, NAFLD patients are at higher risk for cardiovascular disease and therefore, statins have been used to treat dyslipidemia and improve cardiovascular outcomes in these patients (Athyros et al. 2010). The use of statins appears to be safe and effective for treatment of lipid abnormalities in NAFLD (Chalasani et al. 2012). Although several agents have been assessed for NAFLD treatment, there is no single agent that can treat all metabolic risk factors associated with this disease. Emerging therapies such as non-antifibrotic and antifibrotic drugs are currently under clinical investigation for treatment of NAFLD. Researchers are searching for therapeutic agents that can target multiple pathways that are associated with NAFLD pathogenesis (Younossi et al. 2018). 


\subsection{Lipid metabolism}

2.2.1. Absorption of dietary lipids and delivery to peripheral tissues

Triglycerides and cholesterol are among the major types of lipids provided in the diet. The initial absorption of lipids occurs in the small intestine (Shiau 1981, Ros 2000). Triglyceride digestion to fatty acids is mainly mediated by pancreatic lipase. Fatty acids are subsequently emulsified by bile acids which facilitate absorption of lipid molecules by enterocytes (Ros 2000, Bechmann et al. 2012). Similarly, cholesterol is hydrolyzed by pancreatic esterases to its unesterified form for intestinal absorption ( $\mathrm{Lu}$ et al. 2001). Following absorption, free fatty acids are re-esterified to triglycerides mediated by diglyceride acyltransferase (DGAT), while re-esterification of cholesterol esters is catalyzed by acyl-coenzyme A: cholesterol acyltransferase (ACAT). These lipids are subsequently packaged into chylomicrons for delivery to peripheral tissues (Ros 2000). Chylomicrons are the major lipoproteins responsible for transport of dietary lipids from the intestine. While triglycerides in chylomicrons are directly distributed to liver, most dietary cholesterol enters the liver in the form of chylomicron remnants ( $\mathrm{Lu}$ et al. 2001). Hepatic cholesterol is subsequently packaged into VLDL and either secreted to the plasma or bile. Cholesterol in bile enters the enterohepatic circulation and is efficiently reabsorbed at the intestine and redistributed to the liver (Ros 2000, Lu et al. 2001).

\subsubsection{Triglyceride and cholesterol metabolism}

The liver is the major site for lipid metabolism. Hepatic fatty acids are primarily derived from the diet, endogenous lipid synthesis or adipose tissue lipolysis (Donnelly et al. 2005). The de novo lipogenesis is a process when fatty acids are synthesized endogenously from acetyl-CoA, a metabolite from carbohydrates. Hepatic fatty acid synthesis is regulated by acetyl-CoA 
carboxylase (ACC) and fatty acid synthase (FAS) (Bechmann et al. 2012). On the other hand, fatty acid uptake in hepatocytes is mainly mediated by fatty acid transport proteins (FATPs) (Newberry et al. 2003). Fatty acids are either stored in hepatocytes as triglycerides or oxidized within the mitochondria and peroxisomes to provide energy (Reddy et al. 2006). In the postprandial state, hepatic fatty acid oxidation is inhibited and de novo lipogenesis in hepatocytes is stimulated to promote triglyceride storage and export by VLDL. In contrast, $\beta$-oxidation in hepatocytes serves as a major source of energy during fasting or energy depleting conditions (Akkaoui et al. 2009). Aside from triglyceride metabolism, the liver also plays an important role in cholesterol homeostasis. The liver provides an abundant supply of cholesterol in the body (Dietschy et al. 1993, Lu et al. 2001). Hepatic cholesterol synthesis occurs in the endoplasmic reticulum and is regulated by 3-hydroxy-3-methylglutaryl coenzyme A (HMG-CoA) reductase, which catalyzes the rate-limiting step of cholesterol biosynthesis (Kaplan et al. 1985). Free cholesterol is converted to cholesterol esters, excreted to the circulation by lipoproteins (VLDL or HDL), or metabolized to bile acids in the liver (Arguello et al. 2015). In addition to endogenous cholesterol synthesis by the liver, animal foods such as red meat, poultry and dairy products are rich sources of dietary cholesterol. Bile acids are essential for intestinal absorption of dietary cholesterol, which is delivered to the liver by chylomicrons. Hepatic cholesterol is subsequently distributed to peripheral tissues by low-density lipoprotein (LDL) (Lu et al. 2001). The LDL receptor is responsible for cholesterol uptake (in the form of LDL) in hepatocytes via receptor-mediated endocytosis (Brown et al. 1981). Hepatic expression LDL receptors are regulated in response to cholesterol levels in the liver. High cholesterol levels attenuate LDL receptor function, while receptor-mediated cholesterol uptake in hepatocytes is stimulated during cholesterol depletion (Goldstein et al. 1984). 


\subsection{Carbohydrate metabolism}

\subsubsection{Carbohydrate digestion and absorption}

Carbohydrate digestion is mediated by intestinal epithelial cell enzymes. Absorption of carbohydrates in the form of monosaccharides (glucose, fructose and galactose) occurs in the gastrointestinal tract (Levin 1994). Glucose and galactose are phosphorylated to facilitate passive absorption by enterocytes, while fructose is absorbed in its free form (Dashty 2013). In contrast, active transport of carbohydrates by a carrier protein is coupled with the $\mathrm{Na}^{+} / \mathrm{K}^{+}$pump, which utilizes the $\mathrm{Na}^{+} / \mathrm{K}^{+}$gradient as an energy source. Dephosphorylation of glucose and galactose must occur prior to entry into the liver (Stevens et al. 1984). Galactose and fructose is converted to glucose for metabolism in the liver. Glucose uptake by hepatocytes is mediated by glucose transporter type 2 (Glut 2), while glucose transporter type 4 (Glut 4) is responsible for glucose transport in muscle and adipose tissue (Rencurel et al. 1996, Bryant et al. 2002). Glucose is immediately phosphorylated to glucose-6-phosphate, which is required for glucose metabolism in hepatocytes. This reaction is mediated by liver glucokinase, a glucose sensor that regulates hepatic glucose metabolism (Han et al. 2016).

\subsubsection{Glucose metabolism}

The liver is the main organ responsible for glucose metabolism. Depending on the metabolic state, glucose is either metabolized to pyruvate or stored as glycogen (Nordlie et al. 1999). During feeding, glucose is converted to pyruvate, which undergoes oxidative decarboxylation to produce acetyl-CoA. Acetyl-CoA serves as a substrate for mitochondrial oxidative phosphorylation to generate ATP (Han et al. 2016). However, glucose is converted to pyruvate via glycolysis in cells without mitochondria or subjected to ischemia. Glycolysis is a series of 
reactions that converts glucose to pyruvate, which in turn, is reduced to lactate for anaerobic generation of ATP. Under energy deplete conditions, glycogen phosphorylase catalyzes breakdown of glycogen to glucose (Rui 2014). Hepatic gluconeogenesis occurs when noncarbohydrate substrates such as pyruvate, lactate, glycerol, alanine or glutamine are synthesized to glucose. Glucose serves as a major source of energy for the brain and red blood cells (Nordlie

et al. 1999). Insulin and glucagon are among the key players that regulate glycolysis and gluconeogenesis. Insulin promotes hepatic glucose uptake and glycolysis, while suppressing glucose biosynthesis in the liver. In contrast, glucagon increases hepatic glucose production to provide an energy source for peripheral tissues (Han et al. 2016).

\subsection{AMP-activated protein kinase (AMPK)}

\subsubsection{Regulation of AMPK}

The AMPK is a serine/threonine protein kinase that modulates metabolic homeostasis in correspondence to cellular energy status. It is typically activated in response to exercise, starvation and cellular stress that depletes ATP levels (eg. increases in intracellular AMP) (Hardie et al. 2012). AMPK is comprised of three subunits: $\alpha, \beta$, and $\gamma$-subunit. Each of these subunits has many isoforms that are encoded by distinct genes. The catalytic $\alpha$-subunit exhibits a phosphorylation site (Thr-172) at the amino-terminus, while the regulatory $\gamma$-subunit contains 4 adenine nucleotide binding sites. While adenine binding site 2 is usually unoccupied, AMP is tightly bound at site 4 . Sites 1 and 3 on the AMPK- $\gamma$ subunit are exchangeable sites that have equal affinity to all adenine nucleotides (ATP, AMP, ADP). The $\beta$-subunit contains a carbohydrate binding module that promotes association with glycogen. This subunit serves as a scaffold that maintains the structure of the heterotrimeric AMPK protein (Hardie et al. 2012, 
Gowans et al. 2013). AMPK is activated upon phosphorylation on its catalytic site ( $\alpha$-subunit) and binding of AMP on its allosteric sites ( $\gamma$-subunit). While upstream kinases such as transforming growth factor- $\beta$-activating kinase (TAK1), calcium/calmodulin-dependent protein kinase kinase 2 (CaMKK2) and liver kinase B1 (LKB1) mediate phosphorylation of AMPK, protein phosphatases (PP2A and PP2C) are responsible for dephosphorylation of the kinase (Davies et al. 1995). Phosphorylation causes greater than 100-fold activation of AMPK. However, allosteric activation by AMP is crucial to maintain its phosphorylation. AMP binding protects AMPK from dephosphorylation (inactivation) and therefore, this enzyme is maintained in its activated state. In contrast, ATP competes with AMP for allosteric binding sites on AMPK and therefore, increased binding of ATP to AMPK attenuates the activity of this kinase. Although ADP is also considered an allosteric activator of AMPK, activation of AMPK by AMP is 10-fold more potent than that by ADP (Gowans et al. 2013). The relative increase in AMP levels is more prominent than ADP during energy depletion conditions. Other enzymes may respond to changes in energy status, but AMPK is the principal energy sensor that possesses widespread effects on cell metabolism and function (Hardie 2007).

\subsubsection{AMPK function}

The AMPK is a master regulator of metabolic homeostasis in the liver and skeletal muscle. In response to falling energy status, AMPK functions to restore energy balance by inhibiting energy consuming biosynthetic processes while promoting catabolism of macronutrients for energy production (Hardie et al. 2012). This kinase mediates metabolic changes through phosphorylation of various downstream proteins. Activation of AMPK attenuates hepatic fatty acid and cholesterol biosynthesis, which is mediated by phosphorylation (inactivation) of ACC1 
and HMG-CoA reductase, respectively (Hardie 2004). In addition, AMPK-dependent phosphorylation of sterol regulatory element binding protein-1c (SREBP-1c) and carbohydrate responsive element binding protein (ChREBP) suppresses hepatic lipogenic gene expression and therefore, decreases hepatic lipogenesis (Foretz et al. 2005). On the other hand, AMPK phosphorylation (inactivation) of ACC2 promotes fatty acid oxidation in the liver (Assifi et al. 2005). AMPK-mediated fatty acid oxidation prevents lipid accumulation and enhances insulin sensitivity in skeletal muscle (Watt et al. 2006). Increased insulin sensitivity stimulates glucose disposal by enhancing glucose uptake by Glut 4 in muscle cells (Buhl et al. 2001). AMPKmediated phosphorylation of phosphoenolpyruvate carboxykinase (PEPCK) and glucose-6phosphatase (G-6-Pase) suppresses activations of these enzymes and therefore, leads to inhibition of gluconeogenesis (Lochhead et al. 2000). Asides from its dominant role in glucose and lipid metabolism, AMPK also promotes energy conservation through regulation of cell cycle and membrane excitability (Imamura et al. 2001, Ikematsu et al. 2011). Since dysregulation of energy homeostasis perturbs metabolic function (Rui 2014), regulation of AMPK may have an important implication in metabolic disease.

\subsubsection{AMPK in health and disease}

AMPK is a key regulator of lipid and glucose metabolism, which is often perturbed in metabolic diseases such as obesity, type 2 diabetes, metabolic syndrome and NAFLD. Decreased AMPK activation is often associated with metabolic dysfunction (Viollet et al. 2006, Smith et al. 2016). It was reported that whole-body AMPK $\alpha 2$-specific knockout mice exhibited dyslipidemia, as well as impaired glucose tolerance and insulin sensitivity (Viollet et al. 2003, Andreelli et al. 2006). Treatment with 5-aminoimidazole-4-carboxamide ribonucleotide (AICAR), a direct 
activator of AMPK, was shown to reduce plasma triglyceride levels and adiposity in obese rodents (Bergeron et al. 2001). Similarly, constitutive activation of hepatic AMPK effectively lowers plasma glucose and triglyceride levels in diabetic, obese and high-fat diet fed rodents (Foretz et al. 2005, Cool et al. 2006, Yang et al. 2008). Activation of AMPK also abolishes hyperglycemia in diabetic $o b / o b$ and STZ-induced diabetic mice (Foretz et al. 2005). It was reported that chronic exposure to excess nutrients (glucose, lipids) leads to inhibition of AMPK (Saha et al. 2010, Coughlan et al. 2013). Various animal models of metabolic syndrome and obesity have decreased AMPK activity in the muscle and liver. Supporting evidence indicates that AMPK dysfunction predisposes obese individuals to development of insulin resistance and type 2 diabetes (Gauthier et al. 2011, Xu et al. 2012). Due to its widespread metabolic functions, AMPK has emerged as an attractive therapeutic target for metabolic disease. Pharmaceutical agents such as metformin and thiazolidinediones mediate insulin-sensitizing effects through indirect activation of AMPK and are currently used for the treatment of type 2 diabetes (Zhang et al. 2009). Small molecule AMPK activators have been demonstrated to effectively reduce plasma glucose and triglyceride levels in $o b / o b$ mice (Cool et al. 2006). Further studies are necessary to determine whether direct activation of AMPK is beneficial for treatment of metabolic disease.

\subsection{Inflammatory response}

\subsubsection{Role of the inflammatory response}

The inflammatory response is the body's innate defense mechanism against tissue injury or infection (Hotamisligil 2006, Lawrence et al. 2007). Circulating white blood cells such as neutrophils, monocytes and lymphocytes are recruited to the site of injury in response to 
pathological stimuli such as invading pathogens and foreign antigens (Luster et al. 2005). These immune cells produce and release vasoactive and inflammatory mediators that cause swelling, redness and pain (Larsen et al. 1983). The inflammatory response following tissue injury is essential for wound healing and tissue repair (Martin et al. 2005). The liver is the primary immunological organ that is exposed to antigen-rich blood delivered from the gastrointestinal tract. This organ is comprised of parenchymal hepatocytes (60-80\%) and non-parenchymal immune cells (20-40\%) such as kupffer cells (liver resident macrophages), endothelial cells, lymphocytes (natural killer cells, T cells and B cells), biliary cells and stellate cells (Racanelli et al. 2006). Hepatocytes and kupffer cells are the key players of the innate inflammatory response in the liver (Gao et al. 2008). Inflammation is usually a self-limiting process (Lawrence et al. 2001). Asides from production of pro-inflammatory cytokines that drives inflammation, liver parenchymal and immune cells also generate anti-inflammatory cytokines that control the proinflammatory response (Lawrence et al. 2001). However, hepatic inflammation persists when production of pro-inflammatory cytokines is aberrantly upregulated or anti-inflammatory function is impaired (Lawrence et al. 2007). This leads to a state of chronic inflammation, which is often associated with the development of metabolic disease (Bieghs et al. 2013).

\subsubsection{Chronic inflammation in metabolic disease}

Chronic or low-grade inflammation is a key feature of obesity, type 2 diabetes and fatty liver disease (Lumeng et al. 2011). Enhanced caloric intake perturbs metabolic homeostasis and induces chronic inflammation, particularly in metabolic tissues such as the liver and adipose tissue (Hotamisligil et al. 2008). Adipose tissue is the major site for lipid storage under physiological conditions. Adipocytes monitor energy storage and stimulate the production of 
pro-inflammatory cytokines in response to excessive lipid accumulation in adipose tissue (Xu et al. 2003). Overproduction of pro-inflammatory mediators in the adipose tissue during obesity contributes to the development of hepatic insulin resistance and chronic inflammatory state in the liver (Cai et al. 2005). In addition, increased flux of free fatty acids from the adipose tissue to the liver induces hepatic lipotoxic injury by stimulating the expression of inflammatory cytokines in the liver (Neuschwander-Tetri 2010). Hepatic inflammation is a main mediator of liver tissue damage, which promotes fibrogenesis and the development of hepatocellular carcinoma (HCC) (Park et al. 2010). Dysregulation of inflammatory cytokine production may eventually trigger hepatocyte apoptosis (Schattenberg et al. 2011). Moreover, inhibition of inflammatory signaling in hepatocytes has been demonstrated to improve hepatic insulin sensitivity (Arkan et al. 2005). Therefore, attenuating inflammatory response in the liver may be beneficial to reduce insulin resistance and prevent chronic inflammation associated with metabolic disease.

\subsubsection{Inflammatory signaling by nuclear factor- $\mathrm{\kappa B}(\mathrm{NF}-\kappa \mathrm{B})$}

The transcription factor NF- $\mathrm{kB}$ is the master regulator of the inflammatory response (Baker et al.

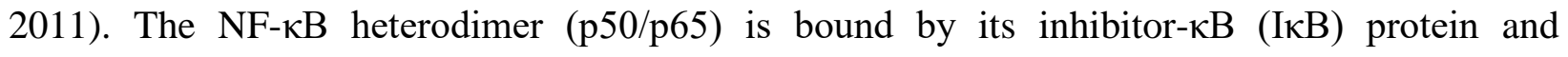
localized in the cytoplasm under normal physiological conditions. In response to foreign stimuli,

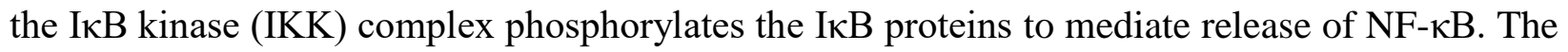
$\mathrm{NF}-\kappa \mathrm{B}$ protein subsequently translocates to the nucleus and triggers the expression of genes encoding for inflammatory cytokines such as interleukin-6 (IL-6) and tumor necrosis factor- $\alpha$ (TNF- $\alpha$ ) (Hayden et al. 2008, Christian et al. 2016). Although NF-kB also plays an important role in regulating the expression of genes involved in cell proliferation and cell death (Hayden et al. 2012), dysregulation of NF-kB activity is associated with chronic inflammation in metabolic 
disease (Gasparini et al. 2012). It was reported that diet-induced obesity in mice was associated with chronic activation of NF- $\mathrm{BB}$ in the liver and adipose tissue (Cai et al. 2005, Kwon et al. 2014). This leads to increased production of inflammatory cytokines, which may further aggravate the inflammatory response by promoting infiltration of immune cells such as neutrophils, eosinophils and macrophages in metabolic tissues (Xu et al. 2003, Hotamisligil 2006). Therefore, inactivation of NF- $\kappa \mathrm{B}$ may be a promising strategy to regulate inflammatory response during the onset of metabolic disease.

\subsection{Role of vitamins in metabolic disease / NAFLD}

Vitamins are essential micronutrients that play an important role in maintaining liver metabolism. There are two classes of vitamins: fat-soluble and water-soluble vitamins. Emerging studies are investigating the use of these micronutrients as alternative therapies for NAFLD and its associated metabolic diseases. Since vitamin E (lipid-soluble tocopherol) exhibits both antiinflammatory and antioxidant activity, it has been suggested that this vitamin may be beneficial for treatment of NAFLD (Veena et al. 2014). However due to safety concerns with long-term use of vitamin E, it is not yet recommended as a therapy for NAFLD (Chalasani et al. 2012). Dysregulation of lipid metabolism as observed in NAFLD is often associated with perturbations in one-carbon metabolism. The water-soluble B-complex vitamins such as cobalamin (B12), riboflavin (B2), and folate (B9) are essential cofactors for one-carbon metabolism reactions (da Silva et al. 2014). Among these micronutrients, folate (in the form of tetrahydrofolate) serve as a catalytic substrate for one-carbon transfer reactions that contribute to the generation of methyl groups derived from catabolism of sarcosine, serine, dimethylglycine, and glycine (Tibbetts et al. 2010, Stover et al. 2011). Under physiological conditions, the methyl group from folate (in the 
form of 5-methyltetrahydrofolate; 5-MTHF) is transferred to cobalamin (vitamin B12), which in turn, transfers the methyl group to homocysteine to form methionine. This reaction is mediated by methionine synthase (Purohit et al. 2007). Methionine is an important precursor of $S$ adenosylmethionine (SAM), a major methyl donor for cell metabolism reactions. Asides from folate, cobalamin is an essential cofactor that is required for transfer of methyl groups from 5MTHF to its substrates. Deficiency of cobalamin results in accumulation of 5-MTHF and can impair folate metabolism (Scott et al. 1994). Alternatively, another major source of methyl groups that primarily obtained from the diet is choline (Tibbetts et al. 2010). Choline is oxidized to betaine, which serves as a methyl donor for remethylation of homocysteine to methionine via betaine hydroxymethyltransferase (BHMT). This reaction generally occurs when methionine synthase activity is compromised, which can be due to chronic ethanol exposure (Purohit et al. 2007). Another product of the reaction catalyzed by BHMT is $N, N$-dimethylglycine (DMG), which serves as an important source of one-carbon units alongside glycine, serine and sarcosine (Tibbetts et al. 2010). One-carbon metabolism plays an essential role in many cellular processes including methylation, which is important in maintaining hepatic lipid metabolism (da Silva et al. 2014). Therefore, dysregulation of folate-dependent one-carbon metabolism may contribute to development of metabolic disease such as obesity and NAFLD (da Silva et al. 2014, Sid et al. 2017). 


\subsection{Folate}

\subsubsection{Folate and Folic acid}

Folate refers to a group of water-soluble B9 vitamins that serve as co-enzymatic substrates for one-carbon transfer reactions. Folate-dependent one-carbon transfer reactions are important for nucleic acid biosynthesis, methylation reactions, and sulfur-containing amino acid metabolism (Tibbetts et al. 2010, Stover et al. 2011). Mammals lack the enzymatic capacity to synthesize folates; therefore, the intake of dietary folates is essential to meet their physiological requirements (Lucock 2000, Zhao et al. 2009). The recommended dietary allowance (RDA) for folate in healthy individuals is $400 \mu \mathrm{g}$ of dietary folate equivalents (DFEs) per day. In pregnant women, the RDA increases to $600 \mu \mathrm{g}$ of DFEs per day to satisfy fetus requirements (Institute of Medicine Standing Committee on the Scientific Evaluation of Dietary Reference et al. 1998). Although folate is widely distributed in a variety of foods, animal liver and dark green leafy vegetables are the most abundant sources of naturally occurring folates (Lucock 2000, Zhao et al. 2009). Dietary folates are often in the reduced and polyglutamated forms (Wright et al. 2007, Zhao et al. 2009). In contrast to its reduced form, folic acid is an oxidized monoglutamate with higher bioavailability than its natural counterpart (Iyer et al. 2009). Folic acid is the synthetic (stable) form of folate that is used for dietary supplementation and fortification (Wright et al. 2007, Zhao et al. 2009). In addition to dietary folate and folic acid, folate-producing bacteria in the colon and the proximal small intestine may serve as an endogenous source of folates (Rong et al. 1991, Camilo et al. 1996). However, the contribution of intestinal bacteria to whole body folate homeostasis in mammals is significantly less than the dietary source of folates (Visentin et al. 2014). 


\subsubsection{Folate absorption}

Dietary folate absorption primarily occurs in the proximal intestine within an acidic microenvironment (Fig. 1.2.) (Sid et al. 2017). The reduced folate carrier (RFC/SLC19A1), the proton-coupled folate transporter (PCFT/SLC46A1) and the folate receptors (FR $\alpha$ and FR $\beta$ ) are highly expressed along the apical membrane of enterocytes (Zhao et al. 2011, Visentin et al. 2014). Folate uptake is mainly mediated by PCFT, which optimally functions at an acidic pH in the small intestine (pH 4 to 6) (Qiu et al. 2006, Visentin et al. 2014). Natural dietary folates (polyglutamate form) are hydrolyzed to monoglutamates by glutamate carboxypeptidase II (in human intestine) or $\gamma$-glutamyl hydrolases (in rodent intestine) prior to absorption at the intestinal brush border membrane (Shafizadeh et al. 2007). Compared with folic acid, dietary folates tend to be more reduced and are commonly found as formyl and methyl polyglutamates (Perry 1971, Wright et al. 2007). Folic acid is a monoglutamate; therefore, hydrolysis is not necessary for intestinal absorption (Hu et al. 2016). Folic acid approaches $100 \%$ bioavailability and at least $85 \%$ of folic acid is bioavailable when consumed in the diet. However, only $50 \%$ of natural dietary folates are bioavailable (Institute of Medicine Standing Committee on the Scientific Evaluation of Dietary Reference et al. 1998, Iyer et al. 2009). Upon absorption by enterocytes, folic acid is reduced to dihydrofolate (DHF). Subsequently, DHF is further reduced to tetrahydrofolate (THF) that is the biological active form of folate (Wright et al. 2007, Bailey et al. 2009, Pietrzik et al. 2010). Although both reactions are catalyzed by DHF reductase, the initial enzymatic reduction of folic acid to DHF is the rate-limiting step (Bailey et al. 2009). The addition of one-carbon moiety to THF by serine hydroxymethyltransferase (SHMT) generates 5,10-methylene-tetrahydrofolate (5,10-methylene-THF). The 5,10-methylene-THF is further reduced to 5-methyl THF (5-MTHF) by 5,10-methylene-THF reductase 


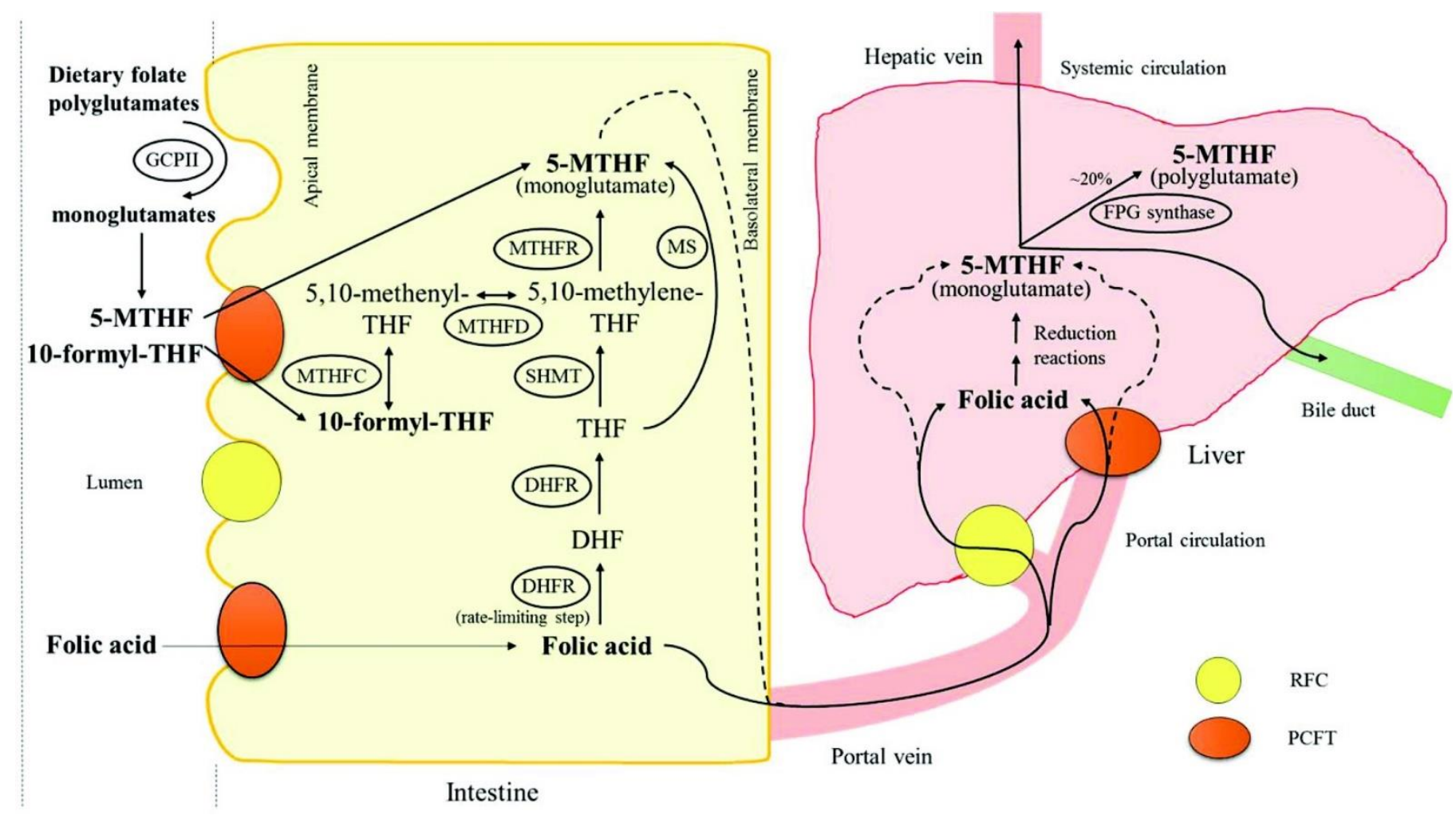

Figure 1.2. Folate absorption in the intestine and liver

The major routes of folate absorption and metabolism in the intestine and liver are illustrated. The key forms of folate that enters these organs are shown in bold. Unfilled ovals denote enzymes. DHF, dihydrofolate; THF, tetrahydrofolate; 5-MTHF, 5-methyltetrahydrofolate; DHFR, dihydrofolate reductase; SHMT, serine hydroxymethyltransferase; MTHFR, methylenetetrahydrofolate reductase; MTHFD, methylenetetrahydrofolate dehydrogenase; MTHFC, methylenetetrahydrofolate cyclohydrolase; MS, methionine synthase; GCPII, glutamate carboxypeptidase II; FPG synthase, folylpolyglutamate synthase; PCFT, protoncoupled folate transporter; RFC, reduced folate transporter. (Image based on Sid et al. 2017. Can J Physiol Pharmacol) 
(Tibbetts et al. 2010, Stover et al. 2011). The 5-MTHF is the main form of folate that is transported across the basolateral membrane of enterocytes and enters the portal circulation (Wright et al. 2007, Pietrzik et al. 2010). Because DHF reductase has a low capacity for reduction of folic acid in enterocytes, unmetabolized folic acid may be present in the portal circulation (Hu et al. 2016). High intakes of folic acid may also lead to accumulation of its unmetabolized form in the portal vein (Raghunathan et al. 1997, Pietrzik et al. 2010). The 5MTHF and folic acid derived from the gut is subsequently delivered to the liver via the portal vein (Wright et al. 2007, Pietrzik et al. 2010, Hu et al. 2016). The RFC and PCFT are widely expressed on the basolateral membrane of hepatocytes (Zhao et al. 2009, Zhao et al. 2011). However, previous studies suggest that the PCFT plays a major role in mediating folic acid and 5-MTHF transport to the liver (Horne et al. 1993, Hu et al. 2016). Once inside the cell, folic acid is metabolized to 5-MTHF, which undergoes polyglutamation by folylpolyglutamate synthase (Zhao et al. 2009, Visentin et al. 2014). Folate polyglutamates are efficiently retained by hepatocytes relative to their monoglutamate forms and are preferential substrates for folatedependent enzymatic reactions (Blom et al. 2006, Zhao et al. 2009). While up to $20 \%$ of the 5MTHF is retained by the liver, the remainder is delivered to extra-hepatic tissues by the systemic circulation or secreted into bile via the bile duct (Steinberg et al. 1979). Bile folates may be reabsorbed by the intestine and are subsequently distributed to the liver and other tissues (Steinberg et al. 1979, Zhao et al. 2009). Because the liver is a major organ for folate storage and metabolism (Wright et al. 2007), it plays an important role in maintaining whole body folate homeostasis (Steinberg et al. 1979). 


\subsubsection{Folate metabolism and biological function}

Folate mediated one-carbon transfer reactions are differentially distributed within the cytoplasm, nucleus, and mitochondria in mammalian cells (Figure 1.3) (Shin et al. 1976, Tibbetts et al. 2010, Stover et al. 2011, Sid et al. 2017). Intracellular one-carbon transfers are mediated by coenzymatic forms of THF, which carries one-carbon moieties (derived from histidine, serine, glycine, and formate) for amino acid, nucleotide biosynthesis, and methylation reactions (Blom et al. 2006, Tibbetts et al. 2010, Stover et al. 2011). In the cytoplasm and nucleus, 5,10methylene-THF serves as a substrate for biosynthesis of deoxythymidylate from deoxyuridylate, as well as for interconversion of glycine and serine (Herbig et al. 2002, Tibbetts et al. 2010). Serine and glycine biosynthesis may also occur in the mitochondria. In contrast, de novo purine nucleotide biosynthesis and remethylation of homocysteine to methionine are folate-dependent reactions that take place in the cytoplasm (Tibbetts et al. 2010, Stover et al. 2011). The 5,10methylene-THF is reversibly converted to 5,10-methenyl-THF by methylenetetrahydrofolate dehydrogenase (Herbig et al. 2002). The 5,10-methenyl-THF is a precursor for 10-formyltetrahydrofolate (10-formyl-THF) synthesis. The 10-formyl-THF provides one-carbon moieties for purine nucleotide synthesis, while 5-MTHF donates carbon units for methionine synthesis (Tibbetts et al. 2010). Nucleotide biosynthesis is required for RNA and DNA synthesis, and plays a key role in cell growth and proliferation. Remethylation of homocysteine to methionine also prevents homocysteine accumulation. Methionine is an essential precursor for the synthesis of $S$-adenosylmethionine (SAM), a principal methyl donor in cells. This cofactor regulates a number of fundamental cellular processes involved in cell signaling, protein localization, degradation of molecules, as well as gene transcription and translation (Miranda et al. 2007). Folate deficiency attenuates methionine synthesis from homocysteine, inhibits generation of 
SAM from methionine, and promotes accumulation of $S$-adenosylhomocysteine (SAH), a potent inhibitor of SAM-dependent methylation reactions (Stover 2004). Folate deficiency compromises one-carbon metabolism and is implicated in diseases such as hyperhomocysteinemia, alcoholic fatty liver disease, and NAFLD (Stover 2004, Christensen et al. 2010, Medici et al. 2013). One-carbon metabolism in the mitochondria is important for the synthesis of formate, which is a major source of carbon units for one-carbon metabolism that occurs in the cytoplasm (Barlowe et al. 1988, Tibbetts et al. 2010). The 10-formyl-THF serves as a carbon donor for formate synthesis and donates a formyl group to the methionine-tRNA (formylmethionine-tRNA), which is required for initiation of mitochondrial protein synthesis (Pike et al. 2010, Tibbetts et al. 2010). Taken together, folate-dependent one-carbon transfer reactions are vital for regulation of various intracellular metabolic processes (Zhao et al. 2009, Stover et al. 2011).

\subsubsection{Folate transporters}

The RFC, PCFT and folate receptors (FR $\alpha$ and FR $\beta$ ) are responsible for mediating transport of folates across the epithelium and into systemic tissues (Zhao et al. 2009, Visentin et al. 2014). While RFC is ubiquitously expressed in most tissues, highest expression of PCFT is detected in small intestine, kidney, liver, placenta, retina and brain (Zhao et al. 2009). Both RFC and PCFT are comprised of 12 transmembrane domains with the amino and carboxyl terminus directed in the cytoplasm (Visentin et al. 2014). The RFC normally functions at a neutral $\mathrm{pH}(\mathrm{pH}$ 7.4) and has a high affinity for transport of reduced folates (5-MTHF, 5-formylTHF) compared to folic acid (Zhao et al. 2009). This folate transporter is abundantly expressed in most tissues and mainly responsible for folate transport under physiological conditions (Wang et al. 2001). The 


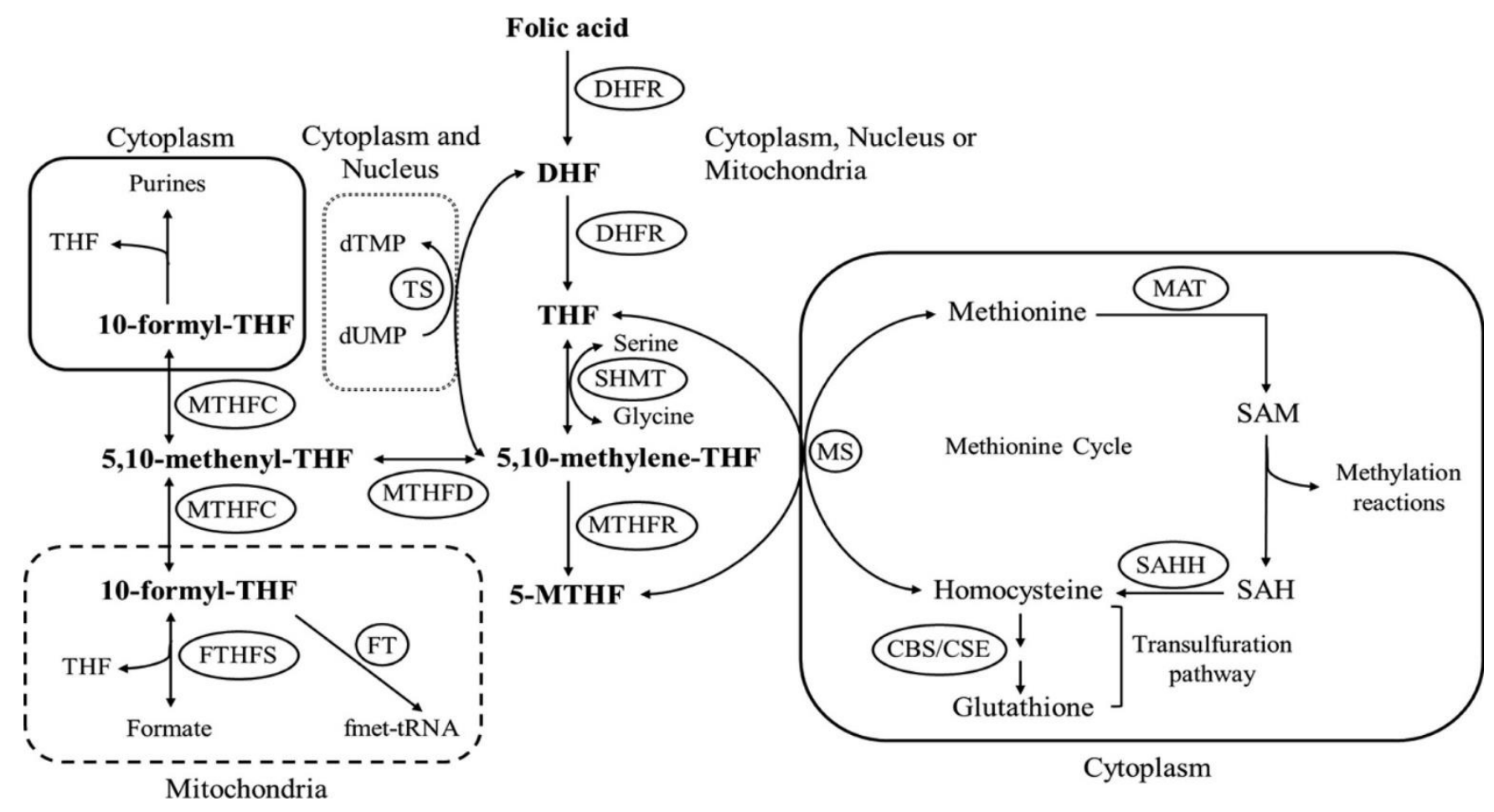

Figure 1.3. Compartmentalization of folate-dependent one-carbon metabolism

One-carbon metabolism for nucleotide biosynthesis, amino acid metabolism, and methylation reactions are distributed within intracellular compartments such as the cytoplasm, nucleus, and mitochondria. The boxes with solid lines denote reactions that only occur in the cytoplasm, the box with dashed lines denotes reactions that mainly occur in the mitochondria, and the box with dotted lines denotes reactions that occur in both the cytoplasm and nucleus. The other onecarbon metabolism reactions may occur in all 3 cellular compartments. Ovals denote enzymes. DHF, dihydrofolate; THF, tetrahydrofolate; 5-MTHF, 5-methyltetrahydrofolate; DHFR, dihydrofolate reductase; SHMT, serine hydroxymethyltransferase; MTHFR, methylenetetrahydrofolate reductase; MTHFD, methylenetetrahydrofolate dehydrogenase; MTHFC, methylenetetrahydrofolate cyclohydrolase; FTHFS, formyltetrahydrofolate synthetase; TS, thymidylate synthase; dTMP, deoxythymidylate; dUMP, deoxyuridylate; MS, methionine synthase; MAT, methionine adenosyltransferase; CBS, cystathionine- $\beta$-synthase; CSE, cystathionine-Y-lyase; SAM, $S$-adenosylmethionine; SAH, $S$-adenosylhomocysteine; SAHH, $S$ adenosylhomocysteine hydrolase; fmet-tRNA, formylmethionine-tRNA; FT, formyltransferase. (Image based on Sid et al. 2017. Can J Physiol Pharmacol) 
RFC is an antiporter and its function depends on transmembrane organic phosphate gradient that provides energy for transport of folates into cells (Visentin et al. 2012). In contrast, the PCFT exhibits a high affinity for transport of folic acid and reduced folates at a low $\mathrm{pH}$ optimum $(\mathrm{pH}$ 5.5). The PCFT is a symporter that relies on the proton gradient for transport of folates into cells (Qiu et al. 2006, Visentin et al. 2014). Human PCFT possesses two glycosylation sites, but they are not essential for transport function (Unal et al. 2008). On the other hand, FR $\alpha$ and FR $\beta$ are high-affinity folate binding proteins that are anchored to the cell membrane by glycosylphosphoinositol. While FR $\alpha$ is present in epithelial cells of the kidney, choroid plexus, retina, uterus and placenta, FR $\beta$ is mainly expressed in placenta, spleen, thymus and in CD $34^{+}$ monocytes (Zhao et al. 2009). These proteins mediate folate transport into cells via receptormediated endocytosis at a neutral to slightly acidic pH (Kamen et al. 2004, Salazar et al. 2007). Although folate binding proteins can transport folate into cells, they play a minor role compared to RFC and PCFT. The PCFT is mainly responsible for dietary folate absorption at the intestine, while both RFC and PCFT efficiently transport folate to peripheral tissues (Zhao et al. 2009).

Several transcriptional factors have been identified to regulate gene expression of RFC and PCFT (Matherly et al. 2007, Visentin et al. 2014). It was reported that the basic leucine Zipper Domain (bZip) containing proteins (eg. cJun/cFos and Creb1/ATF1), specificity proteins (eg. Sp1 and Sp3), upstream stimulatory factor (USF) and Ikaros-related protein exhibit binding sites on the RFC promoter. Post-translational modifications of these transcriptional factors may affect RFC expression (Matherly et al. 2007). Moreover, histone deacetylation and methylation at the RFC promoter was also shown to alter RFC expression (Worm et al. 2001, Liu et al. 2004). On the other hand, it was demonstrated that vitamin D3, nuclear respiratory factor-1 (NRF-1), and Kruppel-like factor 4 (KLF4) are involved in the regulation of PCFT expression (Eloranta et al. 
2009, Gonen et al. 2010, Furumiya et al. 2013). Post-translational modifications at the PCFT promoter may also alter its gene expression. Hypermethylation of the PCFT promoter was shown to impair PCFT expression and function (Diop-Bove et al. 2009). However, further studies are required to elucidate the molecular mechanisms involved in regulation of folate transporters.

\subsubsection{Regulation of folate homeostasis}

Since folate transporters are crucial for absorption of folates across the cell epithelial membrane, expression of folate transporters is important to ensure sufficient folate stores for folate dependent processes in the body (Zhao et al. 2009). Genetic deletion of mouse RFC gene is embryonic lethal due to failure of erythropoiesis in bone marrow, liver and spleen (Zhao et al. 2001). Similarly, loss of both alleles encoding mouse folate receptors leads to defects in early embryonic development (Piedrahita et al. 1999). Moreover, the PCFT-null phenotype is associated with hereditary folate malabsorption in humans (Qiu et al. 2006).

Folate homeostasis in the body is not only dependent on regulation of folate transport at the intestinal level, but is also affected by reabsorption of bile folates to the liver via the enterohepatic circulation (Steinberg et al. 1979). Because the liver is a major organ for folate storage and metabolism (Wright et al.2007), it has an important role in maintaining whole body folate homeostasis (Steinberg et al. 1979). Inadequate or excessive circulating folates may have adverse effects on human health (Hu et al. 2016). One study demonstrated that high intake of folic acid is associated with increased risk of recurring colorectal adenoma in patients (Cole et al. 2007). Another study reported that folic acid supplementation was not significantly associated with recurrent adenoma (Wu et al. 2009). Further studies are required to determine whether excessive folate intake may increase the risk of multiple recurring adenomas. Another concern is 
that high folic acid intakes may mask vitamin B12 deficiency and impair cognitive function (Morris et al. 2010). Therefore, proper clinical trials are warranted to determine the optimal dose of folic acid supplementation to prevent adverse effects.

\subsubsection{Implication of folate deficiency in disease}

Mandatory folic acid fortification of flour and grain was established in 1998 for prevention of birth defects in Canada and the United States (CDC 2010). General populations in these countries have achieved adequate folate intakes since implementation of the folic acid fortification policy (Bailey et al. 2010). However, folate deficiency may occur in countries without mandatory folic acid fortification policies (Garcia-Casal et al. 2005, Dhonukshe-Rutten et al. 2009). Folate deficiency may be attributed to perturbed folate transport that contributes to various pathophysiological states (Zhao et al. 2009). Folate absorption is impaired in individuals with chronic alcoholism and malabsorption disorders (Lucock 2000, Medici et al. 2013). Hereditary folate malabsorption due to mutations in the PCFT gene abolishes folate intestinal absorption and transport into the central nervous system, leading to folate deficiency (Qiu et al. 2006). Folate deficiency leads to anemia and the development of neurological disorders in newborns (Stover 2004, De Wals et al. 2007, Cario et al. 2011). Dihydrofolate reductase (DHFR) deficiency can compromise folate status in red blood cells and cause megaloblastic anemia, a condition of large, abnormal red blood cells (Lucock 2000, Cario et al. 2011). A low level of serum folates is frequently observed in patients with alcoholic fatty liver disease (Medici et al. 2013) and hyperhomocysteinemia. Hyperhomocysteinemia is an independent predictor of cardiovascular disease (Clarke 2000, Wald et al. 2002). Methylenetetrahydrofolate reductase deficiency is a common hereditary disorder of folate metabolism that causes severe 
hyperhomocysteinemia (Brattstrom et al. 1998, Burda et al. 2015). Although folic acid supplementation could effectively reduce homocysteine levels in the circulation (Boushey et al. 1995, Lonn et al. 2006), cardiovascular risk was not improved in patients with vascular disease (Lonn et al. 2006). Because folate is a crucial player in one-carbon metabolism, its deficiency has been associated with dysregulation of intracellular metabolic processes (Christensen et al. 2010, da Silva et al. 2014). Emerging evidence suggests that low circulating folate levels may be associated with the development of metabolic disorders including obesity and NAFLD (Hirsch et al. 2005, Mahabir et al. 2008).

\subsubsection{Role of folate in lipid and carbohydrate metabolism}

A number of studies have suggested that folate deficiency may contribute to the development of steatosis in rodents (Christensen et al. 2010, da Silva et al. 2014). Depletion of dietary folates in rodents was associated with high expression of lipid biosynthetic genes, which perturbs lipid metabolism in the liver (Champier et al. 2012). Moreover, hepatic lipid transport by VLDL was impaired in folate-deficient mice (Kim et al. 1994, Christensen et al. 2010, da Silva et al. 2014). Phosphatidylcholine (PC) is essential for VLDL assembly in hepatocytes (Fast et al. 1995, Zhao et al. 2009). The synthesis of PC from phosphatidylethanolamine (PE) by phosphatidylethanolamine methyltransferase (PEMT) was reduced during folate deficiency (Li et al. 2008, Christensen et al. 2010). A reduction in the PC to PE ratio may compromise lipid export by VLDL, and promote hepatic lipid accumulation (Christensen et al. 2010). In addition to lipoprotein assembly, PC is a major phospholipid required for bile secretion by the liver (Noga et al. 2003). Bile production, which is essential for absorption and digestion of dietary fats, was also decreased in folate-deficient rodents. Inhibition of bile production may have adverse effects 
on lipid metabolism (Sehayek et al. 2003, McNeil et al. 2008). On the other hand, hepatic insulin resistance can perturb the regulation of lipid metabolism (Fabbrini et al. 2010, Birkenfeld et al. 2014), and is a common feature observed in human and rodent models of NAFLD (Utzschneider et al. 2006, Larter et al. 2008). The regulation of glucose and lipid production is disrupted when insulin action is compromised in the liver (Saltiel et al. 2001, Birkenfeld et al. 2014). Abnormal lipid and carbohydrate metabolism in the liver is often associated with dysregulation of AMPactivated protein kinase (AMPK). The AMPK is a key regulator of metabolism in correspondence with energy balance (Hardie et al. 2012). Inactivation of AMPK was previously associated with hepatic lipid accumulation, hyperglycemia, and hyperinsulinemia in animal models with high-fat diet-induced NAFLD (Pu et al. 2012). The ability of folate to modulate lipid metabolism might involve regulation of AMPK. In summary, the hepatoprotective effect of folate may be attributed to its important role in metabolic regulation.

\subsubsection{Role of folate in oxidative stress and inflammation}

Oxidative stress is a complex, biological phenomenon that reflects an imbalance between the production of reactive oxygen species (ROS) and the antioxidant defense mechanisms (Halliwell et al. 2004, Kaludercic et al. 2014). This imbalance is clearly demonstrated in patients with NAFLD, who exhibit elevated levels of ROS and hepatic lipid peroxides (Seki et al. 2002, Videla et al. 2004) compromised hepatic and systemic antioxidant defenses (Loguercio et al. 2004, Hardwick et al. 2010). Hepatic oxidative stress is evident throughout the spectrum of NAFLD, and is a key mediator of hepatocellular injury (Seki et al. 2002, Rolo et al. 2012). Folic acid, as well as naturally occurring folates, have been suggested to have antioxidant functions due to their ability to directly scavenge ROS (Joshi et al. 2001, Gliszczynska-Swiglo et al. 2007). 
Dietary folate depletion in rodents was shown to significantly increase hepatic lipid peroxidation, and impair the activities of various antioxidant enzymes (Henning et al. 1997, Huang et al. 2001). Low folate status may disrupt mitochondrial redox homeostasis, and stimulate oxidative damage in rodent liver (Sawyer et al. 1999, Chou et al. 2007). Our previous studies demonstrated that folic acid could confer protective effects against oxidative stress in the liver (Woo et al. 2006, Sarna et al. 2012) and kidney (Hwang et al. 2011) in rodents. Folic acid supplementation effectively inhibited NADPH oxidase-mediated superoxide production, and restored the antioxidant response in hyperhomocysteinemic rats, and in high-fat diet-induced obese mice (Woo et al. 2006, Hwang et al. 2011, Sarna et al. 2012). Moreover, intracellular folate metabolism significantly contributes to the generation of NADPH, a source of reducing power for defense against oxidative stress. Depletion in folate metabolic enzymes was associated with low levels of NADPH and a reduction in the ratio of reduced glutathione to oxidized glutathione, which is an indicator of oxidative stress (Fan et al. 2014). Hepatic inflammation is another important pathological mediator of NAFLD (Day 2006). Oxidative stress may contribute to the progression of NAFLD by triggering the hepatic immune response. The by-products of lipid peroxidation (i.e. malondialdehyde and 4-hydroxynonenal) may activate liver immune cells and promote inflammation (Sutti et al. 2014). In NAFLD, chronic stimulation of the hepatic inflammatory response may enhance liver susceptibility to tissue injury, fibrosis, and cirrhosis (Elsharkawy et al. 2007, Sutti et al. 2014). The degree of inflammation in the liver is exacerbated with increased severity of NAFLD (Kleiner et al. 2005). Patients with NAFLD have high levels of pro-inflammatory cytokines, which are secreted by hepatocytes and immune cells (Day 2006). Folic acid supplementation in overweight and hyperhomocysteinemic patients was associated with reduction in pro-inflammatory cytokine levels (Wang et al. 2005, Solini et al. 2006). In 
contrast, macrophages grown in a folate-depleted medium had high expression of pro-

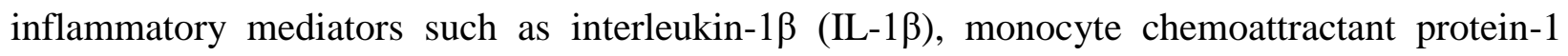
(MCP-1), interleukin-6 (IL-6) and tumor necrosis factor- $\alpha$ (TNF- $\alpha$ ) (Kolb et al. 2013). Macrophage-mediated release of inflammatory cytokines may contribute to the development of vascular disease (Ito et al. 2003, McLaren et al. 2011). We have observed that treatment of macrophages with folic acid attenuated homocysteine-induced pro-inflammatory cytokine expression (Au-Yeung et al. 2006). The ability of folic acid to reduce inflammatory cytokine levels further suggests that it may attenuate the inflammatory response that is associated with the development of cardiovascular disease (Au-Yeung et al. 2006, Kolb et al. 2013). Taken together, the sum of available evidence suggests that folic acid may exhibit both antioxidant and antiinflammatory functions.

\subsubsection{Role of folate in NAFLD}

Obese and overweight patients had significantly lower serum folate levels compared with normal weight individuals. Serum folate levels were decreased by $1.7 \%$ in correspondence to each unit increment of BMI in obese patients (Mahabir et al. 2008). A study based on the US National Health and Nutrition Examination Survey (NHANES, 2003-2006) reported that serum folate levels were significantly reduced in obese patients $(12.4 \mathrm{~g} / \mathrm{L} ; \mathrm{n}=1141)$ compared with normalweight individuals (13.1 g/L; n=1236). The regression analysis indicated an inverse relationship between serum folate and BMI regardless of adjustment for vitamin intakes and demographic variables such as gender, age, ethnicity, smoking status, and alcohol use. Low levels of serum folates were directly associated with decreased intakes of the vitamin in obese patients (Bird et al. 2015). Another study performed on childbearing age women before and after the USA folic 
acid fortification program (data obtained from NHANES III 1988-1994; n=5018 and from NHANES 1999-2000; $n=1351$ ) revealed that even after controlling for folate intake in food and supplements, a high BMI was associated with low serum folate levels. It was estimated that women with a BMI greater or equal to $30 \mathrm{~kg} / \mathrm{m} 2$ had to take an additional $350 \mathrm{~g} /$ day of folate to achieve similar serum folate levels as women in the lowest BMI category (less than $20 \mathrm{~kg} / \mathrm{m} 2$ ). The significant decrease of serum folates suggests that obese patients may be at risk for folate deficiency despite dietary folic acid fortification (Mojtabai 2004). In contrast, folate status was significantly improved in morbidly obese patients after gastric bypass surgery (Updegraff et al. 1981). Because treatment of obesity was able to restore folate levels in patients, this suggests that obesity may be the underlying cause for the imbalance of endogenous folate levels. Low folate levels in obese patients were associated with increased NAFLD severity. It was reported that serum folate levels were significantly reduced in obese female patients with severe NAFLD (21 nmol/L; n=17) compared with obese women with normal liver morphology or minimal liver damage (27 nmol/L; $\mathrm{n}=26)$ (Hirsch et al. 2005). One limitation of this study is a small sample size (Hirsch et al. 2005). Circulating folate levels were also markedly lower in patients with type 2 diabetes. Such a reduction in blood folate levels was correlated with high levels of fasting blood glucose, and increased expression of hepatic genes involved in the development of diabetes (Nilsson et al. 2015). Folate depletion may also lead to epigenetic and transcriptional alterations in the liver, and contribute to the pathogenesis of type 2 diabetes (Nilsson et al. 2015). While the sum of clinical evidence suggests that low folate status may have an important implication in metabolic disease, a causal relationship cannot be established. In addition to the findings from human studies, animal-based studies also support the notion that folate homeostasis is impaired in obesity and diabetes. In the study by Lam et al., experiments were 
conducted in diabetic/obese $(d b / d b)$ and nondiabetic/lean $(m / d b)$ C57BL/KsJ mice. It was demonstrated that plasma folate concentrations in the $d b / d b$ mice $(29.62 \mu \mathrm{g} / \mathrm{L})$ were profoundly reduced compared with $m / d b$ mice $(45.22 \mu \mathrm{g} / \mathrm{L})$. A limitation of this study was that it was unclear whether blood was collected under fasting or fed conditions, which might affect the interpretation of the plasma folate readings (Lam et al. 2009). Overall, the reduction in serum folate levels observed in obese patients and in animal models suggests a potential interrelationship between perturbation of folate status and the development of NAFLD. In summary, current research evidence suggests a potential role of folate in NAFLD development ( Figure 1.4). 


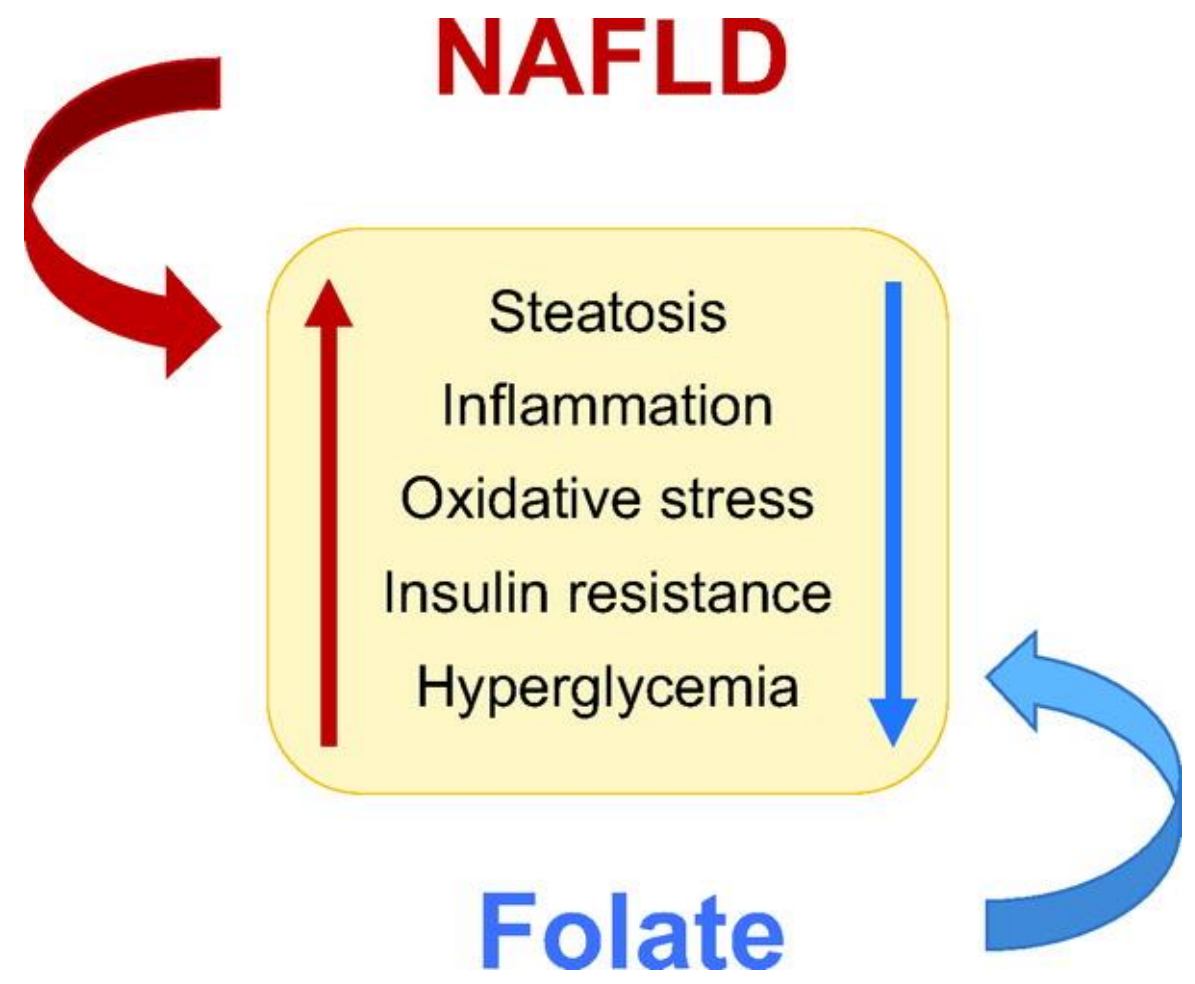

Figure 1.4. Potential role of folate in NAFLD

The relationship between folate and NAFLD is illustrated. NAFLD is characterized by steatosis, inflammation, oxidative stress, as well as insulin resistance and hyperglycemia. Folate supplementation in rodents reduces metabolic abnormalities associated with NAFLD (Image based on Sid et al. 2017. Can J Physiol Pharmacol) 


\section{HYPOTHESES AND OBJECTIVES}




\subsection{Rationale}

NAFLD is a major health burden worldwide due to the growing epidemic of obesity and diabetes (Loomba et al. 2013). As there is currently no pharmacological agent approved for the treatment of NAFLD, the world is in search of a safe and effective treatment avenue (Schuppan et al. 2013). Folate, a water-soluble B vitamin participates in one-carbon transfer reactions that are essential for cell metabolism (Stover 2004, Tibbetts et al. 2010). Dysregulation of folatedependent one-carbon metabolism has been implicated in NAFLD-related comorbidities such as obesity, type 2 diabetes, and the metabolic syndrome (Hirsch et al. 2005, Mahabir et al. 2008, da Silva et al. 2014, Nilsson et al. 2015). However, the hepatoprotective effect of folate in NAFLD is poorly understood. Understanding the role of folate in the context of fatty liver may position this vitamin as a potential therapeutic for NAFLD.

\subsection{Hypotheses}

Vitamins are essential micronutrients that are required for proper growth, health maintenance and body function. Certain vitamin deficiencies may perturb metabolic function and contribute to the development of NAFLD. We hypothesize that (1) regulation of folate homeostasis in the body is impaired during diet-induced NAFLD; (2) supplementation of folic acid is beneficial for management of NAFLD through improving hepatic lipid and glucose metabolism, as well as hepatic inflammation. 


\subsection{Objectives}

The general objective of my research was to investigate the role of folate in the context of highfat diet-induced NAFLD. The specific objectives are outlined as follows:

Objective 1: To identify the underlying mechanisms that is responsible for regulating folate status during high-fat diet feeding.

Objective 2: To investigate the effects of folic acid supplementation on AMPK-mediated metabolic regulation in high-fat diet fed mice

Objective 3: To investigate the regulation of hepatic inflammation by folic acid supplementation in high-fat diet fed mice 


\section{MANUSCRIPTS}




\section{STUDY 1 - MANUSCRIPT 1}

\subsection{High-fat diet consumption reduces hepatic folate transporter expression via nuclear respiratory factor-1}

V. Sid, Y.L. Siow, Y. Shang, C.W. Woo, K. O. J Mol Med. 96(11):1203-1213 (2018) 


\subsubsection{Abstract}

Folate is an essential micronutrient for biological function. The liver, a primary organ for folate metabolism and storage, plays an important role in folate homeostasis. Proton-coupled folate transporter (PCFT) and reduced folate carrier (RFC) are the major folate transporters responsible for folate uptake at basolateral membrane of hepatocytes. Low serum folate levels are frequently associated with obesity. We investigated the mechanism that regulated folate status in a mouse model with diet-induced obesity. Mice (C57BL/6J) were fed a high-fat diet (60\% kcals fat) for 8 weeks. Mice displayed increased hepatic lipid accumulation, and decreased folate levels in the liver and serum compared to mice fed a normal chow diet (10\% kcals fat). High-fat diet fed mice had low expression of PCFT and RFC, decreased nuclear respiratory factor-1 (NRF-1)/DNA binding activity. Treatment with NRF-1 siRNA or palmitic acid reduced folate transporter expression in hepatocytes. Inhibition of NRF-1 mediated folate transporter expression significantly reduced intracellular folate levels. These results suggest that chronic consumption of high-fat diets impairs folate transporter expression via NRF-1 dependent mechanism, leading to reduced hepatic folate storage. Understanding the regulation of folate homeostasis in obesity may have an important implication in current guideline of folate intake. 


\subsubsection{Introduction}

Folate is a water-soluble B vitamin obtained from diets. This micronutrient is essential for biological functions including sulfur-containing amino acid metabolism, nucleotide synthesis and methylation reactions (Zhao et al. 2009, Stover et al. 2011). Due to the implementation of mandatory folic acid fortification policy, dietary deficiency of folate is believed to be uncommon in generally healthy populations in Western countries (Bailey et al. 2010, CDC 2010). However, despite adequate intake of folate from diets and supplements, obese individuals and patients with non-alcoholic fatty liver disease (NAFLD) are reported to have low folate levels in the circulation (Mojtabai 2004, Mahabir et al. 2008, da Silva et al. 2013, Xia et al. 2018). In addition, low folate status is also observed in patients with malabsorption, impaired kidney function or liver disease, i.e. in vulnerable senior population (Klipstein et al. 1965, Leblanc et al. 2000, Qiu et al. 2006). Owing to the increased epidemic of obesity and prevalence of NAFLD in the recent decades (Loomba et al. 2013), folate deficiency is an emerging issue, especially for patients with obesity (da Silva et al. 2013, Sid et al. 2017).

Obesity is one of the most common risk factors for various chronic diseases (Must et al. 2000). It has been reported that low levels of serum folate are associated with enhanced NAFLD severity in obese patients (Hirsch et al. 2005, Xia et al. 2018). However, the mechanism that leads to low folate status in obese patients is not fully understood. Chronic consumption of high-fat diets is a major contributor to NAFLD and obesity (Golay et al. 1997, Angulo 2007). The high-fat diet fed rodents develop hepatic histopathological features such as lipid accumulation, oxidative stress and inflammation, which resemble NAFLD (Hebbard et al. 2011, Wu et al. 2013). In previous studies, we observed that folic acid supplementation reduced oxidative stress as well as improved hepatic lipid and glucose metabolism in mice fed a high-fat diet (Sarna et al. 2012, Sid et al. 
2015). However, it is not clear whether chronic consumption of high-fat diets affects folate homeostasis. The liver is a primary organ responsible for folate metabolism and storage as well as folate distribution to the circulation (Steinberg et al. 1979, Steinberg 1984, Wright et al. 2007). Mammals lack the enzymatic capacity for folate biosynthesis, and therefore, folate must be acquired from dietary sources to meet physiological requirement (Zhao et al. 2009). Following intestinal absorption, folate enters the hepatic portal circulation and is taken up by the liver via folate transporters (Wright et al. 2007, Zhao et al. 2011). The proton-coupled folate transporter (PCFT, encoded by SLC46A1 gene) and the reduced folate carrier (RFC, encoded by SLC19A1 gene) are transmembrane proteins that mediate folate uptake in hepatocytes. These two folate transporters are abundantly expressed at the basolateral membrane of hepatocytes (Zhao et al. 2009, Zhao et al. 2011). Regulation of folate transport is important to ensure adequate folate storage in the liver, and to maintain folate levels in the systemic and enterohepatic circulation (Steinberg 1984, Medici et al. 2013). The nuclear respiratory factor-1 (NRF-1) is recently identified as a key transcriptional factor that controls the expression of PCFT gene expression in the intestine (Gonen et al. 2010). However, the regulation of hepatic folate transporters in the context of obesity is unclear.

In the present study, we evaluated the impact of chronic consumption of a high-fat diet on hepatic folate transporter expression and folate status in mice, and the mechanisms involved. Mice fed a high-fat diet for 8 weeks displayed hepatic lipid accumulation and a significant decrease in folate transporter (PCFT, RFC) expression in the liver. We investigated the mechanisms by which high-fat diet reduced hepatic folate transporter expression. We demonstrated that downregulation of NRF-1 led to reduced expression of folate transporters in the liver of mice fed a high-fat diet. 


\subsubsection{Material and Methods}

i) Animal model

Male C57Bl/6J mice aged 6 weeks were purchased from Central Animal Care Services (University of Manitoba, Winnipeg, MB, CAN) and were isolated for 1 week quarantine before the beginning of the experimental period. Mice were fed a normal chow diet (D12450B) or a high-fat diet (D12492) for 8 weeks. The normal chow diet consisted of $10 \% \mathrm{kcal}$ fat, $20 \% \mathrm{kcal}$ protein, and $70 \% \mathrm{kcal}$ carbohydrate with $2 \mathrm{mg}$ of folic acid/ $\mathrm{kg}$ of diet, while the high-fat diet consisted of $60 \% \mathrm{kcal}$ fat, $20 \% \mathrm{kcal}$ protein, and $20 \% \mathrm{kcal}$ carbohydrate with $2.6 \mathrm{mg}$ of folic $\mathrm{acid} / \mathrm{kg}$ of diet. All the purified diets were formulated and prepared by Research Diets Inc. (New Brunswick, NJ, USA). Mice were kept on a $12 \mathrm{~h}$ light/12h dark cycle with access to food and water ad libitum. Mice were sacrificed after 8 weeks and blood was collected for serum preparation. Animal body weights were recorded prior to feeding and at the end of the experimental feeding period. Following euthanasia, tissues were collected and immediately placed in liquid nitrogen, then kept frozen at $-80^{\circ} \mathrm{C}$ until further analysis. All procedures were performed in accordance with the Guide to the Care and Use of Experimental Animals published by the Canadian Council on Animal Care and approved by the University of Manitoba Protocol Management and Review Committee.

ii) Liver histology and lipid analysis

A portion of the liver tissue was soaked in $10 \%$ neutral-buffered formalin overnight and embedded in paraffin. Paraffin-embedded cross sections were prepared on glass slides. Liver sections were deparaffinized and stained with hematoxylin and eosin (H\&E) staining to examine histological changes of the liver (Woo et al. 2005). Images of H\&E sections were captured using 
an Olympus BX43 light microscope equipped with a Q-Color3 camera and were analyzed at 100x and 200x magnification. Lipids in the liver tissue were extracted according to the Folch method (Folch et al. 1957). The total lipid levels in the liver were measured using the sulfophospho-vallin colorimetric reaction, according to manufacturer's instructions (BQ Kits, San Diego, CA, USA).

\section{iii) Cell culture}

HepG2 cells (human hepatoblastoma cell line HB8065; American Type Culture Collection, Manassas, VA, USA) were cultured in DMEM (VWR, Mississauga, ON, CAN) supplemented with $10 \% \mathrm{FBS}$, in a humidified atmosphere at $37^{\circ} \mathrm{C}$ with $5 \% \mathrm{CO}_{2}$. Cells were plated at a density of $5 \times 10^{5}$ and treated with or without palmitic acid $(0.3 \mathrm{mM})$. It was previously reported that plasma concentrations of palmitic acid in patients ranged from 0.1 to $0.3 \mathrm{mM}$ (Fraser et al. 1999). Other studies showed that treatment of $\mathrm{HepG} 2$ cells with $0.3 \mathrm{mM}$ palmitic acid caused significant changes in lipid metabolism and cytokine expression (Joshi-Barve et al. 2007, Wu et al. 2013, Sid et al. 2015). Palmitic acid (PA; Sigma Aldrich, Oakville, ON, CAN) was dissolved in $10 \%$ BSA (essentially fatty acid free, Sigma Aldrich, Oakville, ON, CAN) and 5\% ethanol with light shaking overnight at $37^{\circ} \mathrm{C}$ (Wu et al. 2013, Sid et al. 2015).

iv) Cell viability

Cell viability was determined by a colorimetric assay with 3-(4,5-dimethylthiazol-2-Yl)-2,5diphenyltetrazolium bromide (MTT). In brief, cells were plated in a 96 well plate a density of 1.0 $\times 10^{4}$ cells per well. After incubation with palmitic acid $(0.1-0.3 \mathrm{mM})$ for 12 hours, cells were 
incubated for 4 hours in the presence of MTT $(2 \mathrm{mg} / \mathrm{mL})$. MTT-formazan was extracted by DMSO for 2 hours. The optical density of formazan $(540 \mathrm{~nm})$ was then detected.

\section{v) Real-time PCR analysis}

Total RNA was prepared from the liver tissue and cells using Trizol reagent (Thermo Fisher Scientific, Waltham, MA, USA) according to manufacturer's instructions. In brief, $2 \mu \mathrm{g}$ of RNA was converted to cDNA by reverse transcription. The mRNA levels of various genes were assessed using the iQ5 real-time PCR detection system (Bio-Rad, Mississauga, ON, CAN). The real-time PCR reaction mixture consisted of $2 \mu 1$ of cDNA product, $0.4 \mu \mathrm{M}$ of $5^{\prime}$ and 3' primers and iQ-SYBR green supermix reagent (Bio-Rad, Mississauga, ON, CAN). The cycle threshold (CT) values of the target genes were normalized against $\beta$-actin CT values. Relative mRNA levels were quantified according to the $\Delta \Delta \mathrm{Ct}$ method. The primer sequences of PCFT, RFC, Folr1, DHFR, ABCG2, Mrp3, NRF-1 and $\beta$-actin (Thermo Fisher Scientific, Waltham, MA, USA) are listed (Table 1).

vi) Western immunoblotting analysis

The protein levels of PCFT and RFC were determined by Western immunoblotting analysis. In brief, liver proteins $(10-60 \mu \mathrm{g})$ were separated by electrophoresis in an 8 or $10 \%$ SDS polyacrylamide gel, and proteins were transferred from the gel to a nitrocellulose membrane for 1h. Non-specific sites on the membrane were blocked with 5\% non-fat dry milk in $20 \mathrm{mM}$ Tris$\mathrm{Cl}, 150 \mathrm{mM} \mathrm{NaCl}, 0.1 \%$ Tween 20, $\mathrm{pH} 7.4$ (TBST) at room temperature for $1 \mathrm{~h}$, and incubated with primary antibody overnight at $4^{\circ} \mathrm{C}$. Primary antibodies were diluted in 5\% milk in TBST (1:1000). Primary antibodies used for mouse liver samples were: rabbit anti-HCP1 (PCFT) 
polyclonal antibody (ab25134, Abcam, Cambridge, MA, USA) and mouse anti-RFC-1 monoclonal antibody (sc-271276, Santa Cruz Biotechnology, Dallas, TX, USA). Primary antibodies used for human HepG2 cell lysates were: rabbit anti-HCP1 (PCFT) polyclonal antibody (NBP1-06603, Novus Biologicals, Littleton, CO, USA), rabbit anti-SLC19A1 (RFC) polyclonal antibody (ab62302, Abcam, Cambridge, MA, USA) and rabbit anti-NRF-1 polyclonal antibody (\#12381, Cell Signaling, Danvers, MA, USA). Secondary antibodies were diluted in 5\% milk in TBST (1:1000). Membranes were subsequently probed with secondary antibodies including HRP-conjugated anti-mouse IgG antibody (\#7076, Cell Signaling, Danvers, MA, USA) or anti-rabbit IgG antibody (\#7074, Cell Signaling, Danvers, MA, USA) for $1 \mathrm{~h}$ at room temperature, and visualized using the Luminata Crescendo chemiluminescent HRP detection reagent (Millipore (Canada) Ltd, Etobicoke, ON, CAN). To ensure equal protein loading, the same membranes were reprobed with rabbit anti- $\beta$-actin monoclonal antibody (1:2000; \#4967, Cell Signaling, Danvers, MA, USA).

vii) Electrophoretic mobility shift assay (EMSA)

Nuclear proteins were prepared from the liver tissue and HepG2 cells (Woo et al. 2005, Woo et al. 2006). DNA oligonucleotides were biotin-labelled according to the Biotin 3' End DNA labelling kit (Thermo Fisher Scientific, Waltham, MA, USA). The sense strand sequences containing the NRF-1 binding site used in the study were: 5'GCGCAGGCGCAGACAGCACAGACTGGT3' (mouse; GenBankTM accession number NM_026740) and 5'GACGCCGGGCGCAGGCGCAGACAGCG3' (human; GenBankTM accession number EU185738.1). The core consensus sequence of NRF-1 is underlined (Gonen et al. 2010). EMSA was performed using the LightShift Chemiluminescent EMSA Kit (Thermo 
Fisher Scientific, Waltham, MA, USA). The labeled oligonucleotides were added to a reaction mixture $(20 \mu \mathrm{l})$ that contained $2 \mu \mathrm{g}$ of nuclear extract according to manufacturer's instructions, with some minor modifications. Poly [d(A-T)] (50 ng per reaction) was used as a nonspecific DNA competitor. The NRF-1 binding site is a GC-rich sequence and use of poly [d(I-C)] can compete out the NRF-1 binding site. Reaction mixtures were incubated at room temperature for 20 minutes. The cold competition assay was performed by incubating nuclear extracts with $1000-$ fold excess of unlabelled oligonucleotides before addition of labelled probe. Following the incubation, reaction mixtures were loaded in a $6 \%$ nondenaturing polyacrylamide gel to allow for separation of DNA-protein complexes, and transferred to a nylon membrane for detection using the Chemiluminescent Nucleic acid Detection Module Kit (Thermo Fisher Scientific, Waltham, MA, USA). The supershift assay was performed by incubating liver nuclear proteins $(2 \mu \mathrm{g})$ with $2 \mu 1$ and $4 \mu 1$ of anti-NRF-1 antibody (\#12381, Cell Signaling, Danvers, MA, USA) for 20 minutes followed by nondenaturing polyacrylamide gel (4\%) electrophoresis.

viii) Cell transfection

HepG2 cells were transfected with NRF-1 siRNA duplex oligoribonucleotides (Stealth RNAiTM, Invitrogen, Carlsbad, CA, USA) according to the manufacturer's instructions. For a negative control, cells were transfected with StealthTM RNAi negative control (Invitrogen, Carlsbad, CA, USA) consisting of a scrambled sequence that was unable to inhibit gene expression. At $48 \mathrm{~h}$ after transfection, the cells were collected, and mRNA levels of NRF-1, PCFT and RFC were measured. Cells were also collected after 72h transfection, and protein and folate levels were measured. 
ix) Folate measurement

Total folate concentrations in the liver and serum, as well as in cultured hepatocytes, were determined using the L. rhamnosus microplate assay, as followed by manufacturer's instructions provided in the Folic acid Vitamin B9 Microbiological Test Kit (Alpco, Salem, NH, USA).

x) Statistical analysis

The results were analyzed by a two-tailed Student's $t$-test, followed by post hoc analysis using Newman-Keuls test. The data was presented as the means \pm S.E. A $p$ value less than 0.05 were considered statistically significant. 


\subsubsection{Results}

i) High-fat diet feeding reduced folate levels in the liver and serum High-fat diet feeding for 8 weeks caused a significant decrease in folate levels in the liver (Fig. 3.5A) and serum (Fig. 3.5B). Mice fed a high-fat diet exhibited a significant increase in body weight compared to those maintained on a control diet (Fig. 3.5C). There was a significant elevation of total lipid levels in the liver (Fig. 3.5D). Hepatic lipid accumulation was further examined by H\&E staining. Mice fed a high-fat diet had an increased accumulation of lipid vacuoles in the liver compared to the control mice (Fig. 3.5E).

ii) Decreased folate transporter expression in the liver of mice fed a high-fat diet and in fatty acid treated hepatocytes

The uptake of folate from the hepatic portal system to the liver is mediated via folate transporters. The mRNA expression of folate transporters (PCFT, RFC) was significantly decreased in the liver of mice fed a high-fat diet (Fig. 3.6A, C). In addition, folate receptor alpha (Folr1) mRNA expression was also significantly reduced in response to high-fat diet feeding (Fig. 3.6E). In accordance with these results, there was a marked reduction of PCFT and RFC protein in the liver of mice fed a high-fat diet (Fig. 3.6B, D). The ATP binding cassette subfamily $\mathrm{G}$ member 2 ( $\mathrm{ABCG} 2$ ) is the predominant $\mathrm{ABC}$ transporter that exports folate from the liver (Ahmad Najar et al. 2016). High-fat diet feeding did not affect mRNA expression of ABCG2 (Fig. 3.6F). The multidrug resistance-associated protein 3 (MRP3) is another ABC transporter that exports folate from the liver (Zhao et al. 2011). High-fat diet feeding significantly increased mRNA expression of MRP3 in the liver (Fig. 3.6G). Such upregulation of MRP3 expression might be an adaptive response to low folate levels in the circulation (Liu et al. 
2005). Palmitic acid is a major saturated fatty acid in the high-fat diet. Treatment of HepG2 cells with various doses of palmitic acid $(0.1-0.3 \mathrm{mM})$ did not significantly affect cell viability (Fig. 3.7). Incubation with palmitic acid resulted in a significant decrease in PCFT and RFC mRNA expression (Fig. 3.8A, C), as well as protein levels (Fig. 3.8B, D). Furthermore, incubation of cells with palmitic acid resulted in a significant reduction of intracellular folate concentrations (Fig. 3.8E). These results indicated that high-fat diet feeding or fatty acid treatment impaired hepatic folate transporter expression.

iii) Reduction of hepatic folate transporter expression was mediated by NRF-1

To determine whether the reduction of PCFT and RFC expression in the liver following chronic high-fat diet feeding was caused by changes in transcriptional regulation, EMSA was performed. The mouse and human PCFT gene contains NRF-1 binding sites in its promoter region (Fig. 3.9A, C) (Gonen et al. 2010). The NRF-1/DNA binding activity was significantly decreased in the liver of mice fed a high-fat diet compared to those fed a control diet (Fig. 3.9A). To identify the proteins in the NRF-1/DNA complex, supershift assay was performed using anti-NRF-1 antibodies. Pre-incubation of liver nuclear proteins with anti-NRF-1 antibodies resulted in a slower migration of the protein-DNA complex (Fig. 3.9B), indicating that NRF-1 was involved in DNA binding. In addition, palmitic acid treatment also significantly reduced DNA binding activity of NRF-1 in HepG2 cells (Fig. 3.9C). However, treatment with palmitic acid did not significantly change NRF-1 mRNA expression (Fig. 3.9D). To further confirm that NRF-1 was involved in the regulation of hepatic PCFT and RFC gene expression, hepatocytes were transiently transfected with either NRF-1 siRNA or scrambled siRNA (negative control). Transfection of cells with NRF-1 siRNA significantly abolished NRF-1 mRNA and protein 
expression (Fig. 3.10A, D), as well as inhibited the expression of PCFT and RFC (Fig. 3.10B, C, E, F). In contrast, scrambled siRNA transfection in hepatocytes had no effect on NRF-1, PCFT, and RFC gene and protein expression (Fig. 3.10A, B, C, D, E, F). There was no significant difference in cell viability between NRF-1 siRNA transfection and scrambled siRNA transfection (Fig. 3.11). Palmitic acid treatment did not cause further reduction of PCFT and RFC mRNA expression in siNRF-1 transfected cells (Fig. 3.12A, B). Inhibition of NRF-1 mediated folate transporter expression caused a significant decrease in intracellular folate concentrations (Fig. 3.10G). These results suggested that NRF-1 might play a role in regulating hepatic folate transporter expression and folate content. In addition to folate transporters, we also measured gene expression of dihydrofolate reductase (DHFR), the rate limiting enzyme that converts folate to its bioactive form (tetrahydrofolate). Results showed that following NRF-1 siRNA transfection in HepG2 cells, the mRNA expression of DHFR was significantly elevated (Fig. 3.10H). It was plausible that in response to low intracellular folate levels caused by decreased folate transporter expression, DHFR might have been upregulated as an adaptive reaction. 
A

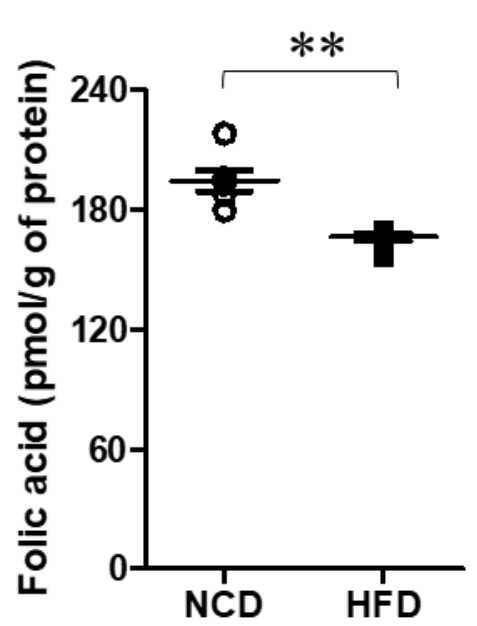

C

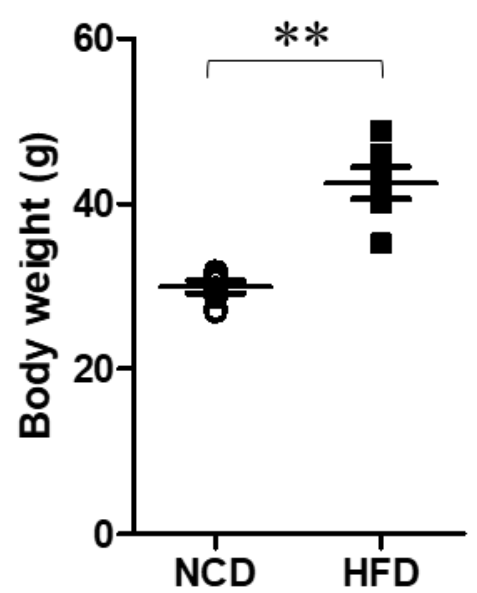

B
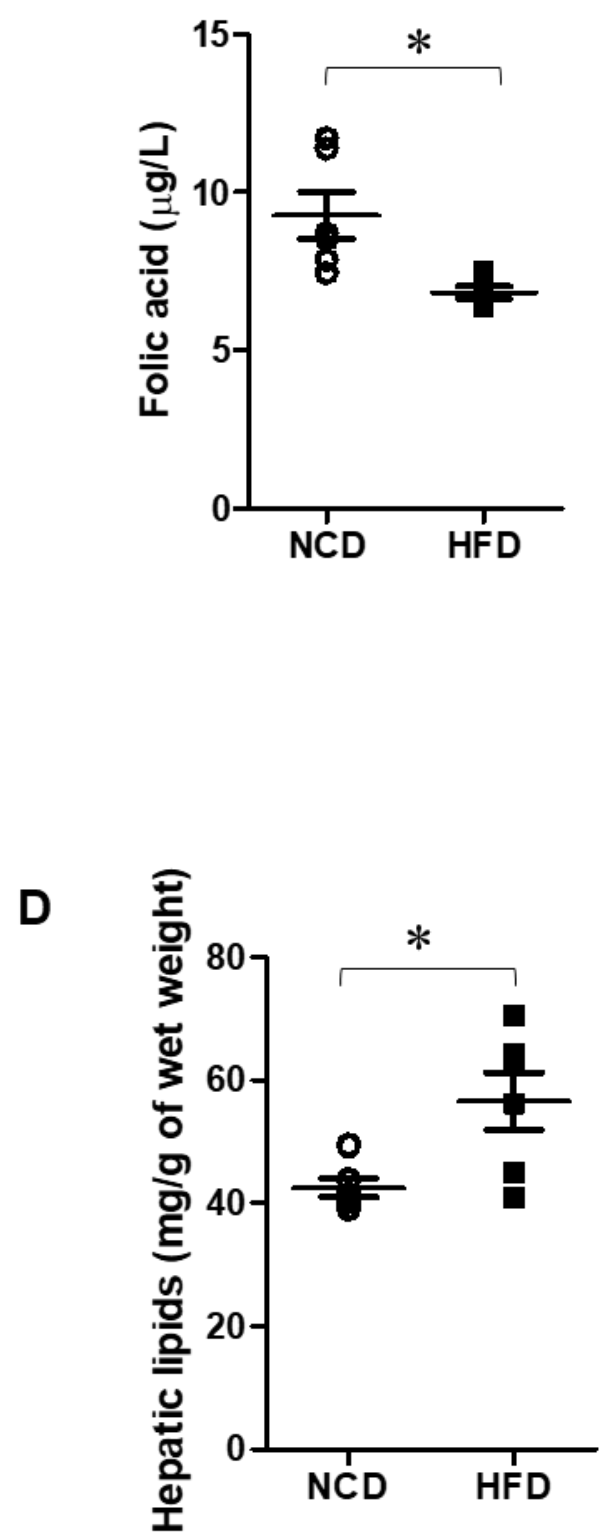
E

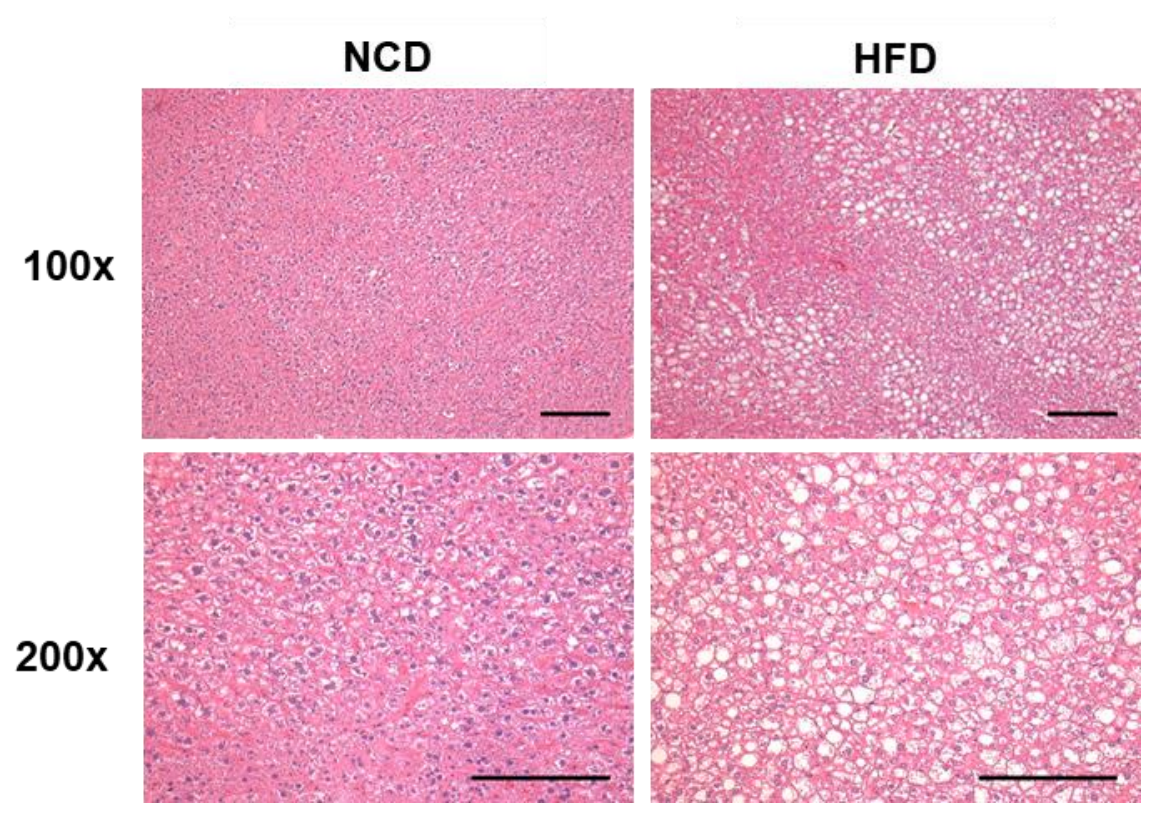

\section{Figure 3.5. Body weight, liver lipids and folate levels}

Mice were fed a normal chow diet (NCD) or a high-fat diet (HFD) for 8 weeks. (A) Liver folate and (B) serum folate levels were measured. (C) Body weight and (D) hepatic lipids were measured. (E) Representative H\&E staining images of liver sections are shown. The bar on the images represents $100 \mu \mathrm{m}$. Results are expressed as means \pm S.E. $(\mathrm{n}=6){ }^{*} p<0.05$ and $* * p<0.01$ when compared with the value obtained from the control group (NCD). 
A

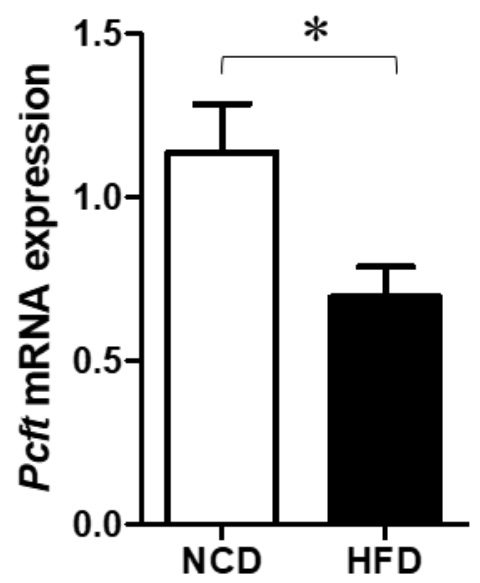

C

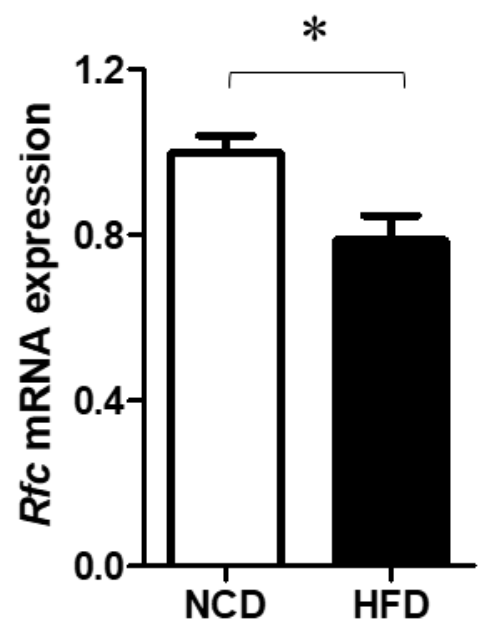

B
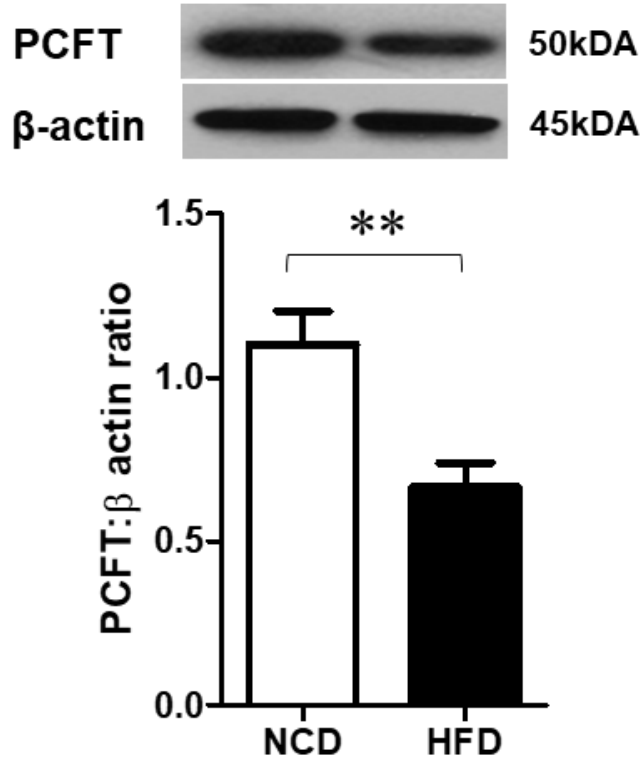

D

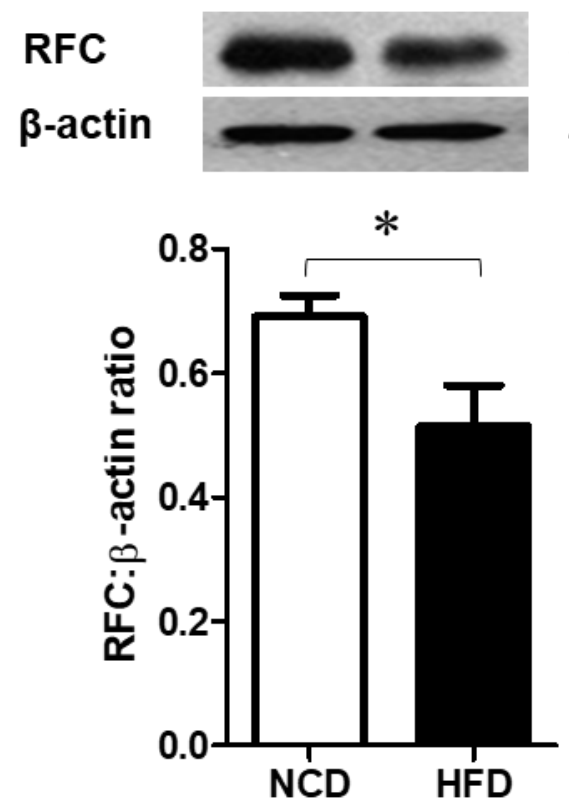


$\mathbf{E}$

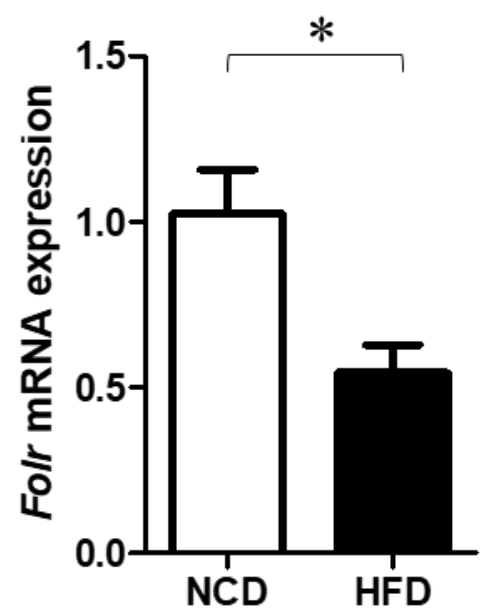

G

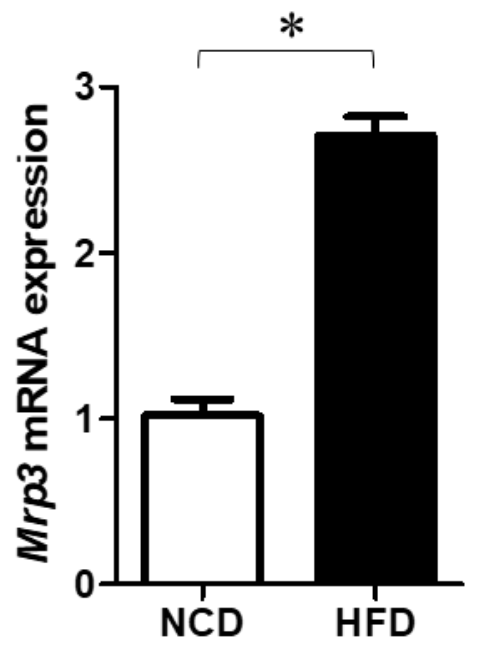

$\mathbf{F}$

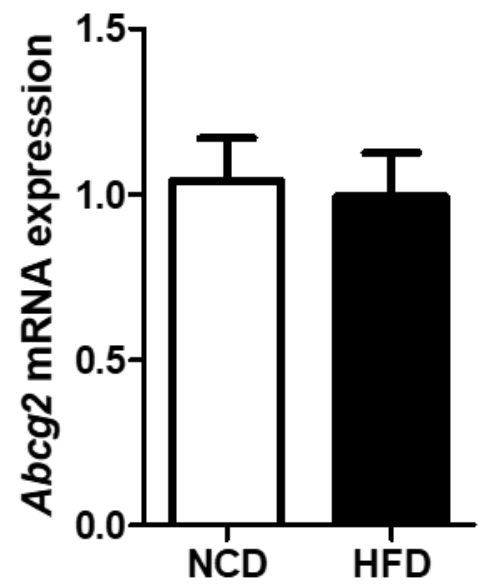

Figure 3.6. Folate transporter mRNA and protein expression in the liver

Mice were fed a normal chow diet (NCD) or a high-fat diet (HFD) for 8 weeks. (A) PCFT mRNA and (B) protein expression were measured. (C) RFC mRNA and (D) protein expression in liver tissue were measured. (E) Folr1, (F) ABCG2 and (G) MRP3 mRNA expression in liver tissue was measured. The mRNA levels were determined by real-time PCR analysis and the protein levels were determined by Western immunoblotting analysis. Results are expressed as means \pm S.E. $(\mathrm{n}=6) . * p<0.05$ and $* * p<0.01$ when compared with the value obtained from the control group (NCD). 


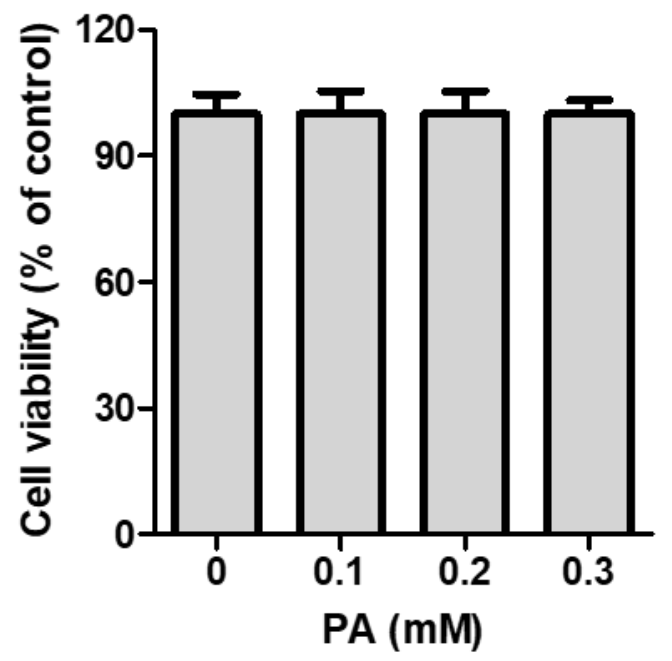

Figure 3.7. Effect of palmitic acid on cell viability

Cells were incubated with palmitic acid (PA, $0.1-0.3 \mathrm{mM})$ for 12 hours. Cell viability was assessed by incubating cells with 3-(4,5-dimethylthiazol-2-Yl)-2,5-diphenyltetrazolium bromide (MTT). The viability of cells without palmitic acid treatment was expressed as $100 \%$. Results are expressed as means \pm S.E. $(n=6)$. 
A

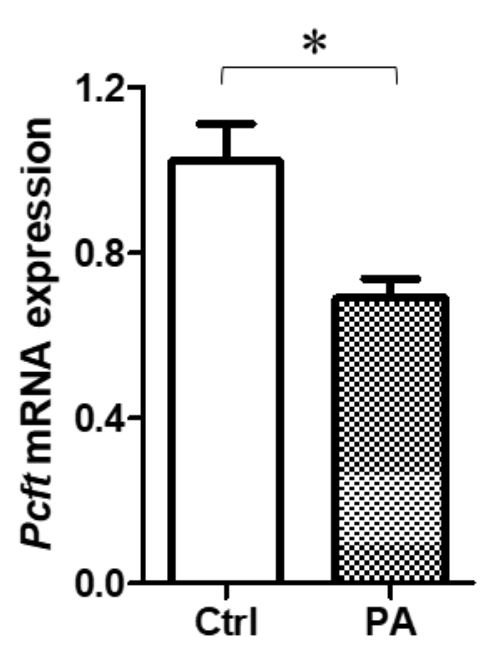

C

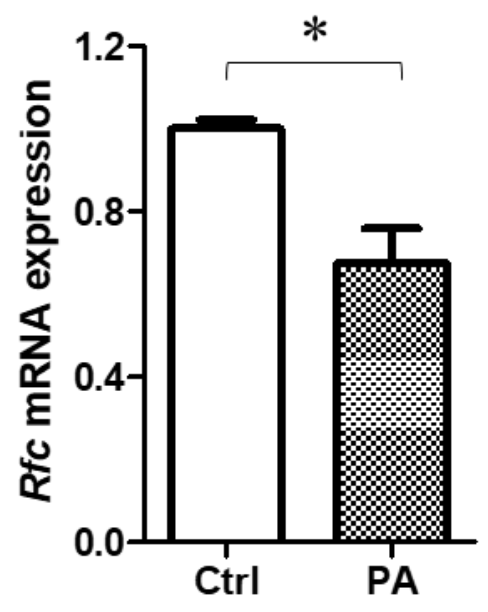

E

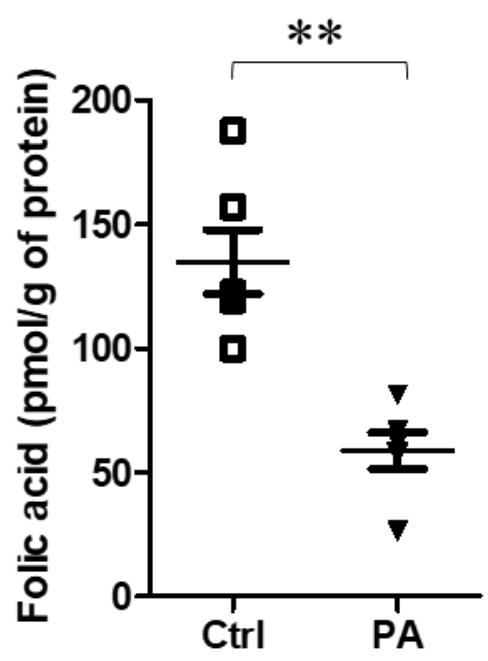

B
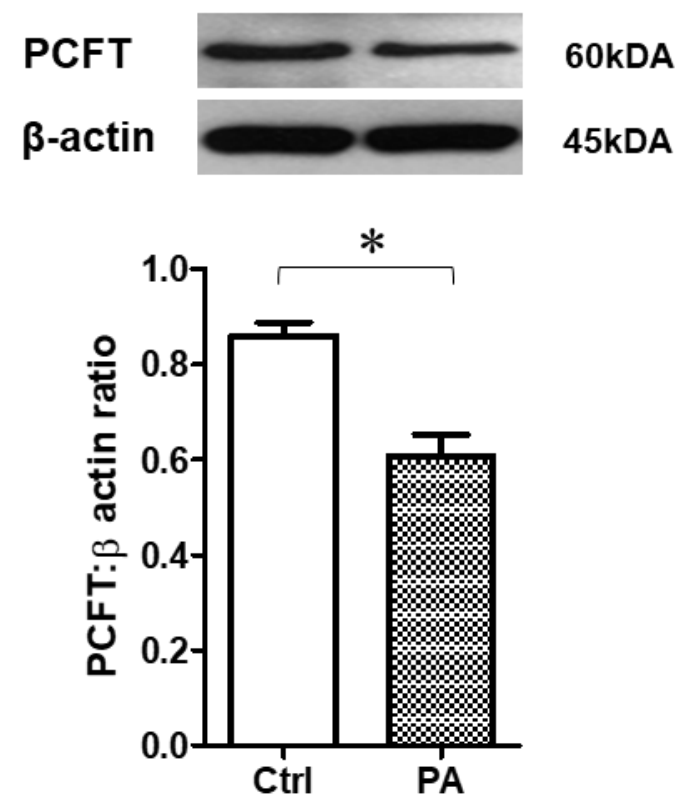

D
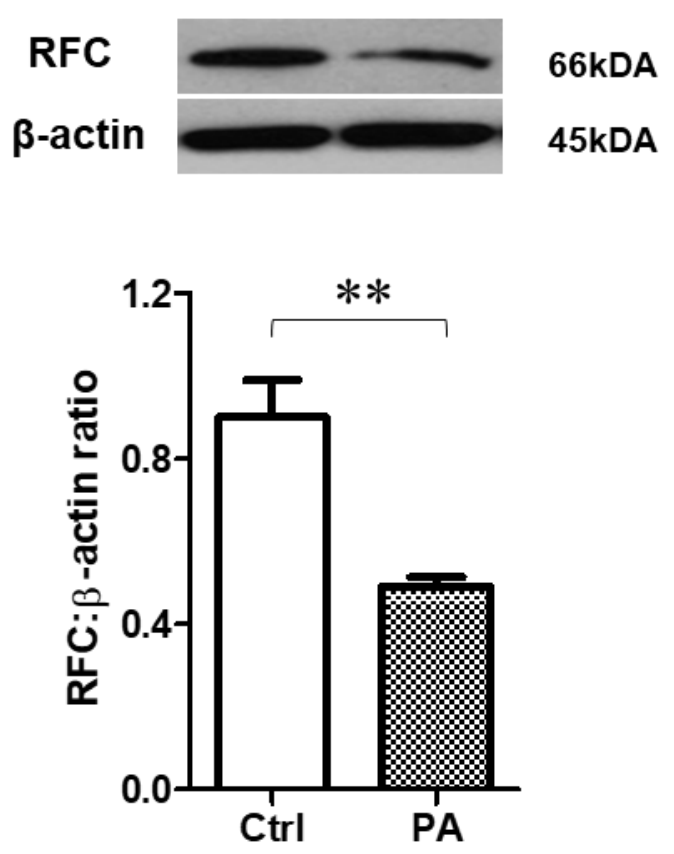


\section{Figure 3.8. Folate transporter expression and folate concentration in HepG2 cells}

Cells were incubated in the absence (control) or presence of palmitic acid (PA, $0.3 \mathrm{mM}$ ). (A) PCFT mRNA and (B) protein expression were measured. (C) RFC mRNA and (D) protein expression were measured. The mRNA levels were determined by real-time PCR analysis after $8 \mathrm{~h}$ incubation and the protein levels were determined by Western immunoblotting analysis after $12 \mathrm{~h}$ incubation. (E) Intracellular folate concentration was measured after $48 \mathrm{~h}$ incubation. Results are expressed as means \pm S.E. $(\mathrm{n}=4-6) .{ }^{*} p<0.05$ and ${ }^{* *} p<0.01$ when compared with the value obtained from the control cells. 
A
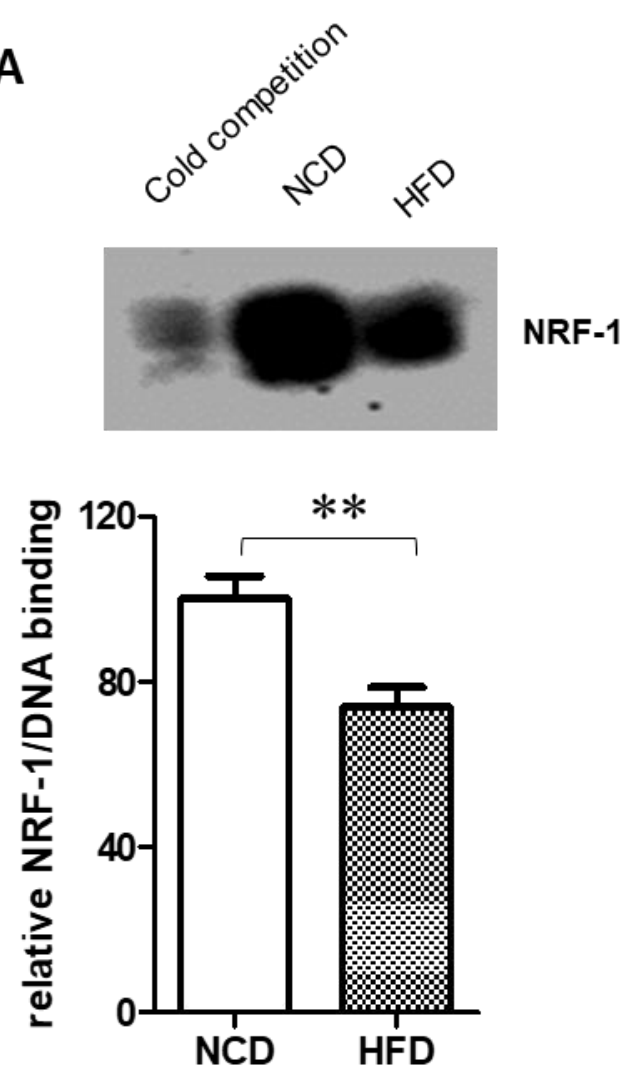

\begin{tabular}{|l|l|}
1 GCGCAGGCGCAGACAGCACAGACTGGTCCAGGCCTCCCGCAGCTGGTTCCGAGGTGCTAG \\
NRF-1 $\quad$ 5.UTR \\
61 CCCCTCCGTGTTGGGCATGGAGGGGCGCGTGAGCTCCGTGGGCTCGCCCCACAGTTTCC
\end{tabular} Mus musculus
C

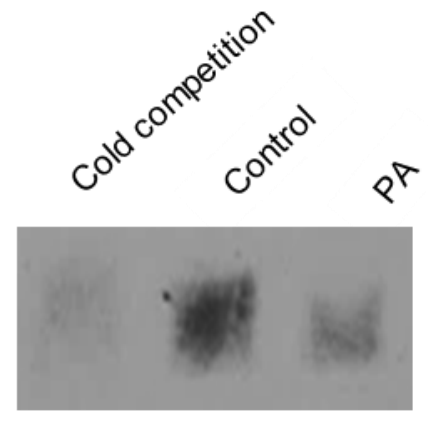

NRF-1

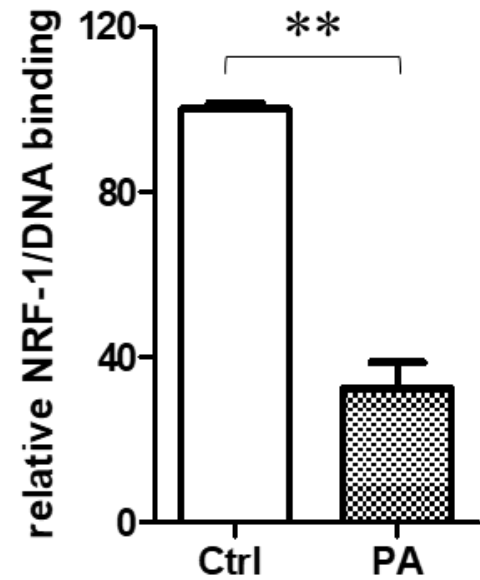

1921 CCGGGCGCAGGCGCAGACAGCGCAAGCCCCACGCCACGCGTCGCT GGT CCCAGGCAGCGA NRF-1

1981 GTCGCTCGCGCGCCCCGCCGCCCGCCTGGCGACAGCTCCGCC

D

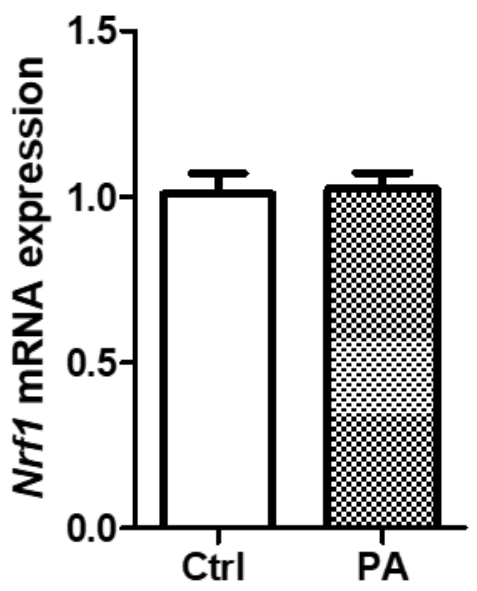




\section{Figure 3.9. Effect of high-fat diet on NRF-1/DNA binding activity in the liver}

Mice were fed a normal chow diet (NCD) or a high-fat diet (HFD) for 8 weeks. (A) The DNA binding activity of NRF-1 in the liver tissue was determined by EMSA. The binding activity in the NCD group was expressed as $100 \%$. The schematic diagram illustrates the promoter region of the PCFT gene in mouse (Mus musculus). The NRF-1 binding sequence is shown in bold letters and underlined. The 5'UTR site is indicated by an arrow. (B) The nuclear protein and DNA oligonucleotides were incubated with anti-NRF-1 antibodies $(2 \mu 1$ in lane $2,4 \mu 1$ in lane 3$)$ for supershift assay. The shift of the NRF-1/DNA complex is indicated by an arrow. (C) HepG2 cells were incubated with or without palmitic acid (PA, $0.3 \mathrm{mM})$ for $4 \mathrm{~h}$. The DNA binding activity of NRF-1 was determined by EMSA. The schematic diagram illustrates the promoter region of the PCFT gene in human (Homo sapiens). The NRF-1 binding sequence is shown in bold letters and underlined. The 5'UTR site is indicated by an arrow. (D) The NRF-1 mRNA in HepG2 cells was determined by real-time PCR analysis after $8 \mathrm{~h}$ incubation with or without palmitic acid. Results are expressed as means \pm S.E. $(\mathrm{n}=4-6) .{ }^{*} p<0.05$ and ${ }^{*} p<<0.01$ when compared with the value obtained from the control group. 
A

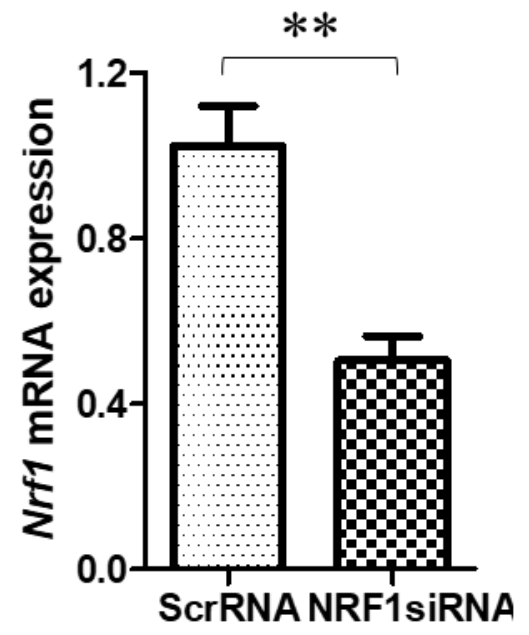

D
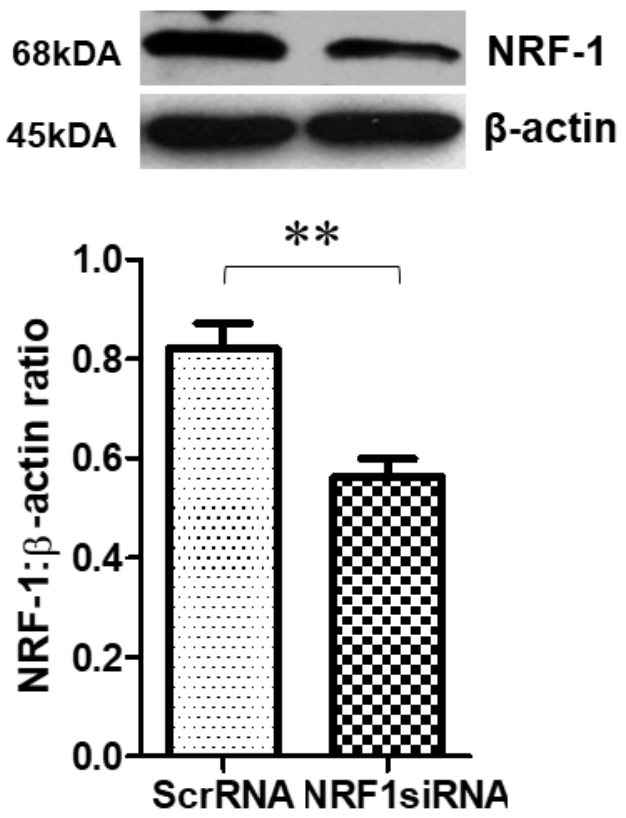

B

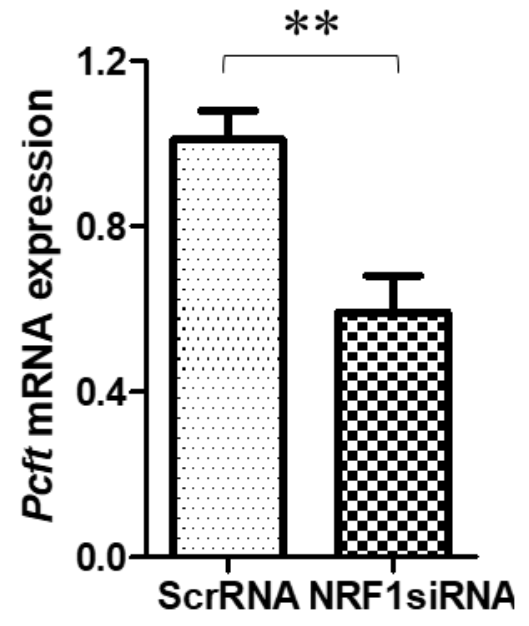

E
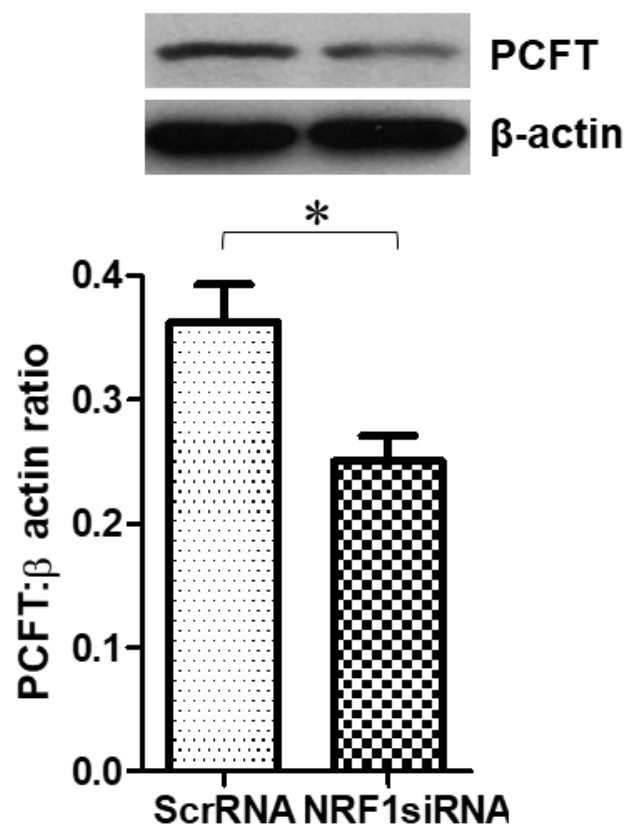

C

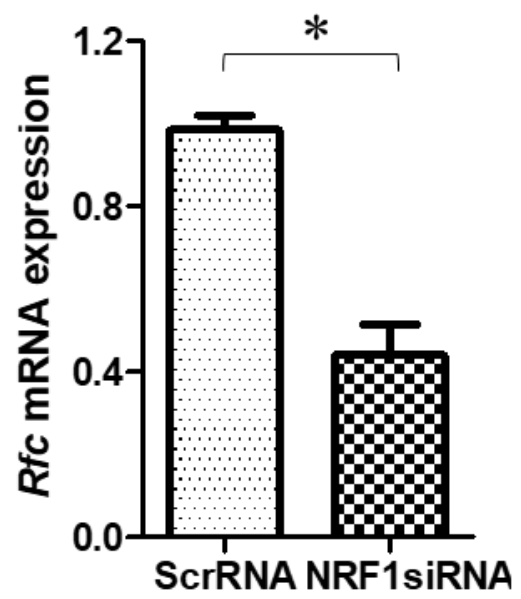

F
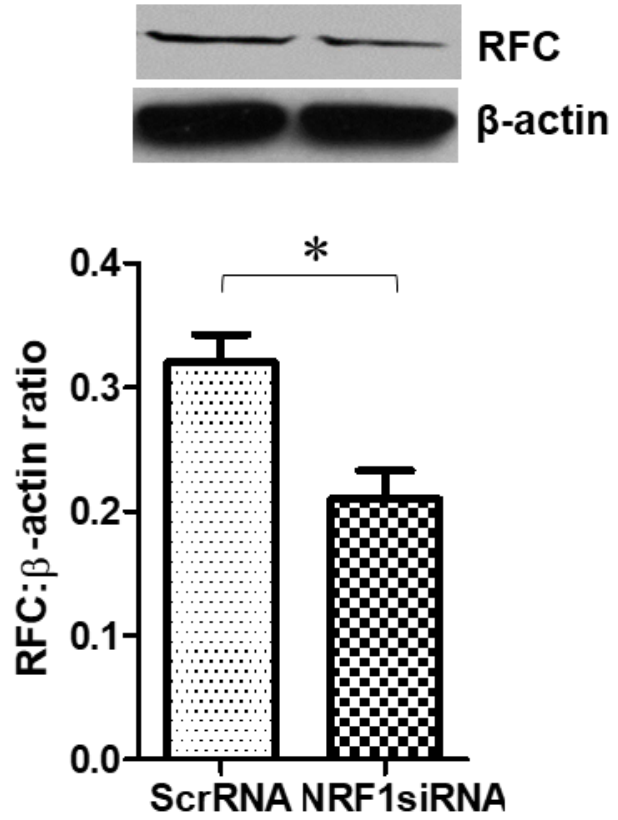

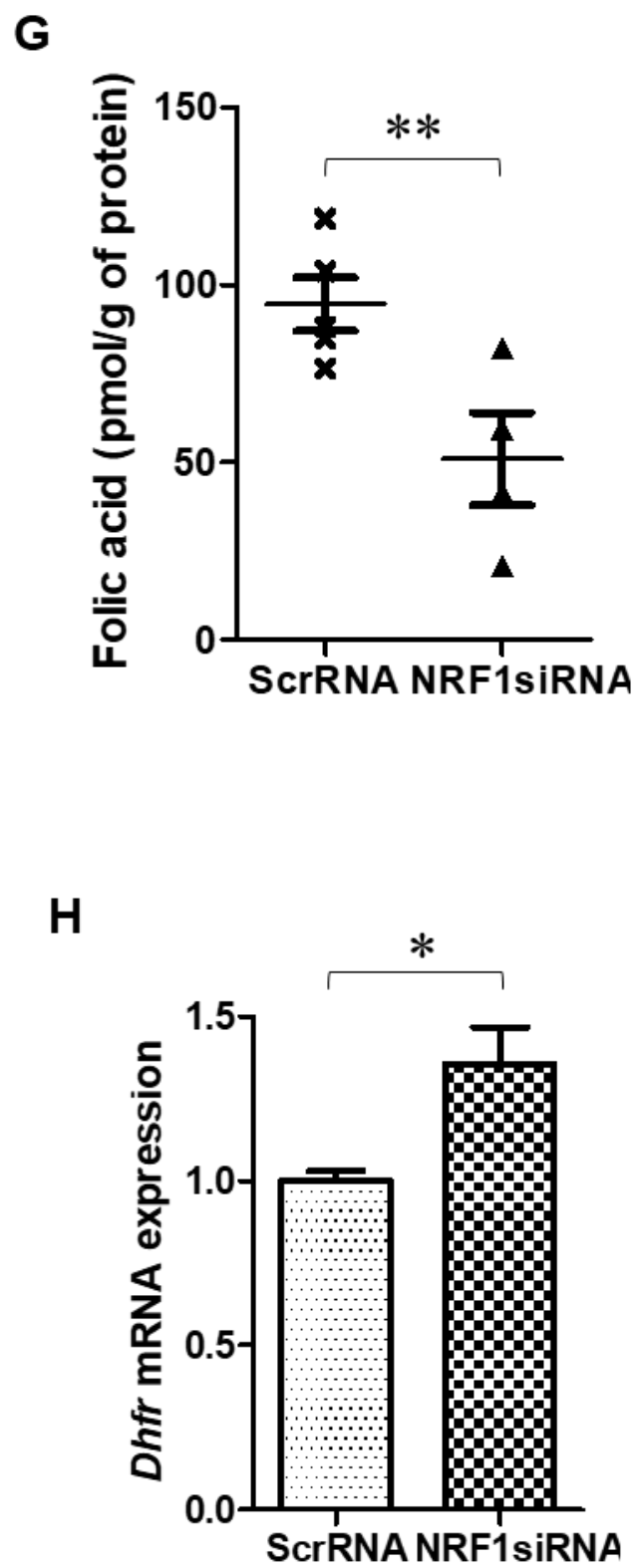

Figure 3.10. Effect of NRF-1 siRNA transfection on folate transporter expression and folate concentration in HepG2 cells

Cells were transfected with NRF-1 siRNA or scrambled siRNA (negative control). The mRNA levels of (A) NRF-1, (B) PCFT and (C) RFC were measured. The protein levels of (D) NRF-1, (E) PCFT and (F) RFC were measured. (G) Intracellular folate concentration was measured. (H) DHFR mRNA was measured. The mRNA and proteins were determined by real-time PCR and Western immunoblotting analysis, respectively. Results are expressed as means \pm S.E. $(n=4-6)$. $*_{p}<0.05$ and $* * p<0.01$ when compared with the value obtained from cells transfected with scrambled siRNA. 


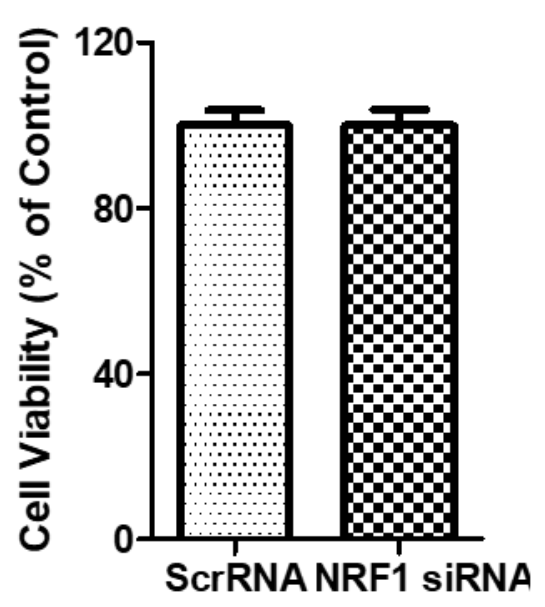

Figure 3.11. Effect of NRF-1 siRNA transfection on cell viability

Cells were transfected with NRF-1 siRNA or scrambled siRNA (negative control). Cell viability was assessed by incubating cells with 3-(4,5-dimethylthiazol-2-Yl)-2,5-diphenyltetrazolium bromide (MTT). The viability of cells transfected with scrambled siRNA was expressed as $100 \%$. Results are expressed as means \pm S.E. $(n=6)$. 

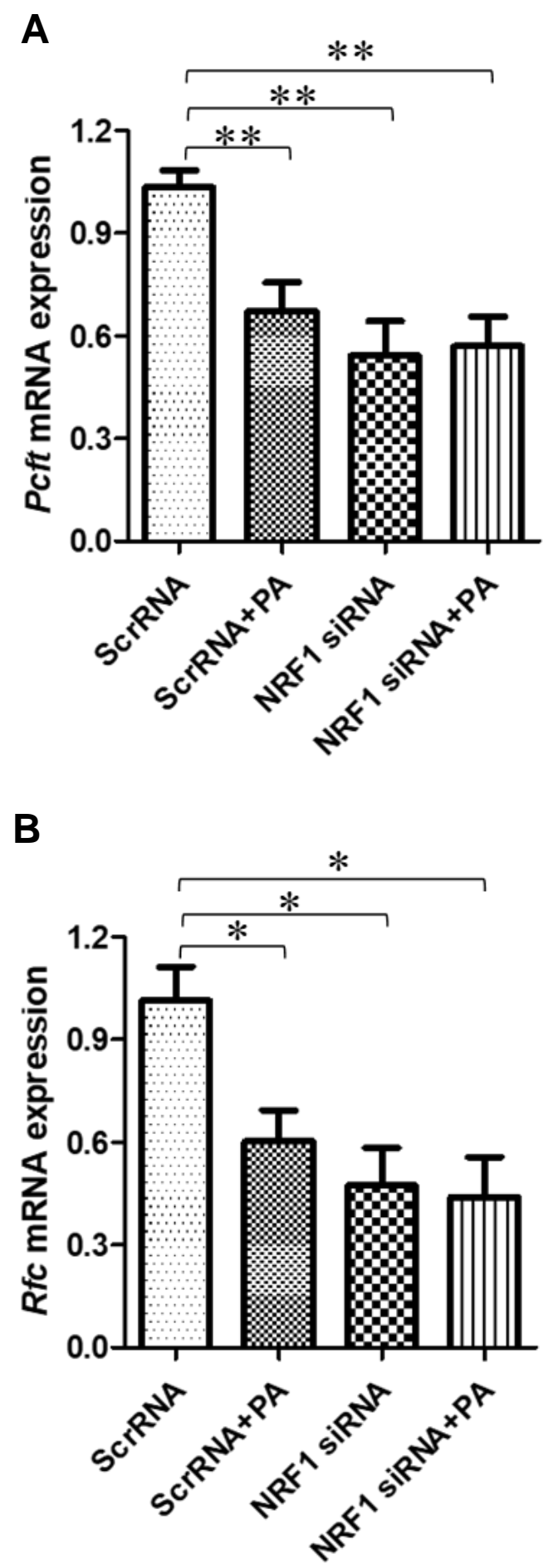

Figure 3.12. Effect of palmitic acid on folate transporter expression in NRF-1 transfected cells

Cells were transfected with NRF-1 siRNA or scrambled RNA (negative control). Following siRNA transfection, cells were either treated with or without palmitic acid (PA, 0.3mM). The mRNA levels of (A) PCFT and (B) RFC were measured. Results are expressed as means \pm S.E. $(\mathrm{n}=4) .{ }^{*} p<0.05$ and $* * p<0.01$ when compared with the value obtained from cells transfected with scrambled siRNA. 
Table 1. Primer sequences of folate transporters and other folate metabolism genes

\begin{tabular}{|l|l|l|}
\hline Gene & GenBank ${ }^{\text {TM }}$ Accession number & Primer sequences (5'-3') \\
\hline Mouse & & \\
\hline PCFT & NM_026740.2 & $\begin{array}{l}\text { F: CTCATGTTCACAGGGTACCG } \\
\text { R: CCGGGTAGATGGAGTTGAAG }\end{array}$ \\
\hline RFC & NM_031196.3 & $\begin{array}{l}\text { F: CCCATTGCCACTTTTCAGAT } \\
\text { R: CGCTTGTCAGAGACCACAAG }\end{array}$ \\
\hline Folr1 & NM_001252552.1 & $\begin{array}{l}\text { F: TACCCTAAGCAGGGAGTGGA } \\
\text { R: ACCTACTCGGAGCACAGGAA }\end{array}$ \\
\hline DHFR & NM_010049.3 & $\begin{array}{l}\text { F: AACTTGACGGCAATCCTAGC } \\
\text { R: GTACTTGAACTCGTTCCTGAGC }\end{array}$ \\
\hline ABCG2 & NM_001355477.1 & $\begin{array}{l}\text { F: CTGCTGATGTTGAAAGGCATAA } \\
\text { R: GAAGGCCGTTGTTGTTTCTC }\end{array}$ \\
\hline Mrp3 & NM_001363187.1 & F: CATACACAACGGCACCTTCA \\
R: TAGGCCACAGAGCCCTTTAC
\end{tabular}




\subsubsection{Discussion}

The present study, demonstrated that feeding mice a high-fat diet for 8 weeks impaired hepatic folate storage and reduced folate levels in the circulation. High-fat diet and saturated fatty acid suppressed the hepatic expression of two folate transporters, PCFT and RFC by interfere their transcription regulator, NRF-1, which may account for low folate status in obesity.

The liver is the primary organ for folate storage and is where folate is converted to its active form 5-methyltetrahydrofolate (5-MTHF) that is then delivered to peripheral tissues through the systemic or enterohepatic circulation (Steinberg et al. 1979, Wright et al. 2007, Sid et al. 2017). Folate transporters (PCFT, RFC) are abundantly expressed at the basolateral membrane of hepatocytes and mediate folate transport from the hepatic portal system to the liver (Zhao et al. 2009). Changes in hepatic folate transport not only affects folate storage in the liver but can also disrupt systemic folate homeostasis (Steinberg 1984). In the present study, mice fed a high-fat diet for 8 weeks developed fatty liver. Besides inducing hepatic lipid accumulation, high-fat diet feeding significantly reduced the expression of PCFT and RFC in the liver. Such a reduction of folate transporter expression was associated with low folate levels in the liver and serum. Furthermore, incubation of HepG2 cells with palmitic acid, a major saturated fatty acid in the high-fat diet, caused a decrease in folate transporter expression and intracellular folate levels. These results suggested that impaired expression of hepatic folate transporters might be one of the underlying mechanisms leading to low folate status in mice fed a high-fat diet.

The regulation of folate transporter expression in the liver is not well understood. The transcription factor NRF-1 was first identified to regulate gene expression of folate transporters in the intestine (Gonen et al. 2010). In the present study, we identified several lines of evidence that suggested the involvement of NRF-1 in the regulation of folate transporter expression in the 
liver. There was a significant reduction of NRF-1/DNA binding activity in the liver of mice fed a high-fat diet. This finding was consistent with decreased expression of PCFT and RFC in the liver. We then examined the role of NRF-1 in the transcriptional regulation of folate transporter expression in HepG2 cells. Inhibition of NRF-1 by using siRNA transfection significantly reduced folate transporter expression and folate concentrations in HepG2 cells. Although NRF-1 has been implicated as a key transcriptional factor that controls the expression of PCFT, its involvement in RFC expression has not been well-established. A recent study has shown that NRF-1 silencing not only reduces PCFT expression, but also significantly decreases RFC expression in HeLa cells (Gonen et al. 2010). However, the molecular mechanism by which NRF-1 is involved in RFC expression remains to be further investigated. The results from the present study suggest that downregulation of NRF-1 may contribute to decreased expression of folate transporters in the liver and reduced folate levels after chronic consumption of high-fat diets.

We previously reported that feeding mice with a high-fat diet-induced lipid accumulation and oxidative stress in the liver (Sarna et al. 2012, Wu et al. 2013). Several studies showed that dietary folate deficiency was associated with steatosis due to increased hepatic lipid biosynthesis and reduced lipid export via lipoproteins in mice (Christensen et al. 2010, Champier et al. 2012). A recent study reported that patients with NAFLD had low serum folic acid levels, which was associated with severity of steatosis and hepatocellular ballooning (Xia et al. 2018). In line with these clinical findings, the present study identified a low folate status that was associated with fatty liver caused by chronic consumption of a high-fat diet. Currently, effective treatment of patients with NAFLD remains a clinical challenge due to its multifaceted pathogenesis that is still incompletely understood (Loomba et al. 2013, Ahmed et al. 2015). We previously 
demonstrated that folic acid supplementation could counteract oxidative stress, and improve lipid and glucose metabolism in the liver of mice fed a high-fat diet (Sarna et al. 2012, Sid et al. 2015). These findings suggest that folate can exert hepatic protective effects in the context of diet-induced NAFLD. Obesity is the most common risk factor for NAFLD (Younossi et al. 2018) and obese patients often exhibit low folate levels in the circulation (Mojtabai 2004, Mahabir et al. 2008). It was previously reported that folate status was significantly improved in morbidly obese patients after gastric bypass surgery (Updegraff et al. 1981, Sid et al. 2017). Because treatment of obesity was able to restore folate levels in patients, this suggests that obesity may be the underlying cause for the imbalance of endogenous folate levels (Sid et al. 2017). Due to increased global prevalence of obesity, folate deficiency is expected to reoccur, which alarms both women in childbearing age and aging population. Further studies are warranted to investigate the relationship between obesity and folate status during pregnancy. Understanding the hepatic regulation of folate transporters and folate levels under pathophysiological conditions is imperative to ensure adequate folate status, which may be beneficial for patients with obesity and NAFLD.

In conclusion, the present study has demonstrated that chronic consumption of high-fat diets reduces the expression of hepatic folate transporters (PCFT, RFC), which, in turn, may account for low folate levels in the liver and circulation. Downregulation of transcription factor NRF-1 may be responsible for reduced expression of hepatic PCFT and RFC. These novel findings suggest that regulation of folate transporter expression in the liver is important in maintaining adequate folate status in the body. As a key nutrient, folate is essential for many biological functions in humans and animals. Although the general populations in Western countries can achieve sufficient dietary folate intakes, findings from our study indicate that prolonged 
consumption of diets that are high in fats may exert a negative influence on folate homeostasis.

A better understanding of the mechanism by which high-fat diets disrupts hepatic folate transporter expression may have a significant implication for management folate deficiency. 


\section{STUDY 2 - MANUSCRIPT 2}

3.2. Folic acid supplementation during high-fat diet feeding restores AMPactivated protein kinase (AMPK) activation via an AMP-LKB1 dependent mechanism

V. Sid, N. Wu, L.K. Sarna, Y.L. Siow, J.D. House, K. O. Am J Physiol Regul Integr Comp Physiol. 309(10): R1215-1225 (2015) 


\subsubsection{Abstract}

AMP-activated protein kinase (AMPK) is an endogenous energy sensor that regulates lipid and carbohydrate metabolism. Non-alcoholic fatty liver disease (NAFLD) is regarded as a hepatic manifestation of metabolic syndrome with impaired lipid and glucose metabolism, and increased oxidative stress. Our recent study showed that folic acid supplementation attenuated hepatic oxidative stress and lipid accumulation in high-fat diet fed mice. The aim of the present study was to investigate the effect of folic acid on hepatic AMPK during high-fat diet feeding and the mechanisms involved. Male C57BL/6J mice were fed a control diet (10\% kcals fat), a high-fat diet $(60 \%$ kcals fat) or a high-fat diet supplemented with folic acid (26mg/kg diet) for 5 weeks. Mice fed a high-fat diet exhibited hyperglycemia, hepatic cholesterol accumulation and reduced hepatic AMPK phosphorylation. Folic acid supplementation restored AMPK phosphorylation (activation), and reduced blood glucose and hepatic cholesterol levels. Activation of AMPK by folic acid was mediated through an elevation of its allosteric activator AMP and activation of its upstream kinase, namely, liver kinase B1 (LKB1) in the liver. Consistent with in vivo findings, 5-methyltetrahydrofolate (bioactive form of folate) restored phosphorylation (activation) of both AMPK and LKB1 in palmitic acid-treated HepG2 cells. Activation of AMPK by folic acid might be responsible for AMPK-dependent phosphorylation of HMG-CoA reductase, leading to reduced hepatic cholesterol synthesis during high-fat diet feeding. These results suggest that folic acid supplementation may improve cholesterol and glucose metabolism by restoration of AMPK activation in the liver. 


\subsubsection{Introduction}

AMPK is a master regulator of whole body energy balance and metabolic homeostasis. It modulates anabolic and catabolic pathways involved in carbohydrate, lipid and protein metabolism, through phosphorylation of downstream enzymatic and transcriptional mediators (Viollet et al. 2009, Canto et al. 2010, Hardie et al. 2012). AMPK is a heterotrimeric complex that is composed of 3 subunits, namely a catalytic $\alpha$ subunit and regulatory subunits ( $\beta$ and $\gamma)$. AMPK is activated by allosteric AMP interaction with its $\gamma$ subunit (at adenine binding sites) and by phosphorylation (at Thr-172) of the $\alpha$ subunit via upstream kinases (Hawley et al. 1996, Stein et al. 2000, Gowans et al. 2013). Although several kinases have been identified, liver kinase B1 (LKB1) is thought to be the predominant upstream kinase that is responsible for phosphorylation of AMPK (activation) in the liver (Woods et al. 2003). Phosphorylation of LKB1 (Ser-428 in human or Ser-431 in mouse) is important for its activation (Hou et al. 2008, Yoneda et al. 2010, Gan et al. 2014). Upstream enzymes including sirtuin 1 (SIRT1), protein kinase A (PKA) and protein kinase C-zeta (PKC- $\zeta$ ) have been implicated in LKB1 activation, which, in turn leads to activation of AMPK (Collins et al. 2000, Hou et al. 2008, Xie et al. 2008). Since AMPK regulates metabolic pathways involved in glucose and lipid metabolism, it has been proposed as a potential therapeutic target in metabolic diseases such as obesity, type 2 diabetes mellitus and non-alcoholic fatty liver disease (NAFLD) (Viollet et al. 2006, Steinberg et al. 2009).

NAFLD is regarded as a hepatic manifestation of metabolic syndrome and is characterized by impaired lipid and glucose metabolism, and increased oxidative stress in the liver. The spectrum of NAFLD ranges from steatosis (lipid accumulation in the liver), to non-alcoholic steatohepatitis (NASH) and hepatic cirrhosis in its advanced stages (Farrell et al. 2006, Tiniakos et al. 2010). Chronic consumption of high-fat diets is associated with obesity and NAFLD 
(Golay et al. 1997, Angulo 2007, Hariri et al. 2010). The high-fat diet rodent model develops hepatic histopathological features in context of the metabolic syndrome such as hyperglycemia and abnormal lipid metabolism, and therefore is commonly used to investigate the pathogenesis of NAFLD (Marchesini et al. 2003, Hebbard et al. 2011, Sarna et al. 2012, Takahashi et al. 2012). It is evident that hepatic regulation of glucose and cholesterol metabolism is also perturbed in high-fat diet fed rodents (Pu et al. 2012, Wu et al. 2013, Zhang et al. 2013), which is associated with a decrease in AMPK activation in the liver (Pu et al. 2012, Zhang et al. 2013). However, the regulation of hepatic AMPK during high-fat diet feeding is poorly understood. Understanding AMPK regulation in the liver during high-fat diet consumption might be important for improving glucose and cholesterol metabolism, which are often dysregulated in NAFLD (Marchesini et al. 2003, Min et al. 2012, Wu et al. 2013).

Folate is a naturally occurring water-soluble $\mathrm{B}$ vitamin. It participates in intracellular methylation and one-carbon metabolism reactions, and also contributes to nucleotide and amino acid biosynthesis in the body. Folic acid is the synthetic form of folate with a greater stability, and is commonly fortified in foods and used for supplementation (Lucock 2000, Iyer et al. 2009). Folic acid fortification of the diet has been implemented to reduce the incidence of neural tube defects in newborns (Kim 2007). The liver is the major organ responsible for folate storage and metabolism (Lucock 2000). Although folic acid supplied by a typical Western diet is sufficient to meet the requirements of generally healthy individuals, studies have revealed that serum folate levels are inversely correlated with obesity and are associated with the manifestation of liver disorders (Leevy et al. 1970, Leevy et al. 1970, Mahabir et al. 2008). Diets deficient in lipotropes such as methionine, choline and/or folic acid have been shown to induce hepatic steatosis (Christensen et al. 2010, Hebbard et al. 2011). Supplementation with methyl donors (choline, 
methionine, vitamin B12 and folic acid) can attenuate hepatic lipid accumulation in high-fat diet fed mice and may reduce the progression of NAFLD (Dahlhoff et al. 2014). In our previous study, we have observed that folic acid supplementation is hepatoprotective through reducing oxidative stress and lipid accumulation in the liver of high-fat diet fed mice (Sarna et al. 2012) as well as in hyperhomocysteinemic rats (Woo et al. 2006). The ability for folic acid to minimize lipid accumulation in the liver suggests that it may regulate hepatic lipid metabolism, but the underlying mechanisms remain to be defined. Since AMPK plays a crucial role in metabolic regulation, we hypothesize that folic acid supplementation may promote the activation of hepatic AMPK in high-fat diet fed mice. In the present study, we aimed to investigate the mechanisms by which folic acid regulated AMPK in the liver during high-fat diet feeding. 


\subsubsection{Materials and Methods}

i) Animal model

Male C57Bl/6J mice aged 6 weeks (The Jackson Laboratory, Bar Harbour, Maine, USA) were fed either a control diet, high-fat diet or high-fat diet supplemented with folic acid for 5 weeks (Hwang et al. 2013, Wu et al. 2013). The formulation of the purified diets were as follows: control diet (D12450B) consisted of 10\% kcals fat, $20 \%$ kcals protein and $70 \%$ kcals carbohydrate with $2 \mathrm{mg}$ of folic acid per $\mathrm{kg}$ of diet $(3.85 \mathrm{kcal} / \mathrm{g})$; high-fat diet (D12492) contained $60 \%$ kcals fat, $20 \%$ kcals protein and $20 \%$ kcals of carbohydrate with $2.6 \mathrm{mg}$ of folic acid per $\mathrm{kg}$ of diet $(5.24 \mathrm{kcal} / \mathrm{g})$; and high-fat diet supplemented with folic acid consisted of $26 \mathrm{mg}$ of folic acid per $\mathrm{kg}$ of diet (Table 2). The sources of fat in both the control and high-fat diet are soybean oil and lard (Research Diets Inc). The fat content in the high-fat diet is mainly derived from lard, while the main source of fat in the control diet is soybean oil (Table 2). In a pilot study, we conducted experiments in high-fat diet fed mice that were supplemented with various doses of folic acid $(5.6,13,26,65$ and 130mg per $\mathrm{kg}$ of diet). We observed that increasing the folic acid contents to 26,65 or $130 \mathrm{mg}$ per $\mathrm{kg}$ of diet had glucose lowering effects in high-fat diet fed mice. In the present study, we used $26 \mathrm{mg}$ per $\mathrm{kg}$ folic acid for supplementation, which was the lowest dose that displayed glucose-lowering effects in high-fat diet fed mice. All the diets were prepared by the Research Diets Inc. (Hwang et al. 2013, Wu et al. 2013). The mice were maintained on a 12-hour light/12-hour dark cycle and had free accessibility to water and food. Body weights were recorded prior to feeding and at the end of the experimental feeding period. At the end of the 5 week period, mice were killed and the blood was collected for serum preparation. The liver tissue was quickly removed and placed in liquid nitrogen, then frozen at $-80^{\circ} \mathrm{C}$ until further analysis. Blood glucose was measured at the 
beginning and ending of the 5 week feeding period. Mice were fasted for 5 hours, and blood was sampled through the tail vein for glucose measurement. All procedures were performed in accordance with the Guide to the Care and Use of Experimental Animals published by the Canadian Council on Animal Care and approved by the University of Manitoba Protocol Management and Review Committee.

\section{ii) Cell culture}

HepG2 cells (human hepatoblastoma cell line HB-8065; American Type Culture Collection, MA) were cultured in high glucose Dulbecco's modified Eagle's medium (Hyclone) supplemented with $10 \%$ FBS in a humidified incubator at $37^{\circ} \mathrm{C}$ with $5 \% \mathrm{CO}_{2}$. $\mathrm{HepG} 2$ cells resemble many characteristics of hepatocytes and are widely used as a valuable model in metabolic and pharmacological studies (Javitt 1990, Wilkening et al. 2003, Woo et al. 2006, Wu et al. 2013). HepG2 cells have also been used in studies of folate uptake and folate-dependent metabolism (Qiu et al. 2006, Wang et al. 2011). Treatments included palmitic acid (Sigma Aldrich), 5-methyltetrahydrofolate (5-MTHF; Sigma Aldrich) and folic acid (Sigma Aldrich). Palmitic acid was dissolved in $10 \%$ BSA and $5 \%$ ethanol with light shaking overnight at $37^{\circ} \mathrm{C}$. Palmitic acid is the most abundant saturated fatty acid in the high-fat diet. It is also the major circulating saturated fatty acid in NAFLD patients (Puri et al. 2007) and in mice fed a high-fat diet (Buettner et al. 2007). Palmitic acid (0.3mM) has been shown to induce lipid accumulation in HepG2 cells, which was used as an in vitro model to study NAFLD pathogenesis (Joshi-Barve et al. 2007, Wu et al. 2013). 5-MTHF is the predominant form of folate detected in the circulation in humans and rodents. It is also the active form of folate that is taken up by the liver for storage and distribution to peripheral tissues (Zhao et al. 2009, Pietrzik et al. 2010). It has 
been reported in humans that folic acid can also enter the hepatic portal circulation following intestinal absorption and is reduced to 5-MTHF in the liver (Wright et al. 2007). In a pilot study, we tested palmitic acid, 5-MTHF and folic acid at various doses in HepG2 cells. We observed that palmitic acid $(0.3 \mathrm{mM})$ reduced AMPK phosphorylation, while 5-MTHF $(1 \mu \mathrm{g} / \mathrm{ml})$ and folic acid $(1 \mu \mathrm{g} / \mathrm{ml})$ could restore AMPK activation. In the subsequent experiments, palmitic acid $(0.3 \mathrm{mM}), 5-\mathrm{MTHF}(1 \mu \mathrm{g} / \mathrm{ml})$ and folic acid $(1 \mu \mathrm{g} / \mathrm{ml})$ were used. In some experiments, nicotinamide (Sigma Aldrich), N-[2-(p-bromocinnamylamino)ethyl]-5-isoquinolinesulfonamide dihydrochloride (H89; Sigma Aldrich), PKC- $\zeta$ pseudosubstrate (Sigma Aldrich), AMP sodium salt (Sigma Aldrich) and compound C (Calbiochem) were simultaneously incubated in cells treated with palmitic acid and/or 5-MTHF. The concentrations of the inhibitors or activators were tested to identify the lowest dose that could induce detectable changes in AMPK and LKB1 phosphorylation. Nicotinamide $(10 \mathrm{mM})$ was used to inhibit SIRT1, an upstream deacetylase that

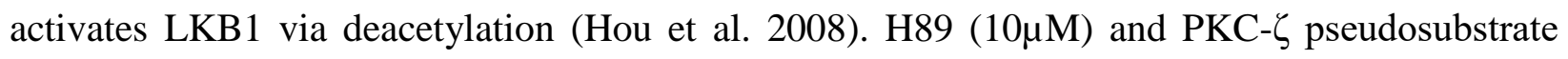
$(1 \mu \mathrm{M})$ were used to suppress PKA and PKC- $\zeta$ activation respectively (Xie et al. 2008). Compound $\mathrm{C}(10 \mu \mathrm{M})$ was used to inhibit activation of AMPK (Wu et al. 2013) and AMP $(100 \mu \mathrm{M})$ was used to stimulate AMPK activation (Gowans et al. 2013).

iii) Western immunoblotting analysis

Protein levels of phosphorylated and total AMPK, phosphorylated and total LKB1, and phosphorylated and total HMG-CoA reductase were determined by Western immunoblotting analysis. In brief, liver proteins $(70 \mu \mathrm{g})$ were separated by electrophoresis in an $8 \%$ or $10 \%$ SDS polyacrylamide gel. Subsequently, proteins were transferred from the gel to a nitrocellulose membrane, which was then incubated with primary antibody overnight at $4^{\circ} \mathrm{C}$. Primary 
antibodies include rabbit anti-AMPK $\alpha$ polyclonal antibody (New England Biolabs), rabbit antiphospho-AMPKa (Thr-172) polyclonal antibody (New England Biolabs), rabbit anti-LKB1 monoclonal antibody (New England Biolabs), rabbit anti-phospho-LKB1 (Ser-428/431) monoclonal antibody (New England Biolabs) and rabbit anti-phospho-HMG-CoA reductase (Ser-872) polyclonal antibody (Millipore). HRP-conjugated anti-mouse or anti-rabbit IgG antibodies (New England Biolabs) were used as secondary antibodies. Protein bands were detected in chemiluminescent HRP detection reagent (Luminata Crescendo; Millipore) for visualization. To ensure equal protein loading, the same membranes were re-probed with mouse anti- $\beta$-actin monoclonal antibody (New England Biolabs).

iv) Determination of AMP levels

The AMP in the liver tissue was measured by using the AMP-Glo ${ }^{\mathrm{TM}}$ Assay kit (Promega, Madison, WI). The values were expressed in relative luminescence units (RLU), which was proportional to the AMP concentration. Data was plotted as percentage of control based on the changes in RLU ( $\triangle R L U$ ) calculated from the standard curve.

v) Measurement of cholesterol and HMG-CoA reductase activity

Lipids in the liver tissue were extracted according to the Folch method (Folch et al. 1957). The levels of cholesterol in the liver and hepatocytes were determined by using enzymatic kits (Wako Chemicals) (Wu et al. 2011, Wu et al. 2013). HMG-CoA reductase activity in the liver microsomes was measured by using $\left[3-{ }^{14} \mathrm{C}\right]$ HMG-CoA (PerkinElmer) as a substrate (Wu et al. 2011, Wu et al. 2013). 
vi) Statistical analysis

Results were analyzed using one-way ANOVA followed by Newman-Keuls post hoc test. $P$ values less than 0.05 were considered statistically significant. 


\subsubsection{Results}

i) Body weight and biochemical parameters of mice

Mice fed a high-fat diet for 5 weeks exhibited a significant increase in body weight, similar to the results observed in our previous studies (Hwang et al. 2013, Wu et al. 2013). Folic acid supplementation had no effect on body weight gain in these animals (Fig. 3.13A). High-fat diet consumption for 5 weeks was accompanied by elevated fasting blood glucose (Fig. 3.13B) and insulin levels (Fig. 3.13C). Folic acid supplementation attenuated an increase in fasting blood glucose levels (Fig. 3.13B) in mice fed a high-fat diet but did not significantly reduce fasting blood insulin levels (Fig. 3.13C). In addition, mice fed a high-fat diet exhibited higher levels of total cholesterol (Fig. 3.13D), free cholesterol (Fig. 3.13E) and triglyceride (Fig. 3.13F) in the liver. Folic acid supplementation during high-fat diet feeding reduced total and free cholesterol levels (Fig. 3.13D, 3.13E), but did not significantly change the triglyceride content in the liver (Fig. 3.13F).

ii) Regulation of hepatic AMPK and LKB1 in mice

In general, phosphorylation of AMPK $\alpha$ at Thr-172 leads to kinase activation while dephosphorylation inactivates it. Western immunoblotting of phosphorylated AMPK is a recognized surrogate for AMPK activity (Stein et al. 2000). Relative to the control group, the protein levels of phosphorylated AMPK were markedly reduced (inactivation) in the liver of high-fat diet fed mice, while total AMPK protein levels remained constant in all the groups (Fig. 3.14A). Under the same dietary condition, folic acid supplementation increased AMPK phosphorylation (activation) in the liver (Fig. 3.14A). Consistent with AMPK inactivation, highfat diet feeding caused a significant reduction in LKB1 phosphorylation (Fig. 3.14B). By 
contrast, folic acid supplementation markedly enhanced phosphorylation of LKB1 (activation), while total LKB1 levels were unchanged among the groups (Fig. 3.14B). These results suggested that folic acid may stimulate hepatic AMPK activation during high-fat diet consumption through inducing LKB1 activation.

iii) Effect of fatty acid and folic acid on AMPK and LKB1 activation in hepatocytes

To investigate the mechanisms by which folic acid supplementation regulated hepatic LKB1 and AMPK activation, experiments were performed in HepG2 cells that were incubated with palmitic acid. The protein level of phosphorylated AMPK was decreased in cells incubated with palmitic acid (Fig. 3.15A), indicating inactivation of the kinase. Incubation of cells with 5-MTHF effectively restored AMPK phosphorylation status in palmitic acid-treated cells, while total AMPK protein levels remained constant in all the groups (Fig. 3.15A). In another set of experiments, incubation of cells with folic acid also restored AMPK phosphorylation status in palmitic acid-treated cells (Fig. 3.15B). As a control, experiments were conducted in HepG2 cells to examine the effect of 5-MTHF or folic acid without the influence of palmitic acid. Folic acid or 5-MTHF treatment did not alter AMPK phosphorylation status in control cells (Fig. 3.15C). As both 5-MTHF and folic acid could restore AMPK phosphorylation in palmitic acidtreated cells, 5-MTHF was used in the subsequent experiments. The level of phosphorylated LKB1 protein was significantly decreased in cells incubated with palmitic acid (Fig. 3.15D). Incubation of cells with 5-MTHF restored LKB1 phosphorylation status in palmitic acid-treated cells, while total LKB1 levels remained constant among the groups (Fig. 3.15D). Several inhibitors were employed to identify potential upstream targets that might be involved in LKB1 activation by 5-MTHF. Nicotinamide is an inhibitor of sirtuin 1 (SIRT 1) (Avalos et al. 2005, 
Hou et al. 2008) which is an upstream deacetylase that promotes LKB1 activation (Hou et al. 2008, Lan et al. 2008). Though nicotinamide treatment slightly lowered 5-MTHF-induced phosphorylation of LKB1 in palmitic acid-treated cells, the effect was not significant (Fig. 3.16). PKA and PKC- $\zeta$ can also activate LKB1 via phosphorylation at its Ser-428 (Collins et al. 2000, Xie et al. 2008). To determine whether 5-MTHF-induced LKB1 phosphorylation was regulated by these kinases, hepatocytes were incubated with $\mathrm{H} 89$ (inhibitor of PKA) or PKC- $\zeta$

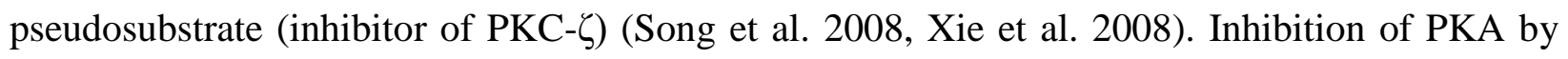
H89 abolished 5-MTHF-induced LKB1 phosphorylation in hepatocytes, while incubation with the PKC- $\zeta$ inhibitor did not cause a significant change in LKB1 phosphorylation status (Fig. 3.16). Taken together, these results suggested that PKA might be involved in 5-MTHF-induced LKB1 activation in hepatocytes.

iv) Role of AMP on AMPK activation in hepatocytes and liver tissue

Aside from LKB1, AMPK is allosterically activated by AMP. Compound C is a selective inhibitor of AMPK that competes with adenine nucleotide binding on the kinase (Zhou et al. 2001). Incubation of hepatocytes with compound C strongly suppressed 5-MTHF-induced AMPK phosphorylation (Fig. 3.17A). However, AMP effectively restored the phosphorylation of AMPK in palmitic acid-treated cells (Fig. 3.17A). In addition, AMP levels were measured in the liver tissue. While high-fat diet feeding caused a significant reduction in hepatic AMP levels, folic acid supplementation markedly increased AMP levels in the liver of mice fed a high-fat diet (Fig. 3.17B). These results indicated that under conditions of fatty acid overload or high-fat diet consumption, folic acid supplementation might also restore AMPK activation in the liver through the elevation of hepatic AMP levels. 
v) Effect of folic acid supplementation on hepatic cholesterol production

HMG-CoA reductase regulates the rate-limiting step in cholesterol biosynthesis (Wu et al. 2011, $\mathrm{Wu}$ et al. 2013). AMPK is identified as the major upstream kinase responsible for phosphorylation of HMG-CoA reductase (inactivation) (Omkumar et al. 1994, Jurevics et al. 2000). High-fat diet feeding decreased the levels of phosphorylated HMG-CoA reductase in the liver (Fig. 3.18A), which was accompanied with a significant increase in HMG-CoA reductase activity (Fig. 3.18B). In contrast, folic acid supplementation increased HMG-CoA reductase phosphorylation (Fig. 3.18A) and reduced the activity of the reductase in high-fat diet fed mice (Fig. 3.18B). Furthermore, incubation of HepG2 cells with palmitic acid resulted in a significant increase in cellular total cholesterol levels, while 5-MTHF treatment effectively reduced total cholesterol levels in palmitic acid-treated cells (Fig. 3.19). These results suggested that inhibition of HMG-CoA reductase activity by folic acid might be mediated through AMPK-dependent phosphorylation of the reductase. 
Table 2. Composition of purified diets: energy, macronutrient and folic acid content

\begin{tabular}{|c|c|c|c|}
\hline & $\begin{array}{c}\text { Control } \\
\text { (D12450B) }\end{array}$ & $\begin{array}{c}\text { High Fat Diet } \\
\text { (D12492) }\end{array}$ & $\begin{array}{l}\text { High Fat Diet }+ \\
\text { 10x Folic acid }\end{array}$ \\
\hline \multicolumn{4}{|l|}{ Energy content (\% kcal) } \\
\hline Protein & 20 & 20 & 20 \\
\hline Carbohydrate & 70 & 20 & 20 \\
\hline Fat & 10 & 60 & 60 \\
\hline \multicolumn{4}{|l|}{ Fat content $(\mathrm{g} / \mathrm{kg})$} \\
\hline Soybean oil & 24 & 32 & 32 \\
\hline Lard & 19 & 317 & 317 \\
\hline Folic acid (mg/kg) & 2 & 2.6 & 26 \\
\hline Dietary energy (kcal/g) & 3.85 & 5.24 & 5.24 \\
\hline
\end{tabular}


A

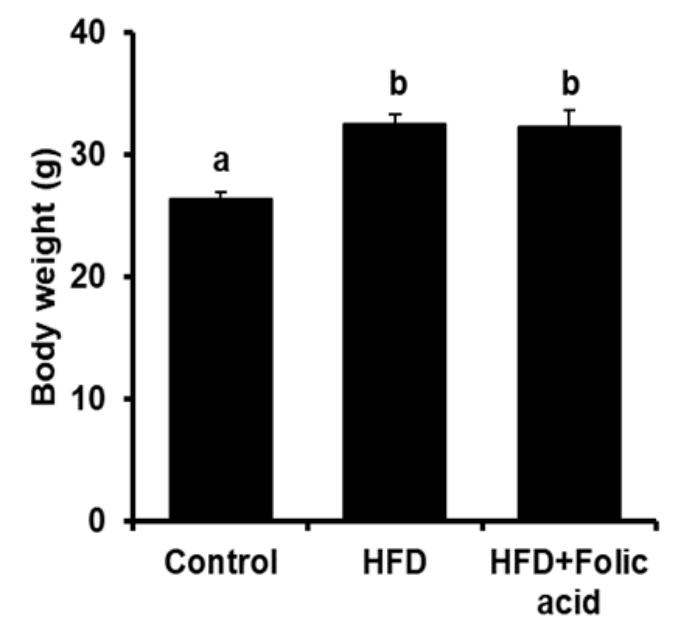

C

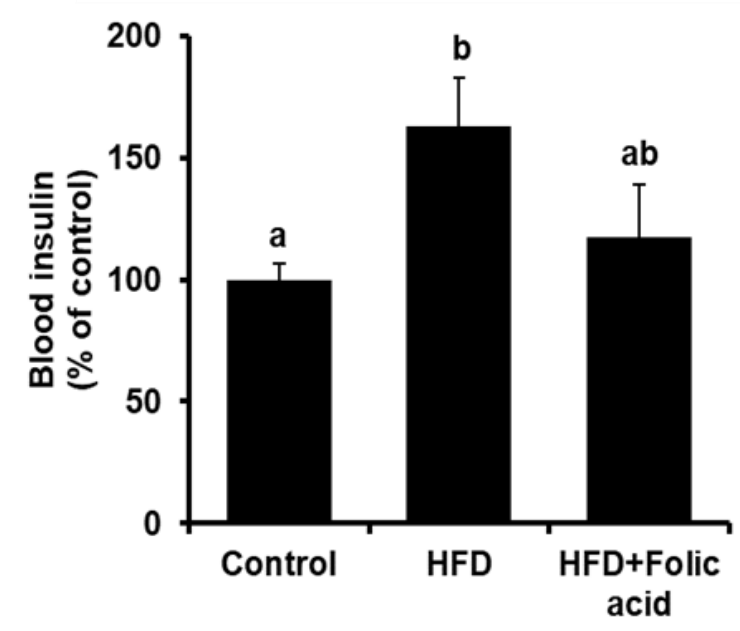

B

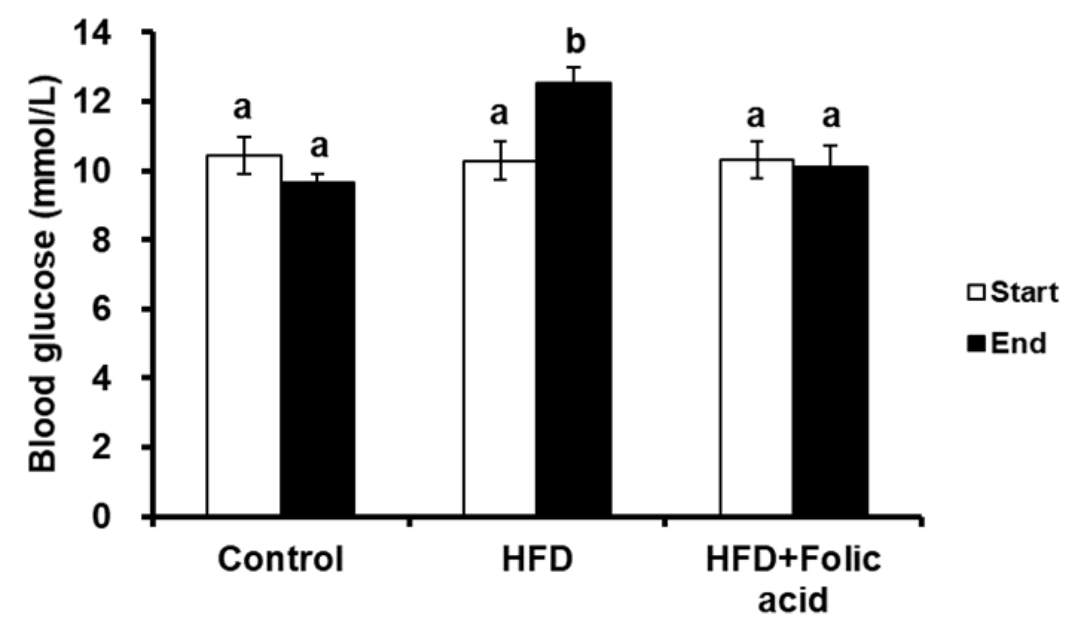

D

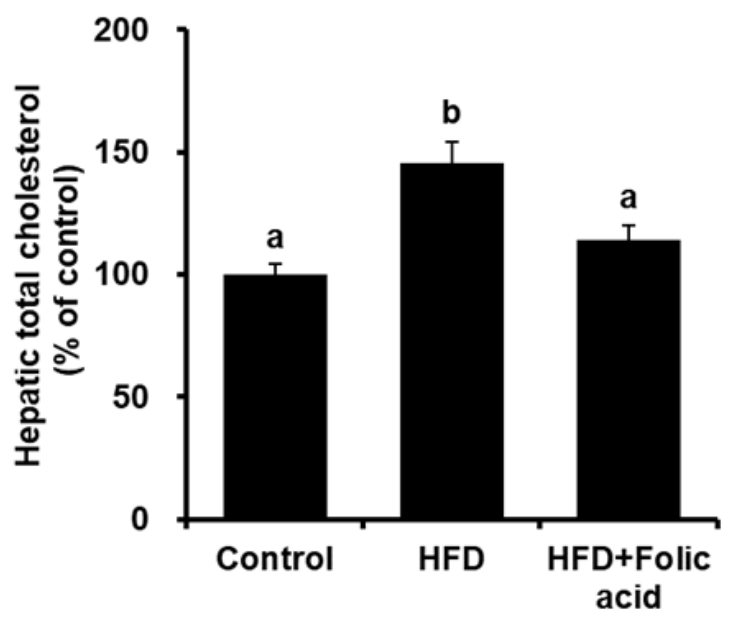


E

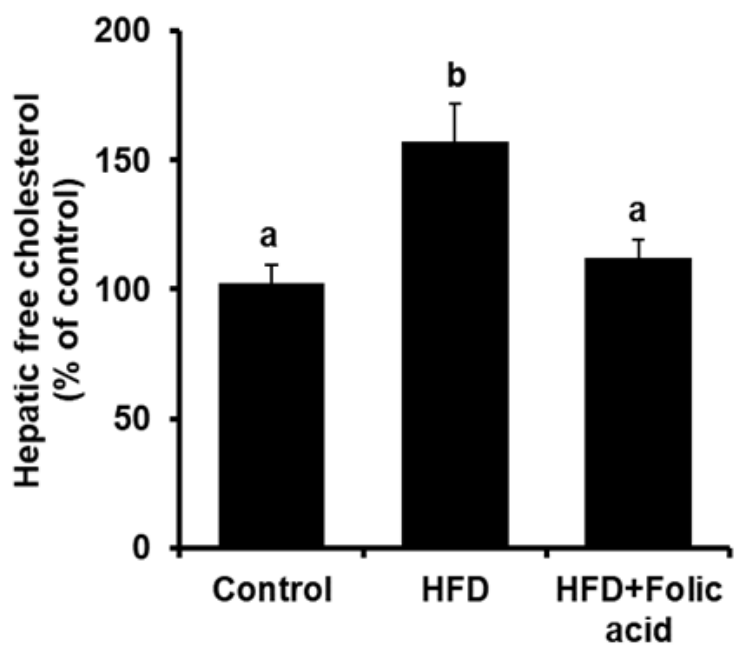

$\mathbf{F}$

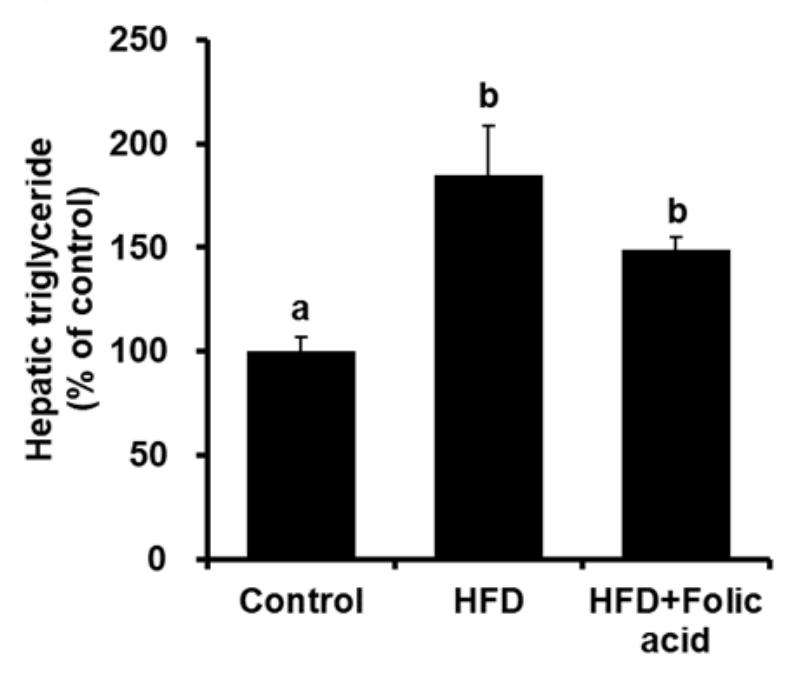

\section{Figure 3.13. Body weight, blood glucose and liver lipids}

Mice were fed a control diet, a high-fat diet (HFD) or a high-fat diet supplemented with folic acid (HFD+Folic acid) for 5 weeks. (A) Body weight was measured at the end of the 5 week feeding period. (B) Fasting blood glucose was measured before and after the 5 week feeding period. Open bars indicate the start of the feeding period while black bars indicate the end of the feeding period. (C) Fasting blood insulin was measured at the end of 5 week feeding period. (D) Liver total cholesterol, (E) free cholesterol and (F) triglycerides were measured at the end of the 5 week feeding period. Results are expressed as the mean \pm SEM $(n=8)$. Different subscript letters indicate statistical significance $(P<0.05)$. 
A
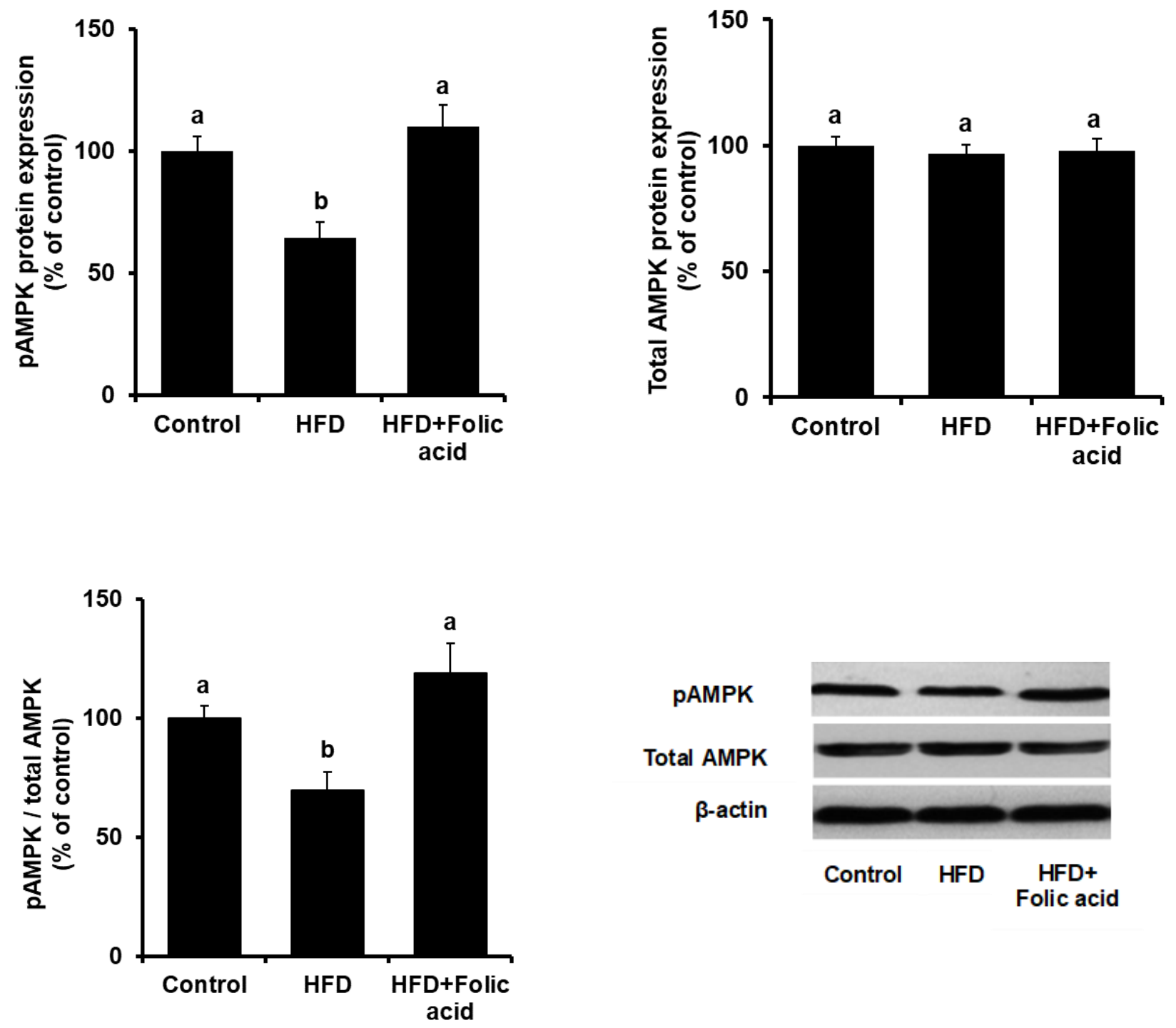

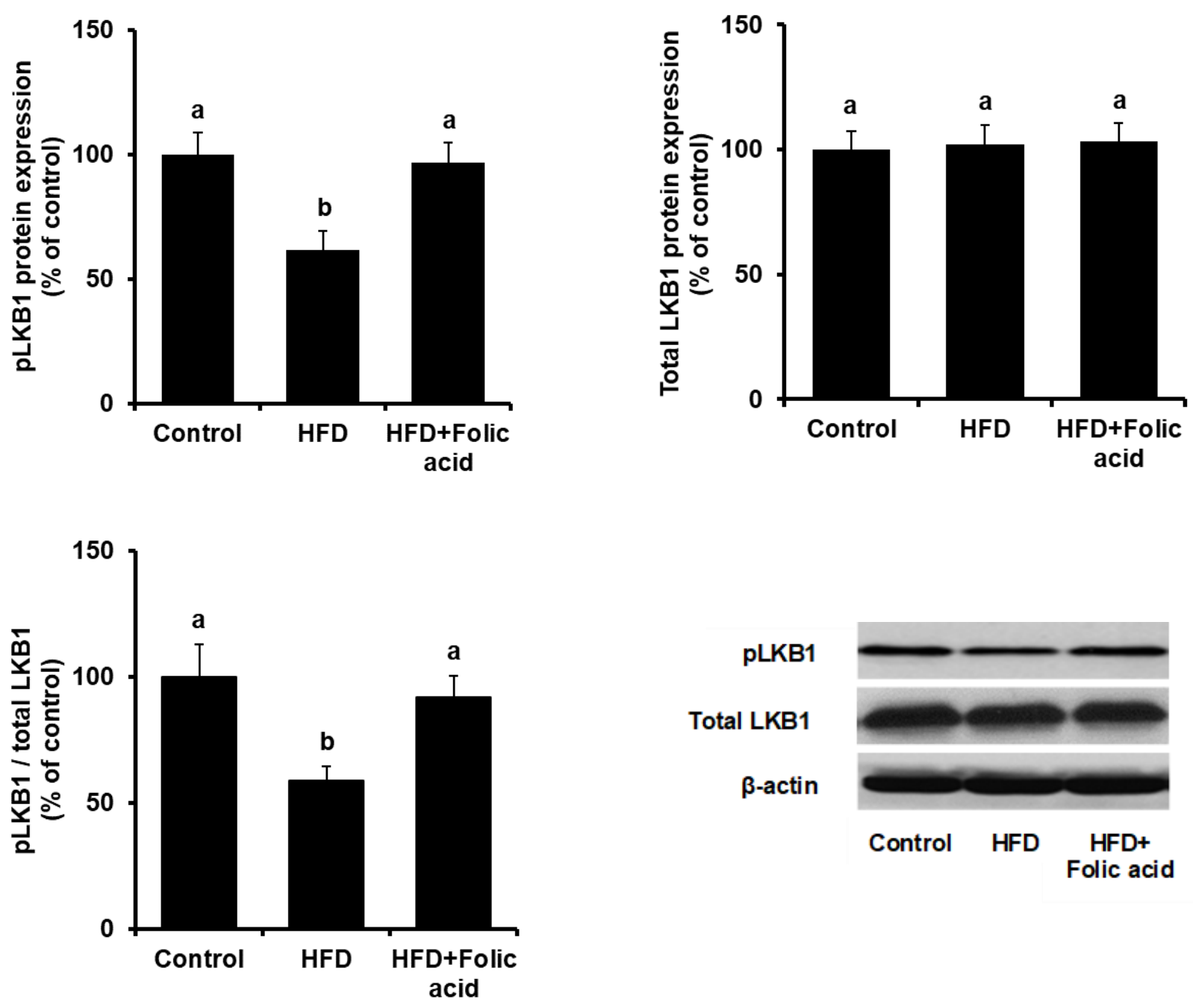

Figure 3.14 AMPK and LKB1 phosphorylation in mouse liver

Mice were fed a control diet, a high-fat diet (HFD) or a high-fat diet supplemented with folic acid (HFD+Folic acid) for 5 weeks. Western immunoblotting analysis was performed to measure the protein levels of phosphorylated AMPK (pAMPK), total AMPK, phosphorylated LKB1 (pLKB1), total LKB1 and $\beta$-actin in the liver. Results are depicted as (A) pAMPK, total AMPK, and ratio of pAMPK to total AMPK, (B) pLKB1, total LKB1, and ratio of pLKB1 to total LKB1. Results are expressed as the mean \pm SEM $(n=8)$. Different subscript letters indicate statistical significance $(P<0.05)$. 
A
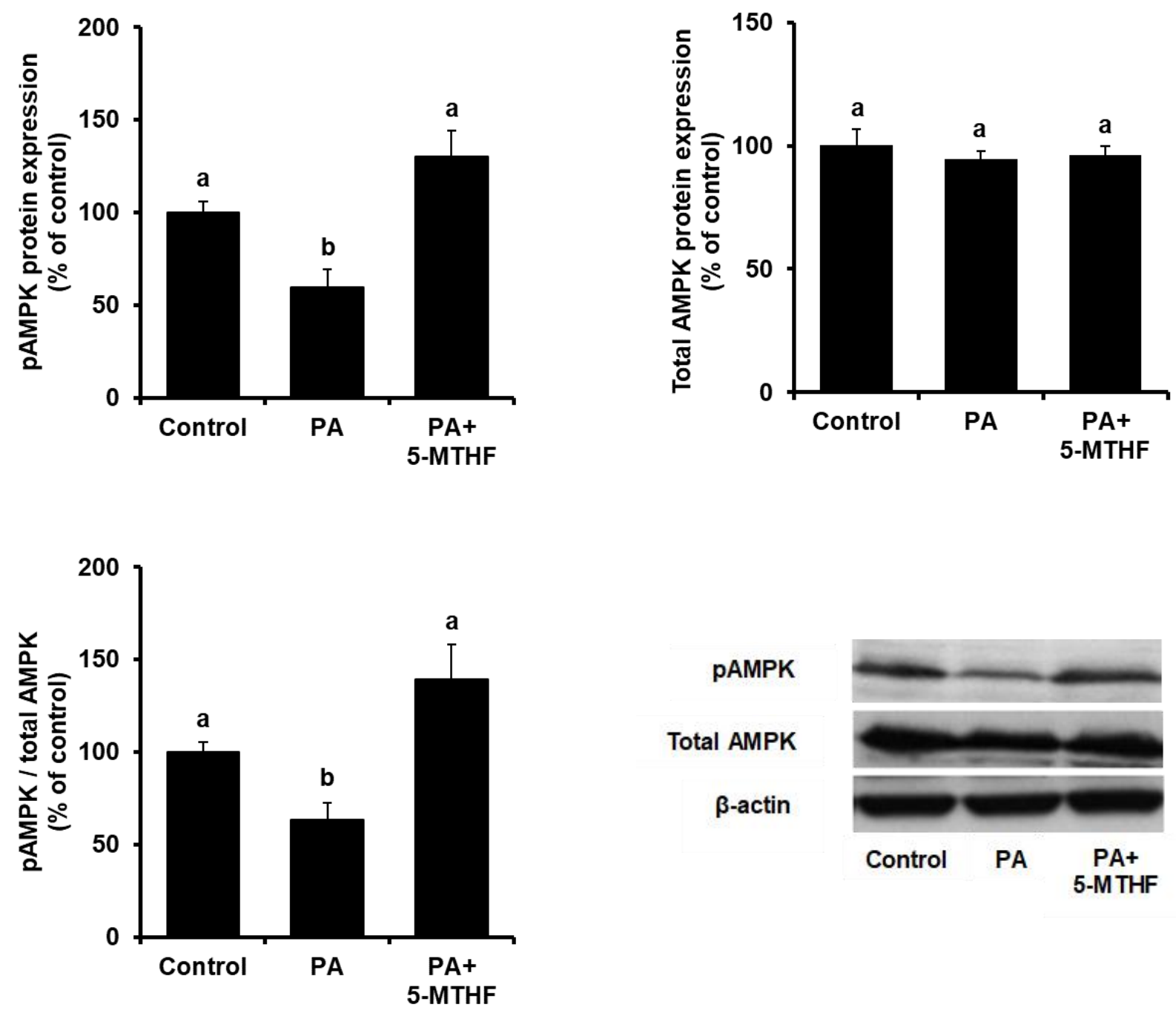

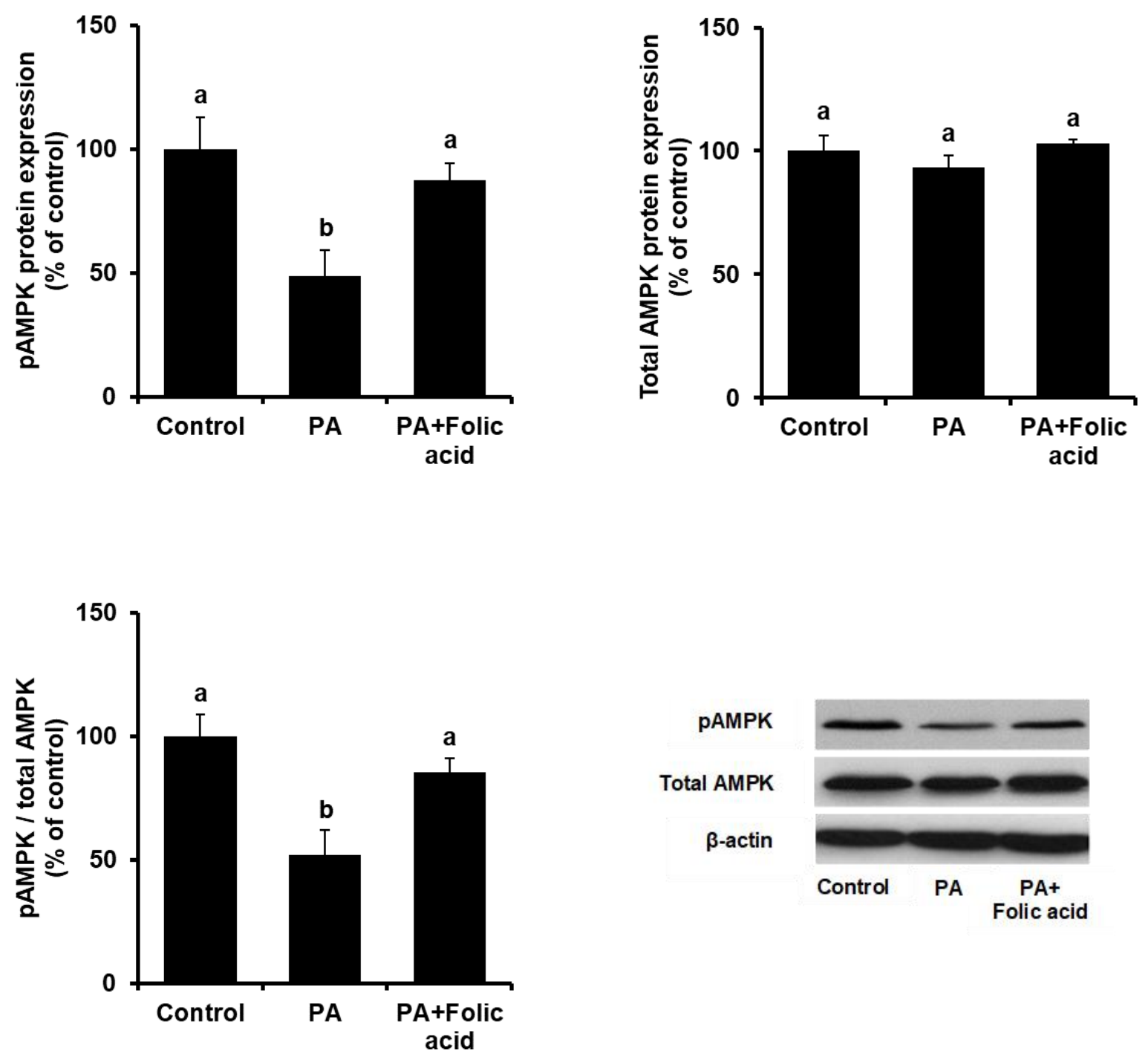
C
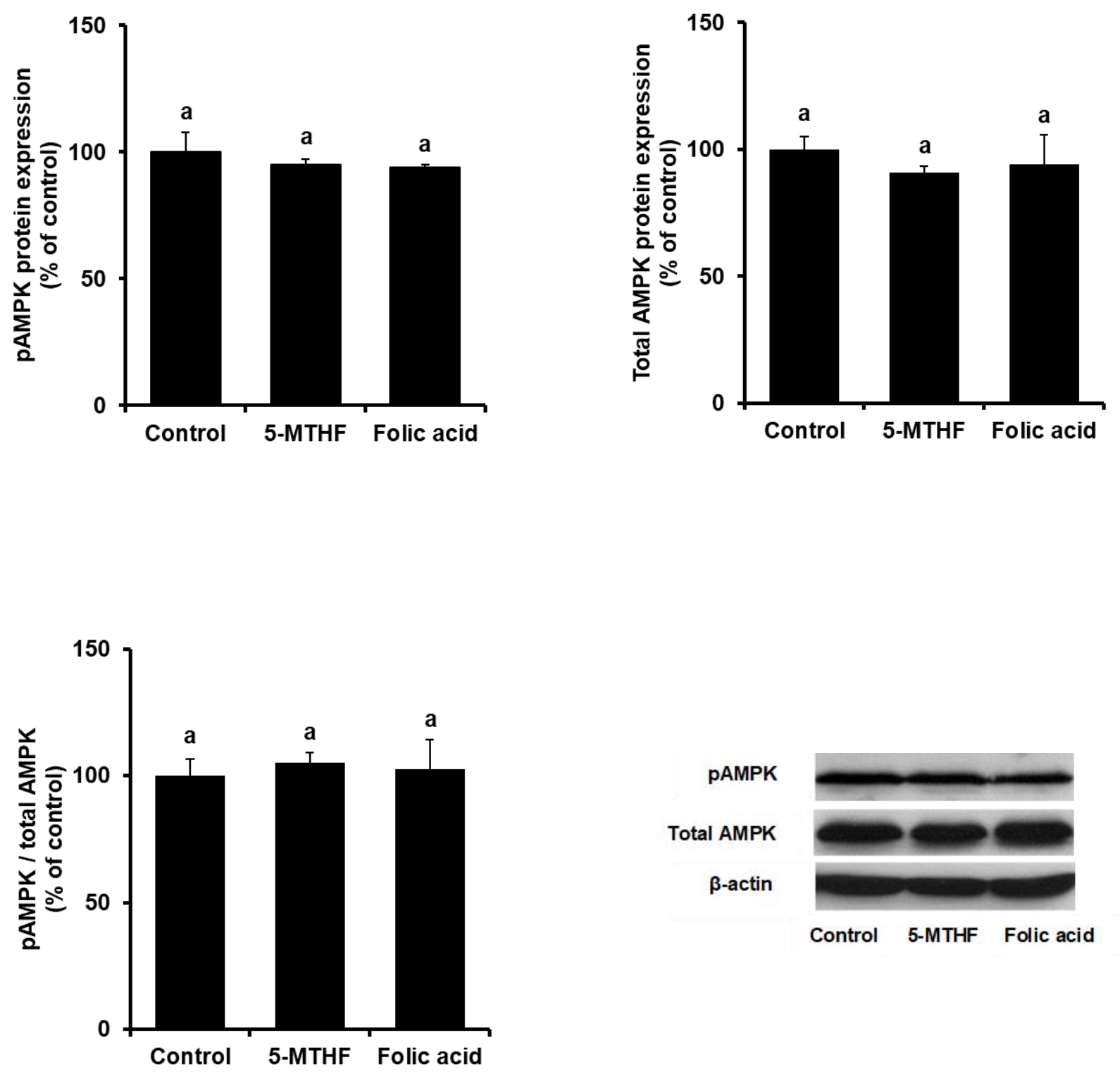

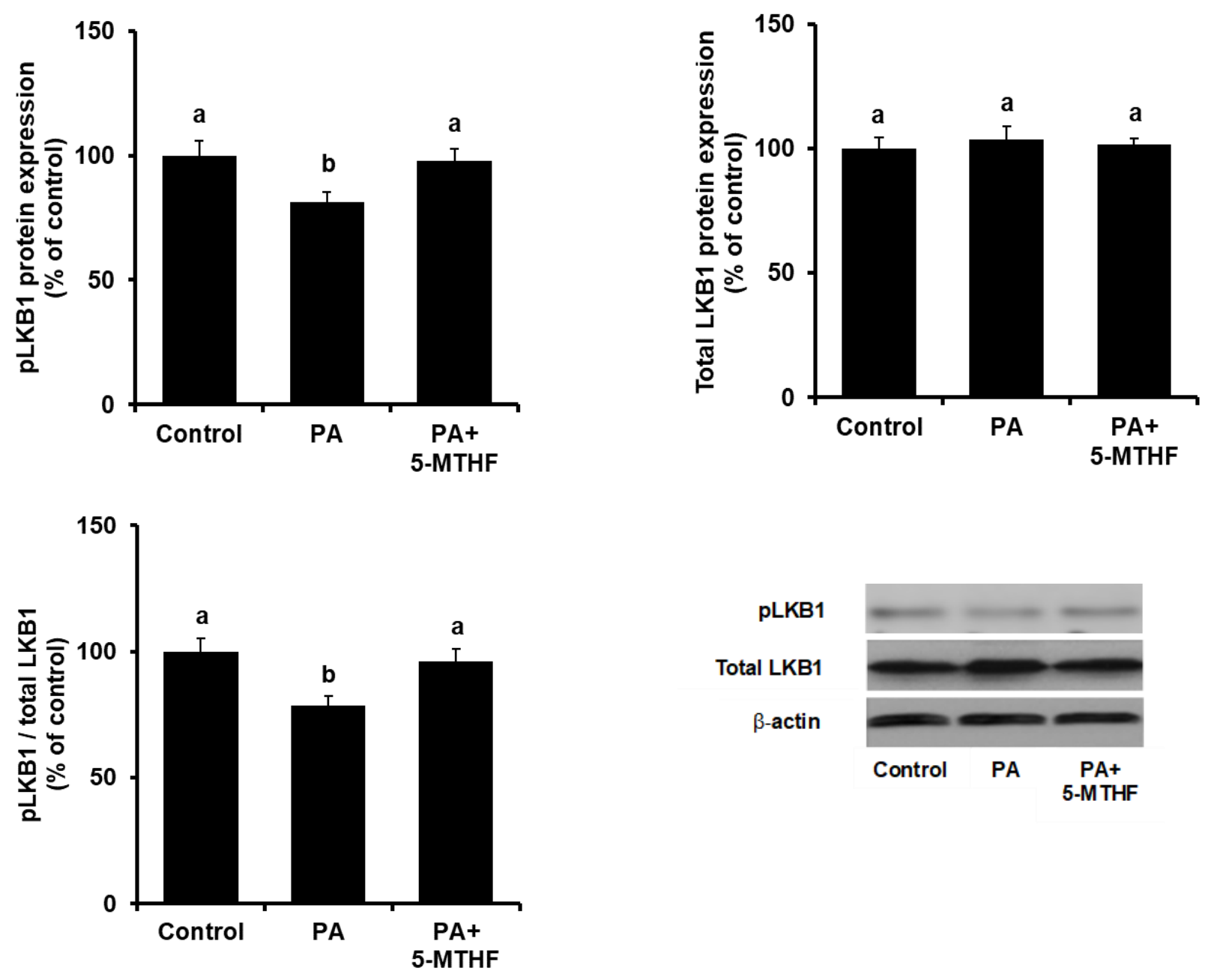

Figure 3.15. AMPK and LKB1 phosphorylation in HepG2 cells

HepG2 cells were incubated in the absence (control) or presence of palmitic acid $(0.3 \mathrm{mM})$ with or without 5-methyltetrahydrofolate $(1 \mu \mathrm{g} / \mathrm{ml}, \mathrm{PA}+5-\mathrm{MTHF})$ or folic acid $(1 \mu \mathrm{g} / \mathrm{ml}, \mathrm{PA}+$ Folic acid) for 16 hours. Control cells were also incubated in the absence or presence of 5-MTHF and folic acid treatment for 16 hours. Western immunoblotting analysis was performed to measure the protein levels of phosphorylated AMPK (pAMPK), total AMPK, phosphorylated LKB1 (pLKB1), total LKB1 in HepG2 cells. $\beta$-actin was measured and used as a loading control. Results are depicted as (A) pAMPK, total AMPK, ratio of pAMPK to total AMPK in palmitic acid-treated cells with or without 5-MTHF treatment; (B) pAMPK, total AMPK, ratio of pAMPK to total AMPK in palmitic acid-treated cells with or without folic acid treatment; (C) pAMPK, total AMPK, ratio of pAMPK to total AMPK in cells incubated with 5-MTHF or folic acid in the absence of palmitic acid treatment; (D) pLKB1, total LKB1, ratio of pLKB1 to total LKB1 in palmitic acid-treated cells. Results are expressed as the mean \pm SEM $(n=4-6)$. Different subscript letters indicate statistical significance $(P<0.05)$. 

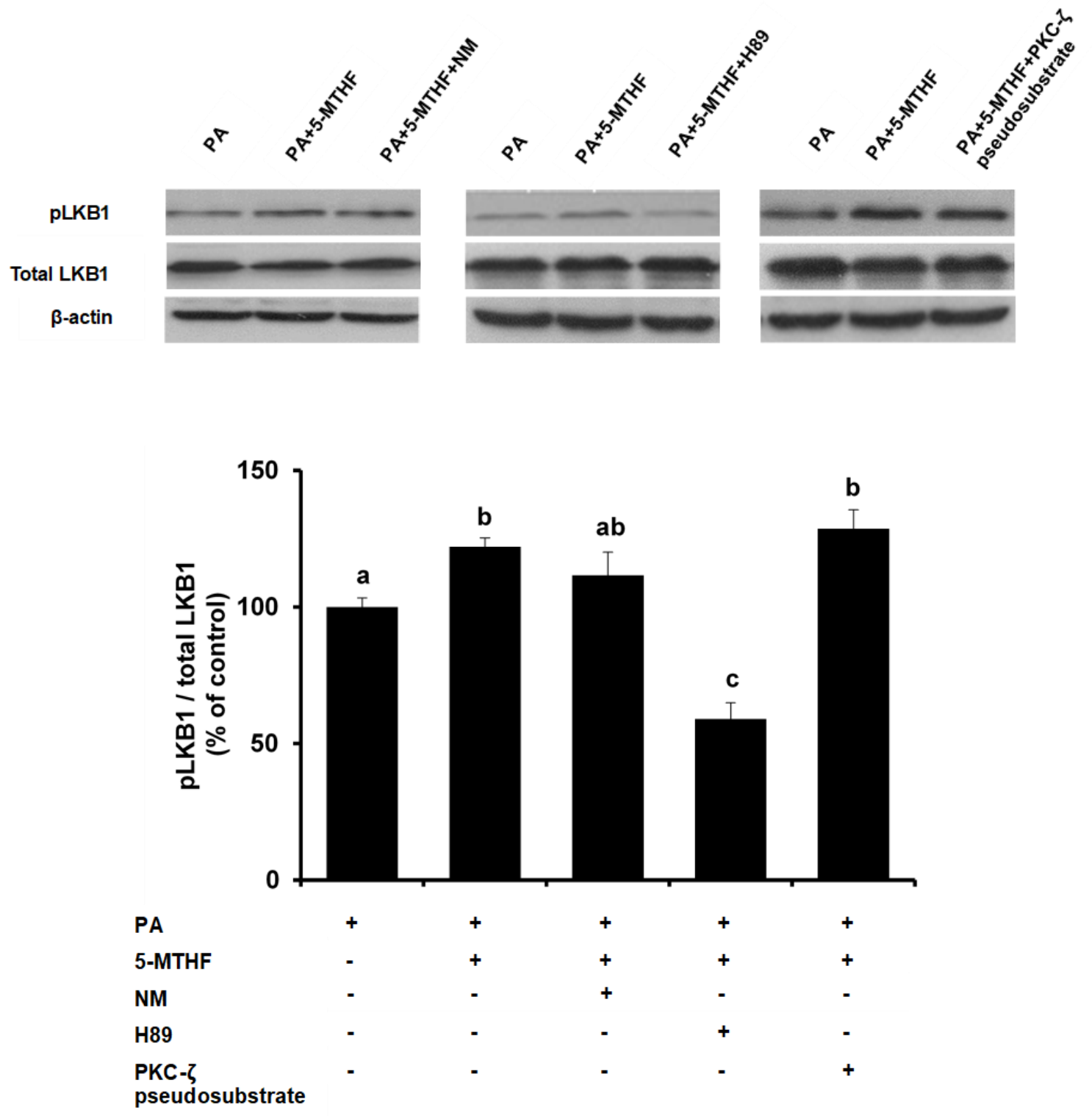

Figure 3.16. Regulation of LKB1 by folic acid in HepG2 cells

HepG2 cells were incubated with palmitic acid $(0.3 \mathrm{mM})$ in the absence or presence of 5 methyltetrahydrofolate $(1 \mu \mathrm{g} / \mathrm{ml}$, 5-MTHF) for 16 hours. In some sets of experiments, nicotinamide $(10 \mathrm{mM}, \mathrm{NM}), \mathrm{H} 89(10 \mu \mathrm{M})$ or PKC- $\zeta$ pseudosubstrate $(1 \mu \mathrm{M})$ were added to the culture medium. Western immunoblotting analysis was performed to measure the protein levels of phosphorylated LKB1 (pLKB1), total LKB1 and $\beta$-actin in cultured cells. Results are depicted as a ratio of pLKB1 to total LKB1 and expressed as the mean \pm SEM $(n=4-6)$. Different subscript letters indicate statistical significance $(P<0.05)$. 

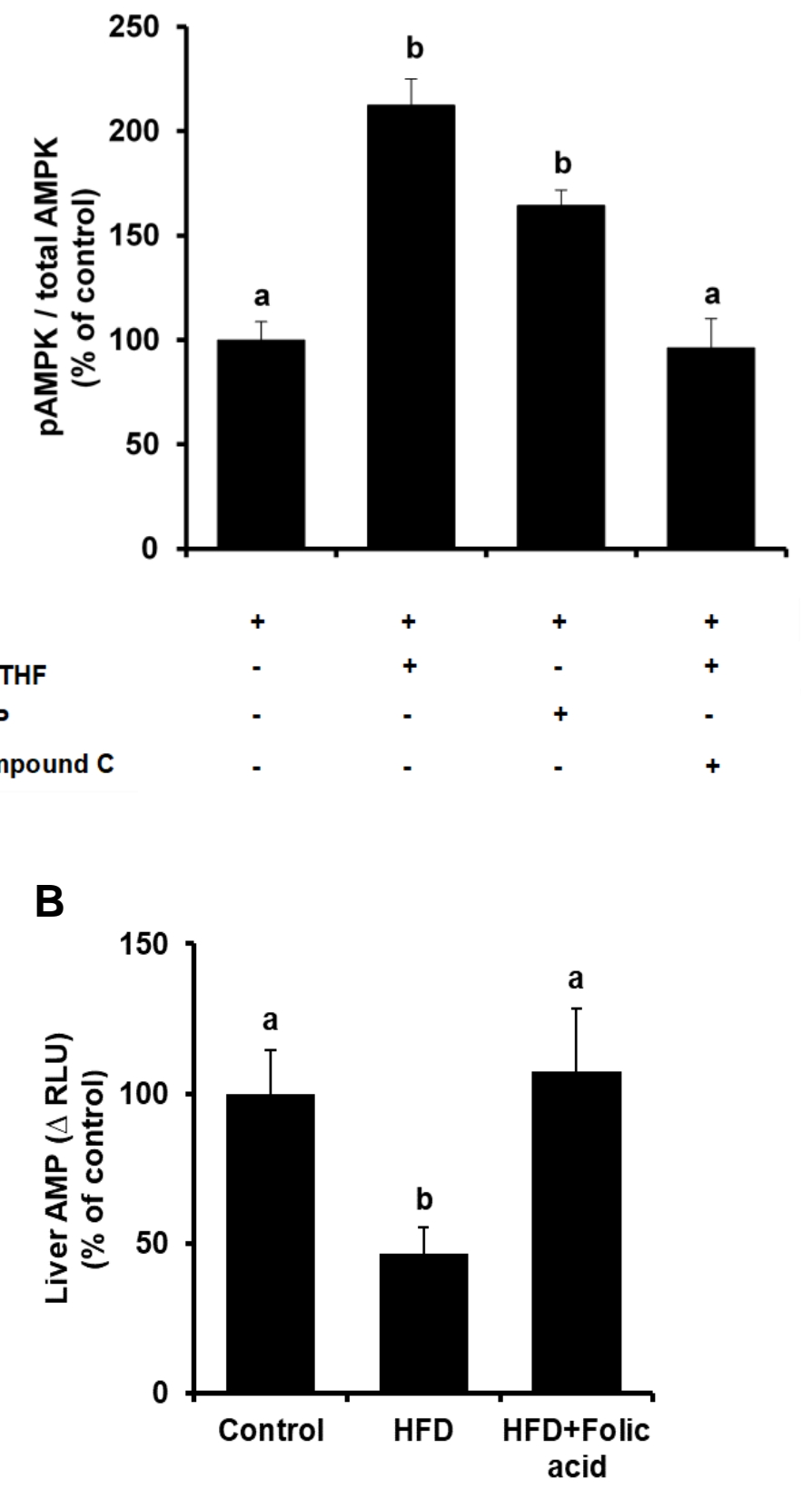

Figure 3.17. AMPK phosphorylation in HepG2 cells and AMP levels in mouse liver

(A) HepG2 cells were incubated with palmitic acid $(0.3 \mathrm{mM})$ in the absence or presence of 5methyltetrahydrofolate $(1 \mu \mathrm{g} / \mathrm{ml}, 5-\mathrm{MTHF})$ for 16 hours. In one set of experiments, compound C $(10 \mu \mathrm{M})$ or AMP $(100 \mu \mathrm{M})$ was added to the culture medium. Western immunoblotting analysis was performed to measure the protein levels of phosphorylated AMPK (pAMPK), total AMPK and $\beta$-actin in cultured cells. Results are depicted as a ratio of pAMPK to total AMPK and expressed as the mean \pm SEM ( $n=4-6)$. (B) Mice were fed a control diet, a high-fat diet (HFD) or a high-fat diet supplemented with folic acid (HFD+Folic acid) for 5 weeks. AMP levels in the liver were measured and results were expressed as changes in relative luminescence units $(\triangle \mathrm{RLU})$. Results are expressed as the mean \pm SEM $(n=6)$. Different subscript letters indicate statistical significance $(P<0.05)$. 


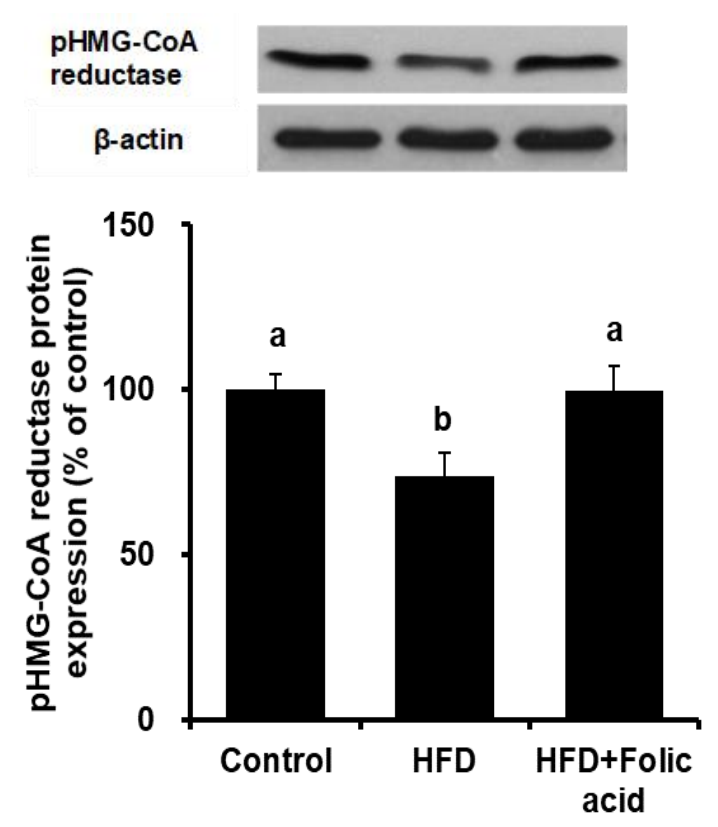

B

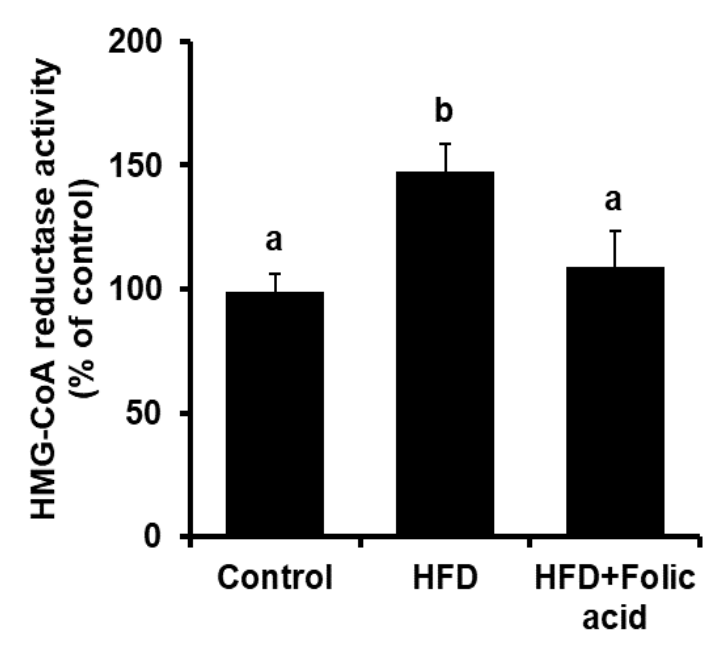

Figure 3.18. Determination of HMG-CoA reductase protein and enzyme activity in mouse liver

Mice were fed a control diet, a high-fat diet (HFD) or a high-fat diet supplemented with folic acid (HFD+Folic acid) for 5 weeks. (A) Phosphorylated HMG-CoA reductase (pHMG-CoA reductase) and $\beta$-actin in the liver were determined by Western immunoblotting analysis. (B) HMG-CoA reductase enzyme activity was measured. Results are expressed as the mean \pm SEM $(\mathrm{n}=4)$. Different subscript letters indicate statistical significance $(P<0.05)$. 


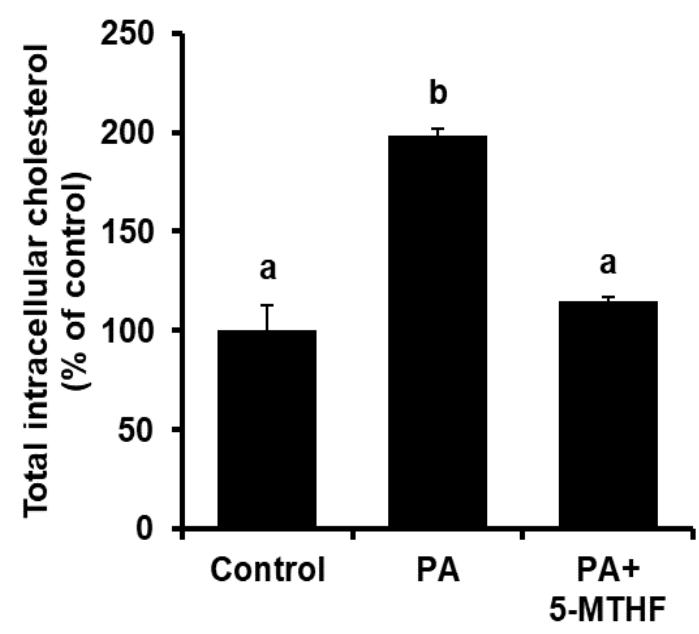

Figure 3.19. Determination of cholesterol in HepG2 cells

HepG2 cells were incubated in the absence or presence of palmitic acid $(0.3 \mathrm{mM})$ with or without 5-methyltetrahydrofolate (5-MTHF, $1 \mu \mathrm{g} / \mathrm{ml}$ ) for 24 hours. Intracellular total cholesterol was measured. Results are expressed as mean \pm SEM $(n=6)$. Different subscript letters indicate statistical significance $(P<0.05)$. 


\subsubsection{Discussion}

AMPK plays a central role in sensing energy levels to favorably modulate hepatic metabolism. With widespread control over a variety of metabolic cascades, AMPK regulation might be important in NAFLD, in which both energy homeostasis and metabolic function are perturbed (Rui 2014). The novel findings presented in this study are that folic acid supplementation effectively restores AMPK activity in the liver of high-fat diet fed mice through: 1) the elevation of AMP levels, and 2) the activation of its upstream kinase LKB1. Such effects of folic acid supplementation are associated with reduced blood glucose and hepatic cholesterol levels in high-fat diet fed mice.

AMP, an allosteric activator of AMPK, mediates its effect via inducing a conformational change in AMPK and subsequently promotes its phosphorylation by upstream kinases (Hardie et al. 2012, Gowans et al. 2013). Although studies have demonstrated that high-fat diet feeding in rodents leads to AMPK inactivation in the liver (Yoneda et al. 2010, Pu et al. 2012), hepatic AMP levels were not reported in these studies. For the first time, the present study demonstrated that hepatic AMP levels were significantly lower in mice fed a high-fat diet than those fed a control diet. Folic acid supplementation effectively increased hepatic AMP levels in high-fat diet fed mice to the control level. Folic acid plays an important role in nucleotide biosynthesis (Lucock 2000). It is plausible that folic acid supplementation may contribute to increased formation of AMP during high-fat diet feeding and therefore, stimulate AMPK activation in the liver. To investigate whether the restoration of hepatic AMP levels contributed to folic acidmediated AMPK activation, experiments were carried out in cultured hepatocytes. While incubation of cells with palmitic acid significantly reduced AMPK phosphorylation (inactivation), its phosphorylation status was restored with 5-MTHF treatment. Furthermore, 
incubation of hepatocytes with AMP was able to reverse the inhibitory effect of palmitic acid on AMPK phosphorylation, indicating that increased AMP availability could stimulate AMPK activation. Taken together, these results suggested that AMPK activation by folic acid might be mediated, in part, through the elevation of hepatic AMP levels in high-fat diet fed mice.

In addition to allosteric activation by AMP, the upstream kinase LKB1 also plays a crucial role in AMPK activation through direct phosphorylation at Thr-172 of AMPK (Woods et al. 2003). In the present study, high-fat diet feeding led to a significant decrease in LKB1 phosphorylation (inactivation) in the liver. By contrast, folic acid supplementation restored hepatic LKB1 phosphorylation status in these animals. Consistent with the in vivo findings, 5-MTHF and folic acid treatment restored AMPK phosphorylation (activation) in palmitic acid-treated cells. Treatment with 5-MTHF in HepG2 cells also stimulated LKB1 phosphorylation (activation). Upstream kinases, namely, PKA and PKC- $\zeta$ have been implicated in the phosphorylation of LKB1 (Collins et al. 2000, Xie et al. 2008). In the present study, incubation of cells with PKA inhibitor (H89) abolished 5-MTHF-induced LKB1 phosphorylation, while inhibition of PKC- $\zeta$ with PKC- $\zeta$ pseudosubstrate did not appear to affect LKB1 phosphorylation. Although deacetylation of LKB1 by SIRT1 is also regarded as another mechanism for LKB1 activation (Hou et al. 2008, Lan et al. 2008), inhibition of SIRT1 by nicotinamide did not appear to significantly affect 5-MTHF-induced LKB1 phosphorylation in HepG2 cells. The effects of 5MTHF observed in palmitic acid-treated HepG2 cells were in line with the findings obtained from the mice fed a high-fat diet supplemented with folic acid. HepG2 cells resemble many characteristics of hepatocytes and have been used as a valuable cell model in metabolic studies including regulation of AMPK (Hou et al. 2008). Similar to primary human hepatocytes, HepG2 cells express the major enzymes that are involved in folate metabolism such as dihydrofolate 
reductase (DHFR) and methylene tetrahydrofolate reductase (MTHFR) (Chango et al. 2009). However, HepG2 cells are transformed cells in which the expression of certain genes may be different from that in primary human hepatocytes. For example, HepG2 cells express lower level of some key enzymes involved in one-carbon or methyl group metabolism such as phosphatidylethanolamine $\mathrm{N}$-methyltransferase (PEMT). PEMT is an enzyme involved in the conversion of phosphatidylethanolamine to phosphatidylcholine in the liver. Reduced expression of this enzyme affects phosphatidylcholine synthesis, which, in turn, can impair hepatic assembly and secretion of VLDL (Cui et al. 1994, Ling et al. 2013). Taken together, results from the present study suggested that PKA might be involved in folic acid-induced LKB1 phosphorylation, which, in turn, contributed to the restoration of hepatic AMPK activation in high-fat diet fed mice supplemented with folic acid. It is intriguing that the insulin sensitizing agent metformin, which is commonly used for the management of diabetes and other metabolic syndromes, also targets the LKB1-AMPK pathway (Despres 2003, Shaw et al. 2005). The ability of folic acid to regulate AMPK activation through an AMP-LKB1-dependent mechanism suggests that it may also have a therapeutic application in metabolic disorders such as high-fat diet-induced NAFLD.

AMPK plays a key role in regulating glucose and cholesterol metabolism (Viollet et al. 2009, Canto et al. 2010), both of which are dysregulated during NAFLD (Marchesini et al. 2003, Min et al. 2012). In the present study, folic acid supplementation effectively attenuated the increase in fasting blood glucose levels in mice fed a high-fat diet but did not significantly reduce blood insulin levels. It is plausible that the favorable effect of folic acid on blood glucose levels might be mediated, in part, by improving hepatic insulin sensitivity. On the other hand, increased cholesterol biosynthesis has been observed in patients with NAFLD (Simonen et al. 2011). Our 
recent study has shown that high-fat diet feeding activates HMG-CoA reductase, which may contribute to hepatic cholesterol accumulation in NAFLD (Wu et al. 2013). The HMG-CoA reductase activity can be regulated by phosphorylation (inactivation) and dephosphorylation (activation) of the enzyme (Clarke et al. 1990). AMPK is the major upstream kinase responsible for the phosphorylation of HMG-CoA reductase (Omkumar et al. 1994, Jurevics et al. 2000). In the present study, folic acid supplementation reduced hepatic cholesterol levels in mice fed a high-fat diet. Such an effect was associated with increased phosphorylation of HMG-CoA reductase and decreased reductase enzyme activity. In addition, 5-MTHF reduced total cholesterol levels in palmitic acid-treated hepatocytes. Taken together, these results suggested that restoration of AMPK activation by folic acid might contribute to the improvement of glucose and cholesterol metabolism during high-fat diet feeding.

It is important to recognize the differences in folate metabolism between rodents and humans. Folate in the human diet consists of natural folates (generally present in polyglutamated form) and synthetic folic acid (monoglutamated form used for dietary fortification and supplements) while purified rodent diets are supplemented with synthetic folic acid. Folic acid is readily absorbed in the intestine via folate receptors. On the other hand, absorption of natural folates in the diet is less efficient since these folates must be hydrolyzed to a monoglutamate prior to intestinal absorption (Pietrzik et al. 2010, Visentin et al. 2014). Upon absorption, folic acid undergoes biotransformation to become the biologically active form of folate, namely, tetrahydrofolate (THF), a precursor of 5-MTHF. Dihydrofolate reductase (DHFR) catalyzes the reduction of folic acid to dihydrofolate (DHF) and then to THF. The activity of DHFR in human liver is low compared to the rat liver (Bailey et al. 2009). In the present study, mice fed a high-fat diet were supplemented with $26 \mathrm{mg} / \mathrm{kg}$ diet folic acid, a pharmacological dose which was 10 -fold 
higher than the basal level in the rodent diet. Caution should be made when findings are translated from rodents to humans as rodents can efficiently metabolize folic acid and may be able to tolerate higher doses of folic acid as compared to humans. A proper human clinical trial should be conducted to ascertain the optimal dose for the use of folic acid as a therapy for metabolic disorders. Another limitation of the present study was that we only investigated whether folic acid supplementation could restore hepatic AMPK activation that was altered by high-fat diet feeding. Future studies are warranted to investigate whether supplementation of folic acid at various doses affects hepatic metabolism under physiological conditions.

The present study demonstrates, for the first time, that folic acid supplementation during high-fat diet feeding can restore AMPK activation in the liver through the elevation of AMP levels and phosphorylation of LKB1. Restoration of AMPK function may contribute to the improvement of glucose and cholesterol metabolism that are impaired by high-fat diet consumption. Further studies are warranted to investigate whether the regulation of AMPK activation by folic acid is beneficial in metabolic disorders such as NAFLD. 


\section{STUDY 3 - MANUSCRIPT 3}

3.3. Folic acid supplementation attenuates chronic hepatic inflammation in high-fat diet fed mice

V. Sid, Y. Shang, Y.L. Siow, S. Madduma Hewage, J.D. House, K. O. Lipids. 53 (7):709-716 (2018) 


\subsubsection{Abstract}

Non-alcoholic fatty liver disease (NAFLD) is the most common form of chronic liver disease worldwide. Hepatic inflammation is an important pathogenic mediator of NAFLD. There is currently no pharmacological agent approved for the treatment of NAFLD. Folic acid is a watersoluble B vitamin that has been shown to have lipid-lowering and antioxidant effects. The objective of this study was to investigate the effect of folic acid supplementation on hepatic inflammation and to identify the underlying mechanisms. Male C57BL/6J mice were fed a control diet ( $10 \% \mathrm{kcal}$ fat), a high-fat diet $(60 \% \mathrm{kcal}$ fat), or a high-fat diet supplemented with folic acid (26mg/kg diet) for 8 weeks. High-fat diet feeding led to increased body mass gain, lipid accumulation, activation of transcription factor NF- $\mathrm{kB}$, and elevation of inflammatory cytokine gene expression in the liver. Folic acid supplementation attenuated hepatic lipid accumulation and aggregation of inflammatory foci induced by high-fat diet feeding. This was associated with a significant reduction of NF- $\mathrm{KB}$ activation and inflammatory cytokine expression. These results suggest that the hepatoprotective effect of folic acid in NAFLD may be attributed, in part, to its anti-inflammatory action. 


\subsubsection{Introduction}

Non-alcoholic fatty liver disease (NAFLD) is a rapidly growing health problem that currently affects over 1 billion people worldwide (Loomba et al. 2013). NAFLD covers a broad spectrum of liver disorders that ranges from steatosis (fatty liver) to non-alcoholic steatohepatitis (NASH) and cirrhosis in its advanced stages (Cohen et al. 2011). In contrast to steatosis, NASH is characterized by lipid accumulation, hepatocyte injury, inflammation and fibrosis (Kleiner et al. 2005, Cohen et al. 2011). Patients with obesity and type 2 diabetes are highly susceptible to the development of NAFLD (Ahmed 2015). Enhanced consumption of high-fat diets, particularly those enriched in saturated fats, induces lipid accumulation and low-grade chronic inflammation in the liver (Leamy et al. 2013, Wu et al. 2013).

Hepatic inflammation is an important pathogenic mediator of NAFLD, and is characterized histologically by the presence of inflammatory foci, which is comprised of clusters of hepatocytes and resident liver immune cells (kupffer cells) (Kleiner et al. 2012). A prolonged consumption of diets that are high in dietary fats leads to excessive delivery of free fatty acids from adipose tissue to the plasma, which contributes to hepatic lipid accumulation in NAFLD (Fabbrini et al. 2008). High-fat diet consumption in mice leads to significant increases in hepatic triglyceride content and serum free fatty acid concentrations (Cai et al. 2005). Asides from inducing lipid accumulation, exposure to high concentrations of free fatty acids is hepatotoxic and activates inflammatory signaling cascades in the liver (Joshi-Barve et al. 2007). The nuclear factor- $\kappa \mathrm{B}(\mathrm{NF}-\mathrm{\kappa B})$ is a key transcriptional factor that drives the expression of pro-inflammatory cytokines such as tumor necrosis factor- $\alpha$ (TNF- $\alpha$ ) and interleukin-6 (IL-6) (Barnes et al. 1997). It has been shown that hepatic production of pro-inflammatory cytokines and activation of NF$\kappa \mathrm{B}$ are prominently upregulated in the liver of mice fed a high-fat diet (Cai et al. 2005), as well 
as in patients with NAFLD (Ribeiro et al. 2004). High levels of IL-6 and TNF- $\alpha$ facilitate recruitment of macrophages and neutrophils to the liver and can result in a state of chronically unresolved inflammation (Park et al. 2010). Inhibition of NF- $\mathrm{kB}$ signaling can ameliorate the hepatic inflammatory response in mice fed a high-fat diet (Cai et al. 2005).

There is currently no pharmacological agent approved for the treatment of NAFLD (Schuppan et al. 2013, Sid et al. 2017). Folic acid is a water-soluble B vitamin that has been shown to modulate lipid metabolism and oxidative stress (Sarna et al. 2012). It has been suggested that folate deficiency contributes to the development of steatosis (Christensen et al. 2010). Depletion of dietary folates has been shown to be associated with perturbed expression of genes involved in lipid biosynthesis (Champier et al. 2012). Folic acid also exhibits antioxidant functions, attributed to its ability to scavenge reactive oxygen species (ROS), inhibit the activity of ROSgenerating enzymes and restore antioxidant enzyme activity (Gliszczynska-Swiglo et al. 2007, Sarna et al. 2012). We have recently demonstrated that folic acid supplementation effectively alleviates hepatic oxidative stress and reduces lipid accumulation in mice fed by high-fat diet (Sarna et al. 2012, Sid et al. 2015). Folic acid can also reduce the circulating level of proinflammatory mediators in overweight individuals and in hyperhomocysteinemic patients (Wang et al. 2005, Solini et al. 2006). However, the anti-inflammatory effect of folic acid in the context of NAFLD is not clear. In the present study, we investigated the effects of folic acid supplementation on hepatic inflammation in high-fat diet fed mice and the mechanisms involved. 


\subsubsection{Materials and Methods}

i) Animal model

Male C57BL/6J mice aged 6 weeks (Central Animal Care Services, University of Manitoba, Winnipeg, MB, CAN) were fed a control diet (D12450B; 10\% kcal fat), a high-fat diet (D12492; $60 \% \mathrm{kcal}$ fat $)$ or a high-fat diet with folic acid supplementation $(26 \mathrm{mg} / \mathrm{kg}$ diet $)$ for 8 weeks. In a pilot study, the dose of folic acid $(26 \mathrm{mg} / \mathrm{kg})$ was found to be the lowest dose that had glucose lowering effects. Diets were prepared by Research Diets, Brunswick, NJ and formulated based on their nutrient to calorie ratios. The diet composition is listed in Table 3. Mice were maintained on a 12-hour light/12-hour dark cycle with free access to food and water. There was no significant difference in the average caloric intake between the control and high-fat diet fed mice $(11.02 \pm 0.02$ vs $11.88 \pm 1.27 \mathrm{kcal} / \mathrm{mouse} /$ day) (Shang et al. 2017). Average caloric intake by mice fed a high-fat diet with folic acid supplementation was similar to the high-fat diet fed group $(11.88 \pm 1.27$ vs. $11.68 \pm 0.76 \mathrm{kcal} / \mathrm{mouse} /$ day $)$. Average food intake per day was $2.83 \pm 0.04$ $\mathrm{g} /$ day for mice fed a control diet, $2.27 \pm 0.24 \mathrm{~g} /$ day for mice fed a high-fat diet, and $2.42 \pm 0.21$ g/day for mice fed a high-fat diet with folic acid supplementation. The body mass of mice was recorded at the beginning and the end of the experimental period. Lipids in the liver tissue were extracted according to the Folch method (Folch et al. 1957). The levels of triacylglycerols in the liver were determined by using an enzymatic kit (Sekisui Diagnostics, Lexington, MA, USA). All procedures were performed in accordance with the Guide to the Care and Use of Experimental Animals published by the Canadian Council on Animal Care and approved by the University of Manitoba Protocol Management and Review Committee. 
ii) Cell culture

HepG2 cells (human hepatoblastoma cell line HB-8065; American Type Culture Collection, Manassas, VA) were cultured in DMEM (VWR, Mississauga, ON, CAN) supplemented with $10 \% \mathrm{FBS}$ at $37^{\circ} \mathrm{C}$ in a humidified $5 \% \mathrm{CO}_{2}$ atmosphere. HepG2 cells are routinely used as a hepatocyte model for mechanistic studies (Woo et al. 2006). Cells were treated with palmitic acid (Sigma Aldrich, Oakville, ON, CAN), a major saturated fatty acid in the high-fat diet. Palmitic acid was dissolved in 10\% BSA (essentially fatty acid free, Sigma Aldrich, Oakville, ON, CAN) and 5\% ethanol (Wu et al. 2013, Sid et al. 2015). In some experiments, cells were treated with 5-methyltetrahydrofolate (5-MTHF; Sigma Aldrich, Oakville, ON, CAN), palmitic acid-treated the active form of folate, in the presence of palmitic acid. In one set of experiments, cells were incubated with ammonium pyrrolidinedithiocarbamate (PDTC; Sigma Aldrich, Oakville, ON, CAN), a selective inhibitor of $N F-\kappa B$.

iii) Liver histological analysis

A portion of mouse liver was fixed in $10 \%$ neutral buffered formalin overnight and embedded in paraffin. Paraffin-embedded cross sections were prepared on glass slides. Liver sections were deparaffinized and stained with hematoxylin and eosin (H\&E) to examine morphological changes in the liver such as accumulation of lipid vacuoles and inflammatory foci (Woo et al. 2005). Hepatic inflammatory foci are defined as aggregates of inflammatory cells that accumulate in the liver during chronic inflammation (Wu et al. 2009, Kleiner et al. 2012). Images of H\&E liver sections were captured by using an Olympus BX43 light microscope equipped a Q-Color3 camera and were analyzed at 100x and 200x magnification. 
iv) Real-time polymerase chain reaction (PCR) analysis

Total RNAs were isolated from liver tissue and cells with Trizol reagent (Thermo Fisher Scientific, Waltham, MA, USA). RNA ( $2 \mu \mathrm{g})$ was converted to cDNA by reverse transcription. The mRNA expression of IL- 6 and TNF- $\alpha$ was determined by real-time polymerase chain reaction (PCR) analysis using the iQ5 real-time PCR detection system (Bio-Rad, Mississauga, ON, CAN). The real-time PCR reaction mixture consisted of $0.4 \mu \mathrm{M}$ of 5' and 3' primers and $2 \mathrm{ul}$ of cDNA product in iQ-SYBR green supermix (Bio-Rad, Mississauga, ON, CAN). The primer sequences (Thermo Fisher Scientific, Waltham, MA, USA) used in the study were: mouse IL-6, 5'-GACTGATGCTGGTGACAACC-3' (forward), 5'-GCCATTGCACAACTCTTTTC-3' (reverse); mouse TNF- $\alpha$, 5'-GTCCCCAAAGGGATGAGAAG-3' (forward), 5'-

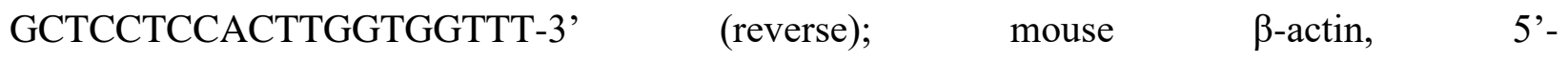
GATCAAGATCATTGCTCCTCCT-3' (forward), 5'-AGGGTGTAAAACGCAGCTCA-3' (reverse); human IL-6, 5'-AGGAGACTTGCCTGGTGAAA-3' (forward), GTCAGGGGTGGTTATTGCAT-3' (reverse); human $\quad \beta$-actin, $\quad 5^{\prime}$ AGATCAAGATCATTGCTCCTCCT (forward), human $\quad \beta$-actin, 5 'GATCCACATCTGCTGGAAGG-3' (reverse). The mRNA expressions of the target genes were normalized against $\beta$-actin values.

v) Electrophoretic mobility shift assay (EMSA)

The DNA binding activity of NF- $\mathrm{BB}$ was measured by EMSA, using the LightShift Chemiluminescent EMSA Kit (Thermo Fisher Scientific, Waltham, MA, USA). In brief, nuclear proteins were prepared from liver tissue, as previously described (Woo et al. 2008, Wu et al. 2009). Nuclear proteins $(2 \mu \mathrm{g})$ were incubated in a reaction mixture containing DNA binding 
buffer, poly (dI-dC) and biotin-end-labeled oligonucleotides containing a consensus sequence specific for the NF- $\mathrm{BB}$ binding site (5'-AGTTGAGGGGACTTCCAGGC-3') (Promega, Madison, WI, USA), according to manufacturer's instructions. The NF-kB oligonucleotide was labelled with biotin at the 3' end using the Biotin 3' End DNA labelling kit (Thermo Fisher Scientific, Waltham, MA, USA). The cold competition assay was performed by incubating nuclear extracts with 200-fold excess of unlabelled oligonucleotides before addition of the biotin labelled probe. Following incubation, reaction mixtures were loaded in a $6 \%$ nondenaturing polyacrylamide gel to facilitate separation of DNA-protein complexes, and transferred to a nylon membrane for detection using the Chemiluminescent Nucleic acid Detection Module Kit (Thermo Fisher Scientific, Waltham, MA, USA).

vi) Statistical Analysis

The data normality and homogeneity of variance was tested using the Bartlett test. If variances were equal across sample groups, the data were subjected to one-way analysis of variance (ANOVA) following Tukey post hoc test. If not, the data was analyzed using the Kruskal-Wallis non-parametric test $(p<0.05)$. 


\subsubsection{Results}

i) Mouse body mass and liver morphology

Mice fed a high-fat diet for 8 weeks exhibited a significant increase in body mass gain compared to mice fed a control diet (Fig. 3.20A). Folic acid supplementation did not change the body mass gain in mice fed a high-fat diet (Fig. 3.20A). Mice fed a high-fat diet displayed elevated levels of triacylglycerols in the liver, which was significantly lowered by folic acid supplementation (Fig. 3.20B). To examine the morphological changes in the liver, mouse liver tissue was stained with H\&E. Mice fed a high-fat diet displayed increased accumulation of lipid vacuoles and deposition of inflammatory foci (Fig. 3.20C). Folic acid supplementation improved liver histology by reducing hepatic lipid vacuoles and inflammatory foci in mice fed a high-fat diet (Fig. 3.20C).

ii) Hepatic cytokine expression and $\mathrm{NF}-\kappa \mathrm{B}$ activation

The mRNA levels of pro-inflammatory cytokines (IL-6, TNF- $\alpha$ ) were significantly elevated in the liver of mice fed a high-fat diet as compared to that in the control group (Fig. 3.21A and 3.21B). Folic acid supplementation effectively lowered the expression of pro-inflammatory cytokines (Fig. 3.21A and 3.21B). The NF- $\mathrm{BB} / \mathrm{DNA}$ binding activity was significantly enhanced in the liver of mice fed a high-fat diet (Fig. 3.21C). Folic acid supplementation significantly reduced hepatic NF- $\kappa \mathrm{B}$ activation in mice fed a high-fat diet (Fig. 3.21C). These results suggested that folic acid supplementation was able to attenuate high-fat diet-induced hepatic

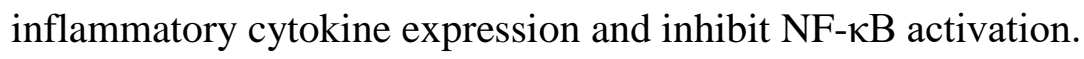


iii) Effect of fatty acid and 5-MTHF in hepatocytes

Palmitic acid is one of the most abundant saturated fatty acids in high-fat diets. The effect of palmitic acid and folic acid on cytokine expression was examined in HepG2 cells. Palmitic acid treatment significantly increased the expression of IL-6 in cells (Fig. 3.22A). This was associated with enhanced NF- $\kappa \mathrm{B} / \mathrm{DNA}$ binding activity (Fig. 3.22B). Activation of NF- $\kappa \mathrm{B}$ was detected in cells incubated with palmitic acid for 15, 30, 60 and 120 minutes (Fig. 3.22B). The highest DNA binding activity of NF- $\kappa \mathrm{B}$ was detected at 15 minutes. To investigate the effect of folic acid on palmitic acid-induced NF- $\mathrm{B}$ activation and cytokine expression, cells were incubated with folic acid (5-MTHF). Treatment of cells with 5-MTHF attenuated palmitic acid-induced elevation of IL-6 gene expression (Fig. 3.22A) as well as NF- $\mathrm{BB}$ activation (Fig. 3.22C). Incubation of cells with an inhibitor of NF- $\mathrm{B}$ (PDTC) not only attenuated palmitic acid-induced NF- $\kappa \mathrm{B}$ activation (Fig. 3.23A) but also reduced IL-6 expression (Fig. 3.23B). 
Table 3. Composition of purified diets: energy, macronutrient and other primary ingredients

\begin{tabular}{|c|c|c|c|}
\hline & $\begin{array}{c}\text { Control } \\
\text { (D12450B) }\end{array}$ & $\begin{array}{c}\text { HFD } \\
\text { (D12492) }\end{array}$ & HFD+Folic acid \\
\hline \multicolumn{4}{|l|}{ Energy content (\% kcal) } \\
\hline Fat & 10 & 60 & 60 \\
\hline Carbohydrate & 70 & 20 & 20 \\
\hline Protein & 20 & 20 & 20 \\
\hline \multicolumn{4}{|l|}{ Fat content $(\mathbf{g} / \mathbf{k g})$} \\
\hline Soybean oil & 24 & 32 & 32 \\
\hline Lard & 19 & 317 & 317 \\
\hline \multicolumn{4}{|l|}{ Carbohydrate (g/kg) } \\
\hline Corn Starch & 299 & 0 & 0 \\
\hline Maltodextrin 10 & 33 & 162 & 162 \\
\hline Sucrose & 332 & 89 & 89 \\
\hline Cellulose, BW200 & 47 & 65 & 65 \\
\hline \multicolumn{4}{|l|}{ Protein (g/kg) } \\
\hline Casein, 80 mesh & 190 & 258 & 258 \\
\hline L-Cystine & 2.84 & 3.88 & 3.88 \\
\hline Mineral Mix, S10026 (g/kg) & 9 & 13 & 13 \\
\hline Vitamin Mix, V10001 (g/kg) & 9 & 13 & 13 \\
\hline Folic acid $(\mathbf{m g} / \mathbf{k g})$ & 2 & 2.6 & 26 \\
\hline Dietary energy (kcal/g) & 3.85 & 5.24 & 5.24 \\
\hline
\end{tabular}


A

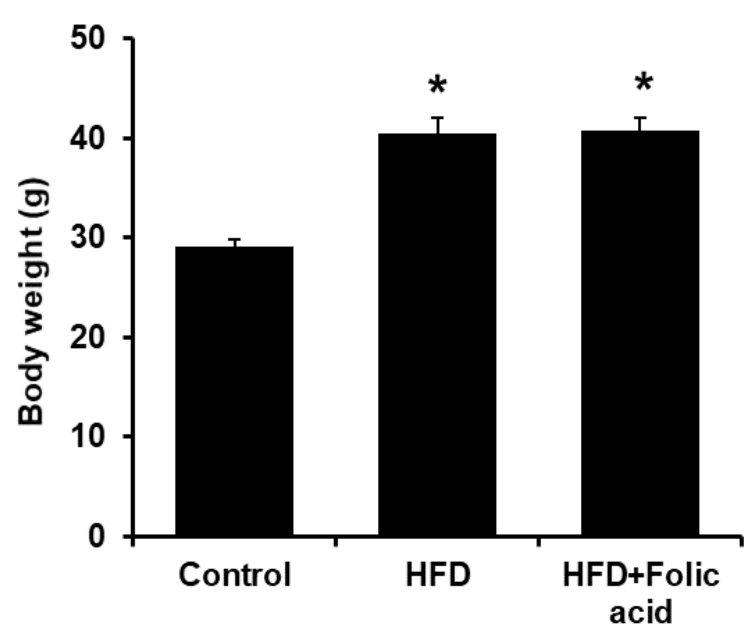

C
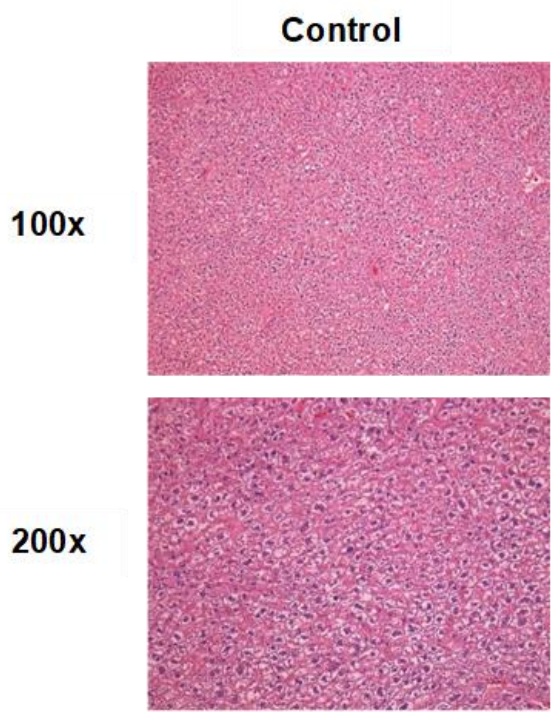

$200 x$
B

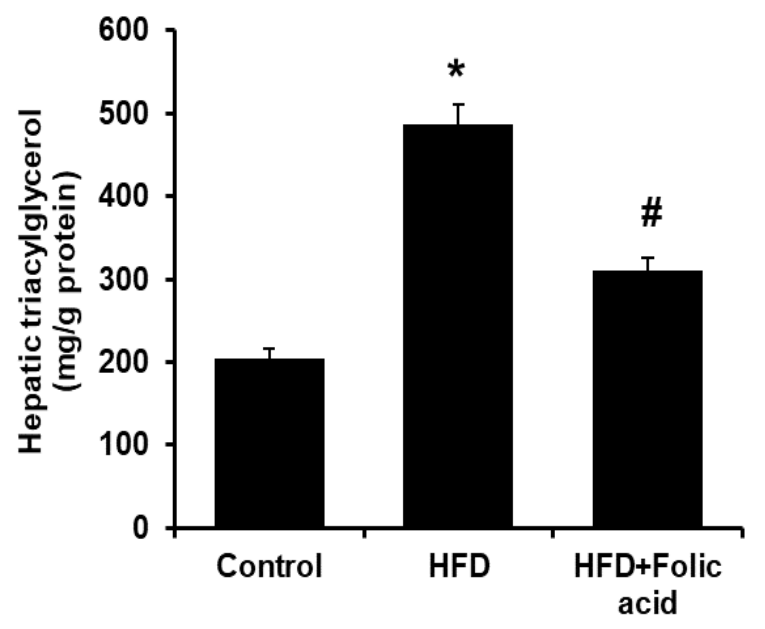

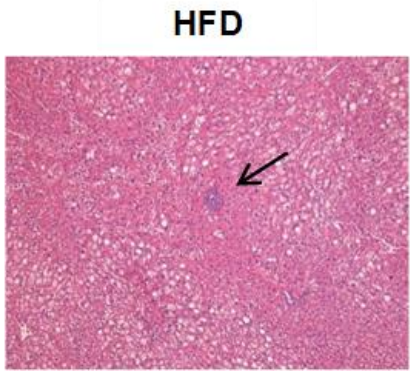

HFD+Folic acid
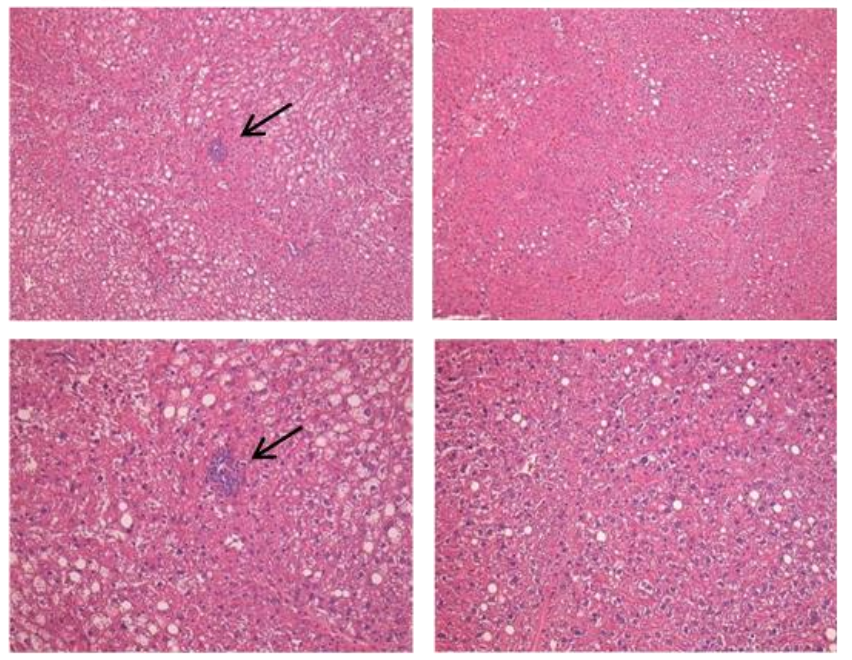

\section{Figure 3.20. Body mass and liver histology}

Mice were fed a control diet, high-fat diet (HFD) or a high-fat diet supplemented with folic acid (HFD + folic acid) for 8 weeks. (A) Body mass and (B) liver triacylglycerol were measured at the end of feeding period. (C) Liver histology was examined by H\&E staining. Arrow points to inflammatory foci. The results are expressed as the means \pm S.E.M $(\mathrm{n}=6) *, p<0.05$ when compared with the value obtained from the control group. \#, $p<0.05$ when compared with the value obtained from HFD group. 
A

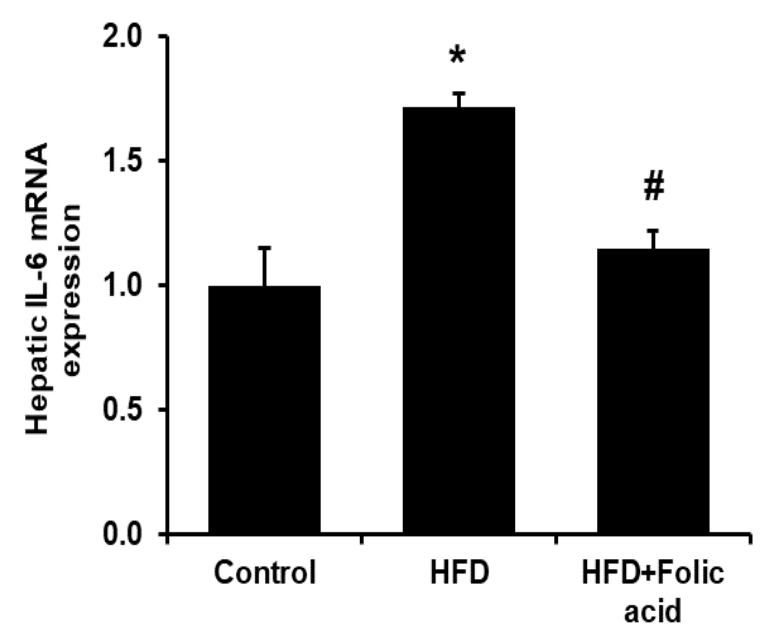

B

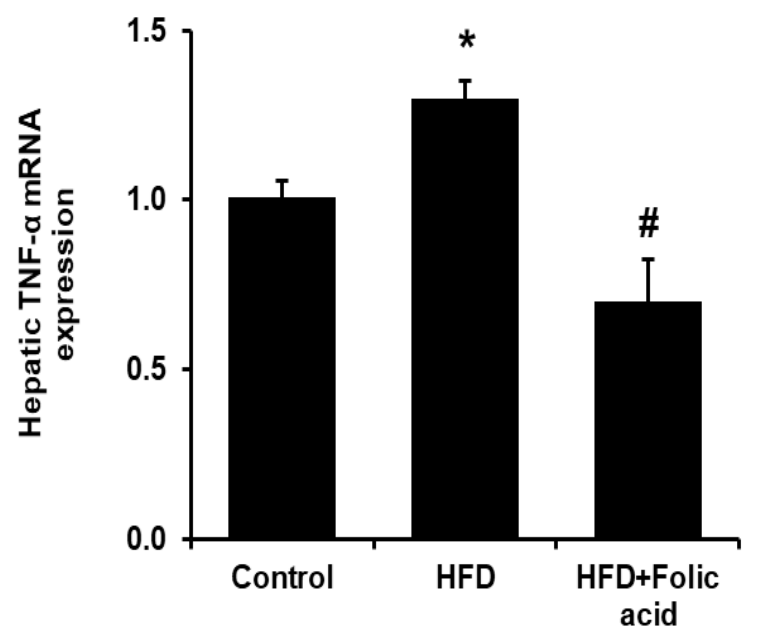

C
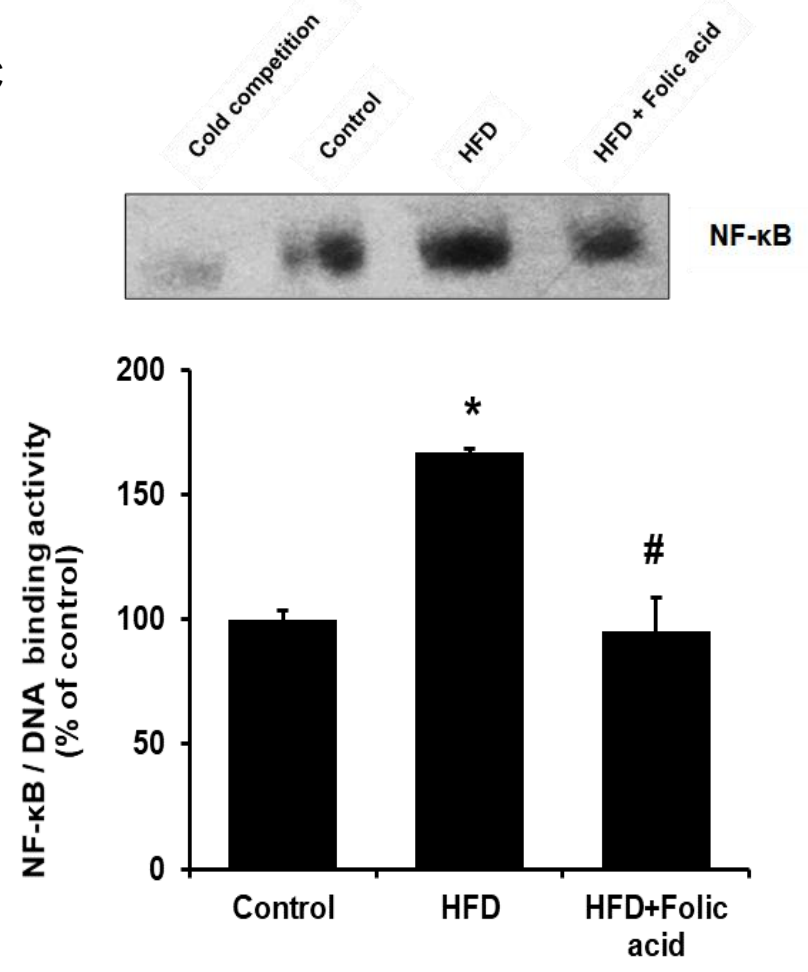

Figure 3.21. Effect of folic acid on inflammatory cytokine expression and NF-кB binding activity in mouse liver

Mice were fed a control diet, high-fat diet (HFD) or a high-fat diet supplemented with folic acid (HFD + folic acid) for 8 weeks. (A) IL-6 and (B) TNF- $\alpha$ mRNA expression was determined by real-time PCR analysis. (C) NF- $\kappa \mathrm{B}$ DNA binding activity in liver tissue was determined by EMSA. The results are expressed as the means \pm S.E.M. $(\mathrm{n}=4-6) .{ }^{*} p<0.05$ when compared with the value obtained from the control group. \#, $p<0.05$ when compared with the value obtained from HFD group. 


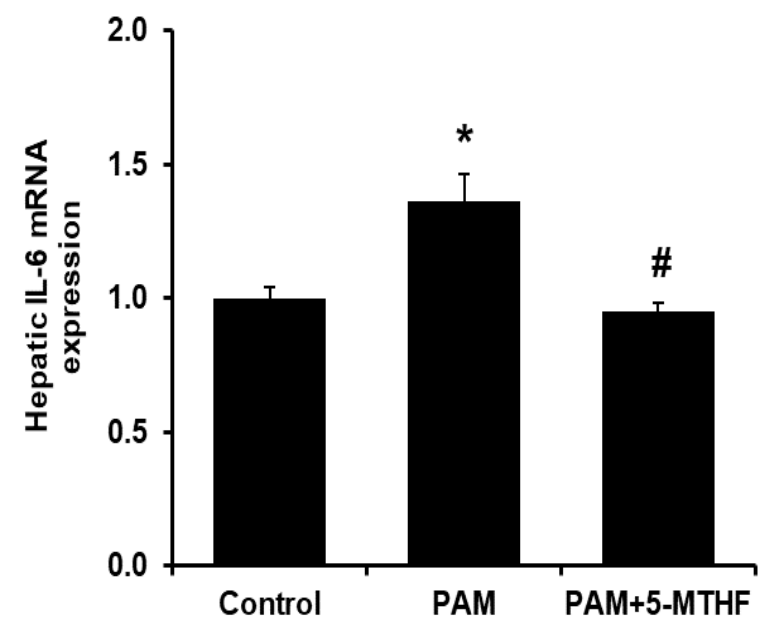

B
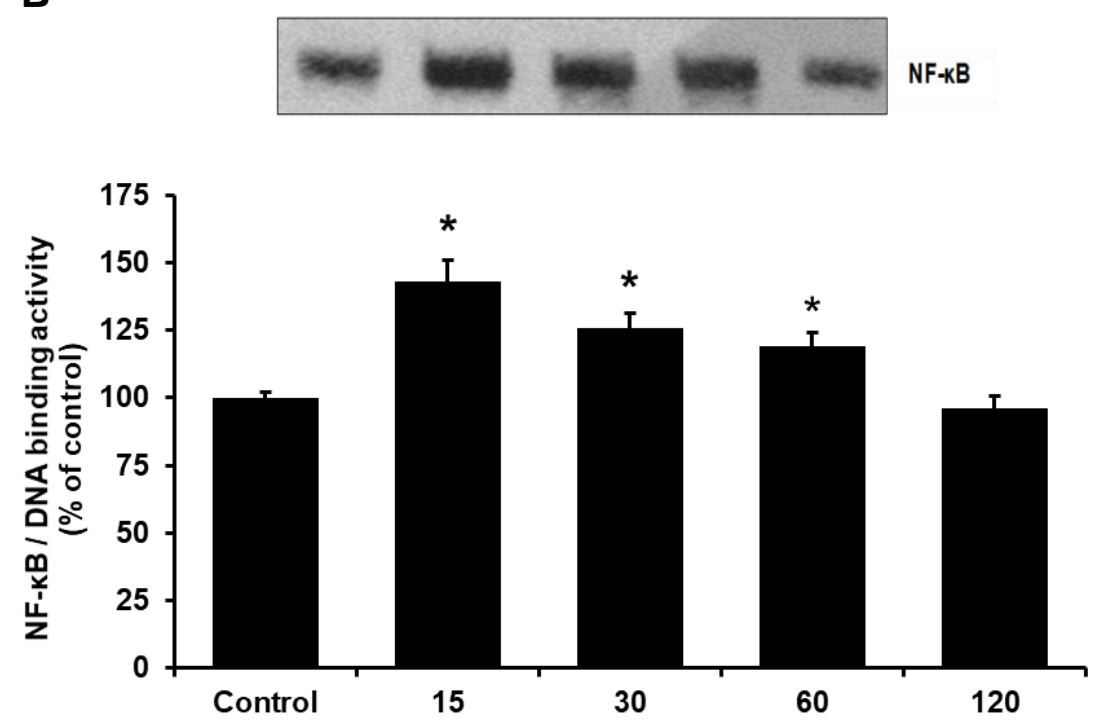

C
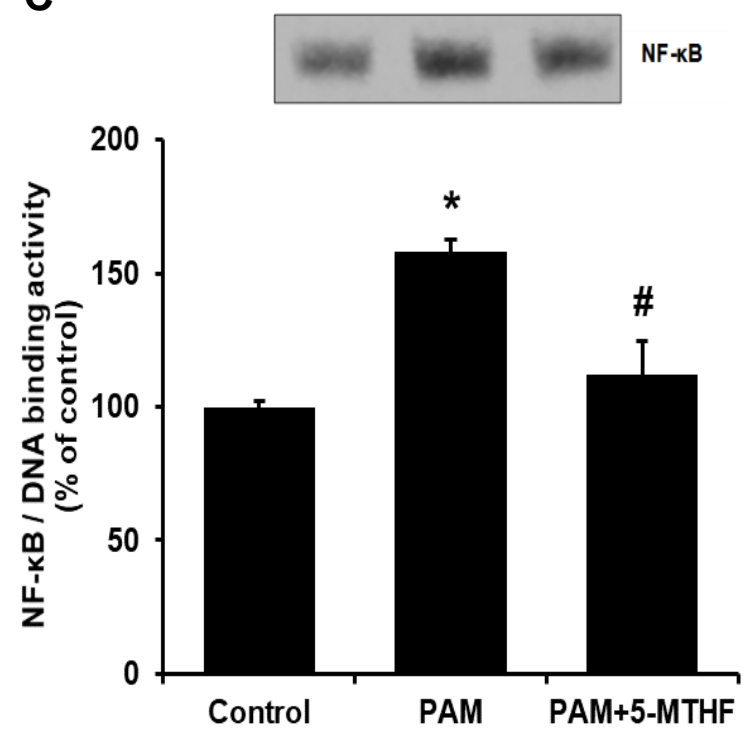

Figure 3.22. Effect of 5-MTHF on inflammatory cytokine expression and NF-кB binding activity in HepG2 cells

HepG2 cells were pretreated overnight with or without 5-methyltetrahydrofolate (5-MTHF, $1 \mu \mathrm{g} / \mathrm{ml})$. Cells were subsequently incubated in the absence (control) or presence of PAM ( 0.3 $\mathrm{mM}$ ). (A) IL-6 mRNA expression was determined in hepatocytes treated with PAM for 8 hours. (B) NF- $\mathrm{kB}$ binding activity was determined in cells incubated with PAM for 15, 30, 60 and 120 minutes. (C) NF- $\mathrm{KB}$ binding activity was determined in cells pretreated with or without 5 -MTHF or PAM. The results are expressed as the means \pm S.E.M. $(\mathrm{n}=3-4) .{ }^{*} p<0.05$ when compared with the value obtained from the control group. \#, $p<0.05$ when compared with the value obtained from PAM group. 


\section{A}
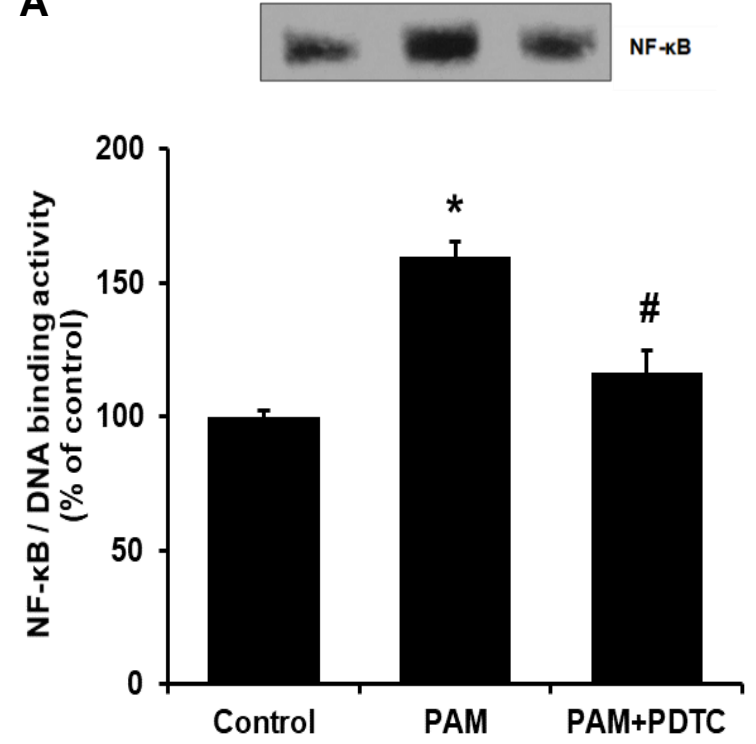

B

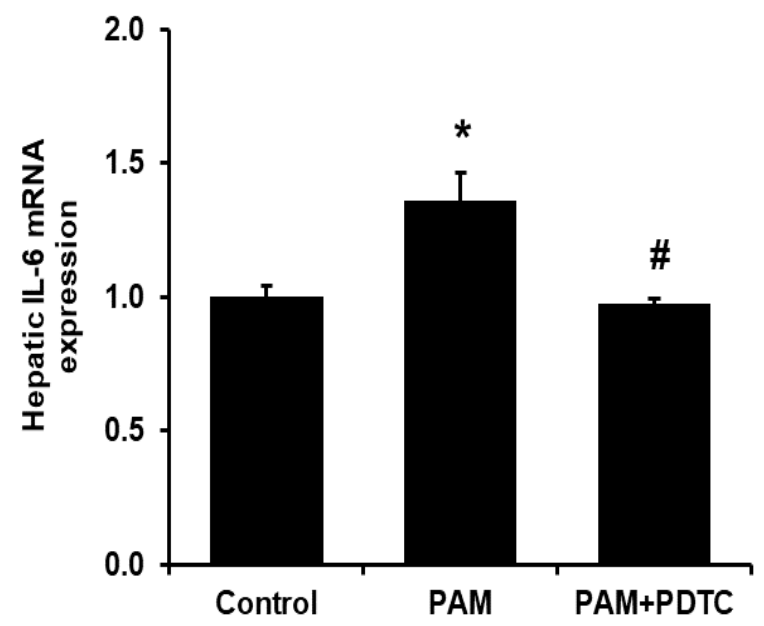

Figure 3.23. Inhibition of NF- $\kappa B$ activation in HepG2 cells

Cells were incubated in the absence (control) or presence of PAM $(0.3 \mathrm{mM})$. In one set of cells,

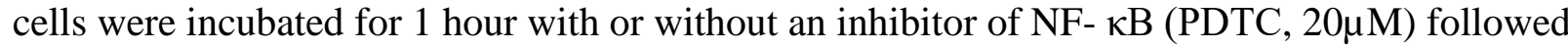
by incubating with PAM $(0.3 \mathrm{mM})$. (A) NF- $\mathrm{KB}$ binding activity was determined in cells after incubation with PAM for 15 minutes. (B) IL-6 mRNA expression was determined in cells after incubation with PAM for 8 hours. The results are expressed as the means \pm S.E.M. $(\mathrm{n}=4) .{ }^{*} p$ $<0.05$ when compared with the value obtained from the control group. \#, $p<0.05$ when compared with the value obtained from PAM group. 


\subsubsection{Discussion}

Chronic activation of inflammatory response is one of the important mechanisms of NAFLD pathogenesis (Kleiner et al. 2005, Cohen et al. 2011). Results from the present study clearly demonstrated that mice fed a high-fat diet for 8 weeks developed features of fatty liver, including hepatic lipid accumulation and increased expression of pro-inflammatory cytokines (IL-6, TNF$\alpha$ ). Folic acid supplementation had a protective effect against high-fat diet-induced hepatic inflammation through inhibition of $\mathrm{NF}-\kappa \mathrm{B}$ activation and subsequently, reduction of inflammatory cytokine expression. The IL-6 and TNF- $\alpha$ cytokines are among the major inflammatory mediators that participate in the development and progression of the chronic inflammatory state associated with NAFLD (Day 2006, Park et al. 2010). Transcription factor $\mathrm{NF}-\kappa \mathrm{B}$ is one of the key regulators for hepatic genes that encode inflammatory cytokines such as IL-6 and TNF- $\alpha$ (Cai et al. 2005). In the present study, high-fat diet feeding resulted in a significant increase in hepatic cytokine expression and NF- $\kappa \mathrm{B}$ activation. Folic acid supplementation effectively attenuated hepatic activation of $\mathrm{NF}-\kappa \mathrm{B}$ and reduced gene expression of IL-6 and TNF- $\alpha$ in mice fed a high-fat diet. Since I $\kappa$ B is a key inhibitor of NF- $\kappa$ B, it is possible that folic acid supplementation may attenuate NF- $\mathrm{B}$ activation by inhibiting phosphorylation of $\mathrm{I} \kappa \mathrm{B}$ protein and therefore, preventing nuclear translocation of $\mathrm{NF}-\kappa \mathrm{B}$ (Hayden et al. 2008). Alternatively, folic acid may inhibit NF- $\kappa \mathrm{B}$ activation via its antioxidant action as folic acid can directly scavenge ROS (Joshi et al. 2001, Gliszczynska-Swiglo et al. 2007) as well as reduce ROS generation in the liver (Sarna et al. 2012).

It has been reported that inhibition of $\mathrm{NF}-\kappa \mathrm{B}$-induced expression of inflammatory cytokines effectively attenuates the hepatic inflammatory response and the progression of NASH in mice (Beraza et al. 2008). Hepatocytes are liver parenchymal cells that contribute significantly to 
inflammatory cytokine production during the onset of an inflammatory response (Day 2006). To further investigate whether the anti-inflammatory effect of folic acid was mediated via inhibition of NF- $\mathrm{KB}$ activity, experiments were carried out in cultured hepatocytes. Incubation of hepatocytes with palmitic acid significantly increased gene expression of IL-6. Incubation of cells with 5-MTHF (the active form of folate) or PDTC (a NF-kB inhibitor) was able to inhibit the elevation of inflammatory cytokine gene expression induced by palmitic acid. These results suggested that the anti-inflammatory effect of folic acid might be mediated through regulation of NF- $\kappa B$ activity.

Hepatic inflammation is often associated with oxidative stress and hepatocellular injury in NAFLD (Chalasani et al. 2004). Pro-inflammatory mediators such as IL-6 and TNF- $\alpha$ are abundantly expressed in the liver during activation of inflammatory responses (Cai et al. 2005). There is evidence that supports a notion that these inflammatory cytokines play an important role in the progression of fatty liver (steatosis) to NASH (Carter-Kent et al. 2008). An increased influx of free fatty acids to the liver induces lipotoxic injury due to accumulation of toxic lipid intermediates and activation of inflammatory response. Free fatty acid accumulation in the liver may contribute to a chronic inflammatory state observed in NAFLD patients. High concentrations of hepatic free fatty acids can increase fatty acid oxidation, which, in turn, leads to excessive accumulation of reactive oxygen species (ROS) and triggers NF-kB-mediated inflammatory signaling in hepatocytes (Joshi-Barve et al. 2007, Neuschwander-Tetri 2010). It has been reported that patients with NAFLD have increased hepatic expression of IL-6 and TNF$\alpha$, which positively correlates with a high degree of inflammatory activity assessed by liver biopsy (Crespo et al. 2001, Wieckowska et al. 2008). Increased hepatic inflammation is also associated with elevated levels of inflammatory cytokines in the circulation (Cai et al. 2005). 
Pro-inflammatory cytokines (IL-6, TNF- $\alpha$ ) can disrupt hepatic insulin signaling and impair glucose metabolism in the liver (Carter-Kent et al. 2008). It has been suggested that activation of NF-אB-mediated inflammatory cytokine production in hepatocytes can induce hepatic insulin resistance (Cai et al. 2005). Our recent study has shown that high-fat diet feeding results in an elevation of serum glucose and insulin levels in mice (Sid et al. 2015). Folic acid supplementation can restore serum glucose and insulin levels in high-fat diet fed mice (Sid et al. 2015). Taken together, our results suggest that attenuation of inflammatory response by folic acid might improve hepatic metabolism during high-fat diet feeding. However, cautions should be made when findings are translated from rodents to humans because rodents appear to metabolize folic acid more efficiently and may be able to tolerate higher doses of folic acid as compared to humans (Bailey et al. 2009). Proper human clinical trials should be conducted in future studies to ascertain the optimal dose for the use of folic acid as a therapy for metabolic disorders.

In conclusion, our study, for the first time, revealed that folic acid supplementation reduced hepatic inflammation in mice fed a high-fat diet. Such an anti-inflammatory effect was mediated through inhibition of NF- $\mathrm{kB}$-induced pro-inflammatory cytokine expression in the liver. Currently, there are limited therapeutic strategies available for patients with NAFLD. As the prevalence of NAFLD continues to increase worldwide, alternative options for NAFLD management is urgently required. The ability of folic acid to suppress the hepatic inflammatory response suggests that this micronutrient may have a therapeutic potential for the management of NAFLD. 


\section{GENERAL DISCUSSION}




\subsection{General discussion}

It is evident that chronic consumption of high-fat diets can impair lipid and glucose metabolism, as well as promote activation of the inflammatory response in the liver of mice. The novel findings of the present study are i) high-fat diet feeding reduces endogenous folate levels by attenuating the expression of hepatic folate transporters in mice; ii) folic acid supplementation effectively improves hepatic lipid and glucose metabolism by restoring AMPK activation in high-fat diet fed mice; iii) folic acid supplementation significantly reduces hepatic inflammation through inhibiting NF- $\kappa \mathrm{B}$ activation in high-fat diet fed mice.

\section{i. Study 1- High-fat diet consumption reduces hepatic folate transporter expression via nuclear respiratory factor-1}

Obesity is a major risk factor of NAFLD. A previous study reported that low serum folate concentrations in obese patients directly correlated with increased severity of steatosis after adjusting for gender, age and BMI (Xia et al. 2018). This suggests that low circulating folate levels might be an independent risk factor for NAFLD. Patients with obesity and NAFLD often exhibit higher energy intake, particularly from dietary fats, compared to healthy individuals (Capristo et al. 2005, Vilar et al. 2008). In the first study, we investigated the effect of high-fat diets on folate status in mice. Our results demonstrated that high-fat diet feeding in mice caused increased hepatic lipid accumulation, which was accompanied by decreased folate levels in the serum and liver. Low folate status was associated with a marked reduction in the mRNA and protein expression of major hepatic folate transporters (PCFT, RFC) in high-fat diet fed mice.

The NRF-1 is an important transcriptional factor that has been shown to regulate gene expression of folate transporters such as PCFT and RFC in the intestine (Gonen et al. 2010). In the present 
study, hepatic NRF-1 binding activity was attenuated following high-fat diet feeding in mice. Moreover, knockdown of NRF-1 gene significantly inhibited the expression of PCFT and RFC, as well as reduced folate levels in hepatocytes. These results indicate that NRF-1 is responsible for regulation of hepatic folate transporters and folate content in the body.

For the first time, our study showed that chronic consumption of dietary fats impairs expression of hepatic PCFT and RFC through inhibition of NRF-1, leading to reduced folate levels in the liver and circulation. Our findings suggest that regulation of folate transporter expression in the liver is an important mechanism to maintain folate homeostasis in the body. Folate serves as a coenzyme for one-carbon transfer reactions involved in nucleic acid biosynthesis, mitochondrial and chloroplast protein synthesis, methylation, as well as amino acid and vitamin metabolism (Tibbetts et al. 2010). The bioactive form of folate, 5-MTHF, is essential for remethylation of homocysteine to methionine, which in turn, leads to the synthesis of SAM. It is evident that SAM is an important methyl donor that plays a key role in lipid metabolism (da Silva et al. 2014). In a previous study, it was shown that feeding mice a folate deficient diet induced hepatic steatosis (Christensen et al. 2010). Folate deficiency compromises methylation capacity and promotes hepatic lipid accumulation by impairing VLDL synthesis and increasing triglyceride synthesis (Vance et al. 2007, Jacobs et al. 2008), as well as attenuating carnitine synthesis. Inhibition of carnitine synthesis may attenuate fatty acid transport to the mitochondria for $\beta$ oxidation, which in turn, promotes lipid accumulation (Malaguarnera et al. 2010, Magoulas et al. 2012). Moreover, folate deficiency promotes increased expression of lipid biosynthetic genes which may also contribute to the development of steatosis (Champier et al. 2012). This suggests that folate depletion caused by high-fat diets can impair hepatic folate storage and might be one of the underlying causes of fatty liver. 


\section{ii. Study 2- Folic acid supplementation during high-fat diet feeding restores AMPK activation via an AMP-LKB1-dependent mechanism}

In a recent study, we have demonstrated that folic acid supplementation can significantly reduce hepatic lipid accumulation in mice fed a high-fat diet (Sarna et al. 2012). The second study investigated the mechanisms by which folic acid regulated lipid and glucose metabolism in the liver. Consumption of high-fat diets increased body weight gain, as well as significantly elevated glucose and lipid levels in the liver. The novel findings from our second study was that folic acid supplementation during high-fat diet feeding lowered hepatic cholesterol and glucose levels by restoration of AMPK phosphorylation (activation). Activation of AMPK by folic acid was mediated through an elevation of its allosteric activator AMP and phosphorylation of its upstream kinase, namely, liver kinase B1 (LKB1) in the liver. Although folic acid supplementation did not affect the body weight gain in high-fat diet fed mice, it was able to significantly improve cholesterol and glucose levels in these animals. This suggests that folic acid exerts its beneficial effects independently from modulation of body weight. Similarly, incubation of hepatocytes with palmitic acid significantly decreased phosphorylation (activation) of AMPK and LKB1. Treatment with 5-MTHF (bioactive form of folate) was able to reverse the inhibitory effect of palmitic acid on phosphorylation of both kinases. Taken together, the results from this study demonstrate that folic acid supplementation mediates AMPK activation, which contributes to improvements in glucose and lipid metabolism that is impaired by high-fat diet feeding in mice.

Our study is among the first to identify the mechanism by which folic acid regulates hepatic AMPK activation in the high-fat diet mouse model. AMPK regulates the activity of enzymes and transcriptional factors involved in lipid and glucose metabolism via phosphorylation (Canto et al. 
2010). AMPK-mediated phosphorylation of various enzymatic substrates plays a crucial role in regulation of metabolic homeostasis in the liver (Hardie et al. 2012). Abnormal lipid and carbohydrate metabolism is often associated with dysregulation of hepatic AMPK (Viollet et al. 2006). It is evident that energy homeostasis is perturbed and hepatic AMPK activity is downregulated during high-fat diet feeding (Rui 2014). Inactivation of AMPK promotes de novo lipogenesis and attenuates fatty acid oxidation in the liver, which may contribute to the development of fatty liver. In addition, AMPK action is implicated in the regulation of glucose homeostasis (Viollet et al. 2006, Canto et al. 2010). The ability of AMPK to effectively suppress gluconeogenesis is impaired during high-fat diet feeding. As a result, this leads to perturbed glucose metabolism in the liver that in turn, promotes development of hyperglycemia (Sid et al. 2015). Activation of AMPK appears to be responsible for metabolic improvements in the liver and plays an essential role in mediating beneficial effects of many pharmaceuticals/ nutraceuticals (Smith et al. 2016). Results from this study suggest that AMPK activation by folic acid is beneficial for management of high-fat diet-induced metabolic disorders such as NAFLD. Dysregulation of folate-dependent one-carbon metabolism due to insufficient folate levels has been implicated in NAFLD-related comorbidities such as obesity, type 2 diabetes, and metabolic syndrome (Hirsch et al. 2005, Mahabir et al. 2008, da Silva et al. 2014, Nilsson et al. 2015). Therefore, folic acid supplementation might be important to restore metabolic processes that are perturbed during chronic high-fat diet consumption.

\section{iii. Study 3- Folic acid supplementation attenuates chronic hepatic inflammation in high-fat diet fed mice}

Hepatic inflammation is an important mediator of NAFLD pathogenesis. It was previously demonstrated that prolonged consumption of high-fat diets leads to significant increases in serum 
fatty acid concentrations in rodents (Cai et al. 2005). Exposure to high concentrations of fatty acids is hepatotoxic and can trigger activation of the inflammatory response in the liver (JoshiBarve et al. 2007). The third study investigated the effect of folic acid supplementation on hepatic inflammation and the mechanisms involved. The results in this study showed that mice fed a high-fat displayed increased deposition of inflammatory foci in the liver, which was associated with elevated expression of hepatic inflammatory cytokines such as IL- 6 and TNF- $\alpha$. For the first time, our study demonstrated that folic acid supplementation during high-fat diet feeding effectively attenuated the inflammatory response by reducing the number of inflammatory foci aggregates and the expression of inflammatory cytokines in the liver of mice. Consistent with the in vivo findings, incubation of hepatocytes with 5-MTHF significantly inhibited expression of IL- 6 and TNF- $\alpha$. The anti-inflammatory effect of folic acid was mediated via inhibition of NF-kB, a key transcriptional regulator of pro-inflammatory genes. Our findings suggest that the hepatoprotective effect of folic acid in NAFLD may be attributed, in part, to its anti-inflammatory action.

An increased supply of free fatty acids to the liver has been shown to induce lipotoxicity, which plays a major role in the development of hepatic inflammation in NAFLD (Joshi-Barve et al. 2007). Asides from direct hepatic lipotoxicity induced by excessive accumulation of free fatty acids, other mechanisms may be involved in triggering the activation of inflammatory response in the liver. Oxidative stress can cause hepatocellular injury by stimulating activation of NF-kB, which mediates expression of inflammatory cytokines by liver parenchymal and immune cells. A previous study conducted in our lab demonstrated that folic acid supplementation can effectively attenuate hepatic oxidative stress induced by high-fat diet feeding in mice (Sarna et al. 2012). The ability of folic acid to improve oxidative stress may contribute to its anti-inflammatory 
effects (Seki et al. 2002, Rolo et al. 2012). Taken together, chronic hepatic inflammation can drive the progression of steatosis to severe stages such as NASH and cirrhosis. Inhibition of the inflammatory response may be a promising strategy for preventing NAFLD pathogenesis. Since folic acid is shown to effectively attenuate hepatic inflammation, this suggests that this vitamin might have an important role in the management of NAFLD.

\subsection{Conclusions}

Our study in the high-fat diet mouse model has identified the involvement of folate in the development of NAFLD. NAFLD is a multifaceted disorder with many underlying metabolic abnormalities attributed to impaired regulation of lipid and glucose metabolism, as well as dysregulation of the inflammatory response. Reduced folate levels caused by chronic consumption of high-fat diets may contribute to the development of metabolic abnormalities associated with NAFLD. The ability of folic acid to restore metabolic and inflammatory processes that are perturbed by high-fat diets suggests that this vitamin may potentially have therapeutic implications for the management of NAFLD and its associated comorbidities such as obesity and type 2 diabetes. Our findings suggest that patients with NAFLD and obesity may be at a higher risk for folate deficiency regardless of achieving proper folate intakes. Understanding the mechanisms by which folate regulates metabolic processes may position this vitamin as a potential therapeutic for NAFLD. 


\section{FUTURE PERSPECTIVES}


One of the novel findings in this study is that chronic consumption of high-fat diets impairs regulation of folate homeostasis, which is attributed to downregulation of the major hepatic folate transporters, PCFT and RFC. It is evident that the liver is the major organ responsible for folate storage and metabolism (Wright et al. 2007). Although PCFT and RFC are well characterized in the intestine, their role in the liver is not well studied. Our study demonstrates that downregulation of PCFT and RFC in the liver leads to a significant reduction of folate levels in the liver and circulation of high-fat diet fed mice. Another novel finding in our study is that NRF-1 is one of the transcriptional factors that is responsible for modulating gene expression of hepatic PCFT and RFC, which in turn, regulates folate levels in the liver and serum. However, the mechanism that is responsible for regulation of NRF-1 remains to be investigated. Serine phosphorylation of NRF-1 was demonstrated to enhance the DNA binding activity of this transcriptional factor (Gugneja et al. 1997). It may be useful to identify other post-translational modifications that may affect NRF-1 mediated regulation of folate transporters and folate levels in the liver. Moreover, it was previously reported that patients with obesity and NAFLD exhibited low levels of folate in the circulation. Although our present study demonstrates that high-fat diets can significantly lower endogenous folate status, it is unclear whether depletion of folates is a cause or a consequence of NAFLD. Future studies are necessary to determine whether low folate status may be a risk factor for NAFLD.

The novel findings presented in this study were that folic acid supplementation enhances AMPK activity in the liver via restoration of AMP levels and activation of its upstream kinase, LKB1. Our study suggests that activation of AMPK leads to a decrease in hepatic lipid levels, as well as glucose levels in the serum of high-fat diet fed mice. However, it is possible that regulation of other metabolic enzymes may also play a role. Further studies are necessary to identify other 
potential mechanisms by which folic acid supplementation may mediate its effects on hepatic lipid and glucose metabolism. As folic acid seems to play a significant role in reducing serum glucose levels that were elevated during high-fat diet feeding in mice, it would be intriguing to investigate whether folic acid supplementation may affect hepatic insulin sensitivity or glucose tolerance in these mice. Although folic acid supplementation appears to improve the metabolic profile of high-fat diet fed mice, there are concerns regarding the dose of folic acid supplementation. High doses of folic acid may cause negative effects such as masking vitamin B12 deficiency and impairing cognitive function (Lucock 2000). As mice can efficiently metabolize and tolerate higher doses of folic acid compared to humans (Bailey et al. 2009), caution should be taken when findings are translated from rodents to humans. Proper clinical trials are warranted to ascertain the optimal dose of folic acid supplementation in specific populations such as individuals with fatty liver or metabolic disease to minimize adverse effects.

The novel finding in our third study is that folic acid exhibits anti-inflammatory effects due to its ability to effectively suppress NF- $\mathrm{kB}$ mediated gene expression of hepatic inflammatory cytokines (IL-6, TNF- $\alpha$ ) in high-fat diet fed mice. The ability of folic acid to reduce hepatic inflammation caused by high-fat diet feeding suggests that folic acid supplementation may be beneficial to prevent pathogenesis of steatosis to severe stages of NAFLD such as NASH and cirrhosis. Similarly, incubation with 5-MTHF is able to attenuate expression of inflammatory cytokines in palmitic acid-treated hepatocytes. Hepatocytes are the liver parenchymal cells that significantly contribute to the production of inflammatory cytokines. However, immune cells such as kupffer cells (liver resident macrophages) and neutrophils may also generate and secrete inflammatory mediators during the onset of hepatic inflammatory response (Bieghs et al. 2013). It would be of interest to investigate whether folate treatment can also suppress the generation of 
inflammatory cytokines by other liver immune cells. Moreover, hepatocytes express toll-like receptor 4 (TLR4), which is typically activated in high-fat diet fed mice and obese patients with NAFLD (Sharifnia et al. 2015). Activation of TLR4 stimulates the generation of proinflammatory cytokines via upregulation of several inflammatory transcriptional factors including NF-kB, activator protein-1 (AP-1) and interferon regulatory factors (IRFs) (O'Neill et al. 2013). Further studies are needed to determine whether inactivation of NF- $\kappa B$ by folic acid is mediated through inhibition of TLR4. Though our study suggests that folic acid supplementation can inhibit the hepatic inflammatory response in high-fat diet fed mice, the effect of folic acid supplementation on anti-inflammatory cytokine production by liver immune cells remains to be investigated. 


\section{REFERENCES}


Adams, L. A., J. F. Lymp, J. St Sauver, S. O. Sanderson, K. D. Lindor, A. Feldstein and P. Angulo (2005). The natural history of nonalcoholic fatty liver disease: a population-based cohort study. Gastroenterology 129(1): 113-121.

Adiels, M., M. R. Taskinen, C. Packard, M. J. Caslake, A. Soro-Paavonen, J. Westerbacka, S. Vehkavaara, A. Hakkinen, S. O. Olofsson, H. Yki-Jarvinen and J. Boren (2006). Overproduction of large VLDL particles is driven by increased liver fat content in man. Diabetologia 49(4): 755765.

Afdhal, N. H. (2012). Fibroscan (transient elastography) for the measurement of liver fibrosis. Gastroenterol Hepatol (N Y) 8(9): 605-607.

Ahmad Najar, R., B. Rahat, A. Hussain, S. Thakur, J. Kaur, J. Kaur and A. Hamid (2016). Gene specific epigenetic regulation of hepatic folate transport system is responsible for perturbed cellular folate status during aging and exogenous modulation. Mol Nutr Food Res 60(6): 15011513.

Ahmed, A., R. J. Wong and S. A. Harrison (2015). Nonalcoholic Fatty Liver Disease Review: Diagnosis, Treatment, and Outcomes. Clin Gastroenterol Hepatol 13(12): 2062-2070.

Ahmed, M. (2015). Non-alcoholic fatty liver disease in 2015. World J Hepatol 7(11): 1450-1459.

Akkaoui, M., I. Cohen, C. Esnous, V. Lenoir, M. Sournac, J. Girard and C. Prip-Buus (2009). Modulation of the hepatic malonyl-CoA-carnitine palmitoyltransferase 1A partnership creates a metabolic switch allowing oxidation of de novo fatty acids. Biochem J 420(3): 429-438.

Alkhouri, N., L. J. Dixon and A. E. Feldstein (2009). Lipotoxicity in nonalcoholic fatty liver disease: not all lipids are created equal. Expert Rev Gastroenterol Hepatol 3(4): 445-451.

Amarapurkar, D., P. Kamani, N. Patel, P. Gupte, P. Kumar, S. Agal, R. Baijal, S. Lala, D. Chaudhary and A. Deshpande (2007). Prevalence of non-alcoholic fatty liver disease: population based study. Ann Hepatol 6(3): 161-163.

Andreelli, F., M. Foretz, C. Knauf, P. D. Cani, C. Perrin, M. A. Iglesias, B. Pillot, A. Bado, F. Tronche, G. Mithieux, S. Vaulont, R. Burcelin and B. Viollet (2006). Liver adenosine monophosphate-activated kinase-alpha2 catalytic subunit is a key target for the control of hepatic glucose production by adiponectin and leptin but not insulin. Endocrinology 147(5): 2432-2441.

Angulo, P. (2002). Nonalcoholic fatty liver disease. N Engl J Med 346(16): 1221-1231. 
Angulo, P. (2007). Obesity and nonalcoholic fatty liver disease. Nutr Rev 65(6 Pt 2): S57-63.

Angulo, P., J. M. Hui, G. Marchesini, E. Bugianesi, J. George, G. C. Farrell, F. Enders, S. Saksena, A. D. Burt, J. P. Bida, K. Lindor, S. O. Sanderson, M. Lenzi, L. A. Adams, J. Kench, T. M. Therneau and C. P. Day (2007). The NAFLD fibrosis score: a noninvasive system that identifies liver fibrosis in patients with NAFLD. Hepatology 45(4): 846-854.

Angulo, P., D. E. Kleiner, S. Dam-Larsen, L. A. Adams, E. S. Bjornsson, P. Charatcharoenwitthaya, P. R. Mills, J. C. Keach, H. D. Lafferty, A. Stahler, S. Haflidadottir and F. Bendtsen (2015). Liver Fibrosis, but No Other Histologic Features, Is Associated With Longterm Outcomes of Patients With Nonalcoholic Fatty Liver Disease. Gastroenterology 149(2): 389-397.e310.

Anstee, Q. M. and R. D. Goldin (2006). Mouse models in non-alcoholic fatty liver disease and steatohepatitis research. Int J Exp Pathol 87(1): 1-16.

Anstee, Q. M., G. Targher and C. P. Day (2013). Progression of NAFLD to diabetes mellitus, cardiovascular disease or cirrhosis. Nat Rev Gastroenterol Hepatol 10(6): 330-344.

Argo, C. K. and S. H. Caldwell (2009). Epidemiology and natural history of non-alcoholic steatohepatitis. Clin Liver Dis 13(4): 511-531.

Arguello, G., E. Balboa, M. Arrese and S. Zanlungo (2015). Recent insights on the role of cholesterol in non-alcoholic fatty liver disease. Biochim Biophys Acta 1852(9): 1765-1778.

Arkan, M. C., A. L. Hevener, F. R. Greten, S. Maeda, Z. W. Li, J. M. Long, A. Wynshaw-Boris, G. Poli, J. Olefsky and M. Karin (2005). IKK-beta links inflammation to obesity-induced insulin resistance. Nat Med 11(2): 191-198.

Asgharpour, A., S. C. Cazanave, T. Pacana, M. Seneshaw, R. Vincent, B. A. Banini, D. P. Kumar, K. Daita, H. K. Min, F. Mirshahi, P. Bedossa, X. Sun, Y. Hoshida, S. V. Koduru, D. Contaifer, Jr., U. O. Warncke, D. S. Wijesinghe and A. J. Sanyal (2016). A diet-induced animal model of non-alcoholic fatty liver disease and hepatocellular cancer. J Hepatol 65(3): 579-588.

Assifi, M. M., G. Suchankova, S. Constant, M. Prentki, A. K. Saha and N. B. Ruderman (2005). AMP-activated protein kinase and coordination of hepatic fatty acid metabolism of starved/carbohydrate-refed rats. Am J Physiol Endocrinol Metab 289(5): E794-800. 
Athyros, V. G., K. Tziomalos, T. D. Gossios, T. Griva, P. Anagnostis, K. Kargiotis, E. D. Pagourelias, E. Theocharidou, A. Karagiannis and D. P. Mikhailidis (2010). Safety and efficacy of long-term statin treatment for cardiovascular events in patients with coronary heart disease and abnormal liver tests in the Greek Atorvastatin and Coronary Heart Disease Evaluation (GREACE) Study: a post-hoc analysis. Lancet 376(9756): 1916-1922.

Au-Yeung, K. K., J. C. Yip, Y. L. Siow and K. O (2006). Folic acid inhibits homocysteineinduced superoxide anion production and nuclear factor kappa B activation in macrophages. Can J Physiol Pharmacol 84(1): 141-147.

Avalos, J. L., K. M. Bever and C. Wolberger (2005). Mechanism of sirtuin inhibition by nicotinamide: altering the $\mathrm{NAD}(+)$ cosubstrate specificity of a Sir2 enzyme. Mol Cell 17(6): 855868.

Bailey, R. L., K. W. Dodd, J. J. Gahche, J. T. Dwyer, M. A. McDowell, E. A. Yetley, C. A. Sempos, V. L. Burt, K. L. Radimer and M. F. Picciano (2010). Total folate and folic acid intake from foods and dietary supplements in the United States: 2003-2006. Am J Clin Nutr 91(1): 231237.

Bailey, S. W. and J. E. Ayling (2009). The extremely slow and variable activity of dihydrofolate reductase in human liver and its implications for high folic acid intake. Proc Natl Acad Sci U S A 106(36): 15424-15429.

Baker, R. G., M. S. Hayden and S. Ghosh (2011). NF-kappaB, inflammation, and metabolic disease. Cell Metab 13(1): 11-22.

Barlowe, C. K. and D. R. Appling (1988). In vitro evidence for the involvement of mitochondrial folate metabolism in the supply of cytoplasmic one-carbon units. Biofactors 1(2): 171-176.

Barnes, P. J. and M. Karin (1997). Nuclear factor-kappaB: a pivotal transcription factor in chronic inflammatory diseases. N Engl J Med 336(15): 1066-1071.

Bechmann, L. P., R. A. Hannivoort, G. Gerken, G. S. Hotamisligil, M. Trauner and A. Canbay (2012). The interaction of hepatic lipid and glucose metabolism in liver diseases. J Hepatol 56(4): 952-964.

Belfort, R., S. A. Harrison, K. Brown, C. Darland, J. Finch, J. Hardies, B. Balas, A. Gastaldelli, F. Tio, J. Pulcini, R. Berria, J. Z. Ma, S. Dwivedi, R. Havranek, C. Fincke, R. DeFronzo, G. A. Bannayan, S. Schenker and K. Cusi (2006). A placebo-controlled trial of pioglitazone in subjects with nonalcoholic steatohepatitis. N Engl J Med 355(22): 2297-2307. 
Beraza, N., Y. Malato, S. Vander Borght, C. Liedtke, H. E. Wasmuth, M. Dreano, R. de Vos, T. Roskams and C. Trautwein (2008). Pharmacological IKK2 inhibition blocks liver steatosis and initiation of non-alcoholic steatohepatitis. Gut 57(5): 655-663.

Bergeron, R., S. F. Previs, G. W. Cline, P. Perret, R. R. Russell, 3rd, L. H. Young and G. I. Shulman (2001). Effect of 5-aminoimidazole-4-carboxamide-1-beta-D-ribofuranoside infusion on in vivo glucose and lipid metabolism in lean and obese Zucker rats. Diabetes 50(5): 10761082.

Bhargava, R., P. A. Senior, T. E. Ackerman, E. A. Ryan, B. W. Paty, J. R. Lakey and A. M. Shapiro (2004). Prevalence of hepatic steatosis after islet transplantation and its relation to graft function. Diabetes 53(5): 1311-1317.

Bieghs, V. and C. Trautwein (2013). The innate immune response during liver inflammation and metabolic disease. Trends Immunol 34(9): 446-452.

Bird, J. K., A. G. Ronnenberg, S. W. Choi, F. Du, J. B. Mason and Z. Liu (2015). Obesity is associated with increased red blood cell folate despite lower dietary intakes and serum concentrations. J Nutr 145(1): 79-86.

Birkenfeld, A. L. and G. I. Shulman (2014). Nonalcoholic fatty liver disease, hepatic insulin resistance, and type 2 diabetes. Hepatology 59(2): 713-723.

Blom, H. J., G. M. Shaw, M. den Heijer and R. H. Finnell (2006). Neural tube defects and folate: case far from closed. Nat Rev Neurosci 7(9): 724-731.

Boushey, C. J., S. A. Beresford, G. S. Omenn and A. G. Motulsky (1995). A quantitative assessment of plasma homocysteine as a risk factor for vascular disease. Probable benefits of increasing folic acid intakes. JAMA 274(13): 1049-1057.

Brattstrom, L., D. E. Wilcken, J. Ohrvik and L. Brudin (1998). Common methylenetetrahydrofolate reductase gene mutation leads to hyperhomocysteinemia but not to vascular disease: the result of a meta-analysis. Circulation 98(23): 2520-2526.

Bray, G. A. and D. A. York (1979). Hypothalamic and genetic obesity in experimental animals: an autonomic and endocrine hypothesis. Physiol Rev 59(3): 719-809. 
Brix, A. E., A. Elgavish, T. R. Nagy, B. A. Gower, W. J. Rhead and P. A. Wood (2002). Evaluation of liver fatty acid oxidation in the leptin-deficient obese mouse. Mol Genet Metab 75(3): 219-226.

Brown, M. S. and J. L. Goldstein (2008). Selective versus total insulin resistance: a pathogenic paradox. Cell Metab 7(2): 95-96.

Brown, M. S., P. T. Kovanen and J. L. Goldstein (1981). Regulation of plasma cholesterol by lipoprotein receptors. Science 212(4495): 628-635.

Browning, J. D., L. S. Szczepaniak, R. Dobbins, P. Nuremberg, J. D. Horton, J. C. Cohen, S. M. Grundy and H. H. Hobbs (2004). Prevalence of hepatic steatosis in an urban population in the United States: impact of ethnicity. Hepatology 40(6): 1387-1395.

Brunt, E. M. and D. G. Tiniakos (2010). Histopathology of nonalcoholic fatty liver disease. World J Gastroenterol 16(42): 5286-5296.

Bryant, N. J., R. Govers and D. E. James (2002). Regulated transport of the glucose transporter GLUT4. Nat Rev Mol Cell Biol 3(4): 267-277.

Buettner, R., J. Scholmerich and L. C. Bollheimer (2007). High-fat diets: modeling the metabolic disorders of human obesity in rodents. Obesity (Silver Spring) 15(4): 798-808.

Bugianesi, E., E. Gentilcore, R. Manini, S. Natale, E. Vanni, N. Villanova, E. David, M. Rizzetto and G. Marchesini (2005). A randomized controlled trial of metformin versus vitamin E or prescriptive diet in nonalcoholic fatty liver disease. Am J Gastroenterol 100(5): 1082-1090.

Buhl, E. S., N. Jessen, O. Schmitz, S. B. Pedersen, O. Pedersen, G. D. Holman and S. Lund (2001). Chronic treatment with 5-aminoimidazole-4-carboxamide-1-beta-D-ribofuranoside increases insulin-stimulated glucose uptake and GLUT4 translocation in rat skeletal muscles in a fiber type-specific manner. Diabetes 50(1): 12-17.

Burda, P., A. Schafer, T. Suormala, T. Rummel, C. Burer, D. Heuberger, M. Frapolli, C. Giunta, J. Sokolova, H. Vlaskova, V. Kozich, H. G. Koch, B. Fowler, D. S. Froese and M. R. Baumgartner (2015). Insights into severe 5,10-methylenetetrahydrofolate reductase deficiency: molecular genetic and enzymatic characterization of 76 patients. Hum Mutat 36(6): 611-621. 
Cai, D., M. Yuan, D. F. Frantz, P. A. Melendez, L. Hansen, J. Lee and S. E. Shoelson (2005). Local and systemic insulin resistance resulting from hepatic activation of IKK-beta and NFkappaB. Nat Med 11(2): 183-190.

Camilo, E., J. Zimmerman, J. B. Mason, B. Golner, R. Russell, J. Selhub and I. H. Rosenberg (1996). Folate synthesized by bacteria in the human upper small intestine is assimilated by the host. Gastroenterology 110(4): 991-998.

Canto, C. and J. Auwerx (2010). AMP-activated protein kinase and its downstream transcriptional pathways. Cell Mol Life Sci 67(20): 3407-3423.

Capristo, E., L. Miele, A. Forgione, V. Vero, S. Farnetti, G. Mingrone, A. V. Greco, G. Gasbarrini and A. Grieco (2005). Nutritional aspects in patients with non-alcoholic steatohepatitis (NASH). Eur Rev Med Pharmacol Sci 9(5): 265-268.

Cario, H., D. E. Smith, H. Blom, N. Blau, H. Bode, K. Holzmann, U. Pannicke, K. P. Hopfner, E. M. Rump, Z. Ayric, E. Kohne, K. M. Debatin, Y. Smulders and K. Schwarz (2011). Dihydrofolate reductase deficiency due to a homozygous DHFR mutation causes megaloblastic anemia and cerebral folate deficiency leading to severe neurologic disease. Am J Hum Genet 88(2): 226-231.

Carter-Kent, C., N. N. Zein and A. E. Feldstein (2008). Cytokines in the pathogenesis of fatty liver and disease progression to steatohepatitis: implications for treatment. Am J Gastroenterol 103(4): 1036-1042.

CDC (2010). CDC Grand Rounds: additional opportunities to prevent neural tube defects with folic acid fortification. MMWR Morb Mortal Wkly Rep 59(31): 980-984.

Chalasani, N., D. W. Crabb, O. W. Cummings, P. Y. Kwo, A. Asghar, P. K. Pandya and R. V. Considine (2003). Does leptin play a role in the pathogenesis of human nonalcoholic steatohepatitis? Am J Gastroenterol 98(12): 2771-2776.

Chalasani, N., M. A. Deeg and D. W. Crabb (2004). Systemic levels of lipid peroxidation and its metabolic and dietary correlates in patients with nonalcoholic steatohepatitis. Am J Gastroenterol 99(8): 1497-1502.

Chalasani, N., Z. Younossi, J. E. Lavine, A. M. Diehl, E. M. Brunt, K. Cusi, M. Charlton and A. J. Sanyal (2012). The diagnosis and management of non-alcoholic fatty liver disease: practice guideline by the American Gastroenterological Association, American Association for the Study 
of Liver Diseases, and American College of Gastroenterology. Gastroenterology 142(7): 15921609.

Champier, J., F. Claustrat, N. Nazaret, M. Fevre Montange and B. Claustrat (2012). Folate depletion changes gene expression of fatty acid metabolism, DNA synthesis, and circadian cycle in male mice. Nutr Res 32(2): 124-132.

Chango, A., A. A. Nour, S. Bousserouel, D. Eveillard, P. M. Anton and J. L. Gueant (2009). Time course gene expression in the one-carbon metabolism network using HepG2 cell line grown in folate-deficient medium. J Nutr Biochem 20(4): 312-320.

Chavez-Tapia, N. C., N. Rosso and C. Tiribelli (2011). In vitro models for the study of nonalcoholic fatty liver disease. Curr Med Chem 18(7): 1079-1084.

Chen, H., O. Charlat, L. A. Tartaglia, E. A. Woolf, X. Weng, S. J. Ellis, N. D. Lakey, J. Culpepper, K. J. Moore, R. E. Breitbart, G. M. Duyk, R. I. Tepper and J. P. Morgenstern (1996). Evidence that the diabetes gene encodes the leptin receptor: identification of a mutation in the leptin receptor gene in $\mathrm{db} / \mathrm{db}$ mice. Cell 84(3): 491-495.

Chen, Z. W., L. Y. Chen, H. L. Dai, J. H. Chen and L. Z. Fang (2008). Relationship between alanine aminotransferase levels and metabolic syndrome in nonalcoholic fatty liver disease. $\underline{\mathbf{J}}$ Zhejiang Univ Sci B 9(8): 616-622.

Chou, Y. F., C. C. Yu and R. F. Huang (2007). Changes in mitochondrial DNA deletion, content, and biogenesis in folate-deficient tissues of young rats depend on mitochondrial folate and oxidative DNA injuries. J Nutr 137(9): 2036-2042.

Christensen, K. E., Q. Wu, X. Wang, L. Deng, M. A. Caudill and R. Rozen (2010). Steatosis in mice is associated with gender, folate intake, and expression of genes of one-carbon metabolism. J Nutr 140(10): 1736-1741.

Christian, F., E. L. Smith and R. J. Carmody (2016). The Regulation of NF-kappaB Subunits by Phosphorylation. Cells 5(1).

Clarke, P. R. and D. G. Hardie (1990). Regulation of HMG-CoA reductase: identification of the site phosphorylated by the AMP-activated protein kinase in vitro and in intact rat liver. EMBO J 9(8): 2439-2446. 
Clarke, R. (2000). Lowering blood homocysteine with folic acid-based supplements: metaanalysis of randomised trials. Indian Heart J 52(7 Suppl): S59-64.

Cohen, J. C., J. D. Horton and H. H. Hobbs (2011). Human fatty liver disease: old questions and new insights. Science 332(6037): 1519-1523.

Cole, B. F., J. A. Baron, R. S. Sandler, R. W. Haile, D. J. Ahnen, R. S. Bresalier, G. McKeownEyssen, R. W. Summers, R. I. Rothstein, C. A. Burke, D. C. Snover, T. R. Church, J. I. Allen, D. J. Robertson, G. J. Beck, J. H. Bond, T. Byers, J. S. Mandel, L. A. Mott, L. H. Pearson, E. L. Barry, J. R. Rees, N. Marcon, F. Saibil, P. M. Ueland and E. R. Greenberg (2007). Folic acid for the prevention of colorectal adenomas: a randomized clinical trial. JAMA 297(21): 2351-2359.

Collins, S., T. L. Martin, R. S. Surwit and J. Robidoux (2004). Genetic vulnerability to dietinduced obesity in the C57BL/6J mouse: physiological and molecular characteristics. Physiol Behav 81(2): 243-248.

Collins, S. P., J. L. Reoma, D. M. Gamm and M. D. Uhler (2000). LKB1, a novel serine/threonine protein kinase and potential tumour suppressor, is phosphorylated by cAMPdependent protein kinase (PKA) and prenylated in vivo. Biochem J 345 Pt 3: 673-680.

Cool, B., B. Zinker, W. Chiou, L. Kifle, N. Cao, M. Perham, R. Dickinson, A. Adler, G. Gagne, R. Iyengar, G. Zhao, K. Marsh, P. Kym, P. Jung, H. S. Camp and E. Frevert (2006). Identification and characterization of a small molecule AMPK activator that treats key components of type 2 diabetes and the metabolic syndrome. Cell Metab 3(6): 403-416.

Coughlan, K. A., R. J. Valentine, N. B. Ruderman and A. K. Saha (2013). Nutrient Excess in AMPK Downregulation and Insulin Resistance. J Endocrinol Diabetes Obes 1(1): 1008.

Crespo, J., A. Cayon, P. Fernandez-Gil, M. Hernandez-Guerra, M. Mayorga, A. DominguezDiez, J. C. Fernandez-Escalante and F. Pons-Romero (2001). Gene expression of tumor necrosis factor alpha and TNF-receptors, p55 and p75, in nonalcoholic steatohepatitis patients. Hepatology 34(6): 1158-1163.

Cui, Z., M. Houweling and D. E. Vance (1994). Suppression of rat hepatoma cell growth by expression of phosphatidylethanolamine N-methyltransferase-2. J Biol Chem 269(40): 2453124533.

da Silva, R. P., K. B. Kelly, A. Al Rajabi and R. L. Jacobs (2014). Novel insights on interactions between folate and lipid metabolism. Biofactors 40(3): 277-283. 
da Silva, V. R., D. B. Hausman, G. P. Kauwell, A. Sokolow, R. L. Tackett, S. L. Rathbun and L. B. Bailey (2013). Obesity affects short-term folate pharmacokinetics in women of childbearing age. Int J Obes (Lond) 37(12): 1608-1610.

Dahlhoff, C., S. Worsch, M. Sailer, B. A. Hummel, J. Fiamoncini, K. Uebel, R. Obeid, C. Scherling, J. Geisel, B. L. Bader and H. Daniel (2014). Methyl-donor supplementation in obese mice prevents the progression of NAFLD, activates AMPK and decreases acyl-carnitine levels. Mol Metab 3(5): 565-580.

Dambach, D. M., B. A. Andrews and F. Moulin (2005). New technologies and screening strategies for hepatotoxicity: use of in vitro models. Toxicol Pathol 33(1): 17-26.

Dashty, M. (2013). A quick look at biochemistry: carbohydrate metabolism. Clin Biochem 46(15): 1339-1352.

Davies, S. P., N. R. Helps, P. T. Cohen and D. G. Hardie (1995). 5'-AMP inhibits dephosphorylation, as well as promoting phosphorylation, of the AMP-activated protein kinase. Studies using bacterially expressed human protein phosphatase-2C alpha and native bovine protein phosphatase-2AC. FEBS Lett 377(3): 421-425.

Day, C. P. (2005). Natural history of NAFLD: remarkably benign in the absence of cirrhosis. Gastroenterology 129(1): 375-378.

Day, C. P. (2006). From fat to inflammation. Gastroenterology 130(1): 207-210.

De Wals, P., F. Tairou, M. I. Van Allen, S. H. Uh, R. B. Lowry, B. Sibbald, J. A. Evans, M. C. Van den Hof, P. Zimmer, M. Crowley, B. Fernandez, N. S. Lee and T. Niyonsenga (2007). Reduction in neural-tube defects after folic acid fortification in Canada. N Engl J Med 357(2): $135-142$.

Despres, J. P. (2003). Potential contribution of metformin to the management of cardiovascular disease risk in patients with abdominal obesity, the metabolic syndrome and type 2 diabetes. Diabetes Metab 29(4 Pt 2): 6S53-61.

Dhonukshe-Rutten, R. A., J. H. de Vries, A. de Bree, N. van der Put, W. A. van Staveren and L. C. de Groot (2009). Dietary intake and status of folate and vitamin B12 and their association with homocysteine and cardiovascular disease in European populations. Eur J Clin Nutr 63(1): 18-30. 
Dietschy, J. M., S. D. Turley and D. K. Spady (1993). Role of liver in the maintenance of cholesterol and low density lipoprotein homeostasis in different animal species, including humans. J Lipid Res 34(10): 1637-1659.

Diop-Bove, N. K., J. Wu, R. Zhao, J. Locker and I. D. Goldman (2009). Hypermethylation of the human proton-coupled folate transporter (SLC46A1) minimal transcriptional regulatory region in an antifolate-resistant HeLa cell line. Mol Cancer Ther 8(8): 2424-2431.

Dixon, J. B., P. S. Bhathal and P. E. O'Brien (2001). Nonalcoholic fatty liver disease: predictors of nonalcoholic steatohepatitis and liver fibrosis in the severely obese. Gastroenterology 121(1): 91-100.

Doege, H., D. Grimm, A. Falcon, B. Tsang, T. A. Storm, H. Xu, A. M. Ortegon, M. Kazantzis, M. A. Kay and A. Stahl (2008). Silencing of hepatic fatty acid transporter protein 5 in vivo reverses diet-induced non-alcoholic fatty liver disease and improves hyperglycemia. J Biol Chem 283(32): 22186-22192.

Donnelly, K. L., C. I. Smith, S. J. Schwarzenberg, J. Jessurun, M. D. Boldt and E. J. Parks (2005). Sources of fatty acids stored in liver and secreted via lipoproteins in patients with nonalcoholic fatty liver disease. J Clin Invest 115(5): 1343-1351.

Eloranta, J. J., Z. M. Zair, C. Hiller, S. Hausler, B. Stieger and G. A. Kullak-Ublick (2009). Vitamin D3 and its nuclear receptor increase the expression and activity of the human protoncoupled folate transporter. Mol Pharmacol 76(5): 1062-1071.

Elsharkawy, A. M. and D. A. Mann (2007). Nuclear factor-kappaB and the hepatic inflammation-fibrosis-cancer axis. Hepatology 46(2): 590-597.

Estes, C., H. Razavi, R. Loomba, Z. Younossi and A. J. Sanyal (2018). Modeling the epidemic of nonalcoholic fatty liver disease demonstrates an exponential increase in burden of disease. Hepatology 67(1): 123-133.

Fabbrini, E., B. S. Mohammed, F. Magkos, K. M. Korenblat, B. W. Patterson and S. Klein (2008). Alterations in adipose tissue and hepatic lipid kinetics in obese men and women with nonalcoholic fatty liver disease. Gastroenterology 134(2): 424-431.

Fabbrini, E., S. Sullivan and S. Klein (2010). Obesity and nonalcoholic fatty liver disease: biochemical, metabolic, and clinical implications. Hepatology 51(2): 679-689. 
Fan, J., J. Ye, J. J. Kamphorst, T. Shlomi, C. B. Thompson and J. D. Rabinowitz (2014). Quantitative flux analysis reveals folate-dependent NADPH production. Nature 510(7504): 298302.

Farrell, G. C. and C. Z. Larter (2006). Nonalcoholic fatty liver disease: from steatosis to cirrhosis. Hepatology 43(2 Suppl 1): S99-S112.

Fast, D. G. and D. E. Vance (1995). Nascent VLDL phospholipid composition is altered when phosphatidylcholine biosynthesis is inhibited: evidence for a novel mechanism that regulates VLDL secretion. Biochim Biophys Acta 1258(2): 159-168.

Folch, J., M. Lees and G. H. Sloane Stanley (1957). A simple method for the isolation and purification of total lipides from animal tissues. J Biol Chem 226(1): 497-509.

Foretz, M., N. Ancellin, F. Andreelli, Y. Saintillan, P. Grondin, A. Kahn, B. Thorens, S. Vaulont and B. Viollet (2005). Short-term overexpression of a constitutively active form of AMPactivated protein kinase in the liver leads to mild hypoglycemia and fatty liver. Diabetes $\mathbf{5 4}(5)$ : 1331-1339.

Fracanzani, A. L., L. Valenti, E. Bugianesi, M. Andreoletti, A. Colli, E. Vanni, C. Bertelli, E. Fatta, D. Bignamini, G. Marchesini and S. Fargion (2008). Risk of severe liver disease in nonalcoholic fatty liver disease with normal aminotransferase levels: a role for insulin resistance and diabetes. Hepatology 48(3): 792-798.

Fraser, D. A., J. Thoen, A. C. Rustan, O. Forre and J. Kjeldsen-Kragh (1999). Changes in plasma free fatty acid concentrations in rheumatoid arthritis patients during fasting and their effects upon T-lymphocyte proliferation. Rheumatology (Oxford) 38(10): 948-952.

Furumiya, M., K. Inoue, K. Ohta, Y. Hayashi and H. Yuasa (2013). Transcriptional regulation of PCFT by KLF4, HNF4alpha, CDX2 and C/EBPalpha: implication in its site-specific expression in the small intestine. Biochem Biophys Res Commun 431(2): 158-163.

Gan, R. Y. and H. B. Li (2014). Recent progress on liver kinase B1 (LKB1): expression, regulation, downstream signaling and cancer suppressive function. Int J Mol Sci 15(9): 1669816718.

Gao, B., W. I. Jeong and Z. Tian (2008). Liver: An organ with predominant innate immunity. Hepatology 47(2): 729-736. 
Garcia-Casal, M. N., C. Osorio, M. Landaeta, I. Leets, P. Matus, F. Fazzino and E. Marcos (2005). High prevalence of folic acid and vitamin B12 deficiencies in infants, children, adolescents and pregnant women in Venezuela. Eur J Clin Nutr 59(9): 1064-1070.

Gasparini, C. and M. Feldmann (2012). NF-kappaB as a target for modulating inflammatory responses. Curr Pharm Des 18(35): 5735-5745.

Gauthier, M. S., E. L. O'Brien, S. Bigornia, M. Mott, J. M. Cacicedo, X. J. Xu, N. Gokce, C. Apovian and N. Ruderman (2011). Decreased AMP-activated protein kinase activity is associated with increased inflammation in visceral adipose tissue and with whole-body insulin resistance in morbidly obese humans. Biochem Biophys Res Commun 404(1): 382-387.

Glass, L. M., R. C. Dickson, J. C. Anderson, A. A. Suriawinata, J. Putra, B. S. Berk and A. Toor (2015). Total body weight loss of $>/=10 \%$ is associated with improved hepatic fibrosis in patients with nonalcoholic steatohepatitis. Dig Dis Sci 60(4): 1024-1030.

Gliszczynska-Swiglo, A. and M. Muzolf (2007). pH-Dependent radical scavenging activity of folates. J Agric Food Chem 55(20): 8237-8242.

Godbole, V. and D. A. York (1978). Lipogenesis in situ in the genetically obese Zucker fatty rat (fa/fa): role of hyperphagia and hyperinsulinaemia. Diabetologia 14(3): 191-197.

Golay, A. and E. Bobbioni (1997). The role of dietary fat in obesity. Int J Obes Relat Metab Disord 21 Suppl 3: S2-11.

Goldstein, J. L. and M. S. Brown (1984). Progress in understanding the LDL receptor and HMGCoA reductase, two membrane proteins that regulate the plasma cholesterol. J Lipid Res 25(13): 1450-1461.

Gomez-Lechon, M. J., M. T. Donato, J. V. Castell and R. Jover (2004). Human hepatocytes in primary culture: the choice to investigate drug metabolism in man. Curr Drug Metab 5(5): 443462.

Gonen, N. and Y. G. Assaraf (2010). The obligatory intestinal folate transporter PCFT (SLC46A1) is regulated by nuclear respiratory factor 1. J Biol Chem 285(44): 33602-33613.

Gowans, G. J., S. A. Hawley, F. A. Ross and D. G. Hardie (2013). AMP is a true physiological regulator of AMP-activated protein kinase by both allosteric activation and enhancing net phosphorylation. Cell Metab 18(4): 556-566. 
Grimm, I. S., W. Schindler and O. Haluszka (1992). Steatohepatitis and fatal hepatic failure after biliopancreatic diversion. Am J Gastroenterol 87(6): 775-779.

Gugneja, S. and R. C. Scarpulla (1997). Serine phosphorylation within a concise amino-terminal domain in nuclear respiratory factor 1 enhances DNA binding. J Biol Chem 272(30): 1873218739.

Haas, J. T., S. Francque and B. Staels (2016). Pathophysiology and Mechanisms of Nonalcoholic Fatty Liver Disease. Annu Rev Physiol 78: 181-205.

Hadizadeh, F., E. Faghihimani and P. Adibi (2017). Nonalcoholic fatty liver disease: Diagnostic biomarkers. World J Gastrointest Pathophysiol 8(2): 11-26.

Halliwell, B. and M. Whiteman (2004). Measuring reactive species and oxidative damage in vivo and in cell culture: how should you do it and what do the results mean? Br J Pharmacol 142(2): 231-255.

Han, H. S., G. Kang, J. S. Kim, B. H. Choi and S. H. Koo (2016). Regulation of glucose metabolism from a liver-centric perspective. Exp Mol Med 48: e218.

Hannah, W. N., Jr. and S. A. Harrison (2016). Lifestyle and Dietary Interventions in the Management of Nonalcoholic Fatty Liver Disease. Dig Dis Sci 61(5): 1365-1374.

Hardie, D. G. (2004). The AMP-activated protein kinase pathway--new players upstream and downstream. J Cell Sci 117(Pt 23): 5479-5487.

Hardie, D. G. (2007). AMP-activated/SNF1 protein kinases: conserved guardians of cellular energy. Nat Rev Mol Cell Biol 8(10): 774-785.

Hardie, D. G., F. A. Ross and S. A. Hawley (2012). AMPK: a nutrient and energy sensor that maintains energy homeostasis. Nat Rev Mol Cell Biol 13(4): 251-262.

Hardwick, R. N., C. D. Fisher, M. J. Canet, A. D. Lake and N. J. Cherrington (2010). Diversity in antioxidant response enzymes in progressive stages of human nonalcoholic fatty liver disease. Drug Metab Dispos 38(12): 2293-2301.

Hariri, N., R. Gougeon and L. Thibault (2010). A highly saturated fat-rich diet is more obesogenic than diets with lower saturated fat content. Nutr Res 30(9): 632-643. 
Hashimoto, E., M. Taniai and K. Tokushige (2013). Characteristics and diagnosis of NAFLD/NASH. J Gastroenterol Hepatol 28 Suppl 4: 64-70.

Hawley, S. A., M. Davison, A. Woods, S. P. Davies, R. K. Beri, D. Carling and D. G. Hardie (1996). Characterization of the AMP-activated protein kinase kinase from rat liver and identification of threonine 172 as the major site at which it phosphorylates AMP-activated protein kinase. J Biol Chem 271(44): 27879-27887.

Hayden, M. S. and S. Ghosh (2008). Shared principles in NF-kappaB signaling. Cell 132(3): 344-362.

Hayden, M. S. and S. Ghosh (2012). NF-kappaB, the first quarter-century: remarkable progress and outstanding questions. Genes Dev 26(3): 203-234.

Hebbard, L. and J. George (2011). Animal models of nonalcoholic fatty liver disease. Nat Rev Gastroenterol Hepatol 8(1): 35-44.

Hedley, A. A., C. L. Ogden, C. L. Johnson, M. D. Carroll, L. R. Curtin and K. M. Flegal (2004). Prevalence of overweight and obesity among US children, adolescents, and adults, 1999-2002. JAMA 291(23): 2847-2850.

Henning, S. M., M. E. Swendseid, B. T. Ivandic and F. Liao (1997). Vitamins C, E and A and heme oxygenase in rats fed methyl/folate-deficient diets. Free Radic Biol Med 23(6): 936-942.

Herbig, K., E. P. Chiang, L. R. Lee, J. Hills, B. Shane and P. J. Stover (2002). Cytoplasmic serine hydroxymethyltransferase mediates competition between folate-dependent deoxyribonucleotide and S-adenosylmethionine biosyntheses. J Biol Chem 277(41): 3838138389.

Hirsch, S., J. Poniachick, M. Avendano, A. Csendes, P. Burdiles, G. Smok, J. C. Diaz and M. P. de la Maza (2005). Serum folate and homocysteine levels in obese females with non-alcoholic fatty liver. Nutrition 21(2): 137-141.

Horne, D. W., K. A. Reed, J. Hoefs and H. M. Said (1993). 5-Methyltetrahydrofolate transport in basolateral membrane vesicles from human liver. Am J Clin Nutr 58(1): 80-84.

Hotamisligil, G. S. (2006). Inflammation and metabolic disorders. Nature 444(7121): 860-867. 
Hotamisligil, G. S. and E. Erbay (2008). Nutrient sensing and inflammation in metabolic diseases. Nat Rev Immunol 8(12): 923-934.

Hou, X., S. Xu, K. A. Maitland-Toolan, K. Sato, B. Jiang, Y. Ido, F. Lan, K. Walsh, M. Wierzbicki, T. J. Verbeuren, R. A. Cohen and M. Zang (2008). SIRT1 regulates hepatocyte lipid metabolism through activating AMP-activated protein kinase. J Biol Chem 283(29): 2001520026.

Hu, J., B. Wang and N. R. Sahyoun (2016). Application of the Key Events Dose-response Framework to Folate Metabolism. Crit Rev Food Sci Nutr 56(8): 1325-1333.

Huang, R. F., Y. C. Hsu, H. L. Lin and F. L. Yang (2001). Folate depletion and elevated plasma homocysteine promote oxidative stress in rat livers. J Nutr 131(1): 33-38.

Hui, J. M., J. G. Kench, S. Chitturi, A. Sud, G. C. Farrell, K. Byth, P. Hall, M. Khan and J. George (2003). Long-term outcomes of cirrhosis in nonalcoholic steatohepatitis compared with hepatitis C. Hepatology 38(2): 420-427.

Hwang, S. Y., L. K. Sarna, Y. L. Siow and K. O (2013). High-fat diet stimulates hepatic cystathionine beta-synthase and cystathionine gamma-lyase expression. Can J Physiol Pharmacol 91(11): 913-919.

Hwang, S. Y., Y. L. Siow, K. K. Au-Yeung, J. House and K. O (2011). Folic acid supplementation inhibits NADPH oxidase-mediated superoxide anion production in the kidney. Am J Physiol Renal Physiol 300(1): F189-198.

Ikematsu, N., M. L. Dallas, F. A. Ross, R. W. Lewis, J. N. Rafferty, J. A. David, R. Suman, C. Peers, D. G. Hardie and A. M. Evans (2011). Phosphorylation of the voltage-gated potassium channel Kv2.1 by AMP-activated protein kinase regulates membrane excitability. Proc Natl Acad Sci U S A 108(44): 18132-18137.

Imamura, K., T. Ogura, A. Kishimoto, M. Kaminishi and H. Esumi (2001). Cell cycle regulation via 553 phosphorylation by a 5'-AMP activated protein kinase activator, 5-aminoimidazole- 4carboxamide-1-beta-D-ribofuranoside, in a human hepatocellular carcinoma cell line. Biochem Biophys Res Commun 287(2): 562-567.

Institute of Medicine Standing Committee on the Scientific Evaluation of Dietary Reference, I., O. B. V. its Panel on Folate and Choline (1998). The National Academies Collection: Reports funded by National Institutes of Health. Dietary Reference Intakes for Thiamin, Riboflavin, 
Niacin, Vitamin B6, Folate, Vitamin B12, Pantothenic Acid, Biotin, and Choline. Washington (DC), National Academies Press (US)

Ito, M., J. Suzuki, S. Tsujioka, M. Sasaki, A. Gomori, T. Shirakura, H. Hirose, M. Ito, A. Ishihara, H. Iwaasa and A. Kanatani (2007). Longitudinal analysis of murine steatohepatitis model induced by chronic exposure to high-fat diet. Hepatol Res 37(1): 50-57.

Ito, T. and U. Ikeda (2003). Inflammatory cytokines and cardiovascular disease. Curr Drug Targets Inflamm Allergy 2(3): 257-265.

Iyer, R. and S. K. Tomar (2009). Folate: a functional food constituent. J Food Sci 74(9): R114122.

Jacobs, R. L., S. Lingrell, Y. Zhao, G. A. Francis and D. E. Vance (2008). Hepatic CTP:phosphocholine cytidylyltransferase-alpha is a critical predictor of plasma high density lipoprotein and very low density lipoprotein. J Biol Chem 283(4): 2147-2155.

Jaeschke, H. (2000). Reactive oxygen and mechanisms of inflammatory liver injury. $\underline{\mathbf{J}}$ Gastroenterol Hepatol 15(7): 718-724.

Javitt, N. B. (1990). Hep G2 cells as a resource for metabolic studies: lipoprotein, cholesterol, and bile acids. FASEB J 4(2): 161-168.

Jenkins, C. M., D. J. Mancuso, W. Yan, H. F. Sims, B. Gibson and R. W. Gross (2004). Identification, cloning, expression, and purification of three novel human calcium-independent phospholipase A2 family members possessing triacylglycerol lipase and acylglycerol transacylase activities. J Biol Chem 279(47): 48968-48975.

Joshi-Barve, S., S. S. Barve, K. Amancherla, L. Gobejishvili, D. Hill, M. Cave, P. Hote and C. J. McClain (2007). Palmitic acid induces production of proinflammatory cytokine interleukin-8 from hepatocytes. Hepatology 46(3): 823-830.

Joshi, R., S. Adhikari, B. S. Patro, S. Chattopadhyay and T. Mukherjee (2001). Free radical scavenging behavior of folic acid: evidence for possible antioxidant activity. Free Radic Biol Med 30(12): 1390-1399. 
Jurevics, H., J. Hostettler, C. Barrett, P. Morell and A. D. Toews (2000). Diurnal and dietaryinduced changes in cholesterol synthesis correlate with levels of mRNA for HMG-CoA reductase. J Lipid Res 41(7): 1048-1054.

Kaludercic, N., S. Deshwal and F. Di Lisa (2014). Reactive oxygen species and redox compartmentalization. Front Physiol 5: 285.

Kamen, B. A. and A. K. Smith (2004). A review of folate receptor alpha cycling and 5methyltetrahydrofolate accumulation with an emphasis on cell models in vitro. Adv Drug Deliv Rev 56(8): 1085-1097.

Kanuri, G. and I. Bergheim (2013). In vitro and in vivo models of non-alcoholic fatty liver disease (NAFLD). Int J Mol Sci 14(6): 11963-11980.

Kaplan, M. R. and R. D. Simoni (1985). Transport of cholesterol from the endoplasmic reticulum to the plasma membrane. J Cell Biol 101(2): 446-453.

Kessoku, T., Y. Ogawa, M. Yoneda, K. Imajo, Y. Sumida, Y. Eguchi, H. Fujii, H. Hyogo, M. Ono, Y. Suzuki, T. Kawaguchi, K. Chayama, S. Tanaka, K. Fujimoto, K. Anzai, T. Saibara, M. Sata, Y. Itoh, A. Nakajima and T. Okanoue (2014). Simple scoring system for predicting cirrhosis in nonalcoholic fatty liver disease. World J Gastroenterol 20(29): 10108-10114.

Kim, Y. I. (2007). Folic acid fortification and supplementation--good for some but not so good for others. Nutr Rev 65(11): 504-511.

Kim, Y. I., J. W. Miller, K. A. da Costa, M. Nadeau, D. Smith, J. Selhub, S. H. Zeisel and J. B. Mason (1994). Severe folate deficiency causes secondary depletion of choline and phosphocholine in rat liver. J Nutr 124(11): 2197-2203.

Kleiner, D. E. and E. M. Brunt (2012). Nonalcoholic fatty liver disease: pathologic patterns and biopsy evaluation in clinical research. Semin Liver Dis 32(1): 3-13.

Kleiner, D. E., E. M. Brunt, M. Van Natta, C. Behling, M. J. Contos, O. W. Cummings, L. D. Ferrell, Y. C. Liu, M. S. Torbenson, A. Unalp-Arida, M. Yeh, A. J. McCullough, A. J. Sanyal and N. Nonalcoholic Steatohepatitis Clinical Research (2005). Design and validation of a histological scoring system for nonalcoholic fatty liver disease. Hepatology 41(6): 1313-1321.

Klipstein, F. A. and J. Lindenbaum (1965). Folate Deficiency in Chronic Liver Disease. Blood 25: 443-456. 
Kohjima, M., M. Enjoji, N. Higuchi, M. Kato, K. Kotoh, T. Yoshimoto, T. Fujino, M. Yada, R. Yada, N. Harada, R. Takayanagi and M. Nakamuta (2007). Re-evaluation of fatty acid metabolism-related gene expression in nonalcoholic fatty liver disease. Int J Mol Med 20(3): 351-358.

Kolb, A. F. and L. Petrie (2013). Folate deficiency enhances the inflammatory response of macrophages. Mol Immunol 54(2): 164-172.

Kotronen, A., L. E. Johansson, L. M. Johansson, C. Roos, J. Westerbacka, A. Hamsten, R. Bergholm, P. Arkkila, J. Arola, T. Kiviluoto, R. M. Fisher, E. Ehrenborg, M. Orho-Melander, M. Ridderstrale, L. Groop and H. Yki-Jarvinen (2009). A common variant in PNPLA3, which encodes adiponutrin, is associated with liver fat content in humans. Diabetologia 52(6): 10561060 .

Kwon, H., S. Laurent, Y. Tang, H. Zong, P. Vemulapalli and J. E. Pessin (2014). Adipocytespecific IKKbeta signaling suppresses adipose tissue inflammation through an IL-13-dependent paracrine feedback pathway. Cell Rep 9(5): 1574-1583.

Lam, T. Y., S. W. Seto, A. L. Au, C. C. Poon, R. W. Li, H. Y. Lam, W. S. Lau, S. W. Chan, S. M. Ngai, G. P. Leung, S. M. Lee, S. K. Tsui and Y. W. Kwan (2009). Folic acid supplementation modifies beta-adrenoceptor-mediated in vitro lipolysis of obese/diabetic $(+\mathrm{db} /+\mathrm{db})$ mice. Exp Biol Med (Maywood) 234(9): 1047-1055.

Lambert, J. E., M. A. Ramos-Roman, J. D. Browning and E. J. Parks (2014). Increased de novo lipogenesis is a distinct characteristic of individuals with nonalcoholic fatty liver disease. Gastroenterology 146(3): 726-735.

Lan, F., J. M. Cacicedo, N. Ruderman and Y. Ido (2008). SIRT1 modulation of the acetylation status, cytosolic localization, and activity of LKB1. Possible role in AMP-activated protein kinase activation. J Biol Chem 283(41): 27628-27635.

Lanthier, N., O. Molendi-Coste, P. D. Cani, N. van Rooijen, Y. Horsmans and I. A. Leclercq (2011). Kupffer cell depletion prevents but has no therapeutic effect on metabolic and inflammatory changes induced by a high-fat diet. Faseb j 25(12): 4301-4311.

Larsen, G. L. and P. M. Henson (1983). Mediators of inflammation. Annu Rev Immunol 1: 335359.

Larter, C. Z. and M. M. Yeh (2008). Animal models of NASH: getting both pathology and metabolic context right. J Gastroenterol Hepatol 23(11): 1635-1648. 
Lawrence, T. and D. W. Gilroy (2007). Chronic inflammation: a failure of resolution? Int J Exp Pathol 88(2): 85-94.

Lawrence, T., D. W. Gilroy, P. R. Colville-Nash and D. A. Willoughby (2001). Possible new role for NF-kappaB in the resolution of inflammation. Nat Med 7(12): 1291-1297.

Leamy, A. K., R. A. Egnatchik and J. D. Young (2013). Molecular mechanisms and the role of saturated fatty acids in the progression of non-alcoholic fatty liver disease. Prog Lipid Res 52(1): 165-174.

Leblanc, M., V. Pichette, D. Geadah and D. Ouimet (2000). Folic acid and pyridoxal-5'phosphate losses during high-efficiency hemodialysis in patients without hydrosoluble vitamin supplementation. J Ren Nutr 10(4): 196-201.

Leevy, C. M. and H. Baker (1970). Nutritional deficiencies in liver disease. Med Clin North Am 54(2): 467-477.

Leevy, C. M., A. Thompson and H. Baker (1970). Vitamins and liver injury. Am J Clin Nutr 23(4): 493-499.

Levin, R. J. (1994). Digestion and absorption of carbohydrates--from molecules and membranes to humans. Am J Clin Nutr 59(3 Suppl): 690s-698s.

Li, Z. and D. E. Vance (2008). Phosphatidylcholine and choline homeostasis. J Lipid Res 49(6): 1187-1194.

Ling, J., J. Lewis, D. Douglas, N. M. Kneteman and D. E. Vance (2013). Characterization of lipid and lipoprotein metabolism in primary human hepatocytes. Biochim Biophys Acta 1831(2): 387-397.

Liu, M., Y. Ge, D. C. Cabelof, A. Aboukameel, A. R. Heydari, R. Mohammad and L. H. Matherly (2005). Structure and regulation of the murine reduced folate carrier gene: identification of four noncoding exons and promoters and regulation by dietary folates. $\underline{\mathrm{J} \text { Biol }}$ Chem 280(7): 5588-5597.

Liu, M., J. R. Whetstine, S. G. Payton, Y. Ge, R. M. Flatley and L. H. Matherly (2004). Roles of USF, Ikaros and $\mathrm{Sp}$ proteins in the transcriptional regulation of the human reduced folate carrier B promoter. Biochem J 383(Pt 2): 249-257. 
Lochhead, P. A., I. P. Salt, K. S. Walker, D. G. Hardie and C. Sutherland (2000). 5aminoimidazole-4-carboxamide riboside mimics the effects of insulin on the expression of the 2 key gluconeogenic genes PEPCK and glucose-6-phosphatase. Diabetes 49(6): 896-903.

Loguercio, C., T. De Simone, M. V. D'Auria, I. de Sio, A. Federico, C. Tuccillo, A. M. Abbatecola, C. Del Vecchio Blanco and A. C. G. Italian (2004). Non-alcoholic fatty liver disease: a multicentre clinical study by the Italian Association for the Study of the Liver. Dig Liver Dis 36(6): 398-405.

Lonn, E., S. Yusuf, M. J. Arnold, P. Sheridan, J. Pogue, M. Micks, M. J. McQueen, J. Probstfield, G. Fodor, C. Held and J. Genest, Jr. (2006). Homocysteine lowering with folic acid and B vitamins in vascular disease. $\mathrm{N} \mathrm{Engl} \mathrm{J} \mathrm{Med} \mathrm{354(15):} \mathrm{1567-1577.}$

Loomba, R. and A. J. Sanyal (2013). The global NAFLD epidemic. Nat Rev Gastroenterol Hepatol 10(11): 686-690.

Lu, K., M. H. Lee and S. B. Patel (2001). Dietary cholesterol absorption; more than just bile. Trends Endocrinol Metab 12(7): 314-320.

Lucock, M. (2000). Folic acid: nutritional biochemistry, molecular biology, and role in disease processes. Mol Genet Metab 71(1-2): 121-138.

Ludwig, J., T. R. Viggiano, D. B. McGill and B. J. Oh (1980). Nonalcoholic steatohepatitis: Mayo Clinic experiences with a hitherto unnamed disease. Mayo Clin Proc 55(7): 434-438.

Lumeng, C. N. and A. R. Saltiel (2011). Inflammatory links between obesity and metabolic disease. J Clin Invest 121(6): 2111-2117.

Luo, Y., C. M. Burrington, E. C. Graff, J. Zhang, R. L. Judd, P. Suksaranjit, Q. Kaewpoowat, S. K. Davenport, A. M. O'Neill and M. W. Greene (2016). Metabolic phenotype and adipose and liver features in a high-fat Western diet-induced mouse model of obesity-linked NAFLD. Am J Physiol Endocrinol Metab 310(6): E418-439.

Luster, A. D., R. Alon and U. H. von Andrian (2005). Immune cell migration in inflammation: present and future therapeutic targets. Nat Immunol 6(12): 1182-1190.

Magoulas, P. L. and A. W. El-Hattab (2012). Systemic primary carnitine deficiency: an overview of clinical manifestations, diagnosis, and management. Orphanet J Rare Dis 7: 68. 
Mahabir, S., S. Ettinger, L. Johnson, D. J. Baer, B. A. Clevidence, T. J. Hartman and P. R. Taylor (2008). Measures of adiposity and body fat distribution in relation to serum folate levels in postmenopausal women in a feeding study. Eur J Clin Nutr 62(5): 644-650.

Malaguarnera, M., M. P. Gargante, C. Russo, T. Antic, M. Vacante, M. Malaguarnera, T. Avitabile, G. Li Volti and F. Galvano (2010). L-carnitine supplementation to diet: a new tool in treatment of nonalcoholic steatohepatitis--a randomized and controlled clinical trial. Am J Gastroenterol 105(6): 1338-1345.

Marchesini, G., M. Brizi, G. Bianchi, S. Tomassetti, E. Bugianesi, M. Lenzi, A. J. McCullough, S. Natale, G. Forlani and N. Melchionda (2001). Nonalcoholic fatty liver disease: a feature of the metabolic syndrome. Diabetes 50(8): 1844-1850.

Marchesini, G., M. Brizi, A. M. Morselli-Labate, G. Bianchi, E. Bugianesi, A. J. McCullough, G. Forlani and N. Melchionda (1999). Association of nonalcoholic fatty liver disease with insulin resistance. Am J Med 107(5): 450-455.

Marchesini, G., E. Bugianesi, G. Forlani, F. Cerrelli, M. Lenzi, R. Manini, S. Natale, E. Vanni, N. Villanova, N. Melchionda and M. Rizzetto (2003). Nonalcoholic fatty liver, steatohepatitis, and the metabolic syndrome. Hepatology 37(4): 917-923.

Martin, P. and S. J. Leibovich (2005). Inflammatory cells during wound repair: the good, the bad and the ugly. Trends Cell Biol 15(11): 599-607.

Matherly, L. H., Z. Hou and Y. Deng (2007). Human reduced folate carrier: translation of basic biology to cancer etiology and therapy. Cancer Metastasis Rev 26(1): 111-128.

Mayer, J., M. W. Bates and M. M. Dickie (1951). Hereditary diabetes in genetically obese mice. Science 113(2948): 746-747.

McLaren, J. E., D. R. Michael, T. G. Ashlin and D. P. Ramji (2011). Cytokines, macrophage lipid metabolism and foam cells: implications for cardiovascular disease therapy. Prog Lipid Res 50(4): 331-347.

McNeil, C. J., S. M. Hay, G. J. Rucklidge, M. Reid, G. Duncan, C. A. Maloney and W. D. Rees (2008). Disruption of lipid metabolism in the liver of the pregnant rat fed folate-deficient and methyl donor-deficient diets. Br J Nutr 99(2): 262-271. 
Mechanick, J. I., R. F. Kushner, H. J. Sugerman, J. M. Gonzalez-Campoy, M. L. CollazoClavell, A. F. Spitz, C. M. Apovian, E. H. Livingston, R. Brolin, D. B. Sarwer, W. A. Anderson, J. Dixon and S. Guven (2009). American Association of Clinical Endocrinologists, The Obesity Society, and American Society for Metabolic \& Bariatric Surgery medical guidelines for clinical practice for the perioperative nutritional, metabolic, and nonsurgical support of the bariatric surgery patient. Obesity (Silver Spring) 17 Suppl 1: S1-70, v.

Medici, V. and C. H. Halsted (2013). Folate, alcohol, and liver disease. Mol Nutr Food Res 57(4): 596-606.

Miele, L., V. Valenza, G. La Torre, M. Montalto, G. Cammarota, R. Ricci, R. Masciana, A. Forgione, M. L. Gabrieli, G. Perotti, F. M. Vecchio, G. Rapaccini, G. Gasbarrini, C. P. Day and A. Grieco (2009). Increased intestinal permeability and tight junction alterations in nonalcoholic fatty liver disease. Hepatology 49(6): 1877-1887.

Min, H. K., A. Kapoor, M. Fuchs, F. Mirshahi, H. Zhou, J. Maher, J. Kellum, R. Warnick, M. J. Contos and A. J. Sanyal (2012). Increased hepatic synthesis and dysregulation of cholesterol metabolism is associated with the severity of nonalcoholic fatty liver disease. Cell Metab 15(5): 665-674.

Miranda, T. B. and P. A. Jones (2007). DNA methylation: the nuts and bolts of repression. J Cell Physiol 213(2): 384-390.

Mojtabai, R. (2004). Body mass index and serum folate in childbearing age women. Eur J Epidemiol 19(11): 1029-1036.

Monetti, M., M. C. Levin, M. J. Watt, M. P. Sajan, S. Marmor, B. K. Hubbard, R. D. Stevens, J. R. Bain, C. B. Newgard, R. V. Farese, Sr., A. L. Hevener and R. V. Farese, Jr. (2007). Dissociation of hepatic steatosis and insulin resistance in mice overexpressing DGAT in the liver. Cell Metab 6(1): 69-78.

Morris, M. S., P. F. Jacques, I. H. Rosenberg and J. Selhub (2010). Circulating unmetabolized folic acid and 5-methyltetrahydrofolate in relation to anemia, macrocytosis, and cognitive test performance in American seniors. Am J Clin Nutr 91(6): 1733-1744.

Must, A. and N. M. McKeown (2000). The Disease Burden Associated with Overweight and Obesity. Endotext. South Dartmouth (MA). 
Neuschwander-Tetri, B. A. (2010). Hepatic lipotoxicity and the pathogenesis of nonalcoholic steatohepatitis: the central role of nontriglyceride fatty acid metabolites. Hepatology 52(2): 774788.

Neuschwander-Tetri, B. A., E. M. Brunt, K. R. Wehmeier, D. Oliver and B. R. Bacon (2003). Improved nonalcoholic steatohepatitis after 48 weeks of treatment with the PPAR-gamma ligand rosiglitazone. Hepatology 38(4): 1008-1017.

Newberry, E. P., Y. Xie, S. Kennedy, X. Han, K. K. Buhman, J. Luo, R. W. Gross and N. O. Davidson (2003). Decreased hepatic triglyceride accumulation and altered fatty acid uptake in mice with deletion of the liver fatty acid-binding protein gene. J Biol Chem 278(51): 5166451672 .

Nilsson, E., A. Matte, A. Perfilyev, V. D. de Mello, P. Kakela, J. Pihlajamaki and C. Ling (2015). Epigenetic Alterations in Human Liver From Subjects With Type 2 Diabetes in Parallel With Reduced Folate Levels. J Clin Endocrinol Metab 100(11): E1491-1501.

Noga, A. A. and D. E. Vance (2003). A gender-specific role for phosphatidylethanolamine Nmethyltransferase-derived phosphatidylcholine in the regulation of plasma high density and very low density lipoproteins in mice. J Biol Chem 278(24): 21851-21859.

Nordlie, R. C., J. D. Foster and A. J. Lange (1999). Regulation of glucose production by the liver. Annu Rev Nutr 19: 379-406.

O'Neill, L. A., D. Golenbock and A. G. Bowie (2013). The history of Toll-like receptors redefining innate immunity. Nat Rev Immunol 13(6): 453-460.

Oana, F., H. Takeda, K. Hayakawa, A. Matsuzawa, S. Akahane, M. Isaji and M. Akahane (2005). Physiological difference between obese (fa/fa) Zucker rats and lean Zucker rats concerning adiponectin. Metabolism 54(8): 995-1001.

Omagari, K., S. Kato, K. Tsuneyama, C. Inohara, Y. Kuroda, H. Tsukuda, E. Fukazawa, K. Shiraishi and M. Mune (2008). Effects of a long-term high-fat diet and switching from a high-fat to low-fat, standard diet on hepatic fat accumulation in Sprague-Dawley rats. Dig Dis Sci 53(12): 3206-3212.

Omkumar, R. V., B. G. Darnay and V. W. Rodwell (1994). Modulation of Syrian hamster 3hydroxy-3-methylglutaryl-CoA reductase activity by phosphorylation. Role of serine 871 . J Biol Chem 269(9): 6810-6814. 
Park, E. J., J. H. Lee, G. Y. Yu, G. He, S. R. Ali, R. G. Holzer, C. H. Osterreicher, H. Takahashi and M. Karin (2010). Dietary and genetic obesity promote liver inflammation and tumorigenesis by enhancing IL-6 and TNF expression. Cell 140(2): 197-208.

Perry, J. (1971). Folate analogues in normal mixed diets. Br J Haematol 21(4): 435-441.

Petersen, K. F., S. Dufour, A. Hariri, C. Nelson-Williams, J. N. Foo, X. M. Zhang, J. Dziura, R. P. Lifton and G. I. Shulman (2010). Apolipoprotein C3 gene variants in nonalcoholic fatty liver disease. N Engl J Med 362(12): 1082-1089.

Piedrahita, J. A., B. Oetama, G. D. Bennett, J. van Waes, B. A. Kamen, J. Richardson, S. W. Lacey, R. G. Anderson and R. H. Finnell (1999). Mice lacking the folic acid-binding protein Folbp1 are defective in early embryonic development. Nat Genet 23(2): 228-232.

Pietrzik, K., L. Bailey and B. Shane (2010). Folic acid and L-5-methyltetrahydrofolate: comparison of clinical pharmacokinetics and pharmacodynamics. Clin Pharmacokinet 49(8): 535-548.

Pike, S. T., R. Rajendra, K. Artzt and D. R. Appling (2010). Mitochondrial C1-tetrahydrofolate synthase (MTHFD1L) supports the flow of mitochondrial one-carbon units into the methyl cycle in embryos. J Biol Chem 285(7): 4612-4620.

Pu, P., D. M. Gao, S. Mohamed, J. Chen, J. Zhang, X. Y. Zhou, N. J. Zhou, J. Xie and H. Jiang (2012). Naringin ameliorates metabolic syndrome by activating AMP-activated protein kinase in mice fed a high-fat diet. Arch Biochem Biophys 518(1): 61-70.

Puri, P., R. A. Baillie, M. M. Wiest, F. Mirshahi, J. Choudhury, O. Cheung, C. Sargeant, M. J. Contos and A. J. Sanyal (2007). A lipidomic analysis of nonalcoholic fatty liver disease.

Hepatology 46(4): 1081-1090.

Purohit, V., M. F. Abdelmalek, S. Barve, N. J. Benevenga, C. H. Halsted, N. Kaplowitz, K. K. Kharbanda, Q. Y. Liu, S. C. Lu, C. J. McClain, C. Swanson and S. Zakhari (2007). Role of Sadenosylmethionine, folate, and betaine in the treatment of alcoholic liver disease: summary of a symposium. Am J Clin Nutr 86(1): 14-24.

Qiu, A., M. Jansen, A. Sakaris, S. H. Min, S. Chattopadhyay, E. Tsai, C. Sandoval, R. Zhao, M. H. Akabas and I. D. Goldman (2006). Identification of an intestinal folate transporter and the molecular basis for hereditary folate malabsorption. Cell 127(5): 917-928. 
Racanelli, V. and B. Rehermann (2006). The liver as an immunological organ. Hepatology 43(2 Suppl 1): S54-62.

Rafiq, N., C. Bai, Y. Fang, M. Srishord, A. McCullough, T. Gramlich and Z. M. Younossi (2009). Long-term follow-up of patients with nonalcoholic fatty liver. Clin Gastroenterol Hepatol 7(2): 234-238.

Raghunathan, K., J. C. Schmitz and D. G. Priest (1997). Disposition of leucovorin and its metabolites in dietary folic acid-deplete mice - comparison between tumor, liver, and plasma. Cancer Chemother Pharmacol 40(2): 126-130.

Rahman, K., C. Desai, S. S. Iyer, N. E. Thorn, P. Kumar, Y. Liu, T. Smith, A. S. Neish, H. Li, S. Tan, P. Wu, X. Liu, Y. Yu, A. B. Farris, A. Nusrat, C. A. Parkos and F. A. Anania (2016). Loss of Junctional Adhesion Molecule A Promotes Severe Steatohepatitis in Mice on a Diet High in Saturated Fat, Fructose, and Cholesterol. Gastroenterology 151(4): 733-746.e712.

Ratziu, V., F. Charlotte, A. Heurtier, S. Gombert, P. Giral, E. Bruckert, A. Grimaldi, F. Capron and T. Poynard (2005). Sampling variability of liver biopsy in nonalcoholic fatty liver disease. Gastroenterology 128(7): 1898-1906.

Reddy, J. K. and M. S. Rao (2006). Lipid metabolism and liver inflammation. II. Fatty liver disease and fatty acid oxidation. Am J Physiol Gastrointest Liver Physiol 290(5): G852-858.

Regimbeau, J. M., M. Colombat, P. Mognol, F. Durand, E. Abdalla, C. Degott, F. Degos, O. Farges and J. Belghiti (2004). Obesity and diabetes as a risk factor for hepatocellular carcinoma. Liver Transpl 10(2 Suppl 1): S69-73.

Rencurel, F., G. Waeber, B. Antoine, F. Rocchiccioli, P. Maulard, J. Girard and A. Leturque (1996). Requirement of glucose metabolism for regulation of glucose transporter type 2 (GLUT2) gene expression in liver. Biochem J 314 ( Pt 3): 903-909.

Ribeiro, P. S., H. Cortez-Pinto, S. Sola, R. E. Castro, R. M. Ramalho, A. Baptista, M. C. Moura, M. E. Camilo and C. M. Rodrigues (2004). Hepatocyte apoptosis, expression of death receptors, and activation of NF-kappaB in the liver of nonalcoholic and alcoholic steatohepatitis patients. Am J Gastroenterol 99(9): 1708-1717.

Rinella, M. E., M. S. Elias, R. R. Smolak, T. Fu, J. Borensztajn and R. M. Green (2008). Mechanisms of hepatic steatosis in mice fed a lipogenic methionine choline-deficient diet. $\underline{\mathrm{J}}$ Lipid Res 49(5): 1068-1076. 
Rolo, A. P., J. S. Teodoro and C. M. Palmeira (2012). Role of oxidative stress in the pathogenesis of nonalcoholic steatohepatitis. Free Radic Biol Med 52(1): 59-69.

Romeo, S., J. Kozlitina, C. Xing, A. Pertsemlidis, D. Cox, L. A. Pennacchio, E. Boerwinkle, J. C. Cohen and H. H. Hobbs (2008). Genetic variation in PNPLA3 confers susceptibility to nonalcoholic fatty liver disease. Nat Genet 40(12): 1461-1465.

Rong, N., J. Selhub, B. R. Goldin and I. H. Rosenberg (1991). Bacterially synthesized folate in rat large intestine is incorporated into host tissue folyl polyglutamates. J Nutr 121(12): 19551959.

Ros, E. (2000). Intestinal absorption of triglyceride and cholesterol. Dietary and pharmacological inhibition to reduce cardiovascular risk. Atherosclerosis 151(2): 357-379.

Rui, L. (2014). Energy metabolism in the liver. Compr Physiol 4(1): 177-197.

Saha, A. K., X. J. Xu, E. Lawson, R. Deoliveira, A. E. Brandon, E. W. Kraegen and N. B. Ruderman (2010). Downregulation of AMPK accompanies leucine- and glucose-induced increases in protein synthesis and insulin resistance in rat skeletal muscle. Diabetes 59(10): 2426-2434.

Salazar, M. D. and M. Ratnam (2007). The folate receptor: what does it promise in tissuetargeted therapeutics? Cancer Metastasis Rev 26(1): 141-152.

Saltiel, A. R. and C. R. Kahn (2001). Insulin signalling and the regulation of glucose and lipid metabolism. Nature 414(6865): 799-806.

Sanyal, A. J., C. Campbell-Sargent, F. Mirshahi, W. B. Rizzo, M. J. Contos, R. K. Sterling, V. A. Luketic, M. L. Shiffman and J. N. Clore (2001). Nonalcoholic steatohepatitis: association of insulin resistance and mitochondrial abnormalities. Gastroenterology 120(5): 1183-1192.

Sanyal, A. J., P. S. Mofrad, M. J. Contos, C. Sargeant, V. A. Luketic, R. K. Sterling, R. T. Stravitz, M. L. Shiffman, J. Clore and A. S. Mills (2004). A pilot study of vitamin E versus vitamin $\mathrm{E}$ and pioglitazone for the treatment of nonalcoholic steatohepatitis. Clin Gastroenterol Hepatol 2(12): 1107-1115.

Sarna, L. K., N. Wu, P. Wang, S. Y. Hwang, Y. L. Siow and K. O (2012). Folic acid supplementation attenuates high fat diet induced hepatic oxidative stress via regulation of NADPH oxidase. Can J Physiol Pharmacol 90(2): 155-165. 
Satapati, S., B. Kucejova, J. A. Duarte, J. A. Fletcher, L. Reynolds, N. E. Sunny, T. He, L. A. Nair, K. A. Livingston, X. Fu, M. E. Merritt, A. D. Sherry, C. R. Malloy, J. M. Shelton, J. Lambert, E. J. Parks, I. Corbin, M. A. Magnuson, J. D. Browning and S. C. Burgess (2015). Mitochondrial metabolism mediates oxidative stress and inflammation in fatty liver. $\mathrm{J}$ Clin Invest 125(12): 4447-4462.

Sato, A., H. Kawano, T. Notsu, M. Ohta, M. Nakakuki, K. Mizuguchi, M. Itoh, T. Suganami and Y. Ogawa (2010). Antiobesity effect of eicosapentaenoic acid in high-fat/high-sucrose dietinduced obesity: importance of hepatic lipogenesis. Diabetes 59(10): 2495-2504.

Sawyer, D. E. and B. Van Houten (1999). Repair of DNA damage in mitochondria. Mutat Res 434(3): 161-176.

Schattenberg, J. M., M. Schuchmann and P. R. Galle (2011). Cell death and hepatocarcinogenesis: Dysregulation of apoptosis signaling pathways. J Gastroenterol Hepatol 26 Suppl 1: 213-219.

Schuppan, D. and J. M. Schattenberg (2013). Non-alcoholic steatohepatitis: pathogenesis and novel therapeutic approaches. J Gastroenterol Hepatol 28 Suppl 1: 68-76.

Schwimmer, J. B., N. McGreal, R. Deutsch, M. J. Finegold and J. E. Lavine (2005). Influence of gender, race, and ethnicity on suspected fatty liver in obese adolescents. Pediatrics 115(5): e561565.

Scott, J. and D. Weir (1994). Folate/vitamin B12 inter-relationships. Essays Biochem 28: 63-72.

Sehayek, E., R. Wang, J. G. Ono, V. S. Zinchuk, E. M. Duncan, S. Shefer, D. E. Vance, M. Ananthanarayanan, B. T. Chait and J. L. Breslow (2003). Localization of the PE methylation pathway and SR-BI to the canalicular membrane: evidence for apical PC biosynthesis that may promote biliary excretion of phospholipid and cholesterol. J Lipid Res 44(9): 1605-1613.

Seki, S., T. Kitada, T. Yamada, H. Sakaguchi, K. Nakatani and K. Wakasa (2002). In situ detection of lipid peroxidation and oxidative DNA damage in non-alcoholic fatty liver diseases. $\underline{\mathbf{J}}$ Hepatol 37(1): 56-62.

Semple, R. K., A. Sleigh, P. R. Murgatroyd, C. A. Adams, L. Bluck, S. Jackson, A. Vottero, D. Kanabar, V. Charlton-Menys, P. Durrington, M. A. Soos, T. A. Carpenter, D. J. Lomas, E. K. Cochran, P. Gorden, S. O'Rahilly and D. B. Savage (2009). Postreceptor insulin resistance contributes to human dyslipidemia and hepatic steatosis. J Clin Invest 119(2): 315-322. 
Shafizadeh, T. B. and C. H. Halsted (2007). gamma-Glutamyl hydrolase, not glutamate carboxypeptidase II, hydrolyzes dietary folate in rat small intestine. J Nutr 137(5): 1149-1153.

Shang, Y., E. Khafipour, H. Derakhshani, L. K. Sarna, C. W. Woo, Y. L. Siow and K. O (2017). Short Term High Fat Diet Induces Obesity-Enhancing Changes in Mouse Gut Microbiota That are Partially Reversed by Cessation of the High Fat Diet. Lipids 52(6): 499-511.

Sharifnia, T., J. Antoun, T. G. Verriere, G. Suarez, J. Wattacheril, K. T. Wilson, R. M. Peek, Jr., N. N. Abumrad and C. R. Flynn (2015). Hepatic TLR4 signaling in obese NAFLD. Am J Physiol Gastrointest Liver Physiol 309(4): G270-278.

Shaw, R. J., K. A. Lamia, D. Vasquez, S. H. Koo, N. Bardeesy, R. A. Depinho, M. Montminy and L. C. Cantley (2005). The kinase LKB1 mediates glucose homeostasis in liver and therapeutic effects of metformin. Science 310(5754): 1642-1646.

Shiau, Y. F. (1981). Mechanisms of intestinal fat absorption. Am J Physiol 240(1): G1-9.

Shin, Y. S., C. Chan, A. J. Vidal, T. Brody and E. L. Stokstad (1976). Subcellular localization of gamma-glutamyl carboxypeptidase and of folates. Biochim Biophys Acta 444(3): 794-801.

Sid, V., Y. L. Siow and K. O (2017). Role of folate in nonalcoholic fatty liver disease. Can J Physiol Pharmacol 95(10): 1141-1148.

Sid, V., N. Wu, L. K. Sarna, Y. L. Siow, J. D. House and K. O (2015). Folic acid supplementation during high-fat diet feeding restores AMPK activation via an AMP-LKB1dependent mechanism. Am J Physiol Regul Integr Comp Physiol 309(10): R1215-1225.

Simonen, P., A. Kotronen, M. Hallikainen, K. Sevastianova, J. Makkonen, A. Hakkarainen, N. Lundbom, T. A. Miettinen, H. Gylling and H. Yki-Jarvinen (2011). Cholesterol synthesis is increased and absorption decreased in non-alcoholic fatty liver disease independent of obesity. $\underline{\mathbf{J}}$ Hepatol 54(1): 153-159.

Smith, B. K., K. Marcinko, E. M. Desjardins, J. S. Lally, R. J. Ford and G. R. Steinberg (2016). Treatment of nonalcoholic fatty liver disease: role of AMPK. Am J Physiol Endocrinol Metab 311(4): E730-e740.

Softic, S., D. E. Cohen and C. R. Kahn (2016). Role of Dietary Fructose and Hepatic De Novo Lipogenesis in Fatty Liver Disease. Dig Dis Sci 61(5): 1282-1293. 
Solini, A., E. Santini and E. Ferrannini (2006). Effect of short-term folic acid supplementation on insulin sensitivity and inflammatory markers in overweight subjects. Int J Obes (Lond) $\mathbf{3 0}(8)$ :

1197-1202.

Song, P., Z. Xie, Y. Wu, J. Xu, Y. Dong and M. H. Zou (2008). Protein kinase Czeta-dependent LKB1 serine 428 phosphorylation increases LKB1 nucleus export and apoptosis in endothelial cells. J Biol Chem 283(18): 12446-12455.

Sookoian, S. and C. J. Pirola (2012). PNPLA3, the triacylglycerol synthesis/hydrolysis/storage dilemma, and nonalcoholic fatty liver disease. World J Gastroenterol 18(42): 6018-6026.

Spruss, A., G. Kanuri, K. Uebel, S. C. Bischoff and I. Bergheim (2011). Role of the inducible nitric oxide synthase in the onset of fructose-induced steatosis in mice. Antioxid Redox Signal 14(11): 2121-2135.

Starley, B. Q., C. J. Calcagno and S. A. Harrison (2010). Nonalcoholic fatty liver disease and hepatocellular carcinoma: a weighty connection. Hepatology 51(5): 1820-1832.

Stein, S. C., A. Woods, N. A. Jones, M. D. Davison and D. Carling (2000). The regulation of AMP-activated protein kinase by phosphorylation. Biochem J 345 Pt 3: 437-443.

Steinberg, G. R. and B. E. Kemp (2009). AMPK in Health and Disease. Physiol Rev 89(3): 1025-1078.

Steinberg, S. E. (1984). Mechanisms of folate homeostasis. Am J Physiol 246(4 Pt 1): G319-324.

Steinberg, S. E., C. L. Campbell and R. S. Hillman (1979). Kinetics of the normal folate enterohepatic cycle. J Clin Invest 64(1): 83-88.

Stevens, B. R., J. D. Kaunitz and E. M. Wright (1984). Intestinal transport of amino acids and sugars: advances using membrane vesicles. Annu Rev Physiol 46: 417-433.

Stover, P. J. (2004). Physiology of folate and vitamin B12 in health and disease. Nutr Rev $62(6$ Pt 2): S3-12; discussion S13.

Stover, P. J. and M. S. Field (2011). Trafficking of intracellular folates. Adv Nutr 2(4): 325-331. 
Sutti, S., A. Jindal, I. Locatelli, M. Vacchiano, L. Gigliotti, C. Bozzola and E. Albano (2014). Adaptive immune responses triggered by oxidative stress contribute to hepatic inflammation in NASH. Hepatology 59(3): 886-897.

Takahashi, Y., Y. Soejima and T. Fukusato (2012). Animal models of nonalcoholic fatty liver disease/nonalcoholic steatohepatitis. World J Gastroenterol 18(19): 2300-2308.

Tandra, S., M. M. Yeh, E. M. Brunt, R. Vuppalanchi, O. W. Cummings, A. Unalp-Arida, L. A. Wilson and N. Chalasani (2011). Presence and significance of microvesicular steatosis in nonalcoholic fatty liver disease. J Hepatol 55(3): 654-659.

Thoma, C., C. P. Day and M. I. Trenell (2012). Lifestyle interventions for the treatment of nonalcoholic fatty liver disease in adults: a systematic review. J Hepatol 56(1): 255-266.

Tibbetts, A. S. and D. R. Appling (2010). Compartmentalization of Mammalian folate-mediated one-carbon metabolism. Annu Rev Nutr 30: 57-81.

Tilg, H. and A. R. Moschen (2010). Evolution of inflammation in nonalcoholic fatty liver disease: the multiple parallel hits hypothesis. Hepatology 52(5): 1836-1846.

Tiniakos, D. G., M. B. Vos and E. M. Brunt (2010). Nonalcoholic fatty liver disease: pathology and pathogenesis. Annu Rev Pathol 5: 145-171.

Unal, E. S., R. Zhao, A. Qiu and I. D. Goldman (2008). N-linked glycosylation and its impact on the electrophoretic mobility and function of the human proton-coupled folate transporter (HsPCFT). Biochim Biophys Acta 1778(6): 1407-1414.

Updegraff, T. A. and N. J. Neufeld (1981). Protein, iron, and folate status of patients prior to and following surgery for morbid obesity. J Am Diet Assoc 78(2): 135-140.

Utzschneider, K. M. and S. E. Kahn (2006). Review: The role of insulin resistance in nonalcoholic fatty liver disease. J Clin Endocrinol Metab 91(12): 4753-4761.

Uygun, A., A. Kadayifci, Z. Yesilova, A. Erdil, H. Yaman, M. Saka, M. S. Deveci, S. Bagci, M. Gulsen, N. Karaeren and K. Dagalp (2000). Serum leptin levels in patients with nonalcoholic steatohepatitis. Am J Gastroenterol 95(12): 3584-3589. 
Vance, D. E., Z. Li and R. L. Jacobs (2007). Hepatic phosphatidylethanolamine Nmethyltransferase, unexpected roles in animal biochemistry and physiology. J Biol Chem 282(46): 33237-33241.

Veena, J., A. Muragundla, S. Sidgiddi and S. Subramaniam (2014). Non-alcoholic fatty liver disease: need for a balanced nutritional source. Br J Nutr 112(11): 1858-1872.

Vernon, G., A. Baranova and Z. M. Younossi (2011). Systematic review: the epidemiology and natural history of non-alcoholic fatty liver disease and non-alcoholic steatohepatitis in adults. Aliment Pharmacol Ther 34(3): 274-285.

Videla, L. A., R. Rodrigo, M. Orellana, V. Fernandez, G. Tapia, L. Quinones, N. Varela, J. Contreras, R. Lazarte, A. Csendes, J. Rojas, F. Maluenda, P. Burdiles, J. C. Diaz, G. Smok, L. Thielemann and J. Poniachik (2004). Oxidative stress-related parameters in the liver of nonalcoholic fatty liver disease patients. Clin Sci (Lond) 106(3): 261-268.

Vilar, L., C. P. Oliveira, J. Faintuch, E. S. Mello, M. A. Nogueira, T. E. Santos, V. A. Alves and F. J. Carrilho (2008). High-fat diet: a trigger of non-alcoholic steatohepatitis? Preliminary findings in obese subjects. Nutrition 24(11-12): 1097-1102.

Viollet, B., F. Andreelli, S. B. Jorgensen, C. Perrin, A. Geloen, D. Flamez, J. Mu, C. Lenzner, O. Baud, M. Bennoun, E. Gomas, G. Nicolas, J. F. Wojtaszewski, A. Kahn, D. Carling, F. C. Schuit, M. J. Birnbaum, E. A. Richter, R. Burcelin and S. Vaulont (2003). The AMP-activated protein kinase alpha 2 catalytic subunit controls whole-body insulin sensitivity. J Clin Invest 111(1): 91-98.

Viollet, B., M. Foretz, B. Guigas, S. Horman, R. Dentin, L. Bertrand, L. Hue and F. Andreelli (2006). Activation of AMP-activated protein kinase in the liver: a new strategy for the management of metabolic hepatic disorders. J Physiol 574(Pt 1): 41-53.

Viollet, B., B. Guigas, J. Leclerc, S. Hebrard, L. Lantier, R. Mounier, F. Andreelli and M. Foretz (2009). AMP-activated protein kinase in the regulation of hepatic energy metabolism: from physiology to therapeutic perspectives. Acta Physiol (Oxf) 196(1): 81-98.

Visentin, M., N. Diop-Bove, R. Zhao and I. D. Goldman (2014). The intestinal absorption of folates. Annu Rev Physiol 76: 251-274.

Visentin, M., R. Zhao and I. D. Goldman (2012). Augmentation of reduced folate carriermediated folate/antifolate transport through an antiport mechanism with 5-aminoimidazole-4carboxamide riboside monophosphate. Mol Pharmacol 82(2): 209-216. 
Wald, D. S., M. Law and J. K. Morris (2002). Homocysteine and cardiovascular disease: evidence on causality from a meta-analysis. BMJ 325(7374): 1202.

Wang, G., J. Dai, J. Mao, X. Zeng, X. Yang and X. Wang (2005). Folic acid reverses hyperresponsiveness of LPS-induced chemokine secretion from monocytes in patients with hyperhomocysteinemia. Atherosclerosis 179(2): 395-402.

Wang, Y., R. Zhao, R. G. Russell and I. D. Goldman (2001). Localization of the murine reduced folate carrier as assessed by immunohistochemical analysis. Biochim Biophys Acta 1513(1): 4954.

Wang, Y. C., Y. M. Chen, Y. J. Lin, S. P. Liu and E. P. Chiang (2011). GNMT expression increases hepatic folate contents and folate-dependent methionine synthase-mediated homocysteine remethylation. Mol Med 17(5-6): 486-494.

Watt, M. J., G. R. Steinberg, Z. P. Chen, B. E. Kemp and M. A. Febbraio (2006). Fatty acids stimulate AMP-activated protein kinase and enhance fatty acid oxidation in L6 myotubes. $\underline{\mathbf{J}}$ Physiol 574(Pt 1): 139-147.

Westerbacka, J., M. Kolak, T. Kiviluoto, P. Arkkila, J. Siren, A. Hamsten, R. M. Fisher and H. Yki-Jarvinen (2007). Genes involved in fatty acid partitioning and binding, lipolysis, monocyte/macrophage recruitment, and inflammation are overexpressed in the human fatty liver of insulin-resistant subjects. Diabetes 56(11): 2759-2765.

Wieckowska, A. and A. E. Feldstein (2008). Diagnosis of nonalcoholic fatty liver disease: invasive versus noninvasive. Semin Liver Dis 28(4): 386-395.

Wieckowska, A., B. G. Papouchado, Z. Li, R. Lopez, N. N. Zein and A. E. Feldstein (2008). Increased hepatic and circulating interleukin-6 levels in human nonalcoholic steatohepatitis. Am J Gastroenterol 103(6): 1372-1379.

Wilkening, S., F. Stahl and A. Bader (2003). Comparison of primary human hepatocytes and hepatoma cell line Hepg2 with regard to their biotransformation properties. Drug Metab Dispos 31(8): 1035-1042.

Winzell, M. S. and B. Ahren (2004). The high-fat diet-fed mouse: a model for studying mechanisms and treatment of impaired glucose tolerance and type 2 diabetes. Diabetes 53 Suppl 3: S215-219. 
Wong, R. J., M. Aguilar, R. Cheung, R. B. Perumpail, S. A. Harrison, Z. M. Younossi and A. Ahmed (2015). Nonalcoholic steatohepatitis is the second leading etiology of liver disease among adults awaiting liver transplantation in the United States. Gastroenterology 148(3): 547555.

Wong, R. J., R. Cheung and A. Ahmed (2014). Nonalcoholic steatohepatitis is the most rapidly growing indication for liver transplantation in patients with hepatocellular carcinoma in the U.S. Hepatology 59(6): 2188-2195.

Woo, C. W., G. A. Prathapasinghe, Y. L. Siow and K. O (2006). Hyperhomocysteinemia induces liver injury in rat: Protective effect of folic acid supplementation. Biochim Biophys Acta 1762(7): 656-665.

Woo, C. W., Y. L. Siow and K. O (2006). Homocysteine activates cAMP-response element binding protein in HepG2 through cAMP/PKA signaling pathway. Arterioscler Thromb Vasc Biol 26(5): 1043-1050.

Woo, C. W., Y. L. Siow and K. O (2008). Homocysteine induces monocyte chemoattractant protein-1 expression in hepatocytes mediated via activator protein-1 activation. $\mathrm{J}$ Biol Chem 283(3): 1282-1292.

Woo, C. W., Y. L. Siow, G. N. Pierce, P. C. Choy, G. Y. Minuk, D. Mymin and K. O (2005). Hyperhomocysteinemia induces hepatic cholesterol biosynthesis and lipid accumulation via activation of transcription factors. Am J Physiol Endocrinol Metab 288(5): E1002-1010.

Woods, A., S. R. Johnstone, K. Dickerson, F. C. Leiper, L. G. Fryer, D. Neumann, U. Schlattner, T. Wallimann, M. Carlson and D. Carling (2003). LKB1 is the upstream kinase in the AMPactivated protein kinase cascade. Curr Biol 13(22): 2004-2008.

Worm, J., A. F. Kirkin, K. N. Dzhandzhugazyan and P. Guldberg (2001). Methylation-dependent silencing of the reduced folate carrier gene in inherently methotrexate-resistant human breast cancer cells. J Biol Chem 276(43): 39990-40000.

Wortham, M., L. He, M. Gyamfi, B. L. Copple and Y. J. Wan (2008). The transition from fatty liver to NASH associates with SAMe depletion in $\mathrm{db} / \mathrm{db}$ mice fed a methionine choline-deficient diet. Dig Dis Sci 53(10): 2761-2774.

Wree, A., L. Broderick, A. Canbay, H. M. Hoffman and A. E. Feldstein (2013). From NAFLD to NASH to cirrhosis-new insights into disease mechanisms. Nat Rev Gastroenterol Hepatol 10(11): 627-636. 
Wright, A. J., J. R. Dainty and P. M. Finglas (2007). Folic acid metabolism in human subjects revisited: potential implications for proposed mandatory folic acid fortification in the UK. $\underline{\mathrm{Br} J}$ Nutr 98(4): 667-675.

Wu, K., E. A. Platz, W. C. Willett, C. S. Fuchs, J. Selhub, B. A. Rosner, D. J. Hunter and E. Giovannucci (2009). A randomized trial on folic acid supplementation and risk of recurrent colorectal adenoma. Am J Clin Nutr 90(6): 1623-1631.

Wu, N., L. K. Sarna, S. Y. Hwang, Q. Zhu, P. Wang, Y. L. Siow and K. O (2013). Activation of 3-hydroxy-3-methylglutaryl coenzyme A (HMG-CoA) reductase during high fat diet feeding. Biochim Biophys Acta 1832(10): 1560-1568.

Wu, N., L. K. Sarna, Y. L. Siow and K. O (2011). Regulation of hepatic cholesterol biosynthesis by berberine during hyperhomocysteinemia. Am J Physiol Regul Integr Comp Physiol 300(3): R635-643.

Wu, N., Y. L. Siow and K. O (2009). Induction of hepatic cyclooxygenase-2 by hyperhomocysteinemia via nuclear factor-kappaB activation. Am J Physiol Regul Integr Comp Physiol 297(4): R1086-1094.

Xia, M. F., H. Bian, X. P. Zhu, H. M. Yan, X. X. Chang, L. S. Zhang, H. D. Lin, X. Q. Hu and $X$. Gao (2018). Serum folic acid levels are associated with the presence and severity of liver steatosis in Chinese adults. Clin Nutr 37(5): 1752-1758.

Xie, Z., Y. Dong, R. Scholz, D. Neumann and M. H. Zou (2008). Phosphorylation of LKB1 at serine 428 by protein kinase $\mathrm{C}$-zeta is required for metformin-enhanced activation of the AMPactivated protein kinase in endothelial cells. Circulation 117(7): 952-962.

Xu, H., G. T. Barnes, Q. Yang, G. Tan, D. Yang, C. J. Chou, J. Sole, A. Nichols, J. S. Ross, L. A. Tartaglia and H. Chen (2003). Chronic inflammation in fat plays a crucial role in the development of obesity-related insulin resistance. J Clin Invest 112(12): 1821-1830.

Xu, X. J., M. S. Gauthier, D. T. Hess, C. M. Apovian, J. M. Cacicedo, N. Gokce, M. Farb, R. J. Valentine and N. B. Ruderman (2012). Insulin sensitive and resistant obesity in humans: AMPK activity, oxidative stress, and depot-specific changes in gene expression in adipose tissue. $\underline{\mathrm{J} \text { Lipid }}$ Res 53(4): 792-801.

Yang, J., S. Maika, L. Craddock, J. A. King and Z. M. Liu (2008). Chronic activation of AMPactivated protein kinase-alpha1 in liver leads to decreased adiposity in mice. Biochem Biophys Res Commun 370(2): 248-253. 
Yang, S. Q., H. Z. Lin, M. D. Lane, M. Clemens and A. M. Diehl (1997). Obesity increases sensitivity to endotoxin liver injury: implications for the pathogenesis of steatohepatitis. Proc Natl Acad Sci U S A 94(6): 2557-2562.

Yao, Z. M. and D. E. Vance (1990). Reduction in VLDL, but not HDL, in plasma of rats deficient in choline. Biochem Cell Biol 68(2): 552-558.

Yeh, M. M. and E. M. Brunt (2014). Pathological features of fatty liver disease. Gastroenterology 147(4): 754-764.

Yoneda, M., Y. Guo, H. Ono, Y. Nakatsu, J. Zhang, X. Cui, M. Iwashita, S. Kumamoto, Y. Tsuchiya, H. Sakoda, M. Fujishiro, A. Kushiyama, Y. Koketsu, T. Kikuchi, H. Kamata, F. Nishimura and T. Asano (2010). Decreased SIRT1 expression and LKB1 phosphorylation occur with long-term high-fat diet feeding, in addition to AMPK phosphorylation impairment in the early phase. Obes Res Clin Pract 4(3): e163-246.

Younossi, Z., Q. M. Anstee, M. Marietti, T. Hardy, L. Henry, M. Eslam, J. George and E. Bugianesi (2018). Global burden of NAFLD and NASH: trends, predictions, risk factors and prevention. Nat Rev Gastroenterol Hepatol 15(1): 11-20.

Younossi, Z. M., A. B. Koenig, D. Abdelatif, Y. Fazel, L. Henry and M. Wymer (2016). Global epidemiology of nonalcoholic fatty liver disease-Meta-analytic assessment of prevalence, incidence, and outcomes. Hepatology 64(1): 73-84.

Younossi, Z. M., R. Loomba, M. E. Rinella, E. Bugianesi, G. Marchesini, B. A. NeuschwanderTetri, L. Serfaty, F. Negro, S. H. Caldwell, V. Ratziu, K. E. Corey, S. L. Friedman, M. F. Abdelmalek, S. A. Harrison, A. J. Sanyal, J. E. Lavine, P. Mathurin, M. R. Charlton, N. P. Chalasani, Q. M. Anstee, K. V. Kowdley, J. George, Z. D. Goodman and K. Lindor (2018). Current and future therapeutic regimens for nonalcoholic fatty liver disease and nonalcoholic steatohepatitis. Hepatology 68(1): 361-371.

Younossi, Z. M., M. Stepanova, F. Negro, S. Hallaji, Y. Younossi, B. Lam and M. Srishord (2012). Nonalcoholic fatty liver disease in lean individuals in the United States. Medicine (Baltimore) 91(6): 319-327.

Zhang, B. B., G. Zhou and C. Li (2009). AMPK: an emerging drug target for diabetes and the metabolic syndrome. Cell Metab 9(5): 407-416. 
Zhang, S., L. Zheng, D. Dong, L. Xu, L. Yin, Y. Qi, X. Han, Y. Lin, K. Liu and J. Peng (2013). Effects of flavonoids from Rosa laevigata Michx fruit against high-fat diet-induced nonalcoholic fatty liver disease in rats. Food Chem 141(3): 2108-2116.

Zhao, R., N. Diop-Bove, M. Visentin and I. D. Goldman (2011). Mechanisms of membrane transport of folates into cells and across epithelia. Annu Rev Nutr 31: 177-201.

Zhao, R., L. H. Matherly and I. D. Goldman (2009). Membrane transporters and folate homeostasis: intestinal absorption and transport into systemic compartments and tissues. Expert Rev Mol Med 11: e4.

Zhao, R., R. G. Russell, Y. Wang, L. Liu, F. Gao, B. Kneitz, W. Edelmann and I. D. Goldman (2001). Rescue of embryonic lethality in reduced folate carrier-deficient mice by maternal folic acid supplementation reveals early neonatal failure of hematopoietic organs. J Biol Chem 276(13): 10224-10228.

Zhao, Y., B. Su, R. L. Jacobs, B. Kennedy, G. A. Francis, E. Waddington, J. T. Brosnan, J. E. Vance and D. E. Vance (2009). Lack of phosphatidylethanolamine N-methyltransferase alters plasma VLDL phospholipids and attenuates atherosclerosis in mice. Arterioscler Thromb Vasc Biol 29(9): 1349-1355.

Zhou, G., R. Myers, Y. Li, Y. Chen, X. Shen, J. Fenyk-Melody, M. Wu, J. Ventre, T. Doebber, N. Fujii, N. Musi, M. F. Hirshman, L. J. Goodyear and D. E. Moller (2001). Role of AMPactivated protein kinase in mechanism of metformin action. J Clin Invest 108(8): 1167-1174. 


\section{APPENDIX}




\section{Appendix I}

Composition of purified diets: energy, macronutrient and other primary ingredients

\begin{tabular}{|c|c|c|c|c|c|c|}
\hline & \multicolumn{2}{|c|}{$\begin{array}{c}\text { Control } \\
\text { (D12450B) }\end{array}$} & \multicolumn{2}{|c|}{$\begin{array}{l}\text { High-fat diet } \\
\text { (D12492) }\end{array}$} & \multicolumn{2}{|c|}{$\begin{array}{c}\text { High-fat diet } \\
\text { +Folic acid }\end{array}$} \\
\hline & \multicolumn{2}{|c|}{$\%$ kcal } & \multicolumn{2}{|c|}{$\%$ kcal } & \multicolumn{2}{|c|}{$\%$ kcal } \\
\hline Protein & \multicolumn{2}{|c|}{20} & \multicolumn{2}{|c|}{20} & \multicolumn{2}{|c|}{20} \\
\hline Carbohydrate & \multicolumn{2}{|c|}{70} & \multicolumn{2}{|c|}{20} & \multicolumn{2}{|c|}{20} \\
\hline Fat & \multicolumn{2}{|c|}{10} & \multicolumn{2}{|c|}{60} & \multicolumn{2}{|c|}{60} \\
\hline kcal/g & \multicolumn{2}{|c|}{3.85} & \multicolumn{2}{|c|}{5.24} & \multicolumn{2}{|c|}{5.24} \\
\hline & $\mathbf{g}$ & kcal & $\mathbf{g}$ & kcal & $\mathbf{g}$ & kcal \\
\hline \multicolumn{7}{|l|}{ Purified Ingredients } \\
\hline Casein, 80 Mesh & 200 & 800 & 200 & 800 & 200 & 800 \\
\hline L-Cystine & 3 & 12 & 3 & 12 & 3 & 12 \\
\hline Corn Starch & 315 & 1260 & 0 & 0 & 0 & 0 \\
\hline Maltodextrin 10 & 35 & 140 & 125 & 500 & 125 & 500 \\
\hline Sucrose & 350 & 1400 & 68.8 & 275.2 & 68.8 & 275.2 \\
\hline Cellulose, BW200 & 50 & 0 & 50 & 0 & 50 & 0 \\
\hline Soybean Oil & 25 & 225 & 25 & 225 & 25 & 225 \\
\hline Lard & 20 & 180 & 245 & 2205 & 245 & 2205 \\
\hline Mineral Mix, S10026 & 10 & 0 & 10 & 0 & 10 & 0 \\
\hline DiCalcium Phosphate & 13 & 0 & 13 & 0 & 13 & 0 \\
\hline Calcium Carbonate & 5.5 & 0 & 5.5 & 0 & 5.5 & 0 \\
\hline Potassium Citrate, $1 \mathrm{H} 2 \mathrm{O}$ & 16.5 & 0 & 16.5 & 0 & 16.5 & 0 \\
\hline Vitamin Mix, V10001 & 10 & 40 & 10 & 40 & 10 & 40 \\
\hline Choline Bitartrate & 2 & 0 & 2 & 0 & 2 & 0 \\
\hline Folic acid & 0 & 0 & 0 & 0 & 0.018 & 0 \\
\hline FD\&C Yellow Dye \#5 & 0.05 & 0 & 0 & 0 & 0 & 0 \\
\hline FD\&C Red Dye \#40 & 0 & 0 & 0 & 0 & 0.025 & 0 \\
\hline FD\&C Blue Dye \#1 & 0 & 0 & 0.05 & 0 & 0.025 & 0 \\
\hline Total & 1055.05 & 4057 & 773.85 & 4057 & 773.87 & 4057 \\
\hline Folic acid, mg per kg diet & \multicolumn{2}{|c|}{2.0} & \multicolumn{2}{|c|}{2.6} & \multicolumn{2}{|c|}{26} \\
\hline
\end{tabular}

Based on product data sheets obtained from Research Diets, Inc.

* Diets are formulated on the basis of their to nutrient calorie ratios in order to ensure that the diets are consumed for calories. Therefore, discrepancies in intake relate only to carbohydrate and fat contents of the diet and not to other primary ingredients, including protein, micronutrients and fiber. 


\section{Appendix II}

Composition of purified diets: fatty acid profile

\begin{tabular}{|c|c|c|c|c|c|c|}
\hline \multirow[t]{2}{*}{$\begin{array}{c}\text { Fatty acid } \\
\text { composition }\end{array}$} & \multicolumn{2}{|c|}{$\begin{array}{c}\text { Control } \\
\text { (D12450B) }\end{array}$} & \multicolumn{2}{|c|}{$\begin{array}{l}\text { High-fat diet } \\
\text { (D12492) }\end{array}$} & \multicolumn{2}{|c|}{ High-fat diet +Folic acid } \\
\hline & $\mathrm{g} / \mathbf{1 0 5 5 . 0 5 g}$ & \% of Lipid & $\mathrm{g} / 773.85 \mathrm{~g}$ & \% of Lipid & $\mathrm{g} / 773.85 \mathrm{~g}$ & $\%$ of Lipid \\
\hline Saturated & 9.9 & 22.7 & 81.5 & 32.0 & 81.5 & 32.0 \\
\hline Monounsaturated & 13.0 & 29.9 & 91.5 & 35.9 & 91.5 & 35.9 \\
\hline Polyunsaturated & 20.7 & 47.4 & 81.5 & 32.0 & 81.5 & 32.0 \\
\hline Total & 43.7 & & 254.5 & & 254.5 & \\
\hline Fatty acid profile & \multicolumn{2}{|c|}{ g/4057kcal } & \multicolumn{2}{|c|}{ g/4057kcal } & \multicolumn{2}{|c|}{ g/4057kcal } \\
\hline C10, Capric & \multicolumn{2}{|c|}{0.0} & \multicolumn{2}{|c|}{0.1} & \multicolumn{2}{|c|}{0.1} \\
\hline C12, Lauric & \multicolumn{2}{|c|}{0.0} & \multicolumn{2}{|c|}{0.2} & \multicolumn{2}{|c|}{0.2} \\
\hline C14, Myristic & \multirow{2}{*}{\multicolumn{2}{|c|}{0.2}} & \multirow{2}{*}{\multicolumn{2}{|c|}{2.8}} & \multirow{2}{*}{\multicolumn{2}{|c|}{2.8}} \\
\hline $\mathrm{C} 15$ & & & & & & \\
\hline C16, Palmitic & \multicolumn{2}{|c|}{6.5} & \multicolumn{2}{|c|}{49.9} & \multicolumn{2}{|c|}{49.9} \\
\hline C16:1, Palmitoleic & \multicolumn{2}{|c|}{0.3} & \multicolumn{2}{|c|}{3.4} & \multicolumn{2}{|c|}{3.4} \\
\hline $\mathrm{C} 17$ & \multicolumn{2}{|c|}{0.1} & \multicolumn{2}{|c|}{0.9} & \multicolumn{2}{|c|}{0.9} \\
\hline C18, Stearic & \multicolumn{2}{|c|}{3.1} & \multicolumn{2}{|c|}{26.9} & \multicolumn{2}{|c|}{26.9} \\
\hline C18:1, Oleic & \multirow{2}{*}{\multicolumn{2}{|c|}{12.6}} & \multirow{2}{*}{\multicolumn{2}{|c|}{86.6}} & \multicolumn{2}{|c|}{86.6} \\
\hline C18:2, Linoleic & & & & & & \\
\hline C18:3, Linolenic & \multicolumn{2}{|c|}{$\frac{18.3}{2.2}$} & \multicolumn{2}{|c|}{$\begin{array}{l}73.1 \\
5.2\end{array}$} & & \\
\hline C20, Arachidic & & 0 & & 4 & & \\
\hline $\mathrm{C} 20: 1$ & & 1 & & 5 & & \\
\hline $\mathrm{C} 20: 2$ & & 2 & & 0 & & \\
\hline $\mathrm{C} 20: 3$ & & 0 & & 3 & & \\
\hline C20:4, Arachidonic & & 1 & & 7 & & \\
\hline $\begin{array}{c}\text { C22:5, } \\
\text { Docasasapentaenoic }\end{array}$ & & 0 & & 2 & & \\
\hline Total $(\mathrm{g})$ & 4. & .7 & & 4.7 & & \\
\hline
\end{tabular}

Fatty acid profile was calculated based on assays of fats and oils performed in the $4^{\text {th }}$ quarter of 2011

Based on product data sheets obtained from Research Diets, Inc. 


\section{Appendix III}

Composition of purified diets: typical amino acid profile

\begin{tabular}{|c|c|c|c|c|c|c|}
\hline Amino Acid & \multicolumn{2}{|c|}{$\begin{array}{c}\text { Control } \\
\text { (D12450B) }\end{array}$} & \multicolumn{2}{c|}{$\begin{array}{c}\text { High-fat diet } \\
\text { (D12492) }\end{array}$} & \multicolumn{2}{c|}{$\begin{array}{c}\text { High-fat diet + } \\
\text { Folic acid }\end{array}$} \\
\hline Essential amino acids & $\begin{array}{c}\text { g/4057 } \\
\text { kcal }\end{array}$ & $\begin{array}{c}\text { \% diet } \\
\text { weight }\end{array}$ & $\begin{array}{c}\text { g/4057 } \\
\text { kcal }\end{array}$ & $\begin{array}{c}\text { \% diet } \\
\text { weight }\end{array}$ & $\begin{array}{c}\text { g/4057 } \\
\text { kcal }\end{array}$ & $\begin{array}{c}\text { \% diet } \\
\text { weight }\end{array}$ \\
\hline Isoleucine & 7.6 & 0.72 & 7.6 & 0.98 & 7.6 & 0.98 \\
\hline Leucine & 15.8 & 1.50 & 15.8 & 2.04 & 15.8 & 2.04 \\
\hline Lysine & 13.2 & 1.25 & 13.2 & 1.71 & 13.2 & 1.71 \\
\hline Methionine & 5.1 & 0.48 & 5.1 & 0.66 & 5.1 & 0.66 \\
\hline Phenylalanine & 8.4 & 0.80 & 8.4 & 1.09 & 8.4 & 1.09 \\
\hline Threonine & 7.2 & 0.68 & 7.2 & 0.93 & 7.2 & 0.93 \\
\hline Tryptophan & 2.1 & 0.20 & 2.1 & 0.27 & 2.1 & 0.27 \\
\hline Valine & 9.3 & 0.88 & 9.3 & 1.20 & 9.3 & 1.20 \\
\hline Non-essential amino acids & & & & & & \\
\hline Histidine & 4.6 & 0.44 & 4.6 & 0.59 & 4.6 & 0.59 \\
\hline Alanine & 5.1 & 0.48 & 5.1 & 0.66 & 5.1 & 0.66 \\
\hline Arginine & 6.0 & 0.57 & 6.0 & 0.78 & 6.0 & 0.78 \\
\hline Aspartic acid & 12.1 & 1.15 & 12.1 & 1.56 & 12.1 & 1.56 \\
\hline Cysteine/cystine & 4.2 & 0.40 & 4.2 & 0.54 & 4.2 & 0.54 \\
\hline Glutamic acid & 38.2 & 3.62 & 38.2 & 4.94 & 38.2 & 4.94 \\
\hline Glycine & 3.0 & 0.28 & 3.0 & 0.39 & 3.0 & 0.39 \\
\hline Proline & 17.8 & 1.69 & 17.8 & 2.30 & 17.8 & 2.30 \\
\hline Serine & 10.0 & 0.95 & 10.0 & 1.29 & 10.0 & 1.29 \\
\hline Tyrosine & 9.2 & 0.87 & 9.2 & 1.19 & 9.2 & 1.19 \\
\hline \multicolumn{7}{|l|}{} \\
\hline Total essential amino acids & $\mathbf{7 9 . 3}$ & 7.52 & $\mathbf{7 9 . 3}$ & 10.25 & $\mathbf{7 9 . 3}$ & 10.25 \\
\hline $\begin{array}{c}\text { Total non-essential } \\
\text { amino acids }\end{array}$ & $\mathbf{9 9 . 6}$ & 9.44 & $\mathbf{9 9 . 6}$ & 12.87 & $\mathbf{9 9 . 6}$ & 12.87 \\
\hline Total amino acids & $\mathbf{1 7 8 . 9}$ & 16.96 & $\mathbf{1 7 8 . 9}$ & 23.12 & $\mathbf{1 7 8 . 9}$ & 23.12 \\
\hline
\end{tabular}

Amino acid profile per $200 \mathrm{~g}$ casein and $3 \mathrm{~g}$ of L-cystine

Based on product data sheets obtained from Research Diets, Inc. 